\title{
Ultrahigh Specific Impulse Nuclear Thermal Propulsion
}

\author{
DOE Award Number: DE-FG07-05ID14699
}

\author{
Final Report \\ Period covered: Jul 01, 2005 - Jun 30, 2008 \\ Anne Charmeau \\ Brandon Cunningham \\ Samim Anghaie, PI \\ Department of Nuclear \& Radiological Engineering \\ 202 NSC \\ University of Florida \\ Gainesville, FL 32611-8300
}

February 9, 2009 


\section{PREFACE}

Research on nuclear thermal propulsion systems (NTP) have been in forefront of the space nuclear power and propulsion due to their design simplicity and their promise for providing very high thrust at reasonably high specific impulse. During NERVA-ROVER program in late 1950's till early 1970's, the United Sates developed and ground tested about 18 NTP systems without ever deploying them into space. The NERVA-ROVER program included development and testing of NTP systems with very high thrust $\left(\sim 250,000 \mathrm{lb}_{\mathrm{f}}\right)$ and relatively high specific impulse $(\sim 850 \mathrm{~s})$. High thrust to weight ratio in NTP systems is an indicator of high acceleration that could be achieved with these systems. The specific impulse in the lowest mass propellant, hydrogen, is a function of square root of absolute temperature in the NTP thrust chamber. Therefore, optimizing design performance of NTP systems would require achieving the highest possible hydrogen temperature at reasonably high thrust to weight ratio. High hydrogen exit temperature produces high specific impulse that is a direct measure of propellant usage efficiency.

After the cancellation of the US NERVA-ROVER program in early 1970's, the Former Soviet Union continued with the US effort and made improvement in fuel design that enabled design and ground testing of an NTP systems (1984) at temperatures and specific impulses well above what had been achieved during the NERVA-ROVER program (hydrogen exit temperature $\sim 3,000 \mathrm{~K}$ and specific impulse $\sim 1,000 \mathrm{~s}$ ). The renewed interest in the space nuclear power and propulsion is the main motivation for the proposed research that is intended to develop reactor design and NTP systems for achieving the highest possible specific impulse

Research conducted in support of the development of a new nuclear powered thermal rocket propulsion system is proposed to provide variable thrust in the range of 1,000 to 10,000 $\mathrm{lb}_{\mathrm{f}}$ of thrust at 1000-1500 seconds of ideal specific impulse (Isp) by heating relatively lowpressure hydrogen propellant to average temperatures ranging from $3000 \mathrm{~K}$ to $3250 \mathrm{~K}$. The reactor for the proposed NTR concept is fueled with a uranium tetra-carbide compound that is optimized for resisting atomic and molecular hydrogen at temperatures above $3000 \mathrm{~K}$. Uranium tetra- and tri-carbide fuels feature the highest corrosion resistance properties at elevated temperatures in hydrogen environment. The congruent melting of tetra- and tri-carbides is not overly sensitive to carbon content, as it's the case for uranium bi-carbides such as uranium- 
zirconium and uranium-niobium carbides. This is a key property of these fuels that would enable their applications for operation at temperatures above $3000 \mathrm{~K}$. The fuel candidate for this concept is the solid solution of uranium-zirconium-niobium-titanium carbide, $(\mathrm{U}, \mathrm{Zr}, \mathrm{Nb}, \mathrm{Ti}) \mathrm{C}$. Compare to uranium tri-carbides that have previously been considered for application in NTP systems, the proposed uranium tetra-carbide features higher stability in dissociated hydrogen and more favorable neutronic characteristics than tri-carbides with tantalum or hafnium component. The fuel form and reactor core are designed for achieving very high surface to volume ratio that would allow using hydrogen propellant at pressures as low as .1 bar. Under a specific operational temperature and pressure regime, the dissociated hydrogen could expand through the nozzle and produce specific impulses far above what has ever been achieved or proposed for any thermal propulsion system.

The report includes results of research in two separate parts. The first part (Part I) provides a detailed description of model development and application for the analysis of the ultrahigh impulse nuclear thermal propulsion system. The second part of the report summarizes results of research on the testing and characterization of three monocarbides and four uranium tricarbides that are considered for application in the reactor core and nozzle of the thermal propulsion system. 


\section{$\underline{\text { Part I }}$}

\section{Hybrid Fine-Coarse Mesh Computational Fluid Dynamics and Heat Transfer Model}




\section{PART I}

\section{TABLE OF CONTENTS}

page

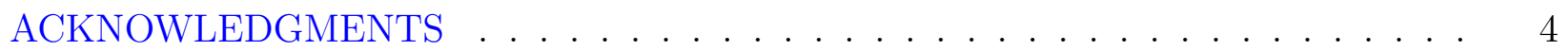

LIST OF TABLES . . . . . . . . . . . . . . . . . . . . . 9

LIST OF FIGURES . . . . . . . . . . . . . . . . . . . . . 10

LIST OF ACRONYMS . . . . . . . . . . . . . . . . . . . 13

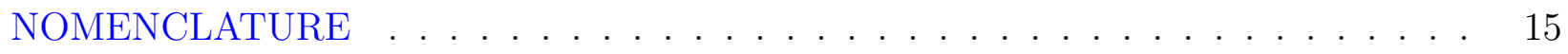

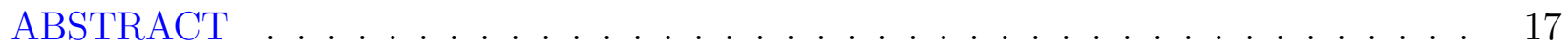

CHAPTER

1 INTRODUCTION: ANSWERING THE NEEDS OF THE FUTURE IN THE NUCLEAR INDUSTRY AND SYSTEM MODELING . . . . . . . . . . . . . 21

1.1 The Nuclear Power Scene of the 21st Century . . . . . . . . . . . . . . 21

1.2 Challenges in Thermal-Hydraulics . . . . . . . . . . . . . . . . . . . 22

1.3 Issues Faced with the Current Computational Tools for Thermal-Hydraulics 23

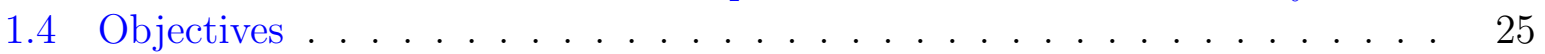

1.5 The Different Approaches . . . . . . . . . . . . . . . . . . 26

1.6 Overview of our Research . . . . . . . . . . . . . . . . 27

2 APPLICATION FUNDAMENTALS . . . . . . . . . . . . . . . . 29

2.1 Space Propulsion and Nuclear Thermal Propulsion . . . . . . . . . . . . 29

2.2 The Low-Pressure Thrust Chamber . . . . . . . . . . . . . . . . . . . . 31

2.3 Computational Fluid Dynamics: An Overview . . . . . . . . . . . . . . . 32

3 A SURVEY ON STATE-OF-THE-ART COMPUTATIONAL FLUID DYNAMICS DEVELOPMENTS AND APPLICATIONS . . . . . . . . . . . . . . 35

3.1 System Coupling . . . . . . . . . . . . . . . . . . . . 40

3.2 Numerical Techniques for CFD Solvers . . . . . . . . . . . . . . . . . 42

3.2.1 Considerations on Compressible Flows . . . . . . . . . . . . . . . . . 42

3.2.2 Discussion on the MacCormack Methods . . . . . . . . . . . . . . . 44

3.2.3 Considerations on Turbulence . . . . . . . . . . . . . . . . . . . 47

3.2.4 Discussion on the Spalart-Allmaras Model . . . . . . . . . . . . 50

4 MATERIAL PROPERTIES . . . . . . . . . . . . . . . . . . . 52

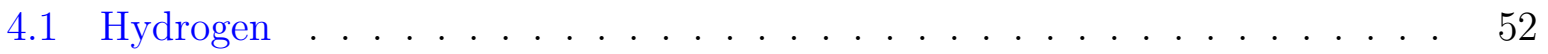

4.1.1 Note on Dissociation . . . . . . . . . . . . . . . 53

4.1.2 Thermo-Physical Properties . . . . . . . . . . . . . . 54

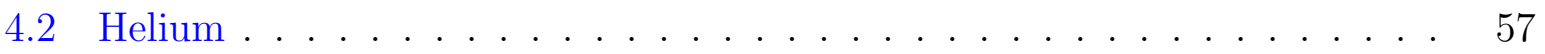


4.3 Carbon Dioxide . . . . . . . . . . . . . . . . . . . . 58

5 METHODS FOR THE CFD SOLVER DEVELOPMENT _ . . . . . . . . . . 73

5.1 CFD Solver Development . . . . . . . . . . . . . . . . . 73

5.1 .1 Requirements . . . . . . . . . . . . . . . . . . . . 73

5.1.2 Governing Equations for Compressible Flow . . . . . . . . . . 74

5.1 .3 Need for the Equation of State . . . . . . . . . . . . . . 76

5.1.4 Reynolds-Averaged Navier-Stokes Equations . . . . . . . . . . . 77

5.1.5 Closure Problem: the Need for Turbulence Model . . . . . . . . . 78

5.1.5.1 Turbulence models: classification and overview . . . . . . 79

5.1.5.2 k- $\epsilon$ model . . . . . . . . . . . . . . . . . . . . 80

5.1.5.3 The Spalart-Allmaras model . . . . . . . . . . . . . . 81

5.1 .6 Space Discretization . . . . . . . . . . . . . . . . . . . . 83

5.1.7 Time and Space Discretization: TVD MacCormack Scheme . . . . . 86

5.1 .8 Time Stepping . . . . . . . . . . . . . . . . . . . . 88

5.1 .9 Boundary Conditions . . . . . . . . . . . . . . . 88

5.1.10 Limiters and Total Variation Diminishing Condition . . . . . . . . 91

5.1 .10 .1 Full MacCormack scheme . . . . . . . . . . . . . . 93

5.1 .10 .2 Modified Causon limiter . . . . . . . . . . . . . . 94

5.2 Remark on the Practical Implementation of the Real Gas Properties - Algorithm 95

5.3 Validation Cases: The Riemann Shock Tube . . . . . . . . . . . . . . . 97

5.3 .1 Presentation of the Test Case . . . . . . . . . . . . . . . 97

5.3 .2 Numerical Method . . . . . . . . . . . . . . . . . . . . . . 98

5.3 .3 Results . . . . . . . . . . . . . . . . . . . . . . . . . 99

5.3.3.1 On the influence of the gas . . . . . . . . . . . . 99

6 CFD STUDY OF THE COOLANT CHANNELS OF A PRISMATIC VHTR . . 109

6.1 Grid Generation . . . . . . . . . . . . . . . . . . . . . . . . . 111

6.2 Application of the TVD MacCormack Scheme - Derivation of the Viscous

Term . . . . . . . . . . . . . . . . . . . . 111

6.3 Simulation Results . . . . . . . . . . . . . . . . . . . . . . 113

6.3.1 Validation of the Model . . . . . . . . . . . . . . . . . . . 113

6.3.1.1 Boundaries and characteristics . . . . . . . . . . . 113

6.3.1.2 Results and simulation comparisons . . . . . . . . . . 114

6.3.2 On the influence of the real properties simulation . . . . . . . 114

6.3.3 Modeling of the VHTR Coolant Channel . . . . . . . . . . . . 115

6.4 Conclusion on the Helium Channel Study with Real Property Fine CFD

Solver . . . . . . . . . . . . . . . . . . . . . 115

7 CFD STUDY OF THE LOW-PRESSURE THRUST CHAMBER . . . . . . . . 125

7.1 Presentation of the Low-Pressure Thrust Chamber . . . . . . . . . . . 125

7.2 Quasi-one-dimension Isentropic Analysis of the Low Thrust Chamber . . . 126

7.3 Two-Dimensional Contour Profile of the Thrust Chamber Walls . . . . . . 128

7.4 Grid Generation . . . . . . . . . . . . . . . . . . 130 
7.5 Low-Pressure Thrust Chamber Analysis using FLUENT and Frozen Chemistry 130

$7.5 .1 \quad$ FLUENT . . . . . . . . . . . . . . . . . . . . . . . . . 130

7.5 .2 Numerical Procedure . . . . . . . . . . . . . . . . . . . . . . 130

7.5.3 Analysis results . . . . . . . . . . . . . . . . . . . . . . 132

7.5.3.1 Investigation of the nozzle representation . . . . . . . . . 132

7.5.3.2 Which governing equation to use? . . . . . . . . . . . 133

7.5.3.3 Investigation of the turbulence model . . . . . . . . . . 134

7.5.3.4 Sensitivity study . . . . . . . . . . . . . . . . . 134

7.5.4 Design Parameters Sensitivity Study . . . . . . . . . . . . . . 135

7.5.4.1 Influence of the thermal formulation . . . . . . . . 137

7.5.4.2 Influence of the nozzle slope at the exit of the system . . . 137

7.6 Our in-house solver . . . . . . . . . . . . . . . . . . . . . . . . 137

8 COUPLING OF COARSE AND FINE MESH SOLVERS . . . . . . . . . . . . 147

8.1 NuRok: a 1-D System Code for Simulation of NTP Thermal-Hydraulics . . 147

8.1.1 Description of NuRok Core Subroutine and One-Dimensional Correlations149

8.1 .2 NuRok Upgrading . . . . . . . . . . . . . . . . . . . . . . . . 152

8.1.2.1 Update to FORTRAN $90 \ldots \ldots \ldots \ldots \ldots$

8.1.2.2 Levels of thrust . . . . . . . . . . . . . . . . 153

8.1.2.3 Foam fuel . . . . . . . . . . . . . . . . . . . . . 154

8.1.2.4 Hydrogen dissociation . . . . . . . . . . . . . . . 154

8.1.2.5 Carbon dioxide . . . . . . . . . . . . . . . 154

8.1.3 Recommendations for NuRok Users . . . . . . . . . . . . . . 155

8.1.3.1 Mass Flow Rate . . . . . . . . . . . . . . . . . . 155

8.1.3.2 Pump Outlet Pressure . . . . . . . . . . . . . . . . . . 155

8.1.3.3 Core Power . . . . . . . . . . . . . . . . 155

8.2 Themis: A Coupled Coarse-Fine Mesh Simulation Tool for Simulation of

NTP Thermal-Hydraulics . . . . . . . . . . . . . . . . . . 156

8.2.1 Treatment of the Interfaces . . . . . . . . . . . . . . . 156

8.2.1.1 One-dimension to two-dimension axisymmetric . . . . . . 157

8.2.1.2 Two-dimension axisymmetric to one-dimension . . . . . 157

8.2.2 Themis Results in the Case of NERVA Derivative Systems . . . . 158

8.2.3 Conclusion on the Coupled fine-coarse mesh solver . . . . . . . . 160

9 CONCLUSIONS AND RECOMMENDATIONS . . . . . . . . . . . . . . 171

APPENDIX

A PROGRAM FOR COMPILATION OF THE EXACT RIEMANN PROBLEM SOLUTION . . . . . . . . . . . . . . . . . . . . . 175

B FLUENT PRELIMINARY ANALYZES . . . . . . . . . . . . . . . . . . . . . 182

B.1 Low-Pressure Thrust Chamber: Design Parameter Analysis . . . . . . . . . 182

B.2 Definitions . . . . . . . . . . . . . . . . . . . . . 183 
REFERENCES . . . . . . . . . . . . . . . . . . . . . . . 184

BIOGRAPHICAL SKETCH . . . . . . . . . . . . . . . . . . . . . . . . 192 


\section{LIST OF TABLES}

Table

1-1 Characteristics and operating conditions of different nuclear systems [1] . . . . 28

4-1 Error on the density formulation for $\mathrm{P}=0.1$ bar . . . . . . . . . . . . . . 62

4-2 Error on the density formulation for $\mathrm{P}=1$ bar . . . . . . . . . . . . . 63

4-3 Error on the density formulation for $\mathrm{P}=10$ bar . . . . . . . . . . . . . . . . . . 64

4-4 Error on the density formulation for $\mathrm{P}=100$ bar . . . . . . . . . . . . . . 65

4-5 Error on the density formulation for $\mathrm{P}=150$ bar . . . . . . . . . . . . . . . 66

4-6 VHTR operating conditions and features . . . . . . . . . . . . . . 67

4-7 Comparison between experimental data and ideal-gas law for helium density . . 72

7-1 Hydrogen thermal properties (heat capacity,specific heat ratio and thermal conductivity) for diverse sets of operating conditions . . . . . . . . . . . . . 126

7-2 CPU time (s) and number of iteration to reach convergence of the steady-state for the four different grids . . . . . . . . . . . . . . . . . . . . . . . . . 139

7-3 Test cases for different design parameters . . . . . . . . . . . . . . . . . . . . . 141

8-1 Fanning friction factor correlations and their range of validity [2] . . . . . . . 161

8-2 Nusselt number correlation for turbulent gas flow in a pipe [3] . . . . . . . . . . 161

8-3 Pressure drop over a flow area contraction. Comparison between FLUENT simulation and 1-D correlation results. . . . . . . . . . . . . . . . . 161

8-4 Properties at diverse locations of the square lattice honeycomb system for thrust chamber inlet temperature and pressure of $2,800 \mathrm{~K}$ and $500 \mathrm{psi}(=3.45 \mathrm{MPa})$, respectively. . . . . . . . . . . . . . . . . . . . . 163

8-5 Pressure drop across the SLHC core for different densities of foam fuel. . . . . . 163

8-6 Comparison of the results obtained with NuRok and Themis . . . . . . . . . . . 164 


\section{LIST OF FIGURES}

Figure $\quad \underline{\text { page }}$

2-1 The serial cooling system (NERVA type) . . . . . . . . . . . . . . 34

4-1 Level of hydrogen molecule dissociation as a function of temperature . . . . . . 61

4-2 Hydrogen heat capacity vs. temperature at low and high pressure (0.1 and 150

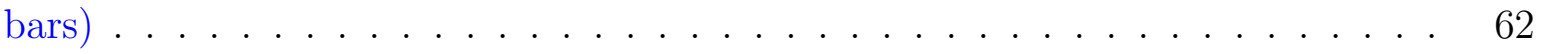

4-3 Hydrogen density at 0.1 bar and high temperature . . . . . . . . . . . . 63

4-4 Hydrogen heat capacity vs. temperature . . . . . . . . . . . . . . . . . . 64

4-5 Hydrogen viscosity vs. temperature . . . . . . . . . . . . . . . . . 65

4-6 Helium viscosity vs. pressure for different temperatures . . . . . . . . . . . . 66

4-7 Helium thermal conductivity vs. pressure for different temperatures . . . . . . . 67

4-8 Carbon dioxide density vs. pressure for different temperatures . . . . . . . . . . 68

4-9 Carbon dioxide heat capacity vs. temperature for different pressures . . . . . . . 69

4-10 Carbon dioxide viscosity vs. temperature for different pressures . . . . . . . . 70

4-11 Carbon dioxide thermal conductivity vs. pressure for different temperatures . 71

5-1 Sketch of the axisymmetric representation of the 3-D channel . . . . . . . . . 101

5-2 Location of the numerical nodes for a $5 \times 5$ domain . . . . . . . . . . . . . . . 102

5-3 Elementary volume (blue area) for integration in the axisymmetric configuration 103

5-4 Details of the notations of the axisymmetric cell $(i, j) \ldots \ldots$. . . . . . . . 104

5-5 Nomenclature for the surface vectors . . . . . . . . . . . . . . . . 105

5-6 Algorithm which shows how the real properties are implemented and computed 106

5-7 The shock tube experiment . . . . . . . . . . . . . . . . 107

5-8 Shock tube profile at $\mathrm{t}=6.1 \mathrm{~ms}$ in case $5-62$. The blue curves are the theoretical profiles and the green curves the profiles computed with our Euler solver . . . . 107

5-9 Properties profile at $\mathrm{t}=6.1 \mathrm{~ms}$ in case $5-62$ for air, helium, carbon dioxide and uranium tetrafluoride. . . . . . . . . . . . . . . . . . 108

6-1 VHTR coolant channel . . . . . . . . . . . . . . . . . . . 116

6-2 Dual cell for the approximation of the derivatives at the cell interface $i+\frac{1}{2}, j \ldots 117$ 
6-3 Helium channel mesh: 100x1000 quadrilateral grid zoomed at the inlet of the channel . . . . . . . . . . . . . . . . . . . . . . . . . . . . 117

6-4 Static pressure (Pa) profile on the axis of the test-section. Comparison between FLUENT simulations and our solver. . . . . . . . . . . . . . . . . . . . . 118

6-5 Velocity $(\mathrm{m} / \mathrm{s})$ profile on the axis of the test-section. Comparison between FLUENT simulations and our solver. . . . . . . . . . . . . . . . . . . . . . . . . . . 119

6-6 Temperature (K) profile on the axis of the test-section. Comparison between FLUENT simulations and our solver. . . . . . . . . . . . . . . . . . . 120

6-7 Static pressure (Pa) profile on the axis of the test-section. Comparison between constant and real property formulations. . . . . . . . . . . . . . . . . . 121

6-8 Velocity $(\mathrm{m} / \mathrm{s})$ profile on the axis of the test-section. Comparison between constant and real property formulations. . . . . . . . . . . . . . . . . . 122

6-9 Temperature (K) profile on the axis of the test-section.Comparison between constant and real property formulations. . . . . . . . . . . . . . . . . . . 123

6-10 Property profiles across the helium cooling channel. . . . . . . . . . . . . . . . . 124

7-1 Thrust chamber and its principal characteristics . . . . . . . . . . . . . 139

$7-2 \quad$ Coarser grid . . . . . . . . . . . . . . . . . . . . . . 140

7-3 Comparison of the Mach number profile for the axisymmetric and 2-D models . 140

7-4 Mach number profiles for an inviscid and viscous flow of hydrogen . . . . . . . . 142

7-5 Mach number profiles obtained for different turbulence models . . . . . . . . . . 143

7-6 Relative difference in the maximum Mach number between a given grid and the finest grid . . . . . . . . . . . . . . . . . . . . . . . . . . . . 144

7-7 Influence of the design parameters on the maximum velocity on the exhaust plane 145

7-8 Velocity magnitude profile on the exhaust plane of the thrust chamber . . . . 146

8-1 The different NTP systems modeled with NuRok: NVTR, NERVA derivative and $\mathrm{P} \& \mathrm{~W}$ XNR $2000 \mathrm{I} \& \mathrm{II} \ldots \ldots$

8-2 3-D grid of the contraction test section for study with FLUENT. . . . . . . . . . 163

8-3 Static pressure profile along the axis of the contraction test section . . . . . . 164

8-4 Identification and location of portions of the NTP system which would benefit from a more detailed solver . . . . . . . . . . . . . . . . 165

8-5 Structure of the code coupling when the CFD is used to simulate the hot channel 166 
8-6 Extrapolation of the temperature nodes from the axisymmetric geometry to a single value. . . . . . . . . . . . . . . . . . . . . . 167

8-7 Heat profile on the channel walls . . . . . . . . . . . . . . . 168

8-8 Coolant channel pressure profiles calculated with NuRok and Themis . . . . . . 169

8-9 Coolant channel temperature profiles calculated with NuRok and Themis (on the axis and the wall $\ldots \ldots \ldots \ldots \ldots \ldots$ 


\section{LIST OF ACRONYMS}

$\begin{array}{ll}\text { AMG } & \text { Algebraic multigrid } \\ \text { BWR } & \text { Boiling water reactor } \\ \text { CFD } & \text { Computational Fluid Dynamics } \\ \text { DNS } & \text { Direct numerical simulation } \\ \text { FDM } & \text { Finite difference method } \\ \text { FEM } & \text { Finite element method } \\ \text { FVM } & \text { Finite volume method } \\ \text { FOU } & \text { First order upwind } \\ \text { GFR } & \text { Gas-cooled fast reactor } \\ \text { HTGCR } & \text { High temperature gas-core reactor } \\ \text { INL } & \text { Idaho National Laboratory } \\ \text { INSPI } & \text { Innovative Nuclear Space Power and Propulsion Institute } \\ \text { ISP } & \text { Specific impulse } \\ \text { LES } & \text { Large eddy simulation } \\ \text { LWR } & \text { Light water reactor } \\ \text { MUSCL } & \text { Monotone upstream-centered schemes for conservation laws } \\ \text { NASA } & \text { National Aeronautics Space Agency } \\ \text { NEP } & \text { Nuclear electrical propulsion } \\ \text { NTP } & \text { Nuclear thermal propulsion } \\ \text { PDE } & \text { Partial differential equation } \\ \text { P\&W } & \text { Pratt and Whitney } \\ \text { PWR } & \text { Pressurized water reactor } \\ \text { QUICK } & \text { Quadratic upwind differencing } \\ \text { RANS } & \text { Reynolds averaged Navier-Stokes } \\ \text { RNG } & \text { Renormalized group theory } \\ & \end{array}$


RSM Reynolds stress model

SLHC Square lattice honeycomb

SOU Second order upwind

VHTR Very high temperature gas reactor 


\section{LIST OF NOMENCLATURE}

English Symbols

\begin{tabular}{|c|c|}
\hline$a$ & speed of sound (m/s) \\
\hline$A$ & flow cross-sectional area $\mathrm{m}^{2}$ \\
\hline$g$ & gravitational constant \\
\hline$U$ & vector of conservative variables \\
\hline$V$ & vector of primitive variables \\
\hline$R$ & universal gas constant \\
\hline$T$ & temperature $(\mathrm{K})$ \\
\hline$p$ & pressure $(\mathrm{Pa})$ \\
\hline$(u, v)$ & velocity components in cartesian coordinates \\
\hline$\left(u_{r}, u_{z}\right)$ & velocity components in polar coordinates \\
\hline$k$ & thermal conductivity (W/K.s) \\
\hline$M$ & Mach number \\
\hline$M$ & molecular weight \\
\hline$k$ & turbulent kinetic energy \\
\hline$\vec{V}$ & velocity vector \\
\hline$c_{P}$ & heat capacity \\
\hline$Q$ & Heat source \\
\hline$E$ & total energy per unit volume \\
\hline$t$ & time(s) \\
\hline$f$ & body forces \\
\hline$r$ & radius $(\mathrm{cm})$ \\
\hline$r *$ & throat radius $(\mathrm{cm})$ \\
\hline$n$ & number of moles (mol) \\
\hline$R e$ & Reynolds number \\
\hline$P e$ & Peclet number \\
\hline
\end{tabular}




$\begin{array}{ll}D & \text { diameter }(\mathrm{m}) \\ d_{H} & \text { hydraulic diameter }(\mathrm{cm}) \\ (x, y, z) & \text { position in cartesian coordinates } \\ (r, \theta, z) & \text { position in polar coordinates } \\ R_{c} & \text { throat radius of curvature } \\ y_{L}^{\prime} & \text { nozzle exit slope } \\ L & \text { contoured nozzle length } \\ T_{w} & \text { wall temperature }(\mathrm{K})\end{array}$

Greek Symbols

$$
\begin{array}{ll}
\gamma & \text { specific heat ratio } \\
\rho & \text { density }\left(\mathrm{kg} / \mathrm{m}^{3}\right) \\
\epsilon & \text { turbulent dissipation } \\
\mu & \text { viscosity (Pa.s) } \\
\mu_{t} & \text { turbulent viscosity (Pa.s) } \\
\tau & \text { shear stress tensor } \\
\Pi & \text { total shear stress tensor } \\
\delta x & \text { characteristic length (m) } \\
\Gamma & \text { diffusion coefficient } \\
\theta_{a} & \text { subsonic approach angle } \\
\theta & \text { nozzle inflection angle }
\end{array}
$$




\title{
PART I
}

\begin{abstract}
The next generation of nuclear reactor systems for terrestrial and space applications are designed with strong emphasis on safety, improving efficiency and economy, and reducing the security and proliferation threats. From thermal and fluid design stand points, improvements include new operational parameters as well as the use of new types of coolants and working fluids. A key element in the design of new generation of nuclear power plants is the development and use of advanced modeling tools and computer codes for faster and more accurate simulation of plant behavior under normal and abnormal operational transients. The main goal of this research is the development of a robust computational fluid dynamics and heat transfer model for engineering simulation and high resolution analysis of thermal fluid design of advanced high temperature gas cooled reactors.
\end{abstract}

At the heart of the model developed in this work is a hybrid fine and coarse mesh numerical approach to solve Navier-Stokes equations with dependent gas properties. The coarse mesh computational model is needed to simulate flow and heat transfer in the entire nuclear thermal propulsion system. The fine mesh computational fluid dynamics model is intende for use in parts of the system where high resolution and more accurate calculation of flow velocity, pressure, and temperature are needed. The computational model is intended for simulation and design analysis of very high temperature gas cooled reactors and nuclear thermal propulsion systems where properties of coolant could vary with temperature and pressure. The model uses coarse mesh points to simulate the thermal fluid behavior of the entire nuclear system. For high resolution simulation and detailed analysis of major components of the plant system, a fine mesh 
grids Computational Fluid Dynamics (CFD) model is used. The coarse mesh numerical tool is based on a simple one-dimensional integral model accounting for pressure losses and heat transfers in the reactor core and all major components. Fine computational grids are used to predict the flow map, pressure and temperature profiles in areas of the system with complex flow geometries. The fine mesh CFD model is based on two-dimensional axisymmetric Reynolds-Average Navier-Stokes solver, to account for the turbulent nature of flow in the systems. Space and time are discretized with a TVD MacCormack implicit-explicit scheme to take full advantage of the stability of the solver, even for cases where the flow properties might not be continuous. The CFD fine mesh solver offers additional flexibility with a choice of two turbulence models. First, the $\mathrm{k}-\epsilon$ model which is the most widely used model in the CFD community. It is particularly suitable for internal flows. The second choice is the Spalart-Allmaras model that has shown great performances in the scope of wall-bounded aeroacoustic flows.

The specificity of the solver comes from its ability to model the real properties of three gases of interest in gas-cooled nuclear systems, hydrogen, helium and carbon dioxide. They are modeled over a large range of temperatures and pressures. Most CFD solvers, and in particular commercial CFD codes, consider properties such as thermal conductivity, viscosity and heat capacity as constant over the domain of study. Therefore, the Navier-Stokes equations are simplified by extracting these values from the partial differential operator terms. In the case of high temperature gas cooled nuclear systems, the properties cannot be considered constant and should be modeled using temperatureand pressure-dependent correlations. Therefore, the gas properties need to be discretized in parallel with the extensive properties in the Navier-Stokes equations. The development of the real-property CFD model is motivated for the study of the low pressure thrust chamber for the Nuclear Thermal Propulsion. At low pressure and/or high temperatures hydrogen molecules start dissociating. The dissociation of hydrogen molecules greatly influences the molecular weight, the heat capacity, and viscosity. Such drastic changes in 
thermal and transport properties cannot be modeled with traditional CFD codes and are the main reason for the development of the real property CFD solver. The time-dependent Computational Fluid Dynamics solver iteratively sets the steady-state condition in the fluid systems of both the very high temperature gas-cooled reactor and the nuclear thermal rocket to validate its use for analysis of advanced nuclear power reactor systems.

The CFD solver is used to analyze portion of high temperature reactor systems cooled with helium, non-dissociated hydrogen, or with dissociated hydrogen. Results of the analysis of the helium-cooled channel for high temperature gas cooled reactor, show relatively small differences between the constant property and variable property solvers. The CFD solver is also applied to the study of the coolant channel in a nuclear thermal rocket under typical operating conditions where the pressure is not low enough to cause hydrogen dissociation. The flow, pressure, and temperature profiles obtained are quite different from the ones calculated with frozen chemistry. This validates the need for the development of CFD solver with real gas properties. Finally, the coolant channel and thrust chamber of the low pressure nuclear thermal rocket are modeled using the fine-mesh CFD solver. In both coolant channel and thrust chamber the dissociation and recombination of the hydrogen is important. Due to sharp changes in thermal properties of dissociated hydrogen the variation of the first and second gradients of these properties are not continuous. As a result, the convergence rate of the solver is very slow and quite possibly is not even achievable without further smoothing of the dissociated hydrogen property table. Therefore, cases involving significant dissociation of hydrogen did not converge. To overcome the shortcomings of using property formula, the look-up table that is implemented in the coarse-mesh solver could be used. The large number of computational mesh points and the number of steps involved in each iteration limits the use of able look-up which requires extremely high computation time and is beyond capacity of a single processor. The implementation of full hydrogen property table and the 
convergence test for the variable property CFD solver is an issue beyond the scope if the project and that needs further investigation.

Once validated on hydrogen and helium coolant channels, the real property fine-mesh solver is implemented into a coarse mesh system code. It is primarily designed for fluid simulation in nuclear thermal propulsion systems. The coupled fine and coarse-mesh solver is applied to model the entire nuclear thermal propulsion system including the reactor core where high resolution solution is obtained using the fine-mesh CFD solver. Treatment of the interfaces between the coarse-mesh and fine-mesh solvers is presented. Simulation of the system with the new coupled code shows great improvement in accuracy, speed and stability. 


\section{CHAPTER 1 \\ INTRODUCTION: ANSWERING THE NEEDS OF THE FUTURE IN THE NUCLEAR INDUSTRY AND SYSTEM MODELING}

\subsection{The Nuclear Power Scene of the 21st Century}

Research and development in the field of nuclear power production is a very dynamic field. The Generation IV International Forum was established in January 2000 to investigate innovative nuclear energy system concepts to meet future energy challenges. The members include Argentina, Brazil, Canada, Euratom, France, Japan, South Africa, South Korea, Switzerland, United Kingdom, and United States, with the OECD-Nuclear Energy Agency and the International Atomic Energy Agency as permanent observers. The forum serves to coordinate international research and development on promising new nuclear energy systems for meeting future energy challenges: The new power plants should meet the increased energy demands, while being resistant to diversion into weapons of mass destruction and safe from terrorist attacks. In 2002, the final Generation IV concepts have been selected to shape the future of nuclear energy. The international task force agreed on six nuclear technologies, which represent advances in sustainability, economics, safety, reliability and proliferation-resistance. Out of the six concepts to be developed in the scope of Generation IV nuclear reactors, two reactors are gas-cooled: the very high

temperature gas reactor (VHTR) and the gas cooled fast reactors (GFR). They are aimed at energy and hydrogen production. Both systems are helium-cooled reactors with outlet temperatures of 1,000 and 850 degrees Celsius, respectively. On June 28, 2005, the US Senate voted a wide-ranging energy policy bill which includes the authorization for reactor development work at Idaho National Lab in support of the Generation IV initiative and the nuclear hydrogen initiative. While the US Department of Energy is supporting research on several reactor concepts, priority is being given to the VHTR.

In December 2006, the National Aerospace and Space Agency (NASA) announced its will to build a base on the Moon by 2020. This implies not only manned-missions to the Moon, but also to Mars and of further planets, for which the Moon would serve 
as a launch base. The project has given a new start for research in the area of space nuclear propulsion. Started in the late 50's in the USA, the project has been progressively abandoned after the end of the cold war and the end of the space race with the Soviet Union. Nuclear space propulsion allows for faster and longer space travels. Therefore, there is a renewed and growing interest for the concept.

\subsection{Challenges in Thermal-Hydraulics}

Most of operating nuclear reactor are Light Water Reactors (LWR): As of December $200585 \%$ of worldwide nuclear plants are cooled with light water, which represents $91 \%$ of the total electrical power generated from nuclear technology [4]. Within the scope of Gen IV development, the United States is developing the VHTR. They also are leading research on Nuclear Thermal Propulsion. Both concepts use inert gases as working fluid, separating themselves from the more traditional systems operating with water. Operating characteristics of VHTR and NTP are compiled in Table 1-1 as well as the Pressure Water Reactor (PWR) and Boiling Water Reactors (BWR) for comparison. The type of coolant and operating conditions are greatly different between designs of the future (VHTR and NTP) and last generation of reactors (PWR and LWR). Besides, the difference in coolant nature (helium or hydrogen vs. water), new reactors are operated at much higher temperatures.

Over the years, the nuclear industry has largely relied on simulation tools to design systems, analyze fuel cycle, as well as studying the behavior of reactors in case of severe accidents. Besides neutronics and transport codes, thermal-hydraulics codes represent a large part of the simulation tools used by engineers and researchers. Some of the established thermal-hydraulics computer codes currently used in the nuclear industry 
are RELAP-3D ${ }^{1}, \mathrm{TRACE}^{2}$, and CATHARE ${ }^{3}$. They are power plant simulation codes, generally applied to extensive analysis of severe accidents.

Simulation of the flow behavior is obtained by solving the equations of conservation of mass, momentum and energy. The softwares use heat transfer, generic component and control systems models to describe the behavior of nuclear power plants with water (liquid and vapor) and other non compressible gases. The entire power plant is defined using volumes and junctions. Pump, turbine, pipes, pressurizers, branches and so on, are examples of volumes. Each volume is decomposed into axial nodes on which the algebraic governing equations are solved. We refer to this type of codes by the name of system codes and to the characteristics of the meshing by the appellation coarse mesh. The momentum equations solved by these codes are the Euler inviscid equations instead of the basic Navier-Stoke equations because they are much more simple. Wall friction and heat transfer are evaluated from empirical correlations and then applied to model turbulence effects. In their most updated versions, they comprise 3-D fluid dynamics modules. Due to coarse nodalization, these modules require volume-averaged coefficients for turbulent exchange of mass, energy and momentum. Such coefficients are not well known. This type of system code is not adequate in the case of complex geometries. These system codes are intended for a limited range of fluids (principally liquid water and vapor) and temperatures. They are not developed for high temperature gases.

\subsection{Issues Faced with the Current Computational Tools for Thermal-Hydraulics}

The industry needs powerful tools for the design of the future generation of nuclear reactors. Because experiments are very costly, developers of the new concepts need to rely

\footnotetext{
${ }^{1}$ LWR accident analysis code - Developed at Idaho National Laboratory

${ }^{2}$ LWR accident analysis code - Developed at Nuclear Regulatory Commission

${ }^{3}$ LWR accident analysis code - Developed at CEA-Grenoble, France
} 
mostly on accurate numerical tools. The current thermal-hydraulics codes cannot be used for the fluids and operation ranges suitable to the next generation of reactors. They need to be modified in order to rely partially on empirical correlations and models because we do not have the experimental resources to do so.

For a decade, nuclear engineers investigating original designs or particular portions of the nuclear system have been relying on Computational Fluid Dynamics (CFD). Compared to the system codes previously discussed, volumes of interest are decomposed into a fine grid in all directions to be analyzed with CFD: CFD models any flow map by solving the full set of Navier-Stokes equations on the discretized domains. We then refer to this type of solver by the name of fine mesh solver. Historically, CFD software used to be primarily developed for aerospace applications. Because it remains very versatile, applications of CFD have expanded to a large variety of engineering fields. Nuclear industry has been increasingly relying on CFD modeling since it makes possible a reduced reliance on empirical correlations and experiments.

Advances in the numerical techniques have made feasible the model of realistic reactor geometries for any type of fluid and any range of operational pressures with a few limitations still: the flow domains needs to be discretized into very small meshes. Therefore, Computational Fluid Dynamics can only model a part of the entire nuclear plant. The main reason is the extreme computational cost. It still remains a partial answer to the need in computational tools for the simulation of new generation of nuclear power generation systems involving gaseous flows at high temperature. Helium is heated and cooled over a $700 \mathrm{~K}$ range in the VHTR (cf. Table 1-1) and hydrogen undergoes a 3,000K increase in temperature in the NTP. This condition on temperature is very different from the conditions which can occur in aerospace applications: initially, the CFD techniques would consider constant properties for the gases of interest. In gaseous nuclear systems, some properties such as heat capacity for hydrogen see variations of up to $500 \%$. 
The CFD codes which are available are not built to handle such variations and can only be used with caution for the analysis of high temperature gaseous reactors.

\subsection{Objectives}

In the previous section, we highlighted the shortcomings of both thermal-hydraulics (coarse-mesh) codes and CFD (fine-mesh) codes:

- Safety codes: only allow one- and two-dimensional description of the geometry of the flow. Have limited and defined range of pressure and temperature for which they are accurate.

- CFD: computationally expensive. Have limited definition of the temperature and pressure dependence of the fluid properties.

Since system codes cannot be accurate in certain areas of the new nuclear systems and CFD codes cannot be used to study the entire system, we suggest to couple a system code with a CFD solver to combine both advantages of the two numerical tools: this technique would answer the industry needs while limiting the computational expense. On one hand, the system code and its coarse mesh would provide a fast and accurate simulation of most parts of the nuclear system. Simultaneously, Computational Fluid Dynamics numerics would be used to analyze portions of the system which exhibit particular geometries or operational conditions and for which the traditional numerical tools lack accuracy in flow prediction.

One of the objectives of the doctoral work presented here is to demonstrate that it is possible to obtain a powerful computational tool which couples fine and coarse mesh simulations for thermal-hydraulics predictions. To prove the feasibility of this project, we work on the 1-D coarse mesh code NuRok [5] which is developed at the Innovative Nuclear Space Power and Propulsion Institute at the University of Florida. This code aims at modeling the thermal-hydraulics of different types of Nuclear Thermal Propulsion systems. The entire path of the fluid flows is modeled. NuRok aims at designing NTP

systems as well as dimensioning each various component. A more detailed overview of the 
code functionality and composition is given in Chapter 7. We choose this particular code because:

- We have access to the entire source code, making it easy to add components to it.

- It is a perfect example of 1-D coarse mesh simulator which gives excellent results in certain portions of the system but lacks accuracy in some particular areas.

The second objective of this dissertation represents a more challenging task. It is to develop a CFD solver which accounts for the real properties of fluids.

\subsection{The Different Approaches}

The goal is to increase the performances of NuRok flow predictions by adding some CFD modules. Knowing that the different subroutine of the NTP simulation codes are written in FORTRAN, we needed to evaluate which technique should be adopted to couple the code to fine-mesh, CFD-type simulations.

There are several techniques to consider in order to achieve our goals. First, we could use a general purpose CFD code to model the portions of interest. The code could be FLUENT 6.2 [6] for which we already have expertise [7]. The code would be run for a various set of boundary conditions and the result of the simulation would be analyzed. We would derive correlations properly describing the behavior of the flow in the system of analysis and implement them in NuRok. This type of work has been realized by Plancher [8] in the case of the cooling channel of the SLHC core. This technique exhibits a few drawbacks: it requires a lot of preliminary work, as for running numerous simulations to cover as many operational conditions as possible. The correlations that we would develop would introduce some approximation errors. Cases where operational conditions differ from the ones investigated could arise in certain portion of the system without NuRok user being aware of it. Therefore the correlations previously derived would not be correct. This solution does not require a lot of development takedown. Indeed it proves to be a very limited answer to an increased accuracy and modularity in the nuclear system flow prediction. 
The second approach would develop the CFD models of the portion of interests. If we were to use commercial CFD codes, it would mean a lack of accuracy in the definition of the fluid properties. To have a coupling with feedback between the coarse and fine mesh simulations, the technique requires that their interfaces would be dynamically linked. However, two main problems could appear. On one hand, it seems difficult to create an interface between the two codes. On the other hand, this approach does not necessarily lead to a stable/accurate solution. Hence, we choose to develop our own CFD solver which will be particularly fitted to model gaseous nuclear systems and have the capacity to describe exactly the real properties of the gases involved.

\subsection{Overview of our Research}

This dissertation will show the differences between simulations with constant and real properties considerations. It will also show the techniques and results of coupling efforts between one-dimensional fine-mesh system code and fine-mesh computational fluid dynamics solver. 
Table 1-1: Characteristics and operating conditions of different nuclear systems [1]

\begin{tabular}{|c|c|c|c|c|c|c|c|}
\hline $\begin{array}{l}\text { type of } \\
\text { reactor }\end{array}$ & coolant & phase & $\begin{array}{l}\text { working } \\
\text { fluid }\end{array}$ & $\begin{array}{l}\text { inlet } \\
\text { temp }(K)\end{array}$ & $\begin{array}{l}\text { exit } \\
\text { temp }(K)\end{array}$ & $\begin{array}{c}\text { pressure } \\
(\mathrm{MPa})\end{array}$ & $\begin{array}{l}\dot{m} \\
(\mathrm{Mkg} / \mathrm{h})\end{array}$ \\
\hline pressurized & & & & & & & \\
\hline $\begin{array}{l}\text { water reactor } \\
\text { boiling }\end{array}$ & water & $\begin{array}{l}\text { liquid } \\
\text { liquid }\end{array}$ & water & 300 & 603 & 15.3 & 65 \\
\hline $\begin{array}{l}\text { water reactor } \\
\text { gas-cooled }\end{array}$ & water & vapor & water & 269 & 559 & 7 & 47 \\
\hline reactor & helium & gas & helium & 337 & 828 & 5 & 5 \\
\hline NTP & hydrogen & SGL & hydrogen & 22 & 3200 & $0.01-5$ & $5.10^{-4}$ \\
\hline
\end{tabular}




\section{CHAPTER 2 \\ APPLICATION FUNDAMENTALS}

To better understand the challenges of the doctoral work, the chapter gives a short description of the domains of application of interests as well as a quick presentation of the major tool used through the dissertation: computational fluid dynamics. Applications of the research work include nuclear space propulsion and more particularly Nuclear Thermal Propulsion. Basic principles of a low-pressure thrust chamber for combination to space propulsion are also explained.

\subsection{Space Propulsion and Nuclear Thermal Propulsion}

Historically, space rockets have been powered by chemical propulsion. Specific impulse (Isp), also called specific thrust, is a measure of the rocket performance. It is defined as the level of thrust divided by the propellant mass flow rate (Equation 2-1).

$$
I s p=\frac{\text { thrust }}{\text { propellant mass flow rate }} \simeq \frac{v_{\text {exhaust }}}{g}
$$

where $v_{\text {exhaust }}$ is the velocity at the exit of the thrust chamber and $g$ is the gravitational constant.

Chemical propulsion is the only technology used to launch rockets as of today. Its performances are limited compared to other systems under investigations such as space nuclear propulsion: chemical propulsion Isps are no greater than $475 \mathrm{~s}^{-1}$. In contrast to traditional chemical propulsion, nuclear propulsion could provide Isps at least twice as big. First estimations evaluated that nuclear propulsion would reduce a trip to Mars from 600 days to about 200 days. Space nuclear propulsion is hoped to create a breakthrough in space exploration, allowing manned missions to Mars or rocket exploration of outer planets.

One of the current approaches to space nuclear propulsion is the nuclear thermal propulsion (NTP), which stands as the main focus of this study. In the NTP design, cold hydrogen is heated by a compact nuclear core. Temperature of the fluid at the exit of 
the reactor is about $3,000 \mathrm{~K}$. Hydrogen then enters the thrust chamber where it expands. Finally, it accelerates to velocities as large as 10,000 m/s.

The investigation of the performances and design of the NTP started in 1955 with a joint effort between NASA and the Atomic Energy Commission [9]. It was terminated in 1973 due to the change in national priorities. Over the twenty years of active work on the project, nineteen cores were investigated combining various types of solid fuel and core design.

The general layout of a NTP system is shown Figure 2-1. Liquid hydrogen is stored in a tank at a temperature of 20-22 K. Over the years, it as been established by the nuclear engineering community that redundancy of the turbo-machineries is the first step for plant safety. In cases when one of the turbo-pump system would fail, the redundant systems would help safe operation of the system. The same principle is applied to NTP and the main hydrogen flow line is separated into at least two parallel and identical systems. First, hydrogen flows through a pump which extracts the fluid from the tank. Since the fluid is heated, compressed and is in a single gaseous phase at the exit of the pump. Hydrogen cools down the walls of the nozzle and of the core reflector. Then, it enters turbines which are coupled to the first pumps. The different flow lines are recombined before they enter the core of the propulsion. At the exit of the nuclear core, temperature of the fluid reaches 3,000 K. The very hot hydrogen then enters the thrust chamber, a diverging-converging nozzle. The gas expands and accelerates, thrusting the rocket.

The Innovative Nuclear Space Power and Propulsion Institute at the University of Florida developed a system code to model the flow of the gas in NTP systems [10]. The 1-D code aims at sizing the different parts of the system for given operational thermal power and core exit pressure. It has been developed such that a large variety of NTP designs can be studied, as well as a panel of system organizations. This coarse mesh simulation tools will be referred in this dissertation as NuRok (Nuclear Rocket). The skeleton of the code relies on the resolution of the momentum equation. However, it 
required experiment-based relations for characteristics such as friction factor or Nusselt number. NuRok users can monitor pressure and temperature at any point of the NTP system of consideration.

This code is very competitive to get a good qualitative idea of the performances of a given system within a set of operating conditions. Since it is a one-dimensional representation of the NTP, it gives results on the Isp, mass flow rate and other characteristics within seconds. Several factors limit the accuracy of the results obtained. First, the one-dimensional representation of highly non-symmetric geometries biases the simulation. Besides, relations on friction factor or Nusselt number for example would require experimental data on hydrogen in temperature and pressure conditions comparable to the ones of NTP systems. However, experiments with hydrogen-gas for temperatures as high as 3,000 $\mathrm{K}$ is costly and hazardous: high temperature hydrogen is extremely flammable and corrosive. Hence, very few information on the behavior of hydrogen in conditions comparable to the ones of NTP is available. It is then difficult to estimate if the relations available in the literature for different gases or operating conditions apply to our system.

\subsection{The Low-Pressure Thrust Chamber}

The previous section highlighted the main interest of space nuclear propulsion. We believe that using nuclear propulsion will increase space rockets Isps. Typically, the nuclear core reactor exit temperature and pressure are equal to 3,000 $\mathrm{K}$ and 1850 psi (about $12 \mathrm{MPa}$ ), respectively [8]. In the case of a lower pressure with constant temperature, the molecules of hydrogen $\left(\mathrm{H}_{2}\right)$ start dissociating. As they recombine, there is extra energy which is liberated in the gas. The dissociation energy of a molecule of hydrogen $\mathrm{H}_{2}$ is equal to $4.52 \mathrm{eV}$ [11]. This can be observed when considering the heat capacity $c_{P}$ which is discontinuous at the pressure/temperature of dissociation [12]. The properties of hydrogen and dissociated hydrogen will be described more in detail in Chapter 4 . However, the behavior of the hydrogen molecule as it dissciates/recombines 
leads to the following statement: We believe the dissociation/recombination effects of the hydrogen molecule at high temperature and low pressure is transmitted to the gas under kinetic energy. Hence, if we operate a Nuclear Thermal Propulsion system at low pressure, hydrogen molecules will dissociate in the core. As the flow passes through the thrust chamber, hydrogen temperature decreases, molecules recombine, transmitting kinetic energy to the flow. Hence, If we operate the core at low pressure, we think that the exhaust velocity of the hydrogen flow through the system will be greatly increased, leading to high values of the Isp, and thus increase the performances of the system.

\subsection{Computational Fluid Dynamics: An Overview}

Fluid (gas and liquid) flows are governed by partial differential equations (PDEs) which represent conservation laws for the mass, momentum, and energy. The system of equations is referred to as the Navier-Stokes equations. It can only be solved numerically. To get the flow map, the solution also requires an equation of state, for example ideal-gas law if it applies. Computational Fluid Dynamics (CFD) is the technique of replacing such PDE systems by a set of algebraic equations which can be solved using computers. A CFD solution is obtained after performing the following steps:

- Discretization of the domain

- Discretization in time and space of the partial differential equations

- Iteration to obtain a converged numerical solution

- Post-processing

Once a discrete solution in space and time is obtained a flow map is provided: it consists of the values of density, velocity components, energy and pressure or temperature at every mesh point. In most flow patterns, the geometry is complex and parts of the Navier-Stokes equation components require modeling (e.g. turbulence effects). The accuracy of the numerical solution depends on the quality of the discretization technique and relevance in the model of choice. Several numerical methods and algorithms have been proposed and developed over the last three decades. Despite several decades of 
investigation no single method or approach is robust enough for simulation of any type of flow. A compromise between simplicity, ease of implementation, accuracy and computational efficiency ought to be made. Appropriate approximations and models are developed to reach the highest quality possible of the information generated by CFD.

We now detail the differences between CFD simulation and experiment. Traditionally, CFD gives an insight into flow patterns that are difficult or expensive to study using traditional experimental techniques. Experiments give a quantitative description of the flow for one quantity at a time (e.g. temperature, pressure or, velocity), at a given number of points, for a laboratory-scale model and for a limited range of problems and operating conditions. Errors can arise from measurement errors or flow disturbances by the probes. Similarly, numerical simulations result in a quantitative prescription of the flow for all desired quantities with a high resolution in space and time, for the actual flow domain and virtually any realistic problem and set of operating conditions. However, CFD does not replace the measurements completely since numerical techniques require benchmarking in order to assess their accuracy.

For the type of systems we want to analyze here, simulation is a much more adapted method. CFD is much faster, broader and cheaper technique than experimentation. 


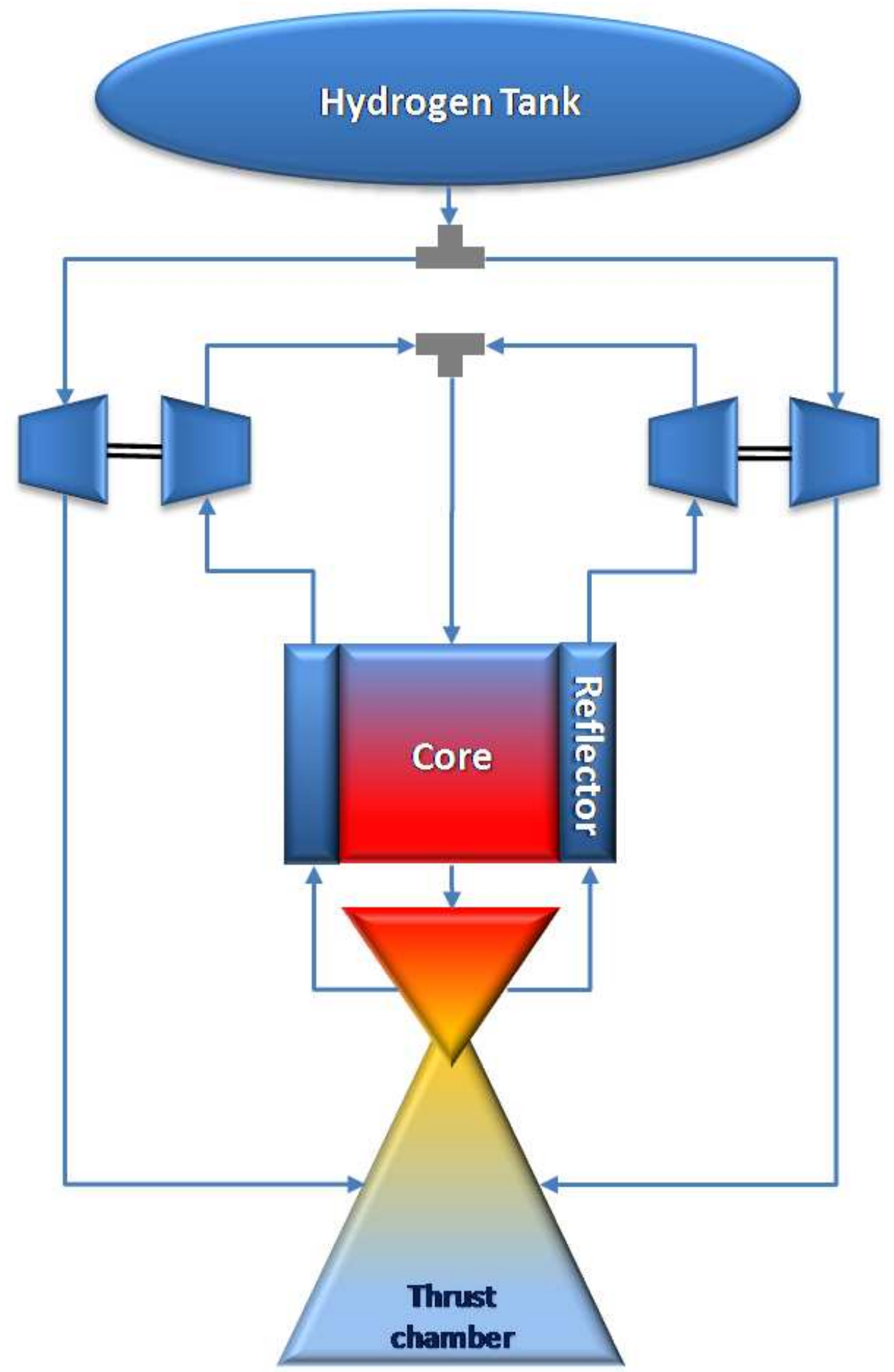

Figure 2-1: The serial cooling system (NERVA type) 
CHAPTER 3

\section{A SURVEY ON STATE-OF-THE-ART COMPUTATIONAL FLUID DYNAMICS DEVELOPMENTS AND APPLICATIONS}

Since the early 1970s, commercial software packages have become available, making CFD an important component of engineering design and analysis [13]. Ten years later, the use of commercial CFD software started to become accepted by major companies around the world rather than their continuing to develop in-house CFD codes. Historically, the nuclear industry has relied on CFD capabilities in its first stages of development: CFD was applied to safety analysis and core reload design of nuclear power plants. Over the last twenty years dramatic improvements in both numerical techniques and computing power have offered the possibility of performing large-scale, high-fidelity core thermal-hydraulics analysis.

The most commonly used commercial CFD codes are the finite volume solvers FLUENT [6], CFX [14] and Star-CD [15]. These codes offer a large variety of solvers to discretize and solve the Navier-Stokes partial differential equations which describe most flow behaviors. They also offer a large variety of modeling capabilities, including but not restricted to turbulence, radiative heat transfer and two-phase modeling. To a large degree, these codes offer very similar performances and modeling options.

Freitas [16] has compiled a comprehensive benchmarking of the different commercial CFD codes. These benchmark problems included: the backward-facing step problem, the uniform flow past a circular cylinder, the three-dimensional shear-driven cavity flow (all three laminar flows), the turbulent flow around a square cross-section tube and the developing turbulent flow in a 180 degree elbow. The main drawbacks of the Freitas [16] study were the lack of independence and completeness. The study was performed by CFD code vendors and to a large extent did not include turbulence modeling. The $\mathrm{k}-\epsilon$ model [17] is the most commonly used turbulence model in practical industrial applications. A detailed formulation of $\mathrm{k}-\epsilon$ model is described in Chapter 5. It is this particular model which accounted for turbulence modeling in the benchmark study of 
Freitas. The benchmark test cases in Freitas study are extreme in terms of application of this particular model. The study concluded that in general, the different commercial CFD codes produce similar results. The Freitas study, at the time, recommended an increase in the spacial discretization order and a wider variety of turbulent models to choose from. These features have been added to most commercial CFD codes over the last decade, but due to highly competitive nature of the business, CFD vendors have not agreed to participate in another benchmark exercise.

Many of the nuclear reactor research programs around the world are focused on the development of numerical tools for safety analysis of Light Water Reactors (LWR) with emphasis on increasing the simulation accuracy. In 2003, Yadigaroglu [18] presented the trends in LWR safety analysis. The article underlined the shortcomings of the traditional system codes and proposed to move toward multi-dimensional analysis by taking advantage of single and two-phase detailed CFD capabilities. The main issue addressed by the paper was the limitation in computing power and skills for use of CFD in practical problems. The main areas of interest for use of CFD capabilities are the primary loop and containment of nuclear power plants. To date, CFD analyses performed in nuclear power plant systems included boron dilution in the primary loop of Pressurize Water Reactors (PWR), mixing of various gases, stratification of containment pools, etc.

Boyd [19], [20] analyzed a 1/7th and full-scale steam generator inlet plenum mixing during a PWR severe accident. One-sixth of the inlet plenum was modeled with FLUENT 6.0 using the Reynolds Stress Model (RSM) associated with non-equilibrium wall function. Experiments on the $1 / 7$ th scale model validated the accuracy of the turbulence model for the inlet plenum geometry and flow. However, numerical results of the study exhibited an non-physical oscillatory behavior of the plume for the geometry associated with a symmetry plane. This problem had already been identified with the use of FLUENT's on several test cases. The non-physical behavior can be attributed to the shortcomings of 
the earlier versions of FLUENT. Besides a few discrepancies in the area of the symmetry plane, computed results were in good agreement with experimental data.

Keheley [21] modeled a boron dilution transient during the shut down phase of a PWR plant. The analysis was based on STAR-CD's Algebraic Multigrid Solver with the Re-Normalization Group (RNG) k- $\epsilon$ turbulence model. Simulation results were compared to test data and it was shown that temperature dependent properties and buoyancy effects modeling could increase the accuracy of the simulation. The Keheley's work demonstrated that STAR-CD, could adequately model a typical boron dilution transient in PWR cores.

Tinoco [22] showed the feasibility of analysis of the steam line break transient in Boiling Water Reactors (BWR) using STAR-CD. Since turbulences do not have time to develop in such fast transients, the laminar solver was considered. Such a transient actually occurred in Unit 1 and 2 at the Forsmark nuclear power plant. The incident was caused by defective welding and led to the replacement of the core grids. Tinoco's CFD simulation helped to develop a better understanding of incidents which could occur while the plant is in operation.

Behavior of the water flow and heat transfer around rod bundles in LWR cores is also a major subject of interest in reactor safety study. The rod bundle is a standard geometry in LWRs because of the fuel pin arrangement. Modeling of rod bundles in LWR cores is a great test case for assessment of CFD codes. References [23] and [24] present a wide variety of experimental results involving flow and heat transfer in LWR fuel bundles. Using the rod bundle test data, Tzanos [25] demonstrated the shortcomings of the STAR-CD and it's associated linear k- $\epsilon$ models for the simulation of such problems in PWR cores. Tzanos simulation results showed a significant discrepancy with experimental data. This was particularly true in areas of flow deflection such as spacer grids. The results highlighted the need for other RANS turbulence models or LES.

In et al. [26] led a more detailed analysis of the same problem by investigating various RANS turbulence models with the CFX code. These turbulence models included 
the standard k- $\epsilon$ model, the non-linear quadratic Speziale k- $\epsilon$ model, the SSG (Speziale, Sarkar, and Gatski) model and the RSM- $\omega$ model. It is important to note that not all of these models are available in the original source code, and the solver had to be modified to allow for incorporation of such models. The RSM model showed best agreement with the test results by accurately accounting for the anisotropy of turbulence and by predicting, with great accuracy, the turbulence-driven secondary flows. The RSM model also enabled computation of Reynolds stresses, wall shear stresses and wall temperature values very close to experimental results. However, the RSM model lacked accuracy in prediction of flow and heat transfer in tight lattice rod bundles. Other turbulence models had to be investigated for cases where there is a large difference in geometrical scales. In's simulation results for flow around a bundle with split-vane spacer [27] showed that the standard and Speziale k- $\epsilon$ models gave the most accurate predictions. Results of In et. al. studies [26]-[27] proved that the choice of turbulence model must be carefully considered depending on the flow situation.

The k- $\epsilon$ model usually remains the safest choice in turbulence modeling. Hence, in 2001, a consortium of twelve European partners started the project [28] under sponsoring of the European Commission. ECORA stands for Evaluation of Computational Fluid Dynamics Methods for Reactor Safety Analysis. The project aimed at assessing the performances of numerous CFD codes (CFX, FLUENT, Saturne, STAR-CD and TRIO-U) for simulating flows in the primary system and containment of nuclear reactors. The project also highlighted and defined CFD code improvements that were necessary for nuclear engineering applications. The interest in the three-dimensional flow effects in some reactor components arose from the inability of one-dimensional system codes to predict accurately flow behavior in specific areas of the reactor systems. Examples of systems under investigation were:

- fluid-fluid mixing in the cold leg and downcomer taking place during a small break LOCA, 
- coolant mixing in the rod-to-rod and rod-to-wall gaps,

- effects of spacers on coolant mixing,

- mixing of both forced and buoyant jets/plumes, jet impingement on curved surfaces, plunging jets, and interaction of parallel jets/plume,

- boron-dilution events,

- contact condensation in the two-phase stratified steam-water flow.

All the work we just cited testifies of the dynamic activity surrounding CFD modeling in the area of LWR safety analysis. It is of great importance since we saw that $85 \%$ of the nuclear power plants worldwide are Light Water Reactors. However, the system of the future which is the most investigated today in the world and in the United States in particular is the VHTR. In 2004, Schultz [29] simulated the mixing of Helium in the lower plenum of a prismatic Gas-Cooled Reactor. The standard k- $\epsilon$ model of FLUENT 6.1 was considered along with buoyancy modeling. The predicted hot and cold temperatures were within 1.5 to $2 \mathrm{~K}$ of the measured values. This result shows the good accuracy of the simulation. Comparison was made possible by McEligot report [30] which compiled a few experiments that have been developed at INL to assess the performances of the CFD codes in the case of the VHTR. More recently, Ahmad et al. [31] studied the 3-D distribution and pressure drop at the inlet plenum and rising channel of a pebble-bed modular high temperature reactor with CFX-5.7.1. The fluid was modeled by an incompressible and isothermal flow and once again the $\mathrm{k}-\epsilon$ model accounted for turbulent effects. The model was not benchmarked but Ahmad formulated recommendations for similar CFD studies. However, considering an incompressible flow in the VHTR seems like a very limiting assumption.

CFD studies in the nuclear industry are not limited to applications to the primary loop of power plants. CFD is of great help for other fields of study related to nuclear applications. For example, Lee [32] used CFX capabilities to model the heat generated by radioactive decay of dry spent fuel in a canister. The analysis required to account 
for the buoyancy-induced natural convection and for the laminar flow of both Helium and Nitrogen. The boundary condition of consideration at the wall was a thermal flux derived from the power density generated by radioactivity. The computed results were consistent with natural convection phenomena found in literature. Great resolution of the computational grid close to the walls helped improve accuracy of the solver.

Therefore, CFD is widely used in the nuclear industry for simulations of very detailed portions of nuclear systems. The review shows that CFD codes can be used to model flow conditions of almost any nuclear system designs from traditional PWRs to designs of the near future such as VHTR. CFD simulations are limited by the size of the domain of consideration: because a power plant geometry is composed of so many different length scales, a potential meshing of the entire system would require several million of computational cells as we will see in the next section. Also, the review highlights the shortcomings of some of the classical turbulence models available in commercial CFD codes. When leading our own CFD analysis, much attention will be given to the turbulent effects and their modeling in the systems we consider.

\subsection{System Coupling}

For all the previous applications we reviewed, only targeted portions of the systems for analysis with CFD codes. On the opposite, Weber et al. [33] developed a high fidelity representation of a current generation PWR with STAR-CD. The study modeled the entire core for which the grid was composed of 240 million CFD computational zones. Calculations required 50 hours of computation on 200 IBM processors (in 2000) to get the steady-state solution. The analysis proved that CFD can be used to assess fluid-dynamics and thermo-mechanical issues that were not possible with more simplified approaches. Even if such an extensive study is possible, it does not remain realistic as we want to study transients of the core. This demonstrates again that CFD analysis in nuclear reactor systems should be restricted to small, targeted portions. In order for us to simulate the 
entire Nuclear Thermal Propulsion system on a thermal-hydraulics point of view, we will have to consider coupling codes.

Lots of recent international efforts have been focused toward the development of coupled simulation codes which combine advantages of different computational softwares: rapidity of 1-D system codes and accuracy of CFD codes. Research on coupling between system-codes and CFD started in the late 1990's with the publication of Yoon and Suh's work [34]. It consisted of an indirect coupling applied to the Korean next generation reactor. A CFX simulation analyzed in detail the flow for the direct vessel injection, while the bulk of the system was simulated with the thermal-hydraulics code TRAC. The system code returned a boundary condition which became the inlet boundary for the CFD simulation. This coupling technique is limited to steady-state cases.

Accident analyzes generally require full-coupling, capable of handling transients. With that in mind, the Idaho National Laboratory (INL) started a co-operative project: couple FLUENT with RELAP5-3D [35]. The major part of the nuclear system is simulated with RELAP. One or more particular portion of the system is modeled with FLUENT. In the particular example of Weaver the system is composed of a simple linear association of volumes with a by-pass. The by-pass is composed of a heated channel modeled with CFD and a simple pipe modeled with RELAP. RELAP starts calculating the map flow in the first cells until it reaches the by-pass. The properties computed by RELAP at the inlet of the by-pass are input as the inlet boundary condition for the FLUENT model. FLUENT is run and returns a set of properties at the exit of the pipe which are implemented back into RELAP as inlet conditions for the rest of the system. This type of coupling is referred to as dynamic coupling. It is the optimum coupling for obtaining accurate solutions: this coupling combines the advantages of the two types of codes.

The nuclear industry also needs similar coupling between CFD and neutronics codes. This type of work is currently under investigation. The NURESIM project [36] is a very promising collaboration which aims at combining a pin-based Monte-Carlo 
or deterministic transport calculation with a full CFD - Thermal-hydraulics core-wide sub-channel simulation code. This work should be noted however not relevant to this research.

Most of the coupling difficulties when using commercial CFD codes arise from the data interpretation, from the module interface. Such coupling should be made easier by a standardization of the commercial CFD code architecture and module interfaces [37].

The first part of the review shows that commercial CFD codes when used properly can give satisfying results to compute portions of flows in nuclear systems. However, Weber's study [33] showed that it is not realistically possible to simulate the transient of an entire system because of the computational cost. Several attempts are made at coupling system codes with commercial CFD codes but the module interface makes the task really difficult. Also, the commercial codes are not built to consider large variations of properties with temperatures and pressure. For all the previous reasons, we decided to develop out own solvers as we explained in the introduction. Our solver should be applicable to compressible high Reynolds number flows over all ranges of Mach numbers (subsonic, transonic and supersonic flows) as well as turbulent flows. We will now review the numerical methods developed which apply to the type of flow we want to model.

\subsection{Numerical Techniques for CFD Solvers}

\subsubsection{Considerations on Compressible Flows}

At high Reynolds number, the convective effects are predominant compared to diffusive effects. Therefore, the fluid behaves like an inviscid flow [38]. The governing equations for a viscous fluid are the system of Navier-Stokes equations: they reduce to the Euler equations in the case of inviscid flow. The numerical techniques for the two types of equations are very similar except for the discretization of the shear stress which arise in viscous flows. For compressible and supersonic flows, there is formation of shocks, expansion fans and contact discontinuities. Specific numerical schemes should be considered to capture properly pressure and properties discontinuities. 
Numerical techniques developments on Euler equations started in the early 1950s with the work of Courant et al. [39], Lax and Friedrichs [40]. Lax-Friedrichs methods are easy to evaluate and smooth, however they are excessively dissipative [41]. The milestone for the modern development is to be found in the work of Lax and Wendroff [42] which resulted in the type of solver named after them. Their main characteristic is to be second-order accurate, space-centered and explicit in time.

A variant of this method, expanded to the system of Navier-Stokes equations, was developed later: it is the widely-used explicit MacCormack scheme [43]. Most of the work in the early 1960s happened at the Courant Institute on hyperbolic systems. The numerical schemes tended to exhibit non-physical oscillations and it was difficult to obtain converged steady-state solutions. At the same time, in Russia, Godunov introduced the concept of representing the flow as piecewise constant in each computational cells and solving a Riemann solver at each interface [44]. The scheme was first order accurate and avoided non-physical features such as expansion shocks: expansion shocks cannot happen because they violate the Third Law of Thermodynamics, meaningly entropy can only increase $(\Delta S \geq 0)$. Because Godunov's pioneering work was not translated as it was published, it took time for the West to acknowledge the numerical technique [45]. However very accurate, the scheme was very costly in terms of calculations of the complex fluxes at the interfaces.

In 1981, Roe [46] developed a technique which enabled a practical and efficient implementation of Godunov-type schemes: he introduced the concept of locally linearizing the governing equations through a mean value Jacobian: his work still has a major impact on modern computational techniques. Roe's technique belongs to the Approximate Riemann Solvers class, along with Osher's method [47]. Roe's concept is based on a flux-difference splitting scheme and yields a single-point numerical shock structure for stationary normal shocks. 
ENO (Essentially Non-Oscillatory) scheme of Harten et al. [48] is another example of widely used flux-splitting solvers. Godunov's work also recognized that numerical schemes might benefit from distinguishing various wave speeds leading to characteristic-based schemes. Early high-order characteristic-based solvers used flux vector-splitting, the most famous being the scheme of Steger and Warming [49]. It still led to oscillations near discontinuities.

The Monotone Upstream-centered Schemes for Conservation Laws (MUSCL) of Van Leer [50] extended Godunov's scheme to higher orders: the scheme was second order accurate in space thanks to the introduction of flux-limiters. Limiters are functions which smoothes the numerical solutions in areas where high order scheme would normally create oscillations. Their first use dates back to 1973 with the work of Boris and Book [51] who demonstrated perfect propagation of discontinuities. Harten [52] demonstrated that Total Variation Diminishing (TVD) schemes (see Section 5.1.10) are monotonicity-preserving and enable oscillation control in the solution of non-linear problems. An other very significant work is the Runge-Kutta algorithm by Jameson [53] which is based on an alternative-direction implicit approximate factorization.

During the eighties, development of compressible Euler solvers was an essential step toward simulation of the more complex flows.It was made possible thanks to techniques such as combination of upwinding, artificial methods, finite element or finite volume approximations and flux limiting concepts, for example. We now present the MacCormack methods which are of great importance for our research. It belongs to the family of block methods which consist in solving all the equations simultaneously. It usually requires more computations at each grid point but improves dramatically the convergence rate [54].

\subsubsection{Discussion on the MacCormack Methods}

In 1969, MacCormack published pioneering work for solving the system of Navier-Stokes equations in compressible, high Reynolds number flows [43]. The solver was explicit, second-order accurate and used the predictor-corrector method. Its performances were 
well-proven. However, explicit schemes are convergent and stable as long as the time step is small enough to satisfy the stability condition. In 1975, MacCormack published an improved version of his own technique [55].

Continuity of his work led to a combined implicit-explicit MacCormack scheme in 1981 [56]. Explicit schemes are always numerically stable and convergent but usually more numerically intensive than the explicit method. The explicit predictor-corrector method which is faster is used over most of computational domain. However, it is not adequate for points in the flow at which the local CFL number exceeds the stability limit [57]: the method adds an implicit procedure to the predictor-corrector sequence. The method is implicit in nature and thus allows a much larger time marching step size.

The method posses three advantages over fully-implicit methods. First, the method uses two-point, one-sided differences which lead to block bidiagonal systems, easier to invert that block tridiagonal systems found in traditional methods. Secondly, the method uses inviscid jacobians and corrects them using representative viscous terms added to the eigenvalues. This maintains stability while avoiding the expensive calculation of the viscous jacobian. Finally, the algorithm allows the implicit step to be skipped in regions where the stability restriction condition is satisfied. The expressions given for the predictor and corrector steps are adequate for applications with the finite volume method.

Snyder [58] compared the original 2-2 MacCormack scheme from 1969 [43] to a 2-4 modified version of the method and other similar explicit finite difference methods. The methods where benchmarked against a Gaussian pulse diffusion and the shock tube: the two test cases study non-linear wave propagations of an inviscid flow, i.e. they require to solve for the system of Euler equations only. Both experiments are well documented and allow detailed validation of numerical techniques. The methods included artificial damping or filtering models to reduce spurious oscillations. The 2-2 MacCormack scheme is second order accurate in space and time. The 2-4 scheme is fourth order accurate in space: the spacial accuracy is improved by increasing the stencil size from three to five. 
A MacCormack-Baldwin [59] damping function was added to both numerical methods to suppress spurious pulses. The study shows that the 2-2 MacCormack is the fastest method of all investigated. However, it gives spurious oscillations and is highly diffusive. The 2-4 method captures better the sharpness of the shock but largely overshoots the value of the discontinuity. The analysis concludes that each method has its own unique feature and that the order of accuracy of a method does not necessarily is a good indicator of the method performances: To reduce oscillations in areas of discontinuities one might prefer a 2-2 MacCormack over a 2-4 MacCormack even if the first is more dissipative.

After the release of MacCormack implicit-explicit predictor-corrector scheme [56], some analyzes showed that the implicit MacCormack method is unreliable such that the steady-state solutions depend on time increments' [60]. The severity of such dependence was assessed by Ong [61] in a comprehensive study in 1987. The scheme was also compared to the fully-implicit scheme of Beam and Warming [62]. The study was validated over a supersonic turbulent flow past a two-dimensional compression corner. Turbulence was simulated by the algebraic turbulent eddy-viscosity of Baldwin and Lomax [63]. The study concluded that the steady-state solution of the explicit-implicit method is relatively insensitive to the Courant number, therefore the steady-state appeared to be independent of the size of the time step. Moreover, the accuracy of the steady-state solution using MacCormacks hybrid algorithm was comparable to that of the Beam-Warming method for all cases, and was observed to reduce the computing time by a factor of up to three. Lawrence [57] also investigated the performances of the implicit-explicit form of MacCormack technique: results of the simulation of a laminar flat-plate boundary-layer and hypersonic laminar flow over a 15-degree compression corner were compared to experimental results and the Beam-Warming scheme [62]. The results compared pretty well with published results and experimental data. However, the authors identified a source of error in the boundary treatment, particularly visible in the flat-plate simulation: the reflective condition at the boundary led to instabilities at the beginning of 
the iteration. Therefore, the authors recommended the use of explicit boundary condition treatment in the case of the MacCormack simulation.

Although not very sophisticated, MacCormack techniques are perfectly satisfactory for many fluid flow applications especially in the case of 2D flows [64]. The MacCormack schemes are particularly adapted for the applications of interest in this dissertation: they are fast and easy to implement, and the accuracy is satisfactory for the type of flow we investigate.

\subsubsection{Considerations on Turbulence}

All of the techniques we just described can be applied to the system of Navier-Stokes equations. However the Navier-Stokes equations are only valid for laminar flows. Most practical engineering flows are turbulent. In turbulent flows, large and small scales of continuous energy spectrum proportional to the size of the eddy motions are mixed. Eddies are overlapping in space, larger ones carrying smaller ones. In this process, turbulent kinetic energy transfers from larger to smaller eddies and the smallest eddies eventually dissipate into heat through molecular viscosity. A typical 0.1 by $0.1 \mathrm{~m}$ flow domain with relatively high Reynolds number might contain eddies down to 10-100 $\mu \mathrm{m}$. To compute all length scale a grid of $10^{9}$ up to $10^{12}$ mesh points would be required to describe all scale lengths [65]. Engineers need access to viable tools capable of representing the effects of turbulence. Here are the three methods which are commonly used:

- Direct Numerical Simulation (DNS)

- Reynolds Averaged Navier-Stokes (RANS)

- Large Eddy Simulations (LES)

In Direct Numerical Simulation (DNS), resolution of the grid is chosen such that all turbulent scales, large and small, are resolved. This is a deterministic method, which requires prohibitive computer code and cannot undertake large-scale industrial problems. Since turbulence is characterized by random fluctuations, statistical methods are being extensively studied. In the context of the Reynolds-Averaged Navier-Stokes 
(RANS) method, the system of conservation equations is time-averaged. The various flow properties are decomposed into mean and fluctuating components. It results in new variables appearing in the governing equations: the average value of the product of velocity components fluctuations. These terms are models. It corresponds to modeling of all turbulence scales. Therefore, RANS method does not require as much mesh refinement as for DNS. The Large Eddy Simulation (LES) technique is a compromise between the DNS and the RANS method. For this technique, large eddies are computed and small eddies are modeled. Small scale eddies are associated with the dissipation range of turbulence and are simpler to model than in RANS. This method requires more refinement than RANS since the large eddies are computed but not as much as the DNS because the small-scale turbulence is modeled. In DNS, the Kolmogorov microscale [66] defines the size of the meshes and in 3-D the number of grid points ends up being equal to $N=R e^{9 / 4}$. Therefore, DNS is not adapted to the type of solvers we aim at building. The development of the LES methods remains particularly marginal because the computation of the large scale eddies requires an amount of computational resources which were not available until a few years ago. Moreover the nuclear industry is just beginning to introduce this technique for analysis of turbulent flows.

Most of the time, engineers are interested in information about the time-averaged properties of the flow. This is particularly true for the studies presented in this dissertation. Hence, the RANS technique is often privileged and historically, has been the most developed technique, thus is very well documented. The majority of the CFD simulations for nuclear reactor applications we previously quoted rely on the $\mathrm{k}-\epsilon$ model for turbulence considerations. Even if some research work show the limitations of this model in certain cases [25], it is not clear if this model is suitable for all researches. Besides, the previous review showed the importance of accounting for the real properties of the fluids [29]. However, the commercial CFD codes which are traditionally used have limited capabilities in real properties modeling. Moreover Direct Numerical Methods, Large Eddy simulations 
and other advanced CFD methods have not yet found many applications in nuclear engineering. However, they will become more and more use as precise two-phase simulation with interface tracking is one of the main research focus. Also since all the flows in nuclear engineering applications are internal, modeling of the fluid close to the walls is of great importance. The industry would gain from the use of a combination of advantages of both RANS and LES turbulence modeling in high Reynolds number flows. This technique known as hybrid RANS-LES solves for different scales of turbulence while being still computationally affordable. In this method unsteady RANS is used near walls and away from walls LES is used. The matching between RANS and LES takes place in the inner log-region ${ }^{1}$. Unfortunately, this model is still in its development phase and not available in commercial CFD codes: there are still some issues on the area of transition between RANS and LES treatment of the turbulent viscosity [67]. Georgiadis [68] solved this problem by using a RANS and a LES mixing-length model. However, when applied to compressible turbulent mixing layers it only enables predictions of the main characteristics of the boundary layers and the authors themselves concluded that there is large room for calculations improvement.

Therefore, the hybrid RANS-LES methods for turbulence modeling are in a too early stage for us to consider in our solvers. Hence, we will build our CFD solvers using the RANS method that is widely documented and primarily used for flow analyzes related to the nuclear engineering field. In the review of CFD analysis in nuclear systems, the models of predilection are primarily the $\mathrm{k}-\epsilon$ and RSM models. The $\mathrm{k}-\epsilon$ seems very well-adapted to the VHTR coolant channel study. It is a two-equation model and therefore is one of the RANS models which requires relatively low computational capabilities. It has shown good performances in the study of subsonic pipe flows, which is our interest for the coolant

\footnotetext{
${ }^{1}$ Stefan Wallin: Turbulence Modeling and Flow Control - Seminar - University of Florida - April, 262007
} 
channel. In the case of the NTP thrust chamber, we study a transonic flow which is wall bounded. As we detail in the next section, the Spalart-Allmaras model [69] seems to be more adapted in the particular case of the thrust chamber.

\subsubsection{Discussion on the Spalart-Allmaras Model}

In 1994, Spalart and Allmaras developed a turbulence model for applications to wall-bounded transonic flows: the model was based primarily on empiricism and on dimensional analysis arguments [70]. It is a very recent model, but we review the work which has been done on the validity of the model to determine if it is applicable to our particular case of thrust chamber.

Paciorri [71] investigated the validity of the Spalart-Allmaras turbulence model for hypersonic flows. The model was validated on a variety of test cases: flat plate turbulent boundary layer flow, hypersonic wind-tunnel flow, a Mach number equals 5 flow over a hollow cylinder and a Mach number equals 6.8 flow over a hyperbolic flare. The study concluded that the model, implemented in a finite volume solver with a finite difference technique, overall gave good predictions of the flow behavior. Exception was made in cases which involved turbulent separation The author recommended the use of the model for attached flows, which is the case of the thrust chamber flow we investigate. Fink and Pirzadeh [72] also investigated the validity of their solver on a flat-plate boundary-layer, using the Spalart-Allmaras technique to model turbulence. The Euler equations were discretized using Roe's scheme and flux limiters Superbee or Minmod. Bother Euler equation and the Spalart-Allmaras transport equations were discretized using an implicit backward Euler time-stepping method. Therefore, it allowed a loose coupling between the different equations. The Spalart-Allmaras model was coupled with a wall function formulation to eliminate the need for resolving the flow in the sublayer portion of a turbulent boundary layer. The paper showed that this technique gave good results both in the flow field and the boundary layer, and had the advantage of reducing the computing memory requirement and improving overall convergence. Bonfiglioli et al. [73] studied 
the two-dimensional steady transonic turbo-machinery flow, where the eddy viscosity was modeled with the Spalart-Allmaras model. The analysis proved the good behavior of the turbulent model to predict the transonic flow. Finally, Cavicchi [74] led an extensive study of nine turbulence models applied to the secondary flow in an accelerating $90^{\circ}$ elbow and the performances of each models were assessed with the exit passage vortex. The Spalart-Allmaras model gave the best predictions along with the Baldwin-Lomax and the Shear Stress Transport models.

In conclusion, we observe that CFD has become one of the preferred simulation tools in thermal-hydraulics studies for portions of nuclear systems. The nuclear industry relies mostly on commercial CFD-codes, but these codes exhibit a few drawbacks: They were not aimed at modeling large pressure and temperature variations which lead inevitably to major property variations. Also, a very critical issue in CFD simulations is turbulence modeling: the turbulence models often offered in commercial codes were developed for aeroacoustic flows. Some studies ([16], [26], [27]...) aimed at evaluating the validity of such models for nuclear applications. It resulted that, besides its relative simplicity, the $\mathrm{k}-\epsilon$ turbulence model showed good performances. We then will be able to use this model for most of our simulations. However, in the case of the NTP thrust chamber, our review shows that a simpler model, the Spalart-Allmaras model, would give better results while being computationally less expensive. Also, we reviewed the main numerical techniques for compressible flows which are competitive in shocks and discontinuities capture. Usually, commercial codes only allows one type of solver for this type of flow: for example, FLUENT 6.1 is limited to a third order MUSCL scheme. Although very accurate in most flow types, the user might want more flexibility. In our case, we want to get results as accurate as possible but with an emphasis on calculation rapidity. In the previous review, we showed that the MacCormack methods might be the one most relevant for our study. In the scope of the development of CFD solvers for VHTR and NTP study, this review helps us make informed choices of each of the different techniques. 


\section{CHAPTER 4 \\ MATERIAL PROPERTIES}

The present analyses focus on the hydrogen flow in NTP, helium and carbon dioxide flow in gas-cooled reactors. The fluids are gases in the operating conditions we consider. They undergo a large range of pressure and temperature. To get accurate modeling of the flow the CFD solvers need to consider a variety of gases over a large range of operating conditions. Therefore, meticulous modeling of the properties behavior with temperature and pressure is critical. We model the density, heat capacity, viscosity, thermal conductivity and molecular weight. All the gas data which are available give discrete values for various sets of operating conditions. We would like to have a continuous description of all properties to avoid computationally expensive table reading. Besides, continuous definition better describes the property gradients involved in the governing equations. This section aims at describing the properties models as polynomial functions of $\mathrm{T}$ and $\mathrm{P}$. The models should match the experimental data within $5 \%$ for our simulations to give a realistic model of the actual fluid flows. Models for hydrogen, helium and carbon dioxide properties are given. The properties of considerations are:

- Density

- Heat Capacity

- Viscosity

- and Thermal Conductivity

\subsection{Hydrogen}

Hydrogen is considered for applications in the space nuclear propulsion only. For example, for the square lattice honeycomb design of the NTP, when the core thermal power is equal to $600 \mathrm{MW}$ hydrogen operating conditions in the system range from $22 \mathrm{~K}$ and 2 bars in the storage tank to $3,000 \mathrm{~K}$ and 110 bars [8]. This is the starting point for the range of operating conditions. We saw in section 2.2 that we expect great performances of the rocket thrust chamber if the system is operated at low pressure. 
Therefore, we want to derive all hydrogen property correlations over the range:

$$
\left\{\begin{array}{c}
22<T<3,500 \mathrm{~K} \\
0.1<p<150 \text { bars }
\end{array}\right.
$$

This range covers all the operating conditions for the possible NTP designs, plus a safety margin.

\subsubsection{Note on Dissociation}

When the core is operated at low pressure, there is the possibility that the molecules of hydrogen start dissociating as the fluid temperature reaches a threshold in temperature [75]. The dissociation follows the scheme:

$$
H_{2} \rightarrow H+H
$$

The level of dissociation is plotted Figure 4-1 as a function of temperature for different pressures. Dissociation can only occur at temperatures greater than 1,500 K. At a given temperature, the lower the gas pressure the more molecules dissociate. Dissociation primarily impacts the molecular weight of the gas and its heat capacity. The molecular weight of hydrogen at room temperature and atmospheric pressure is $M=2.016 \frac{\mathrm{g}}{\mathrm{mol}}$ As the gas start dissociating the statistical average of the gas molecular weight drops. The values of the molecular weight is obtained from Vergaftig [12]. Figure4-2 shows the evolution of the heat capacity with temperature when there is a high level of dissociation ( $\mathrm{p}=0.1$ bar) and when the impact of dissociation is negligible ( $\mathrm{p}=150 \mathrm{bars}$ ). At the lowest pressure the heat capacity of the dissociated gas abruptly increases whereas at 150 bars, the heat capacity remains roughly constant. At 0.1 bars, the heat capacity reaches a maximum at $T=3,000 \mathrm{~K}$. 


\subsubsection{Thermo-Physical Properties}

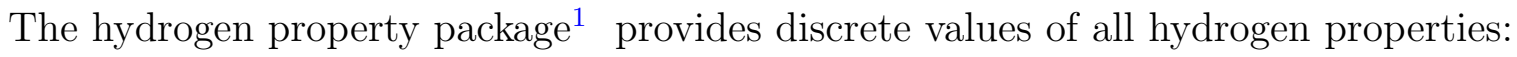
enthalpy, entropy, speed of sound, density, thermal conductivity, viscosity, specific heat and specific heat ratio. For temperatures below 3,000 K, data are based on the National Bureau of Standards. Above 3,000 K and up to 10,000 K data are obtained from NASA computer programs for para- and dissociated hydrogen [76] and the National Institute of Standard and Technology. The property data provided by the hydrogen package are the best available as of today. A computer routine developed by Chen [77] conveniently interpolates thermodynamic and transport properties of hydrogen within the temperature range above. Different techniques are used to develop the hydrogen properties package: Linear interpolation (LI), natural cubic spline interpolation (NCSI), and multiple polynomial curve-fitting with polynomial bridging between the curve fits. The package allows the user to get hydrogen properties for a discrete set of temperature and pressure. For use in the CFD solver, we would like to have a continuous description of the different properties as a function of temperature and pressure to avoid cpu-expensive table reading and be able to describe as well as possible property gradients which appear in the fluid governing equations. Each set of properties is now curve-fitted to obtain a correlation in good agreement with experimental data (about 5\%).

- Density and molecular weight:

By definition, an ideal gas is a hypothetical gas with molecules of negligible size that exert no intermolecular forces. A molecule of hydrogen, composed of only two atoms, and moreover the two lightest ones, should be very close to an ideal gas. For an ideal gas, the density is defined by

$$
\rho=\frac{p}{R T}
$$

where $p$ is the pressure, $R$ the perfect gas constant and $T$ the temperature. A comparison between real thermo-physical data and ideal gas approximation is presented Tables 4-1 to 4-5. The level of dissociation of the hydrogen molecules is

\footnotetext{
${ }^{1}$ http://www.inspi.ufl.edu/data/h_prop_package.html
} 
taken from Vargaftik [12] and shown Figure 4-1. We compare density values obtained from Given [78] to the theoretical density calculated with the ideal gas law corrected for molecular weight variations due to the dissociations of hydrogen molecules. The relative errors are compiled in the last column of the tables. Over most of the temperature and pressure range the ideal gas law applies well since the relative errors remain smaller than $3 \%$. At the extreme range of pressure, when the pressure is small $(P=0.1$ bars and $T>3,000 \mathrm{~K})$ the relative errors increases up to $30 \%$. The error is too large to consider the ideal gas law is accurate under these conditions. Figure 4-3 shows the difference between the data from Given [78], the data from Vargaftik [12] and those computed from the ideal gas law: density is plotted as of function of the inverse of temperature. It seems that the values from Given at high temperature are not accurate: the error has been identified as coming from a digit inversion when the density values were input in the code. The graph shows the good agreement between the ideal-gas law model and the density values from Vergaftik. Therefore, the ideal-gas law correctly describes the hydrogen density behavior for all ranges of pressure and temperature.

All hydrogen thermo-physical properties (besides density that we just discussed) are implemented in the solver through correlations which are a function of both pressure and temperature. Those correlations are polynomial functions of the two extensive properties.

\section{- Heat Capacity:}

The heat capacity is very sensitive to both pressure and temperature and is defined as follow:

$$
c_{P}(\mathrm{~kJ} / \mathrm{kg} . \mathrm{K})=a_{1}(p) T^{4}+a_{2}(p) T^{3}+a_{3}(p) T^{2}+a_{4}(p) T+a_{5}(p)
$$

where $T$ is in Kelvins, $p$ in bars and

$$
\left\{\begin{array}{l}
a_{1}(p)=0.560 p^{-0.4} \\
a_{2}(p)=-2.630 p^{-0.35} \\
a_{3}(p)=4.349 p^{-0.30} \\
a_{4}(p)=-2.730 p^{-0.25} \\
a_{5}(p)=20.57 p^{-0.05}
\end{array}\right.
$$

This model introduces errors of up to $30 \%$ for areas of great dissociation as seen Figure 4-4. It is too large compared to our goal of 5\%. Also, the model lacks accounting for the decrease in heat capacity at $p=0.1$ bars when the temperature is greater than $3,000 \mathrm{~K}$. However, it gives a good trend of the property behavior, in particular when dissociation occurs. It is not a perfect model, but it has the great advantages of being continuous, which is very important for the development of the CFD solver. Note, the formulation above is for heat capacity expressed in $\mathrm{kJ} / \mathrm{kg} . \mathrm{K}$. The SI units are J/kg.K.

- Viscosity: 
For pressures greater or equal to 1 bar, viscosity of hydrogen is pressure-independent and can be modeled by:

$$
\mu(\mathrm{kg} / \mathrm{m} . \mathrm{s})=-1.44 * 10^{-12} T^{2}+1.69 * 10^{-8} T+4.64 * 10^{-6}
$$

where $T$ is the temperature expressed in Kelvins. We can use this approximation in all ranges of pressure and temperature: viscosity calculated with Equation 4-3 remains within $5 \%$ of the measured viscosity. Figure 4-5 shows the good agreement between the model and the experimental data.

- Thermal Conductivity:

For temperatures smaller than $2,000 \mathrm{~K}$ thermal conductivity of hydrogen is pressure independent and linear with temperature. The following linear approximation

$$
k(\mathrm{~W} / \mathrm{m} . \mathrm{K})=3.54 * 10^{-4} \mathrm{~T}+9.13 * 10^{-2} \quad T<2,000 \mathrm{~K}
$$

leads to an error on the calculated values no greater than $2 \%$.

When temperature is greater than $2,000 \mathrm{~K}$ thermal conductivity is pressure-dependent. An interpolation is given below, where the pressure is expressed in bars. This formulation gives an error no greater than $6 \%$ compared to the measured values

$$
\begin{gathered}
k(\mathrm{~W} / \mathrm{m} . \mathrm{K})=a_{1}(p) * T^{2}+a_{2}(p) * T+a_{3}(p) T \geq 2,000 \mathrm{~K} \\
\left\{\begin{array}{l}
a_{1}(p)=2.43 * 10^{-11} * p^{2}-9.28 * 10^{-9} * p+1.46 * 10^{-6} \\
a_{2}(p)=-1.04 * 10^{-7} * p^{2}+3.98 * 10^{-5} * p-5.87 * 10^{-3} \\
a_{3}(p)=1.11 * 10^{-4} * p^{2}-4.24 * 10^{-2} * p+6.72
\end{array}\right.
\end{gathered}
$$

In Equations 4-4 and 4-5 temperature $T$ is expressed in Kelvin and the pressure $p$ in bars.

- Implementation of the dissociation effects - the case of hydrogen molecular weight: Since the ideal gas law is an accurate model for hydrogen density modeling, we use

$$
\rho=\frac{p}{\frac{\Re}{M(p, T)} T}
$$

to derive the density at any point of the domain. The universal gas constant $(\Re)$ is equal to $8314.3 \mathrm{~J} / \mathrm{kg}$.mole. $\mathrm{K} . M(p, T)$ is the molecular weight of hydrogen which varies with temperature and pressure.

No simple correlation was found which could describe accurately the variation of molecular weight with variable operational conditions (Figure 4-1).

Therefore, we develop a linear extrapolation between each discrete point. If we look for the molecular weight at pressure $p$ and temperature $T$ such that

$$
\left\{\begin{array}{l}
p 1 \leq p<p 2 \\
T 1 \leq T<T 2
\end{array}\right.
$$


we compile the molecular weight at $p$ and $T$ such that:

$$
\left\{\begin{array}{l}
\omega_{t}=\frac{T-T 1}{T 2-T 1} \\
M(T, p 1)=\omega_{t} * M(T 2, p 1)+\left(1-\omega_{t}\right) * M(T 1, p 1) \\
M(T, p 2)=\omega_{t} * M(T 2, p 2)+\left(1-\omega_{t}\right) * M(T 1, p 2) \\
\omega_{p}=\frac{p-p 1}{p 2-p 1} \\
M(T, p)=\omega_{p} * M(T, p 2)+\left(1-\omega_{p}\right) * M(T, p 1)
\end{array}\right.
$$

With the correlations above on temperature and pressure dependance of hydrogen properties, we are able to implement real gas properties in the CFD solver with an accuracy within $5 \%$ of the literature data.

\subsection{Helium}

Helium gas is the coolant of predilection for the VHTR. Table 4-6 compiles VHTR operating conditions and features. Helium circulates in a closed loop. Typically, the coldest point of the loop, at the inlet of the core, is at a temperature of $490{ }^{\circ} \mathrm{C}$. In order to get the best efficiency out of the system, helium is heated up to $950-1,000^{\circ} \mathrm{C}$. The usual operating pressure is estimated at $7.12 \mathrm{MPa}$. The range of operating conditions over which we derive the helium properties correlations is therefore:

$$
\left\{\begin{array}{l}
1<p(\text { bars })<140 \\
200<T\left({ }^{\circ} \mathrm{C}\right)<1,500
\end{array}\right.
$$

Helium atomic weight is constant through the range of pressure and temperature we

consider and equal to $M=4.003 \mathrm{~g} / \mathrm{mol}$. The specific heat ratio is then $R=\frac{\Re}{M}=2077$. We refer to Vargaftig [12] for helium thermal-properties.

- Density:

Since helium is a mono-atomic gas, it should behave closely to an ideal gas. We compare the experimental data to the ideal-gas law within range 4-8. The results are presented in Table 4-7 and show that the ideal-gas law properly describes the behavior of helium density: the theoretical data fall within $4 \%$ of the real values.

- Heat capacity:

Vergaftig [12] only lists heat capacity of helium at low temperature. We refer to Lemmon (et. al.) [79] for temperatures ranging from 200 to 1,500 K and pressures 
between 1 and 100 bars. The value of helium heat capacity can be modeled by a constant, independent of temperature, with great accuracy (within $0.2 \%$ of the experimental data). Helium heat capacity in not dependent on $T$ and $p$ because helium is a mono-atomic gas. Compared to hydrogen there is no dissociation occuring, which has been identified as the source of heat capacity variations. We model helium heat capacity by:

$$
c_{P}(\mathrm{~J} / \mathrm{g} . \mathrm{K})=5.191
$$

- Viscosity:

The correlation to model viscosity as a function of pressure and temperature is

$$
\mu(\mu \text { Pa.s })=a_{1}(T) * p+a_{2}(T)
$$

where $T$ is expressed in Kelvins and $p$ in bars and

$$
\left\{\begin{array}{l}
a_{1}(T)=2.25 * 10^{-2} T^{-1.12} \\
a_{2}(T)=3.69 * 10^{-4} *+9.04 * 10^{-2}
\end{array}\right.
$$

The values obtained with Equation 4-10 fall within $0.4 \%$ of the experimental data for temperatures within range 4-8. The good agreement between the model and the data can be seen Figure 4-6.

- Thermal Conductivity:

Values of helium thermal conductivity are pressure and temperature dependent. We define:

$$
k\left(\mathrm{~W} /(\mathrm{m} . \mathrm{K})=a_{1}(T) * p+a_{2}(T)\right.
$$

where,

$$
\left\{\begin{array}{l}
a_{1}(T)=4.92 * 10^{-12} T^{2}-1.20 * 10^{-7} T+8.41 * 10^{-5} \\
a_{2}(T)=2.75 * 10^{-4} T+7.43 * 10^{-2}
\end{array}\right.
$$

where $T$ is expressed in Kelvins an $p$ in bars. Through the range of interest for the VHTR the calculated values are within $5 \%$ of the experimental data and the comparison between the two can be seen Figure 4-7.

\subsection{Carbon Dioxide}

A few VHTR designs in the early stage of development consider using carbon dioxide $\left(\mathrm{CO}_{2}\right)$ as a coolant and working fluid. For example, carbon dioxide is investigated in some pebble-bed reactors configurations. One of the criteria of the code development is its modularity. Therefore, it is important for our solvers to model carbon dioxide and thus get an estimation of its performances as a coolant compared to helium. We derive the 
behavior of the gas for the same range of pressure and temperature as helium (range 4-8).

We use the data provided by Lemmon (et.al.) [79].

- Density:

The ideal gas law gives good results to model carbon dioxide density. Isothermal experimental and computed densities are plotted as a function of pressure (Figure 4-8). The model does not give good results for temperatures of $400 \mathrm{~K}$. For temperatures equal or greater than $800 \mathrm{~K}$ the computed results fall within $2 \%$ of the experimental data. To derive the ideal gas law model, we use $M=44.011 \mathrm{~g} / \mathrm{mol}$ and $R=\frac{\Re}{M}=$ 188.9 .

- Heat Capacity:

Between 400 and 1,100 K the heat capacity increases with temperature at lower pressure. For pressures greater than 30 bars, heat capacity first decreases as temperature increases and then increases. To account for this phenomenon we use this higher order modeling:

$$
c_{P}(\mathrm{~J} / \mathrm{g} . \mathrm{K})=a_{1}(p) T^{4}+a_{2}(p) T^{3}+a_{3}(p) T^{2}+a_{4}(p) T+a_{5}(p)
$$

where $T$ is expressed in Kelvin $p$ in bars and

$$
\left\{\begin{array}{l}
a_{1}(p)=10^{-12}\left[6.61 * 10^{-4} p^{2}+3.07 * 10^{-2} p-0.179\right] \\
a_{2}(p)=10^{-8}\left[-2.18 * 10^{-4} p^{2}-1.07 * 10^{-2} p+8.06 * 10^{-2}\right] \\
a_{3}(p)=10^{-5}\left[2.62 * 10^{-4} p^{2}+1.41 * 10^{-2} p-0.159\right] \\
a_{4}(p)=10^{-2}\left[-1.38 * 10^{-4} p^{2}-8.35 * 10^{-3} p+0.175\right] \\
a_{5}(p)=2.66 * 10^{-4} p^{2}+1.94 * 10^{-2} p+0.447
\end{array}\right.
$$

The model leads to calculated values of the heat capacity within $5 \%$ of the NIST data. Both calculated and experimental data are plotted Figure 4-9

- Viscosity:

Over the range of operating conditions $4-8, \mathrm{CO}_{2}$ viscosity is pressure independent and varies linearly with temperature as seen Figure 4-10. We model carbon dioxide viscosity by:

$$
\mu(\text { Pa.s })=3.43 * 10^{-8} T+7.01 * 10^{-6}
$$

where $T$ is expressed in Kelvin. The modeled values fall within $3 \%$ of the NIST data. They are also computed on Figure 4-10.

- Thermal Conductivity:

Carbon dioxide thermal conductivity can be modeled by:

$$
k\left(\mathrm{~W} /(\mathrm{m} . \mathrm{K})=a_{1}(p) * T+a_{2}(p)\right.
$$


where, $T$ is in Kelvins ad $p$ in bars.

$$
\left\{\begin{array}{l}
a_{1}(p)=-6.10 * 10^{-8} p+7.48 * 10^{-5} \\
a_{2}(p)=7.64 * 10^{-5} p-4.51 * 10^{-3}
\end{array}\right.
$$

The model gives results within $3 \%$ of experimental data. Plot of modeled and experimental data are given Figure 4-11

Development of real property CFD solver requires continuous descriptions of all thermal-physical properties of the gas of considerations. Temperature- and pressure-dependent correlations are derived for hydrogen, helium and carbon dioxide for density, heat capacity, viscosity and thermal conductivity. All the modeled values fall within $5 \%$ of the data from our literature sources. The major remark after this study is that all three gases behave like ideal gases. 


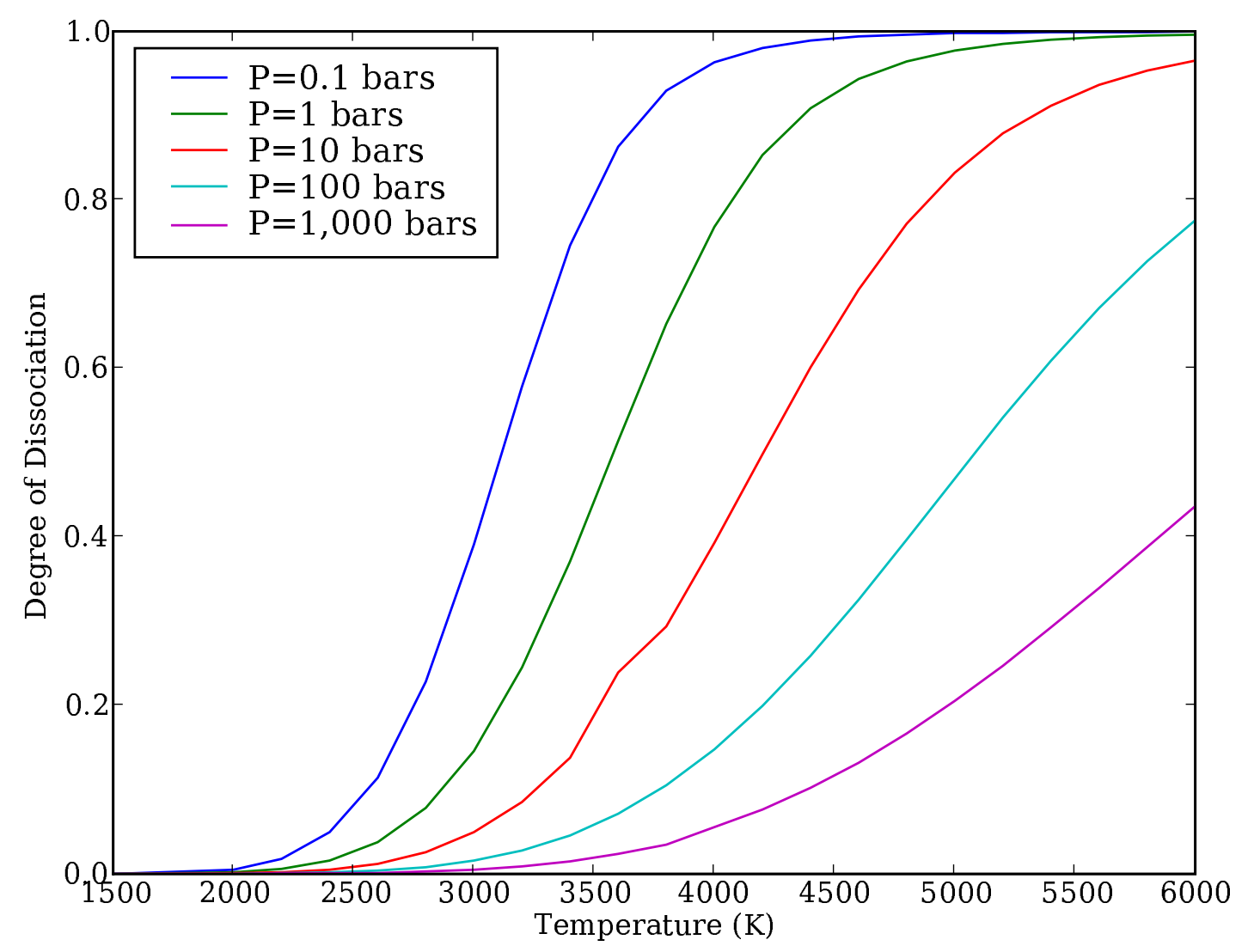

Figure 4-1: Level of hydrogen molecule dissociation as a function of temperature 


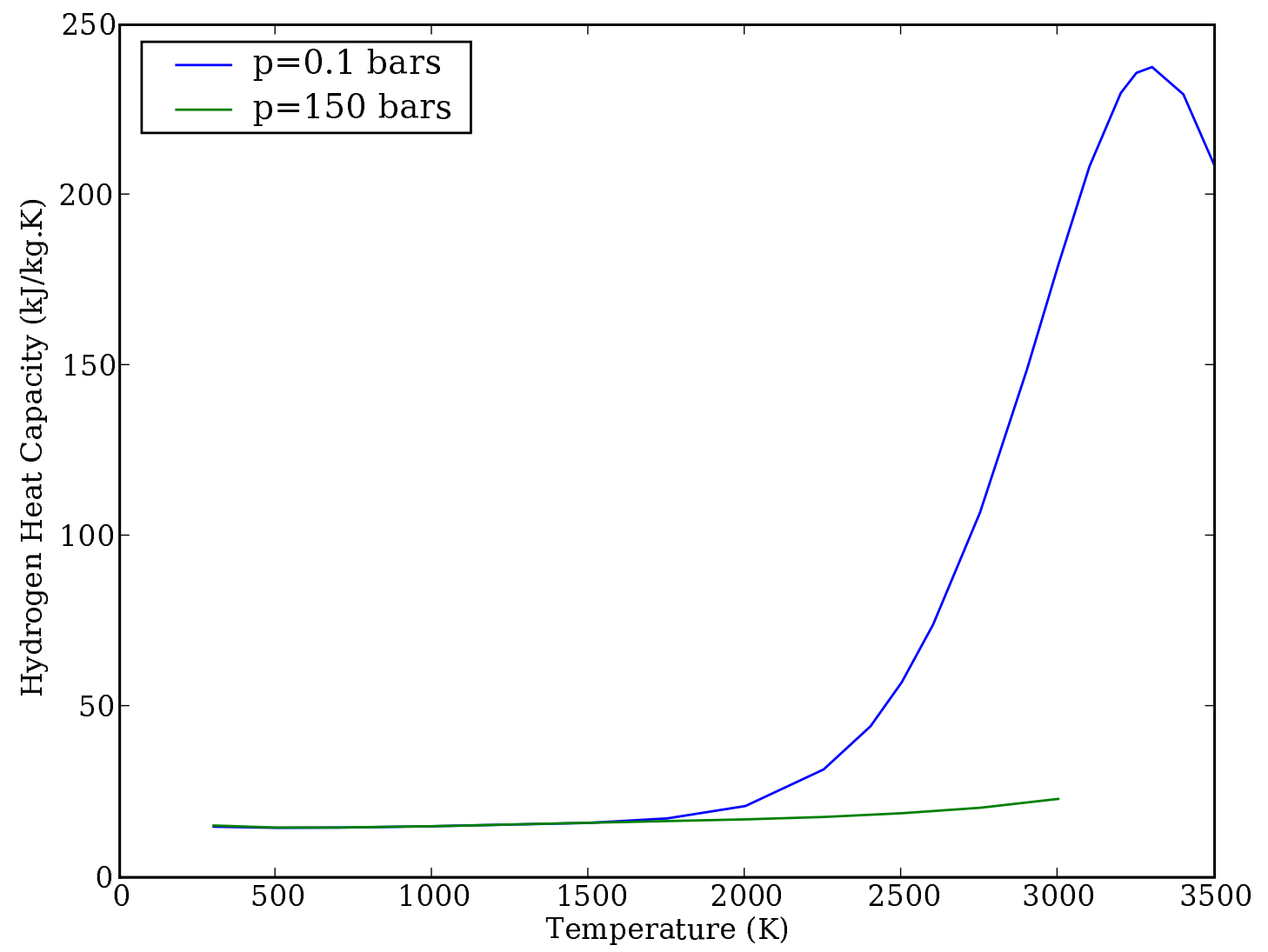

Figure 4-2: Hydrogen heat capacity vs. temperature at low and high pressure (0.1 and 150 bars)

Table 4-1: Error on the density formulation for $\mathrm{P}=0.1 \mathrm{bar}$

\begin{tabular}{llllll}
\hline $\mathrm{P}(\mathrm{bar})$ & $\mathrm{T}(\mathrm{K})$ & $\mathrm{M}(\mathrm{g} / \mathrm{mol})$ & $\rho$ - IGL & $\rho$ - compiled & error $(\%)$ \\
\hline 0.1 & 1500 & 2.016 & $1.62 * 10^{-3}$ & $1.60 * 10^{-3}$ & 1.3 \\
0.1 & 1700 & 2.016 & $1.43 * 10^{-3}$ & $1.39 * 10^{-3}$ & 2.3 \\
0.1 & 1900 & 2.013 & $1.27 * 10^{-3}$ & $1.25 * 10^{-3}$ & 2.2 \\
0.1 & 2000 & 2.011 & $1.21 * 10^{-3}$ & $1.20 * 10^{-3}$ & 0.8 \\
0.1 & 2200 & 1.998 & $1.092 * 10^{-3}$ & $1.10 * 10^{-3}$ & 0.7 \\
0.1 & 2400 & 1.966 & $9.85 * 10^{-4}$ & $1.00 * 10^{-3}$ & 1.5 \\
0.1 & 2800 & 1.786 & $7.67 * 10^{-4}$ & $8.00 * 10^{-4}$ & 4.3 \\
0.1 & 3000 & 1.622 & $6.50 * 10^{-4}$ & $7.00 * 10^{-4}$ & 7.6 \\
0.1 & 3200 & 1.433 & $5.39 * 10^{-4}$ & $3.91 * 10^{-4}$ & 27.4 \\
0.1 & 3600 & 1.146 & $3.83 * 10^{-4}$ & $3.30 * 10^{-4}$ & 13.7 \\
\hline
\end{tabular}




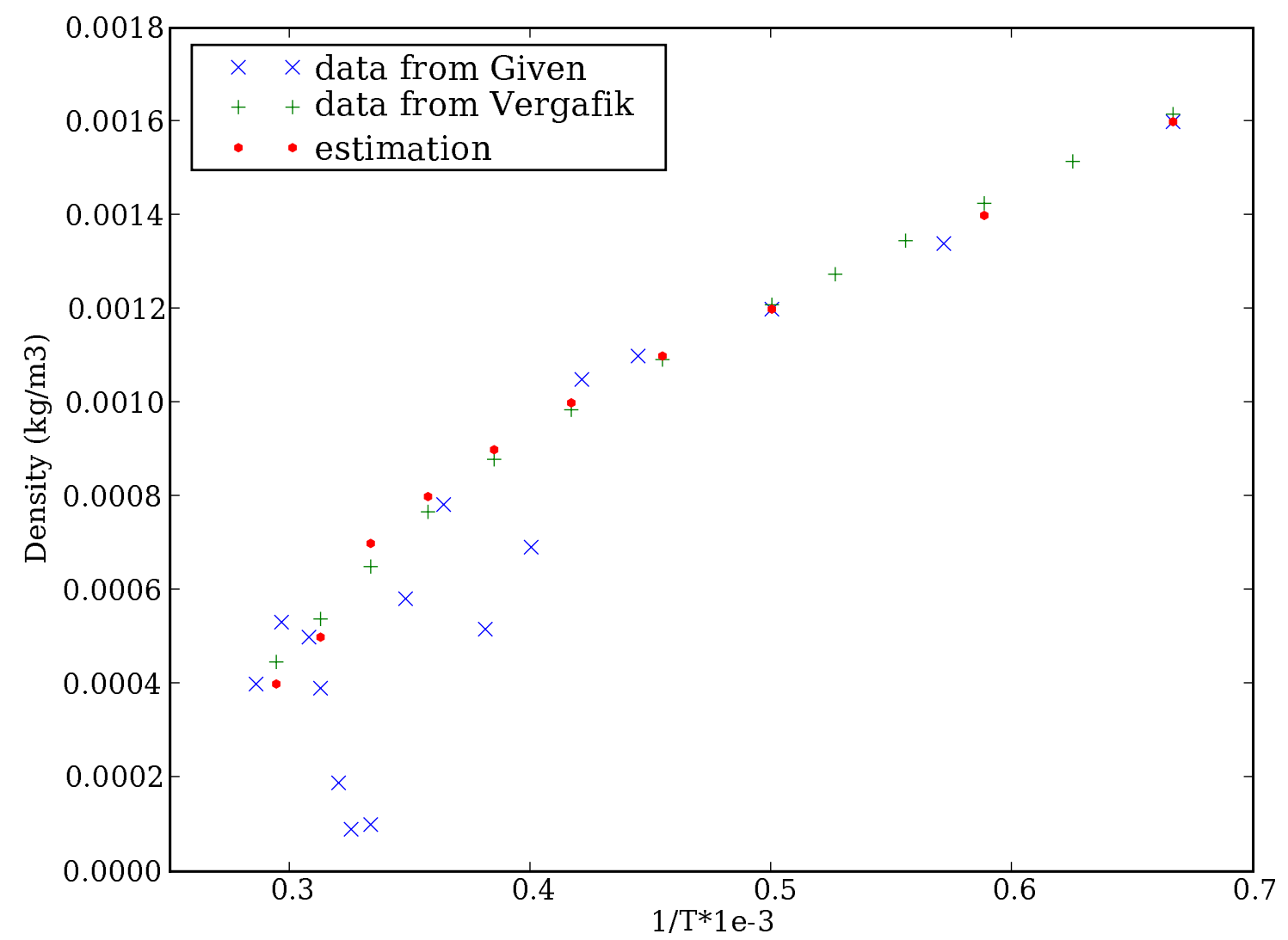

Figure 4-3: Hydrogen density at 0.1 bar and high temperature. Differences between the literature data, Given [78] and Vergaftig [12] and the data computed with the ideal gas-law

Table 4-2: Error on the density formulation for $\mathrm{P}=1$ bar

\begin{tabular}{llllll}
\hline $\mathrm{P}(\mathrm{bar})$ & $\mathrm{T}(\mathrm{K})$ & $\mathrm{M}(\mathrm{g} / \mathrm{mol})$ & $\rho$ - IGL & $\rho$ - compiled & error $(\%)$ \\
\hline 1 & 1700 & 2.016 & $1.43 * 10^{-2}$ & $1.43 * 10^{-2}$ & 0.4 \\
1 & 1900 & 2.015 & $1.28 * 10^{-2}$ & $1.28 * 10^{-2}$ & 0.0 \\
1 & 2000 & 2.014 & $1.21 * 10^{-2}$ & $1.21 * 10^{-2}$ & 0.1 \\
1 & 2200 & 2.010 & $1.10 * 10^{-2}$ & $1.10 * 10^{-2}$ & 0.1 \\
1 & 2400 & 2.000 & $1.00 * 10^{-2}$ & $1.00 * 10^{-2}$ & 0.2 \\
1 & 3000 & 1.869 & $7.49 * 10^{-3}$ & $7.50 * 10^{-3}$ & 0.1 \\
1 & 3200 & 1.769 & $6.65 * 10^{-3}$ & $6.84 * 10^{-3}$ & 2.9 \\
1 & 3600 & 1.498 & $5.00 * 10^{-3}$ & $5.07 * 10^{-3}$ & 1.4 \\
\hline
\end{tabular}




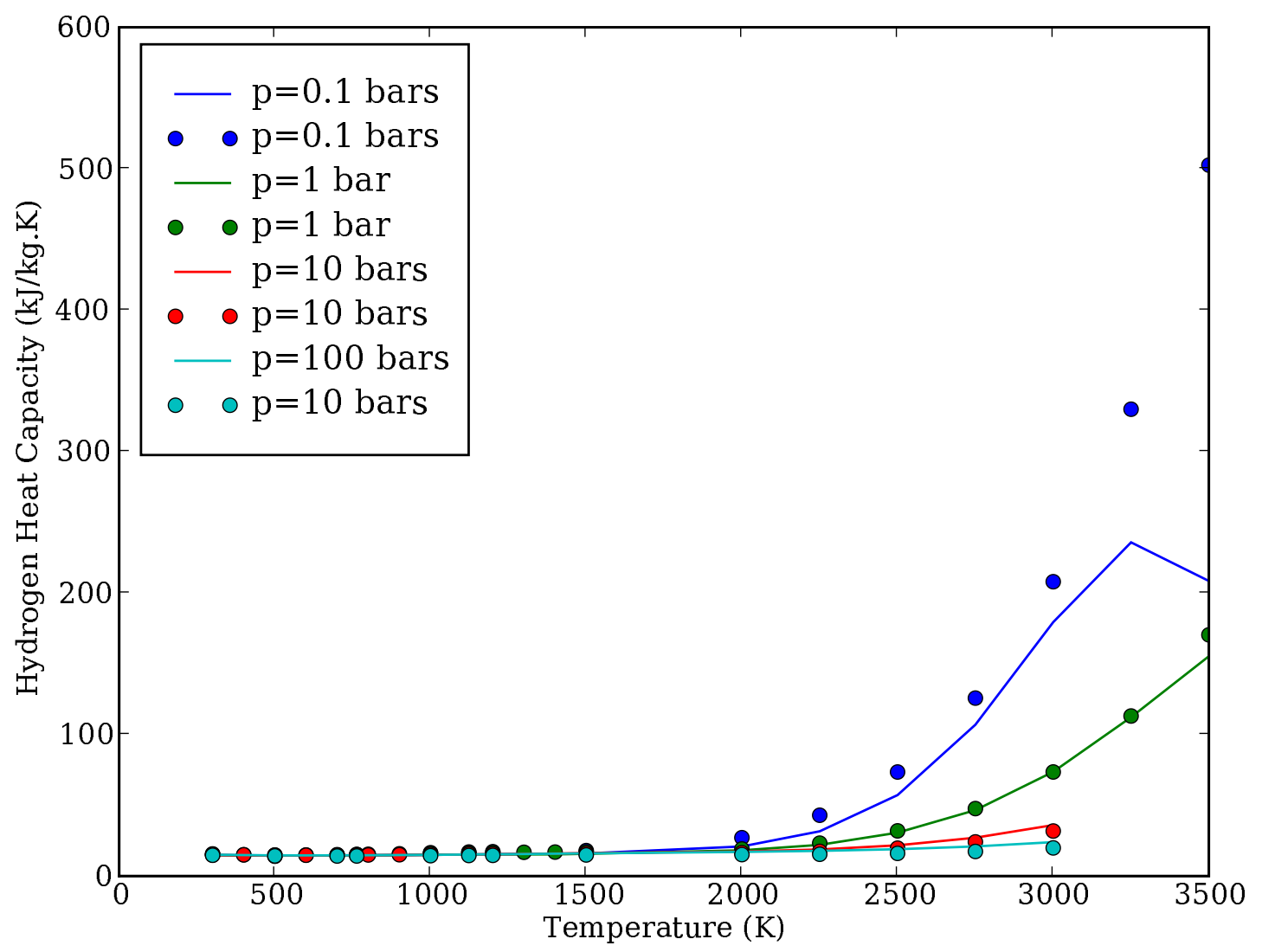

Figure 4-4: Hydrogen heat capacity vs. temperature: Comparison between experimental data (solid lines) and the model (dots).

Table 4-3: Error on the density formulation for $\mathrm{P}=10$ bar

\begin{tabular}{llllll}
\hline $\mathrm{P}(\mathrm{bar})$ & $\mathrm{T}(\mathrm{K})$ & $\mathrm{M}(\mathrm{g} / \mathrm{mol})$ & $\rho$ - IGL & $\rho$ - compiled & error $(\%)$ \\
\hline 10 & 1900 & 2.016 & $1.28 * 10^{-1}$ & $1.28 * 10^{-1}$ & 0.1 \\
10 & 2000 & 2.015 & $1.21 * 10^{-1}$ & $1.21 * 10^{-1}$ & 0.1 \\
10 & 2200 & 2.014 & $1.10 * 10^{-1}$ & $1.10 * 10^{-1}$ & 0.1 \\
10 & 2400 & 2.011 & $1.01 * 10^{-1}$ & $1.01 * 10^{-1}$ & 0.1 \\
10 & 2800 & 1.990 & $8.55 * 10^{-2}$ & $8.54 * 10^{-2}$ & 0.1 \\
10 & 3000 & 1.966 & $7.88 * 10^{-2}$ & $7.88 * 10^{-2}$ & 0.0 \\
10 & 3200 & 1.930 & $7.25 * 10^{-2}$ & $7.25 * 10^{-2}$ & 0.0 \\
10 & 3600 & 1.775 & $5.93 * 10^{-2}$ & $6.03 * 10^{-2}$ & 1.6 \\
\hline
\end{tabular}




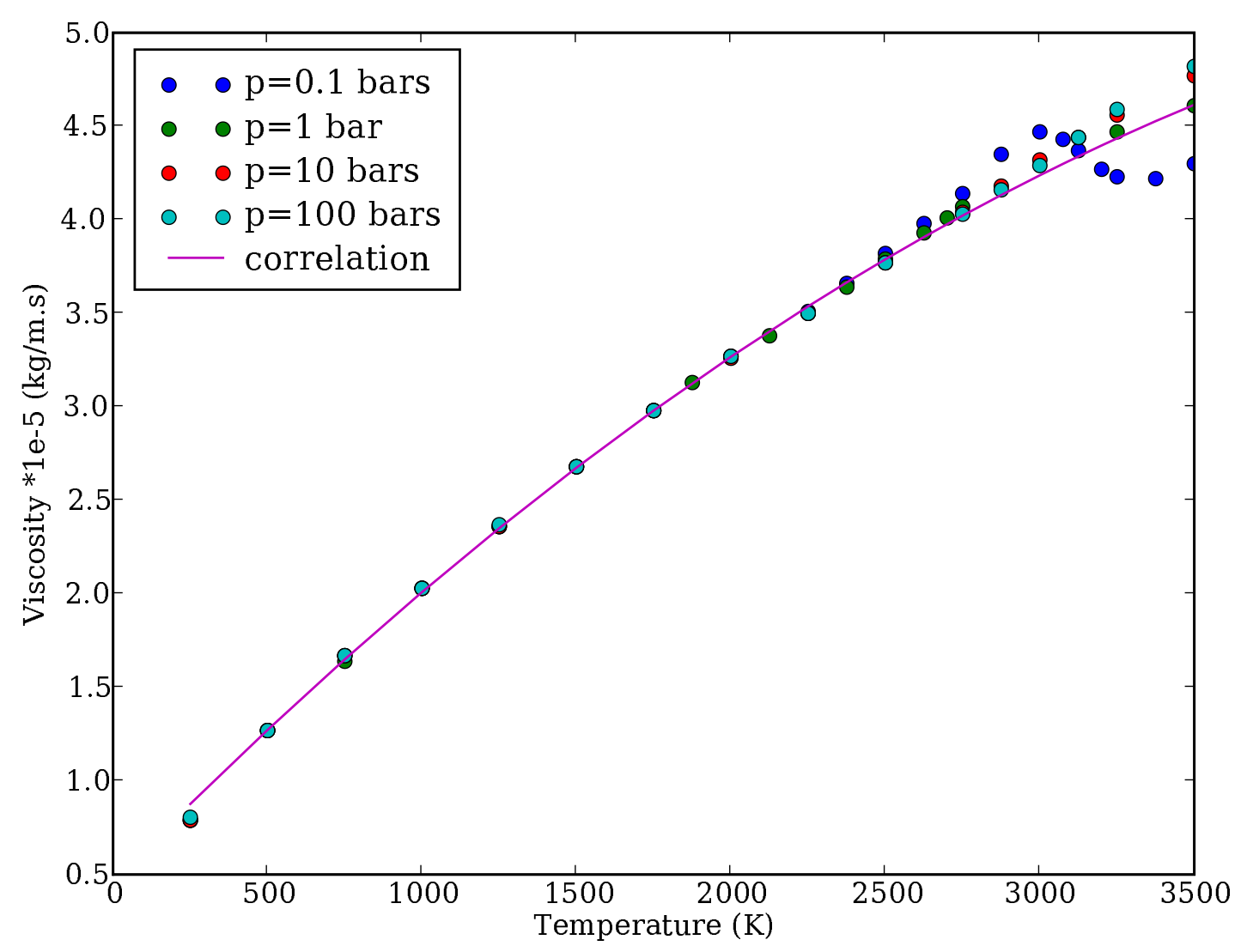

Figure 4-5: Hydrogen viscosity vs. temperature: Comparison between experimental data (dots) and the model (solid line).

Table 4-4: Error on the density formulation for $\mathrm{P}=100$ bar

\begin{tabular}{llllll}
\hline $\mathrm{P}(\mathrm{bar})$ & $\mathrm{T}(\mathrm{K})$ & $\mathrm{M}(\mathrm{g} / \mathrm{mol})$ & $\rho$ - IGL & $\rho$ - compiled & error $(\%)$ \\
\hline 100 & 2000 & 2.016 & 1.21 & 1.20 & 1.0 \\
100 & 2200 & 2.015 & 1.10 & 1.09 & 1.0 \\
100 & 2400 & 2.014 & 1.01 & 1.00 & 0.8 \\
100 & 2800 & 2.008 & $8.63 * 10^{-1}$ & $8.56 * 10^{-1}$ & 0.8 \\
100 & 3000 & 2.000 & $8.02 * 10^{-1}$ & $7.96 * 10^{-1}$ & 0.7 \\
100 & 3200 & 1.988 & $7.47 * 10^{-1}$ & $7.46 * 10^{-1}$ & 0.1 \\
100 & 3600 & 1.944 & $6.49 * 10^{-1}$ & $6.49 * 10^{-1}$ & 0.0 \\
\hline
\end{tabular}




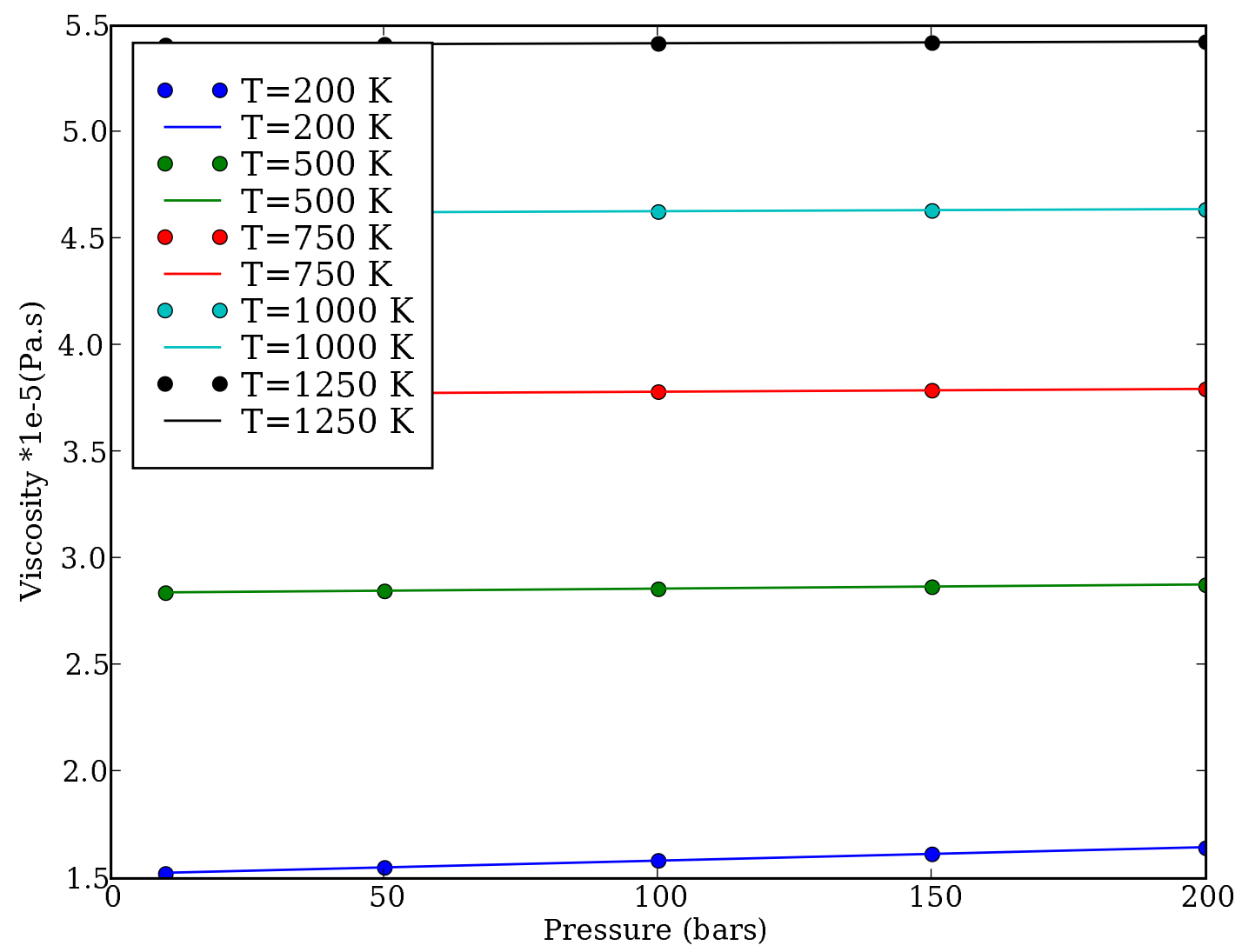

Figure 4-6: Helium viscosity vs. pressure for different temperatures: Comparison between experimental data (dots) and the model (solid line).

Table 4-5: Error on the density formulation for $\mathrm{P}=150$ bar

\begin{tabular}{llllll}
\hline $\mathrm{P}(\mathrm{bar})$ & $\mathrm{T}(\mathrm{K})$ & $\mathrm{M}(\mathrm{g} / \mathrm{mol})$ & $\rho$ - IGL & $\rho$ - compiled & error $(\%)$ \\
\hline 150 & 2200 & 2.016 & 1.65 & 1.63 & 1.5 \\
150 & 2400 & 2.014 & 1.51 & 1.50 & 1.3 \\
150 & 2800 & 2.010 & 1.30 & 1.28 & 1.2 \\
150 & 3000 & 2.003 & 1.20 & 1.19 & 1.0 \\
150 & 3200 & 1.993 & 1.12 & 1.12 & 0.1 \\
150 & 3600 & 1.957 & 0.98 & 0.98 & 0.1 \\
\hline
\end{tabular}




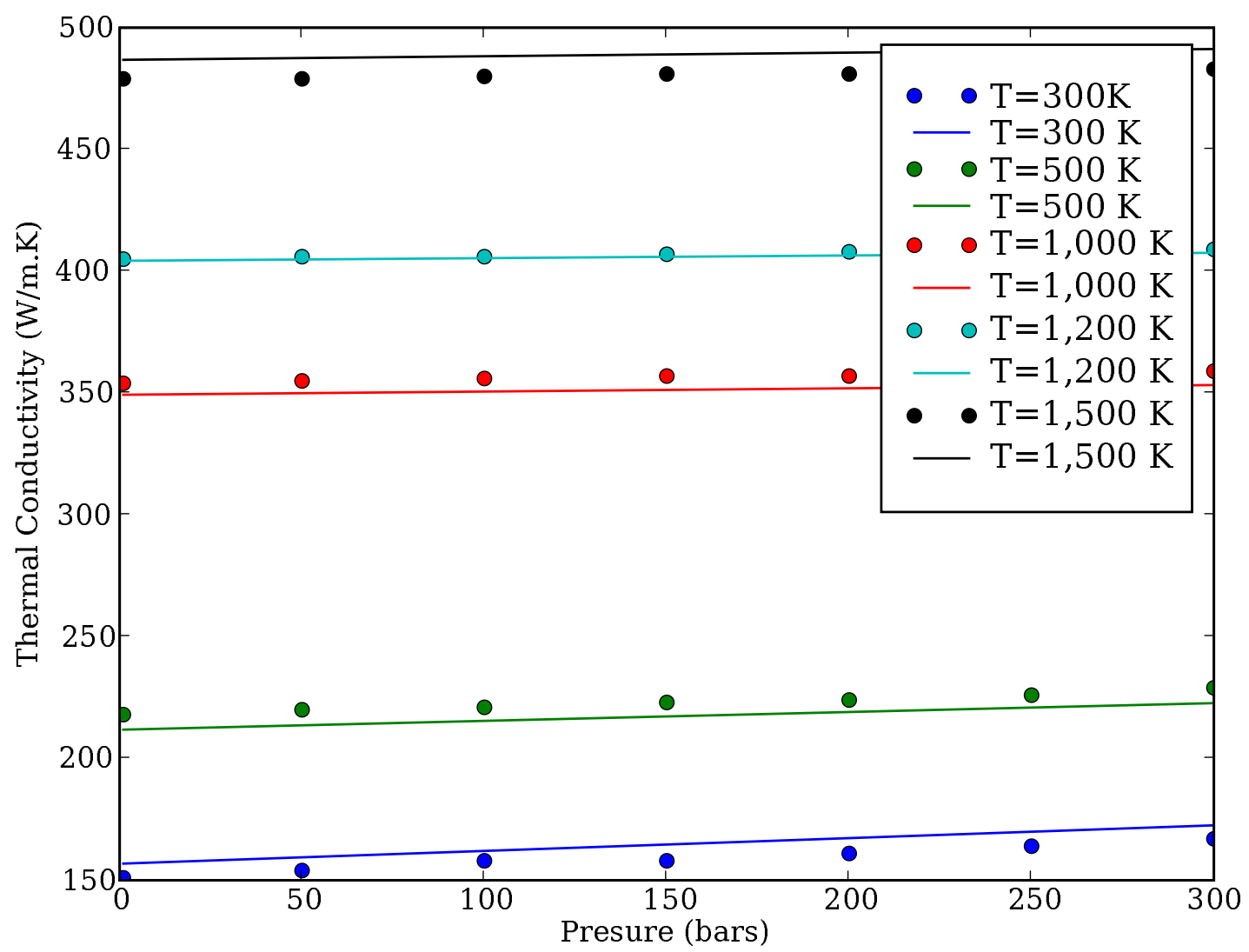

Figure 4-7: Helium thermal conductivity vs. pressure for different temperatures: Comparison between experimental data (dots) and the model (solid line).

Table 4-6: VHTR operating conditions and features

\begin{tabular}{ll}
\hline Condition or feature & VHTR \\
\hline power output $(\mathrm{MWt})$ & $600-900$ \\
average power density $\left(\mathrm{W} / \mathrm{cm}^{3}\right)$ & $4-6.5$ \\
coolant and pressure & helium @ $7.12 \mathrm{Mpa}$ \\
moderator & graphite \\
core geometry & annular \\
safety design philosophy & passive \\
plant design life & 60 years \\
core outlet temp. & $1,000^{\circ} \mathrm{C}$ \\
core inlet temp. & $490{ }^{\circ} \mathrm{C}$ \\
fuel - coated particles & a) LEU-PyC/SiC \\
& b) LEU-PyC $/ \mathrm{ZrC}$ \\
fuel max temp. & a) $\sim 1,250{ }^{\circ} \mathrm{C}$ \\
normal operation & b) $\sim 1,400{ }^{\circ} \mathrm{C}$ \\
\hline
\end{tabular}




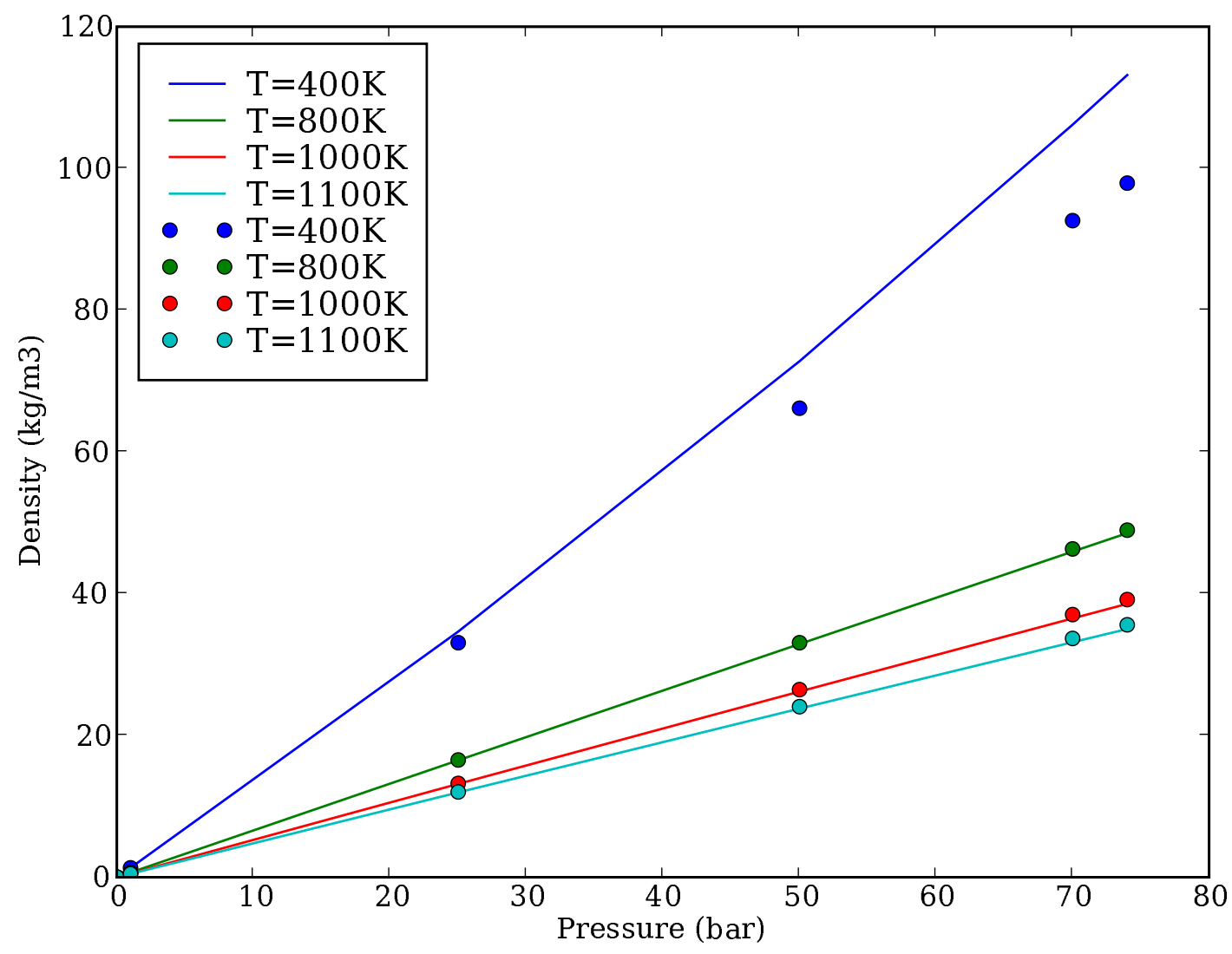

Figure 4-8: Carbon dioxide density vs. pressure for different temperatures. The solid lines represent the NIST data and the dots the ideal-gas law. 


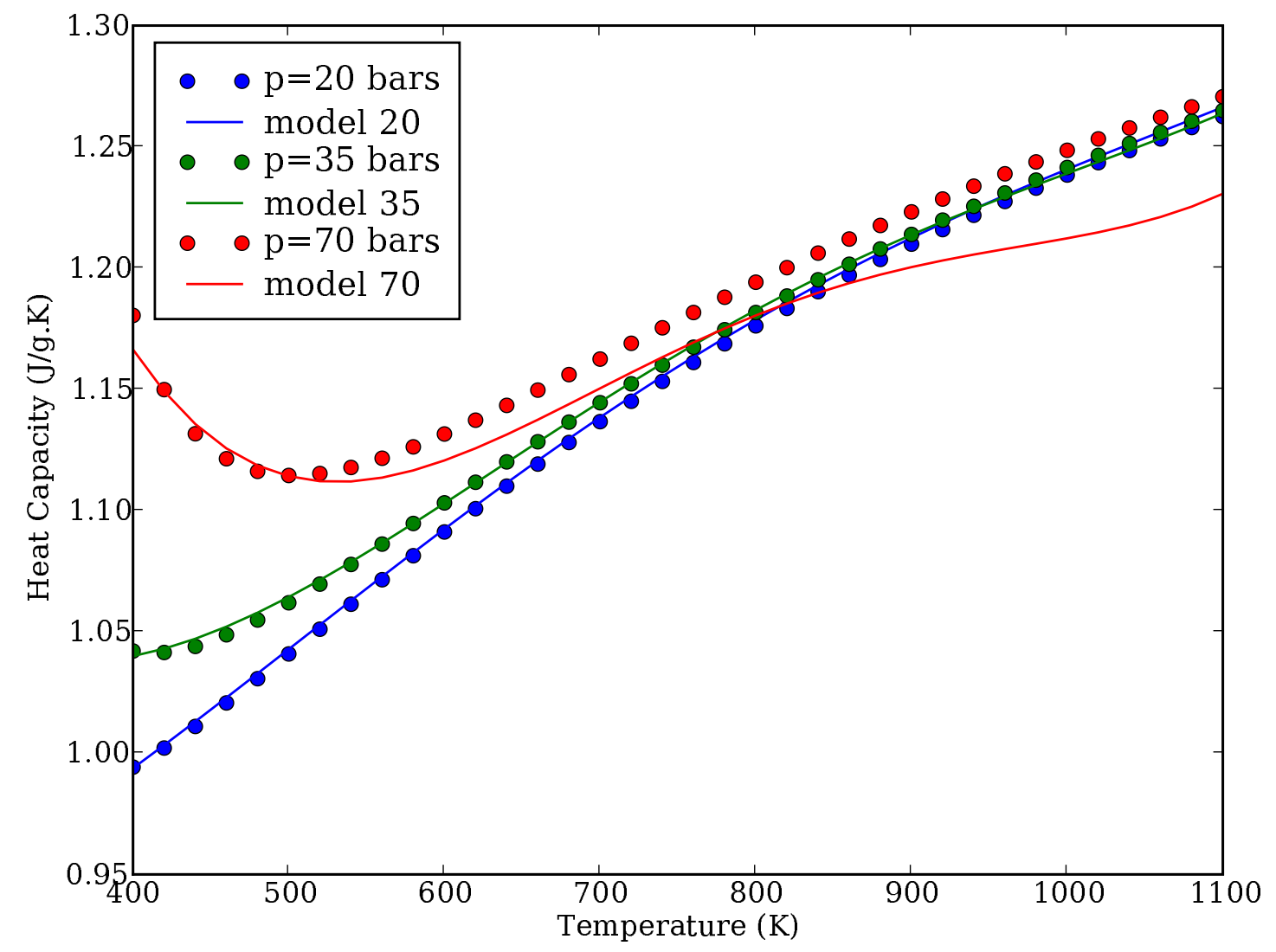

Figure 4-9: Carbon dioxide heat capacity vs. temperature for different pressures: Comparison between experimental data (dots) and the model (solid line). 


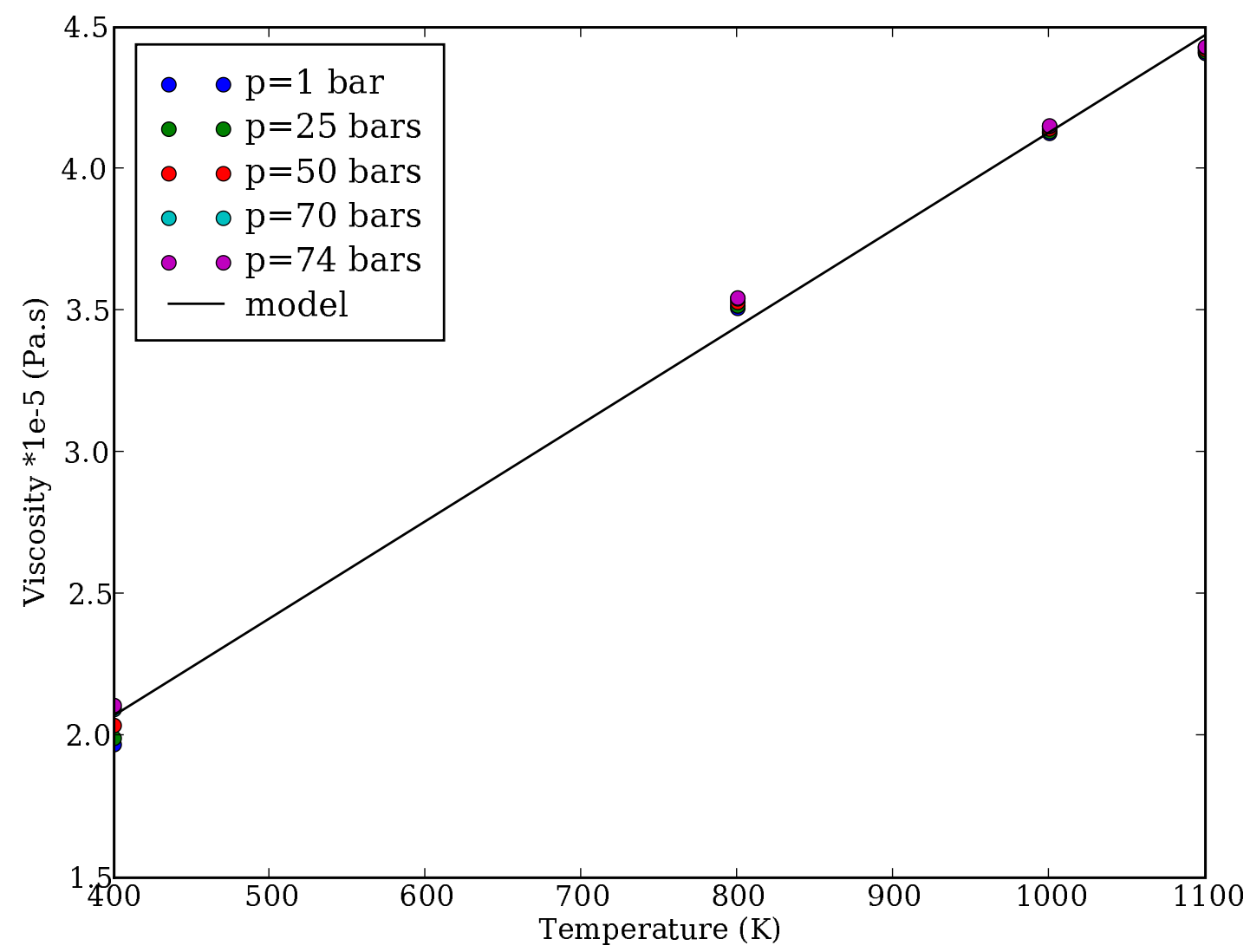

Figure 4-10: Carbon dioxide viscosity vs. temperature for different pressures: Comparison between experimental data (dots) and the model (solid line). 


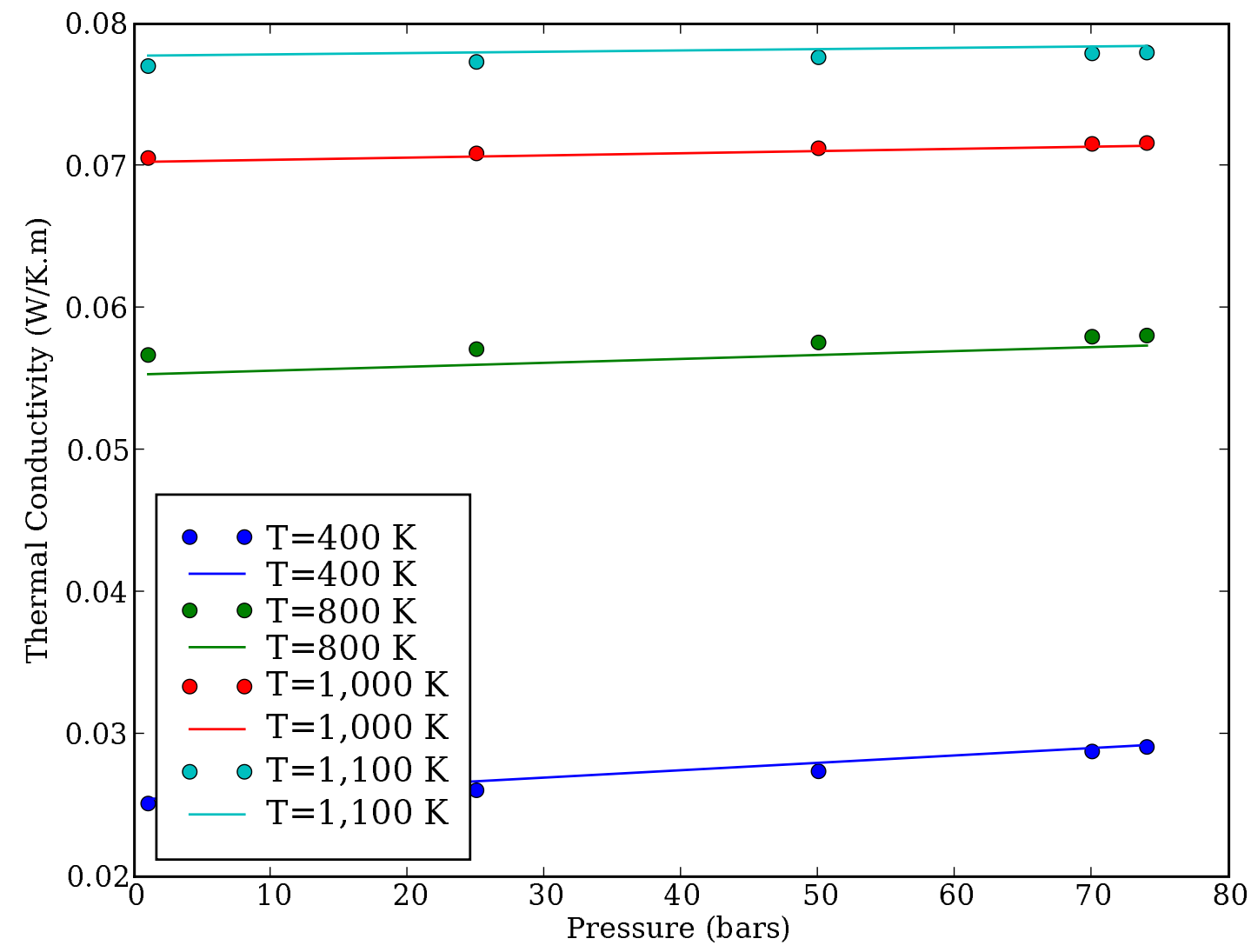

Figure 4-11: Carbon dioxide thermal conductivity vs. pressure for different temperatures: Comparison between experimental data (dots) and the model (solid line). 
Table 4-7: Comparison between experimental data and ideal-gas law for helium density

\begin{tabular}{lllll}
\hline P(bar $)$ & $\mathrm{T}\left({ }^{\circ} \mathrm{C}\right)$ & $\rho(\exp )$ & $\rho(\mathrm{IGL})$ & error $(\%)$ \\
\hline 1 & 200 & 0.10172 & 0.10176 & 0.04 \\
1 & 800 & 0.044855 & 0.04486 & 0.02 \\
10 & 200 & 1.01463 & 1.01757 & 0.29 \\
10 & 800 & 0.44814 & 0.44865 & 0.11 \\
40 & 200 & 4.025 & 4.07028 & 1.13 \\
40 & 800 & 1.7871 & 1.79458 & 0.42 \\
80 & 400 & 5.6387 & 5.72192 & 1.48 \\
80 & 800 & 3.5597 & 3.58916 & 0.83 \\
80 & 1000 & 3.0053 & 3.02534 & 0.67 \\
80 & 1400 & 2.2914 & 2.30207 & 0.47 \\
100 & 200 & 9.8996 & 10.17571 & 2.79 \\
100 & 800 & 4.4406 & 4.48645 & 1.03 \\
100 & 1000 & 3.7506 & 3.78167 & 0.83 \\
140 & 200 & 13.713 & 14.24599 & 3.89 \\
140 & 800 & 6.1919 & 6.28103 & 1.44 \\
140 & 1000 & 5.2339 & 5.29434 & 1.15 \\
\hline
\end{tabular}




\section{CHAPTER 5 \\ METHODS FOR THE CFD SOLVER DEVELOPMENT}

This chapter presents the methods we chose and we used to develop the real-property CFD solvers we use to analyze the diverse systems in Chapter 6 and 7 . We justify each choice of method based on our own experience and an extensive literature search. The final product is a combination of proven CFD techniques which are particularly well adapted to analyze gas flow in nuclear systems. The solver is validated on benchmark cases. Since we well-document our sources but do not develop our own numerical methods, we will not analyze consistency, stability, convergence, conservation and boundedness of our final solver. If the reader is interested in such an analysis, he is invited to consult the cited references.

\subsection{CFD Solver Development}

\subsubsection{Requirements}

The two systems we study with our CFD solver are the VHTR coolant channels and the NTP thrust chamber. Both systems exhibit an axial symmetry and allow a two-dimensional axisymmetric representation, as sketched Figure 5-1. The geometry of the system are relatively simple: the grids are composed of quadrilateral cells. Both flow system require a real-property analysis and are compressible. We are interested in the steady-state solution of such compressible and turbulent flows. First, we present the assumptions and derive the Reynolds Averaged Navier-Stokes equations. Then we present the time and space discretization techniques based on a Finite Volume method and a MacCormack implicit technique. We also give a description of the models which account for turbulence: $\mathrm{k}-\epsilon$ and Spalart-Allmaras. The two systems require similar boundary condition treatment: inlet velocity and temperature, exit pressure, heated walls and symmetry axis. 


\subsubsection{Governing Equations for Compressible Flow}

The governing equations for a compressible, viscous and turbulent flow are the set of Navier-Stokes equations. The local form of the equations is derived from the equations of conservation: conservation of mass, momentum and energy. The compact form of the system of equations is written [80]:

$$
\left\{\begin{array}{l}
\frac{\partial \rho}{\partial t}+\operatorname{div}(\rho \vec{V})=0 \\
\frac{\partial \rho \vec{V}}{\partial t}+\operatorname{div}(\rho \vec{V} \otimes \vec{V}+p \mathbf{I})=\operatorname{div}(\tau) \\
\frac{\partial E}{\partial t}+\operatorname{div}(H \vec{V})=\operatorname{div}(\tau \vec{V})-\operatorname{div}(\mathbf{s})
\end{array}\right.
$$

where, $t$ is the time, $\rho$ the density, $\vec{V}$ the velocity vector, $p$ the pressure, $\mathbf{I}$ the unity matrix, $\tau$ the shear stress tensor, $E$ the volumetric energy, $H$ the volumetric enthalpy and s the heat flux. The total volumetric energy is linked to the enthalpy by:

$$
H=E+p
$$

The heat source flux is defined by:

$$
\mathbf{s}=-\lambda(T) \nabla \vec{V}
$$

and $-\lambda(T)=\frac{c_{P} \mu}{P r}$ where, $c_{P}$ is the heat capacity at constant pressure, $\mu$ is the molecular viscosity and $\operatorname{Pr}$ is the Prandlt number.

The gases of considerations are hydrogen, helium and carbon dioxide: all three are Newtonian and the shear stress tensor in compact tensor notation becomes [38]:

$$
\tau_{i j}=\mu\left[\left(\frac{\partial u_{i}}{\partial x_{j}}+\frac{\partial u_{j}}{\partial x_{i}}\right)-\frac{2}{3} \delta_{i j} \frac{\partial u_{k}}{\partial x_{k}}\right]
$$

We derive the equations for an axisymmetric geometry: the polar coordinates are $(r, \theta, z)$ and the velocity components are $\vec{V}=\left(u_{r}, u_{\theta}, u_{z}\right)^{T}$. For an axisymmetric model, 
differentiation along $\theta$ is omitted. Because the flow is identical in any axial plane of the systems, the swirl velocity can be considered negligible, and hence $v_{\theta}=0$. The system of Navier-Stokes equations becomes [81]:

$$
\left\{\begin{array}{rlc}
\frac{\partial \rho}{\partial t}+\frac{\partial \rho u_{z}}{\partial z}+\frac{1}{r} \frac{\partial r \rho u_{r}}{\partial r} & = & 0 \\
\frac{\partial \rho u_{r}}{\partial t}+\frac{\partial \rho u_{z} u_{r}}{\partial z}+\frac{1}{r} \frac{\partial r \rho u_{r}^{2}}{\partial r} & = & -\frac{\partial p}{\partial r}+\frac{\partial \tau_{r z}}{\partial z}+\frac{1}{r} \frac{\partial r \tau_{r r}}{\partial r}+\frac{\tau_{\theta \theta}}{r}+\frac{\rho u_{\theta}^{2}}{r} \\
\frac{\partial \rho u_{z}}{\partial t}+\frac{\partial \rho u_{z}^{2}}{\partial z}+\frac{1}{r} \frac{\partial r \rho u_{r} u_{z}}{\partial r} & = & -\frac{\partial p}{\partial z}+\frac{\partial \tau_{z z}}{\partial z}+\frac{1}{r} \frac{\partial r \tau_{z r}}{\partial r}\left[\frac{c_{P} \mu}{P r} \frac{\partial T}{\partial z}+u_{z} \tau_{z z}+u_{r} \tau_{z r}+u_{\theta} \tau_{z \theta}\right] \\
\frac{\partial E}{\partial t}+\frac{\partial(E+p) u_{z}}{\partial z}+\frac{1}{r} \frac{\partial r(E+p) u_{r}}{\partial r} & +\frac{1}{r} \frac{\partial}{\partial r}\left[r\left(\frac{c_{P} \mu}{P r} \frac{\partial T}{\partial r}+u_{z} \tau_{r z}+u_{r} \tau_{r r}+u_{\theta} \tau_{r \theta}\right)\right]
\end{array}\right.
$$

and the tensor of shear stress $\tau$ is expressed by:

$$
\left[\begin{array}{ccc}
\tau_{z z} & \tau_{z r} & \tau_{z \theta} \\
\tau_{r z} & \tau_{r r} & \tau_{r \theta} \\
\tau_{\theta z} & \tau_{\theta r} & \tau_{\theta \theta}
\end{array}\right]=\mu\left[\begin{array}{ccc}
2 \frac{\partial u_{z}}{\partial z}-\frac{2}{3} \nabla \cdot \vec{V} & \frac{\partial u_{z}}{\partial r}+\frac{\partial u_{r}}{\partial z} & \frac{\partial u_{\theta}}{\partial z} \\
\frac{\partial u_{z}}{\partial r}+\frac{\partial u_{r}}{\partial z} & 2 \frac{\partial u_{r}}{\partial r}-\frac{2}{3} \nabla \cdot \vec{V} & \frac{\partial u_{\theta}}{\partial r} \\
\frac{\partial u_{\theta}}{\partial z} & \frac{\partial u_{\theta}}{\partial r} & -2 \frac{u_{r}}{r}-\frac{2}{3} \nabla \cdot \vec{V}
\end{array}\right]
$$

divergence of velocity vector becomes:

$$
\nabla \cdot \vec{V}=\frac{\partial u_{z}}{\partial z}+\frac{1}{r} \frac{\partial r u_{r}}{\partial r}=\frac{\partial u_{z}}{\partial z}+\frac{\partial u_{r}}{\partial r}+\frac{u_{r}}{r}
$$

The momentum equation in the $\theta$-direction is redundant and shall be ignored. We can write the previous set of equations in a compact form:

$$
\frac{\partial U}{\partial t}+\nabla \cdot F=\nabla \cdot F^{v}+S
$$


where

$$
\begin{aligned}
& U=\left[\begin{array}{c}
\rho \\
\rho u_{r} \\
\rho u_{z} \\
E
\end{array}\right] \quad, \quad F_{r}=\left[\begin{array}{c}
\rho u_{r} \\
\rho u_{r}^{2}+p \\
\rho u_{r} u_{z} \\
u_{r}(E+p)
\end{array}\right] \quad, \quad F_{z}=\left[\begin{array}{c}
\rho u_{z} \\
\rho u_{r} u_{z} \\
\rho u_{z}^{2}+p \\
u_{z}(E+p)
\end{array}\right] \\
& F_{r}^{v}=\left[\begin{array}{c}
0 \\
\tau_{r r} \\
\tau_{r z} \\
\frac{c_{P} \mu_{t o t}}{P r} \frac{\partial T}{\partial r}+u_{r} \tau_{r r}+u_{z} \tau_{r z}+u_{\theta} \tau_{r \theta}
\end{array}\right] \quad, F_{z}^{v}=\left[\begin{array}{c}
0 \\
\tau_{z r} \\
\tau_{z z} \\
\frac{c_{P} \mu_{t o t}}{P r} \frac{\partial T}{\partial z}+u_{r} \tau_{z r}+u_{z} \tau_{z z}+u_{\theta} \tau_{z \theta}
\end{array}\right] \\
& S=\left[\begin{array}{c}
0 \\
0 \\
-\frac{\tau_{\theta \theta}}{r} \\
0
\end{array}\right]
\end{aligned}
$$

\subsubsection{Need for the Equation of State}

The system of Equations 5-1 is composed of four equations. There are five unknowns: $\rho, u_{r}, u_{z}, E$ and $p$. Therefore an equation of state is required to relate the density to the thermodynamic variables. In the previous chapter we determined that the ideal gas law describes properly density variations with temperature and pressure for all three gases of consideration. The equation of state for our solver will be:

$$
p=\rho R T
$$

where $R$ is the universal gas constant. We then have the extra equation linking energy, pressure and velocity:

$$
E=\frac{p}{\gamma-1}+\frac{1}{2}\|\vec{V}\|^{2}
$$




\subsubsection{Reynolds-Averaged Navier-Stokes Equations}

The system of governing equations 5-1 only holds for laminar flows. In Reynolds-averaged approaches to turbulence, all the unsteadiness is averaged out. In a statistically steady flow, every variable $\phi\left(x_{i}, t\right)$ can be written as the sum of a time averaged value (mean flux) and fluctuation about that value (fluctuating flux):

$$
\phi\left(x_{i}, t\right)=\bar{\phi}\left(x_{i}\right)+\phi^{\prime}\left(x_{i}, t\right)
$$

where

$$
\bar{\phi}\left(x_{i}\right)=\lim _{T \rightarrow+\infty} \frac{1}{T} \int_{0}^{T} \phi\left(x_{i}, t\right) d t
$$

If $T$ is large enough, $\bar{\phi}$ does not depend on the time at which the averaging started. From equation $5-11$, it follows that $\overline{\phi^{\prime}}=0$. Reynolds average is better suited for incompressible flows ( $\rho=$ constant). Compressible flows introduce extra fluctuations, in particular fluctuations in density $\rho$, which add extra correlation terms. In the situation of compressible flow, Favre-averaging is used for $u_{i}, \tau_{i j}, T, h, q_{j} \ldots$ and Reynolds-averaging is used for $\rho$ and $p$. Favre-averaging (represented by $\langle$.$\rangle ) is obtained by mass-averaging:$

$$
q=\langle q\rangle+q^{\prime \prime} \quad\langle q\rangle=\frac{\overline{\rho q}}{\bar{\rho}}
$$

Favre-averaging obeys the following rules:

$$
\overline{\rho q^{\prime \prime}}=0 \quad \overline{\rho q}=\bar{\rho}\langle q\rangle \quad \overline{\rho q r}=\bar{\rho}\langle q\rangle\langle r\rangle+\overline{\rho q^{\prime \prime} r^{\prime \prime}}
$$

We now apply these calculations rules to derive the RANS equation in polar coordinates, for axisymmetric geometry. We use the assumption that the mean density can be written as $\rho$ and that the swirl velocity is equal to zero. However, its fluctuations $u_{\theta}^{\prime \prime}$ need to be accounted for. We neglect the mass-average of the shear stress fluctuations over the mass-average of the shear stress: $\overline{\tau_{i j}^{\prime \prime}} \ll\left\langle\tau_{i j}\right\rangle$. We also assume that interactions between viscous shear stress and turbulent mass flux are neglected. Thus, $\overline{u_{i} \tau_{i j}}=$ 
$\left\langle u_{i}\right\rangle\left\langle\tau_{i j}\right\rangle+\overline{u_{i}^{\prime \prime} \tau_{i j}}+\left\langle u_{i}\right\rangle \overline{\tau_{i j}^{\prime \prime}}$. After mass-averaging, the mean-flow conservation equations in 2-D axisymmetric becomes [81]:

$$
\begin{aligned}
& \int \frac{\partial \rho}{\partial t}+\frac{\partial \rho\left\langle u_{r}\right\rangle}{\partial r}+\frac{\partial \rho\left\langle u_{z}\right\rangle}{\partial z} \quad=-\frac{\rho\left\langle u_{r}\right\rangle}{r} \\
& \frac{\partial \rho\left\langle u_{r}\right\rangle}{\partial t}+\frac{\partial \rho\left\langle u_{r}\right\rangle^{2}}{\partial r}+\frac{\partial \rho\left\langle u_{r}\right\rangle\left\langle u_{z}\right\rangle}{\partial z}=-\frac{\partial \bar{p}}{\partial r}+\frac{\partial\left\langle\tau_{r r}\right\rangle}{\partial r}+\frac{\partial\left\langle\tau_{r z}\right\rangle}{\partial z}-\frac{1}{r}\left(\rho\left(\left\langle u_{r}\right\rangle^{2}-\left\langle u_{\theta}\right\rangle^{2}\right)\right) \\
& +\frac{\left\langle\tau_{r r}\right\rangle}{r}+\frac{\left\langle\tau_{\theta \theta}\right\rangle}{r}-\frac{\partial \overline{\rho u_{r}^{\prime \prime} u_{r}^{\prime \prime}}}{\partial r}-\frac{\partial \overline{\rho u_{r}^{\prime \prime} u_{z}^{\prime \prime}}}{\partial z} \\
& -\frac{1}{r}\left(\overline{\rho u_{r}^{\prime \prime} u_{r}^{\prime \prime}}-\overline{\rho u_{\theta}^{\prime \prime} u_{\theta}^{\prime \prime}}\right) \\
& \frac{\partial \rho\left\langle u_{z}\right\rangle}{\partial t}+\frac{\partial \rho\left\langle u_{r}\right\rangle\left\langle u_{z}\right\rangle}{\partial r}+\frac{\partial \rho\left\langle u_{z}\right\rangle\left\langle u_{z}\right\rangle}{\partial z}=-\frac{\partial \bar{p}}{\partial z}+\frac{\partial\left\langle\tau_{r z}\right\rangle}{\partial \underline{r}}+\frac{\partial\left\langle\tau_{z z}\right\rangle}{\partial z}-\frac{1}{r} \rho\left\langle u_{r}\right\rangle\left\langle u_{z}\right\rangle \\
& +\frac{1}{r}\left\langle\tau_{r z}\right\rangle-\frac{\partial \overline{\rho u_{r}^{\prime \prime} u_{z}^{\prime \prime}}}{\partial r}-\frac{\partial \overline{\rho u_{z}^{\prime \prime} u_{z}^{\prime \prime}}}{\partial z}-\frac{1}{r} \overline{\rho u_{r}^{\prime \prime} u_{z}^{\prime \prime}} \\
& \frac{\partial\langle E\rangle}{\partial t}+\frac{\partial\left(\left\langle u_{r}\right\rangle(\langle E\rangle+\bar{p})\right)}{\partial r} \quad+\frac{\partial\left(\left\langle u_{z}\right\rangle(\langle E\rangle+\bar{p})\right)}{\partial z}=-\frac{1}{r}\left(\left\langle u_{r}\right\rangle(\langle E\rangle+\bar{p})\right) \\
& +\frac{\partial}{\partial z}\left[\left\langle u_{z}\right\rangle\left(\left\langle\tau_{z z}\right\rangle-\overline{\rho u_{z}^{\prime \prime} u_{z}^{\prime \prime}}\right)+\left\langle u_{r}\right\rangle\left(\left\langle\tau_{r z}\right\rangle-\overline{\rho u_{r}^{\prime \prime} u_{z}^{\prime \prime}}\right)\right] \\
& +\frac{\partial}{\partial z}\left[\left(\frac{\mu}{P r}+\frac{\mu_{t}}{P r_{t}}\right) c_{P} \frac{\partial\langle T\rangle}{\partial z}\right] \\
& +\frac{1}{r} \frac{\partial}{\partial r}\left[r\left(\left\langle u_{z}\right\rangle\left(\left\langle\tau_{r z}\right\rangle-\overline{\rho u_{r}^{\prime \prime} u_{z}^{\prime \prime}}\right)+\left\langle u_{r}\right\rangle\left(\left\langle\tau_{r r}\right\rangle-\overline{\rho u_{r}^{\prime \prime} u_{r}^{\prime \prime}}\right)\right)\right] \\
& +\frac{1}{r} \frac{\partial}{\partial r}\left[r\left(\frac{\mu}{P r}+\frac{\mu_{t}}{P r_{t}}\right) c_{P} \frac{\partial\langle T\rangle}{\partial r}\right]
\end{aligned}
$$

Equations 5-13 are the system of Reynolds Averaged Navier-Stokes (RANS) equations.

\subsubsection{Closure Problem: the Need for Turbulence Model}

Derivation of the time-averaged equations introduce four unknowns in the time-averaged momentum equations: namely, the turbulent stresses $-\overline{\rho u_{i}^{\prime \prime} u_{j}^{\prime \prime}}$. In order to obtain closure of the system of equations, the turbulent stresses need to be modeled. The double correlation between $u_{i}^{\prime \prime}$ and itself is the kinetic energy per unit volume $(k)$ of the turbulent velocity 
fluctuations. Thus, we define:

$$
\bar{\rho} k=\frac{1}{2} \overline{\rho u_{i}^{\prime \prime} u_{i}^{\prime \prime}}
$$

The terms $\overline{\rho u_{i}^{\prime \prime} u_{j}^{\prime \prime}}$ are called the Reynolds-stress tensor and are denoted by:

$$
\bar{\rho} \sigma_{i j}=-\overline{\rho u_{i}^{\prime \prime} u_{j}^{\prime \prime}}
$$

The approach to model the turbulent stresses defines the type of turbulence model chosen. There are a lot of turbulence models which have been developed since the early 70s. Each one has its particular domain of application, computational requirements. We classify and present the main models of Reynolds Averaged Navier Stokes turbulence.

\subsubsection{Turbulence models: classification and overview}

RANS models can be divided into two broad approaches: the first category is based on the Boussinesq hypothesis, the second one on the Reynolds Stress Models.

1. The Boussinesq hypothesis relates the Reynolds stresses to the mean velocity gradients:

$$
-\overline{\rho u_{i} \prime \prime u_{j} \prime \prime}=\mu_{t}\left(\frac{\partial u_{i}}{\partial x_{j}}+\frac{\partial u_{j}}{\partial x_{i}}\right)-\frac{2}{3}\left(\rho k+\mu_{t} \frac{\partial u_{i}}{\partial x_{i}}\right) \delta_{i j}
$$

This method involves using an algebraic equation for the Reynolds stresses which include determining the turbulent viscosity, and depending on the level of sophistication of the model, solving transport equations for determining the turbulent kinetic energy, $k$, and dissipation, $\epsilon$. The advantage of this approach is the relatively low computational cost associated with the computation of the turbulent viscosity $\mu_{t}$. The models available in this approach are often referred to by the number of transport equations:

(a) Zero-equation: In a zero-equation models or algebraic models no transport equations are solved. The models are calculated directly from flow properties. The two most common algebraic models are the Baldwin-Lomax [63] and Cebeci-Smith models [82] which are based on:

$$
\mu_{t}= \begin{cases}\mu_{\text {inner }} & \text { if } z \leq z_{b} \\ \mu_{\text {outer }} & \text { if } z>z_{b}\end{cases}
$$

where $z$ is the normal distance from the wall, and $z_{b}$ is the smallest value of $z$ at which values from inner and outer formulae are equal. The formulation of $\mu_{\text {inner }}$ and $\mu_{\text {outer }}$ depends of the model of choice. The algebraic models are often too simple for use in general situations, but can be quite useful for simpler flow 
geometries, in start-up situations and they are quite accurate for high-speed flows with thin attached boundary layers.

(b) One-equation: One-equation models solve one turbulent transport equation, usually the turbulent kinetic energy k. Historically, the first model was the Prandtl's one-equation model [83]. In 1945, Prandtl introduced the notion that differential equations must be introduced to overcome the limitations of algebraic models [84]. The equations should have both convective (first-order) and diffusive (second-order) terms to explain how turbulence created at one point could have an effect somewhere else. However, Prandtl introduced only one such equation: his model therefore had the disadvantage that the length scale still had to be guessed. The differential transport equation to solve for is:

$$
\frac{\partial k}{\partial t}+U_{j} \frac{\partial k}{\partial x_{j}}=\tau_{i j} \frac{\partial U_{i}}{\partial x_{j}}-C_{D} \frac{k^{3 / 2}}{l}+\frac{\partial}{\partial x_{j}}\left[\left(\nu+\frac{\nu_{t}}{\sigma_{k}}\right) \frac{\partial k}{\partial x_{j}}\right]
$$

where $C_{D}$ and $\sigma_{k}$ are constants and $\nu_{t}=k^{1 / 2} l$.

Another one-equation, which use is growing particularly in the area of supersonic wall-bounded flows, is the Spalart-Allmaras model [69]. Detailed description of this model is given in the following section.

(c) Two-equation: Two-equation models are the RANS models which are most commonly used in turbulence modeling for CFD techniques. Models like the $\mathrm{k}-\epsilon$ model [85] and the $\mathrm{k}-\omega$ [86] model have become industry standard models and are commonly used for most types of engineering problems. Two equation turbulence models are also very much still an active area of research and new refined two-equation models are still being developed.

2. The Reynolds Stress Models (RSM) attempts to actually solve transport equations for the Reynolds stresses. This means introduction of several transport equations for all the Reynolds stresses and hence this approach is much more costly in CPU effort. For the cases we study in the scope of this doctoral research RSM models do not bring a lot more accuracy, but lengthen significantly the computational time.

\subsubsection{2 k- $\epsilon$ model}

$\mathrm{K}-\epsilon$ model is a two-equation level of closure which attempt to develop transport equations for both the turbulent velocity and length scales of the flow [70]. The k- $\epsilon$ is one attempt to solve for the transport equations, and uses the turbulent kinetic energy for the turbulent velocity scale equation and the turbulent energy dissipation rate for the length 
scale equation. The transport equation in their differential form are [65]:

$$
\begin{gathered}
\frac{\partial(\rho k)}{\partial t}+\operatorname{div}(\rho k \vec{V})=\operatorname{div}\left[\frac{\mu_{t}}{\sigma_{k}} \operatorname{gradk}\right]+2 \mu_{t} \tilde{E}_{i, j} \cdot \tilde{E}_{i, j}-\rho \epsilon \\
\frac{\partial \rho \epsilon}{\partial t}+\operatorname{div}(\rho \epsilon \vec{V})=\operatorname{div}\left[\frac{\mu_{t}}{\sigma_{\epsilon}} \operatorname{grad}\right]+C_{1 \epsilon} \frac{\epsilon}{k} 2 \mu_{t} \tilde{E}_{i, j} \cdot \tilde{E}_{i, j}-C_{2 \epsilon} \rho \frac{\epsilon^{2}}{k}
\end{gathered}
$$

where,

$$
\mu_{t}=\rho C_{\mu} \frac{k^{2}}{\epsilon}
$$

and $\tilde{E}$ is the mean rate of deformation of a fluid element [65].

$$
\tilde{E}_{i, j} \cdot \tilde{E}_{i, j} \approx \frac{1}{2}\left(\frac{\partial\left\langle u_{i}\right\rangle}{\partial x_{j}}+\frac{\partial\left\langle u_{j}\right\rangle}{\partial x_{i}}\right) \frac{\partial\left\langle u_{i}\right\rangle}{\partial x_{j}}
$$

Equations 5-18, 5-19 and 5-20 contain five adjustable constants. We will consider the standard $\mathrm{k}-\epsilon$ model for which:

$$
\left\{\begin{array}{c}
C_{\mu}=0.09 \\
\sigma_{k}=1.00 \\
\sigma_{\epsilon}=1.30 \\
C_{1 \epsilon}=1.44 \\
C_{2 \epsilon}=1.92
\end{array}\right.
$$

\subsubsection{The Spalart-Allmaras model}

In the thrust chamber study hydrogen flow is wall-bounded: the flow is subsonic at the inlet and as the gas expands in the converging-diverging nozzle the flow becomes supersonic. The Spalart-Allmaras turbulence model was created and developed for analysis of this particular type of flow [69]: it is a one-equation model based primarily on empiricism and on dimensional analysis arguments [70]. First publication of the model dates back to 1992. Although it is a quite recent model, it has been the object of numerous validations and shows great efficiency in prediction of wall-bounded flows with a reduced computational time expense. For example, Paciorri [87] validated the 
model for boundary layers in the flow on a plate with Mach numbers of 0.3, 2.0 and 6.0. It shows that for flows with no abrupt geometrical transitions the Spalart-Allmaras turbulence model gives satisfactory results. Lorin et al. [88] also prove the quality of the flow predictions on the flat plate benchmark exercise when they use the Spalart-Allmaras model, as long as the positivity of the turbulent viscosity is preserved. This model is local, i.e. the equation a one point does not depend on the solution at other points: the model is easily usable on all types of grids. Therefore this model is easy to implement and is fast.

In the Spalart-Allmaras model, the eddy or apparent turbulent viscosity $\mu_{t}$ is computed through a partial differential equation. The intermediate value $\tilde{\nu}$ follows the relation:

$$
\nu_{t}=\frac{\mu_{t}}{\rho}=\tilde{\nu} f_{v 1}(\chi),
$$

where $\chi$ is the ratio

$$
\chi=\frac{\tilde{\nu}}{\nu}
$$

and $f_{v 1}$ is a damping function:

$$
f_{v 1}=\frac{\chi^{3}}{\chi^{3}+c_{v 1}^{3}}
$$

The variable $\tilde{\nu}$ is obtained through the transport equation:

$$
\begin{array}{r}
\frac{D \tilde{\nu}}{D t}=c_{b 1}\left[1-f_{t 2}\right] \tilde{S} \tilde{\nu}+\frac{1}{\sigma}\left[\nabla \cdot((\nu+\tilde{\nu}) \nabla \tilde{\nu})+c_{b 2}(\nabla \tilde{\nu})^{2}\right]- \\
{\left[c_{w 1} f_{w}-\frac{c_{b 1}}{\kappa^{2}} f_{t 2}\right]\left[\frac{\tilde{\nu}}{d}\right]^{2}+f_{t 1} \Delta U^{2}}
\end{array}
$$

The four terms of the right hand of the equation are the production term, the diffusion term, the destruction term and the source term, respectively. For the production term, the vorticity magnitude $S$ is replaced by $\tilde{S}$ defined as:

$$
\tilde{S} \equiv S+\frac{\nu}{\kappa^{2} d^{2}} f_{\nu 2}
$$

where the function $f_{\nu 2}$ is

$$
f_{\nu 2}=1-\frac{\chi}{1+\chi f_{\nu 1}}
$$


and $d$ is the distance to the closest wall. The function $f_{t 2}$ is simply used to ensure that $\nabla \nu$ is a stable solution:

$$
f_{t 2}=c_{t 3} e^{-c_{t 4} \chi^{2}}
$$

No particular function appears in the diffusion term; for the destruction term of the function

$$
f_{w}(r)=g\left[\frac{1+c_{w 3}^{6}}{g^{6}+c_{w 3}^{6}}\right]^{4} \quad g=r+c_{w 2}\left(r^{6}-r\right) \quad r \equiv \frac{\mu_{t}}{S \kappa^{2} d^{2}}
$$

Finally, the trip term or source term $\Delta U$ represents the velocity difference from the field to the trip point. The function $f_{t 1}$ is computed as follows:

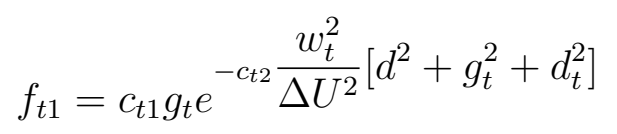

where $w_{t}$ is the wall vorticity at the trip point and $d_{t}$ the distance from the field to the trip point. The function $g_{t}$ writes:

$$
g_{t} \equiv \min \left(0.1, \Delta x_{t} \frac{\Delta U}{w_{t}}\right)
$$

As a starting point the default numbers given in [69] are used:

$$
\begin{array}{ccc}
c_{b 1}=0.1355 & \sigma=2 / 3 & c_{b 2}=0.633 \\
\kappa=0.41 & c_{w 1}=2.8 & c_{w 2}=0.3 \\
c_{w 3}=2 & c_{v 1}=7.1 & c_{t 1}=1 \\
c_{t 2}=2 & c_{t 3}=1.2 & c_{t 4}=0.5
\end{array}
$$

\subsubsection{Space Discretization}

Using the fact that we only consider time-averaged quantities, we can rewrite the Reynolds-averaged equations in the compact form, omitting the $<>$ notation with Equation 5-8: where we now have

$$
\tau_{i j}=\mu_{t o t}\left[\left(\frac{\partial u_{i}}{\partial x_{j}}+\frac{\partial u_{j}}{\partial x_{i}}\right)-\frac{2}{3} \delta_{i j} \frac{\partial u_{k}}{\partial x_{k}}\right]
$$


and $\mu_{t o t}=\mu+\mu_{t}$. All the variables are time-averaged following Favre or Reynolds-averaging as described above. The method to derive $\mu_{t}$ depends on the RANS turbulence model chosen:

$$
\begin{cases}\mu_{t}=\rho \tilde{\nu} f_{\nu 1}(\chi) & \text { Spalart-Allmaras } \\ \mu_{t}=\rho C_{\mu} \frac{k^{2}}{\epsilon} & \mathrm{k}-\epsilon\end{cases}
$$

First, we use a Navier-Stokes two-dimensional conservative solver. Then we add corrections to account for axisymmetric behavior and finally we present the algorithm to solve the turbulent model [89].

Discretization methods are used to convert the set of governing partial differential equations 5-8 and turbulent transport equations into algebraic equations at each grid node. It is the heart of numerical methods and particular attention is focuses on discretization methods research: The equivalent algebraic relations should faithfully represent the original PDEs. Each differential term is transformed into an approximate algebraic relation particularly adapted to the nature of the term. Finite Difference Method (FDM), Finite Volume Method (FVM) and Finite Elements Method (FEM) are the most used and developed methods in the solution of partial differential equations for numerical methods in general and for CFD in particular. In a FDM, the derivatives are approximated at the selected grid points using Taylor series expansion. More details are given in [64]. FVM is based on the control volume formulation of analytical fluid dynamics. The domain is divided into small control volumes and the variable is located at the centroid of the control volumes. Approximations are made on the surface and volume integrals. The FEM belongs to the class of weighted residual methods: The transport variable $\phi$ is approximated by a polynomial function $\bar{\phi}=a_{0}+a_{1} x+a_{2} x^{2}+\ldots+a_{m} x^{m}$. By choosing a succession of weight functions, one can generate as many equations as there are unknowns $\left(a_{i}\right)$ thus yielding a algebraic system of equations.

The most compelling feature of the FVM is that the resulting solution satisfies the conservation of quantities such as mass, momentum, energy, and species. This is exactly 
satisfied for any control volume as well as for the whole computational domain and for any number of control volumes. Even a coarse grid solution exhibits exact integral balances and FVM is the ideal method for computing discontinuous solutions arising in compressible flows. Therefore it is the most commonly used method in engineering numerical method studies. Hence, techniques, applications, limitations and advancements are well documented. Naturally, this is the method we choose to develop our CFD solvers.

The Finite Volume Method

The key feature of the FVM is that the governing equations in their integral form are integrated over a control volume, in general the cell volume. Control points are located at the centroid of the volume, midway between successive node points. Location of the numerical nodes in a quadrilateral 2-D domain composed of 5x5 cells are given Figure 5-2. The derivation of the algebraic governing equations for each computational cell requires the use of Gauss' theorem or divergence theorem:

$$
\iiint \nabla \cdot F d V=\oiint F \cdot d \vec{S}
$$

Figure 5-3 shows the infinitesimal volume expressed in cylindrical coordinates. We get $d V=r d r d \theta d z$ and

$$
d \vec{S}=r d \theta d z \overrightarrow{e_{r}}+d r d z \overrightarrow{e_{\theta}}+r d r d \theta \overrightarrow{e_{z}}
$$

In the case where we apply the FVM to the divergence of $F$ on the control volume/cell $(i, j)$. Using the notations from Figure 5-4 we get:

$$
\begin{aligned}
\iiint \nabla \cdot F r d r d \theta d z & =\oiint F \cdot d \vec{S} \\
& =\iint F_{r}^{+} r d z d \theta+\iint \overbrace{F_{\theta}^{+}}^{0} d r d z+\iint F_{z}^{+} r d r d \theta-F_{r}^{-} r d z d \theta-\ldots \\
& =2 \pi\left[\int_{1}\left(F_{r}\left(i+\frac{1}{2}, j\right) r d z+F_{z}\left(i+\frac{1}{2}, j\right) r d r\right)+\int_{2}\left(F_{r}\left(i, j+\frac{1}{2}\right) r d z+F_{z}\left(i, j+\frac{1}{2}\right) r d r\right)\right. \\
& \left.-\int_{3}\left(F_{r}\left(i-\frac{1}{2}, j\right) r d z+F_{z}\left(i-\frac{1}{2}, j\right) r d r\right)-\int_{4}\left(F_{r}\left(i, j-\frac{1}{2}\right) r d z+F_{z}\left(i, j-\frac{1}{2}\right) r d r\right)\right]
\end{aligned}
$$


Now, we apply the FVM technique to the axisymmetric Navier-Stokes equations 5-8. The different terms are $\theta$-independent and thus $\int d \theta=2 \pi$ and we divide all the terms of the governing equations by $2 \pi$. We derive the system of algebraic equations from the model by Lyra [90] and Guardone [91]. We write $V o l_{i, j}=\iint R d r d z$ the volume of the control volume or cell of consideration, where $R$ is the distance of the cell centroid to the axis. Here, we wil consider a normalized grid, i.e. $\Delta r=0$ on edges 1 and 3 and $\Delta z=0$ on edges 2 and 4 . For each control volume $(i, j)$ of the grid we get the algebraic equation:

$$
\begin{aligned}
& \left(\frac{\partial U}{\partial t}\right)_{i, j} V o l_{i, j}+\int_{1} F_{r}(+) r_{i+\frac{1}{2}, j} d z-\int_{3} F_{r}(-) r_{i-\frac{1}{2}, j} d z+\int_{2} F_{z}(+) r d r-\int_{4} F_{z}(-) r d r \\
& =\int_{1} F_{r}^{v}(+) r_{i+\frac{1}{2}, j} d z-\int_{3} F_{r}^{v}(-) r_{i-\frac{1}{2}, j} d z+\int_{2} F_{z}^{v}(+) r d r-\int_{4} F_{z}^{v}(-) r d r+S_{i, j} V_{o l}
\end{aligned}
$$

\subsubsection{Time and Space Discretization: TVD MacCormack Scheme}

We now use the TVD MacCormack method presented in Fürst [92] that we adapt to the axisymmetric geometry. We solve the equations using a body-fitted structured mesh with quadrilateral cells. We denote $\vec{S}_{i+1 / 2, j}$ the normal vector to the interface between the cells $(i, j)$ and $(i+1, j)$ with the length of the vector equal to the area of the interface times the radius as defined above. It accounts for axisymmetric effects. In 2-D, the length of the vector is actually equal to the length of the interface. We suppose that the vector is oriented from cell $(i, j)$ to $(i+1, j)$ (Figure $5-5$ ). We operate similarly for $\vec{S}_{i-1 / 2, j}, \vec{S}_{i, j+1 / 2}$ and $\vec{S}_{i, j-1 / 2}$.

We write:

$$
\left\{\begin{array}{l}
\vec{S}_{i+1 / 2, j}=S_{i+1 / 2, j}^{z} \vec{e}_{z}+S_{i+1 / 2, j}^{r} \vec{e}_{r} \\
\vec{S}_{i, j+1 / 2}=S_{i, j+1 / 2}^{z} \vec{e}_{z}+S_{i, j+1 / 2}^{r} \vec{e}_{r} \\
\vec{S}_{i-1 / 2, j}=S_{i-1 / 2, j}^{z} \vec{e}_{z}+S_{i-1 / 2, j}^{r} \vec{e}_{r} \\
\vec{S}_{i, j-1 / 2}=S_{i, j-1 / 2}^{z} \vec{e}_{z}+S_{i, j-1 / 2}^{r} \vec{e}_{r}
\end{array}\right.
$$


Note that writing the numerical method using this particular formulation of the elementary volume and surface vectors enables the use of the exact same solver in two-dimensional geometry by simply dividing the latest quantities by $R_{i, j}$.

To make the derivation easier to read, we will now write that $F_{r}=F, F_{r}^{v}=F^{v}$, $F_{z}=G$ and $F_{z}^{v}=G^{v}$

The finite volume MacCormack scheme is then:

- Predictor

$$
\begin{aligned}
U_{i, j}^{n+\frac{1}{2}} & =U_{i, j}^{n}-\frac{\Delta t}{V o l_{i, j}}\left[S_{i+\frac{1}{2}, j}^{r} F_{i, j}^{n}+S_{i+\frac{1}{2}, j}^{z} G_{i, j}^{n}\right. \\
& +S_{i, j+\frac{1}{2}}^{r} F_{i, j}^{n}+S_{i, j+\frac{1}{2}}^{z} G_{i, j}^{n}-S_{i-\frac{1}{2}, j}^{r} F_{i-1, j}^{n} \\
& \left.-S_{i-\frac{1}{2}, j}^{z} G_{i-1, j}^{n}-S_{i, j-\frac{1}{2}}^{r} F_{i, j-1}^{n}-S_{i, j-\frac{1}{2}}^{z} G_{i, j-1}^{n}\right] \\
& +\Delta t\left[\operatorname{Visc}\left(U_{i, j}^{n}\right)+S\right]
\end{aligned}
$$

- Corrector

$$
\begin{aligned}
\overline{U_{i, j}^{n+1}} & =\frac{1}{2}\left[U_{i, j}^{n}+U_{i, j}^{n+1 / 2}-\frac{\Delta t}{V o l_{i, j}}\left[S_{i+\frac{1}{2}, j}^{r} F_{i+1, j}^{n+\frac{1}{2}}+S_{i+\frac{1}{2}, j}^{z} G_{i+1, j}^{n+\frac{1}{2}}\right.\right. \\
& +S_{i, j+\frac{1}{2}}^{r} F_{i, j+1}^{n+\frac{1}{2}}+S_{i, j+\frac{1}{2}}^{z} G_{i, j+1}^{n+\frac{1}{2}}-S_{i-\frac{1}{2}, j}^{r} F_{i, j}^{n+\frac{1}{2}} \\
& \left.\left.-S_{i-\frac{1}{2}, j}^{z} G_{i, j}^{n+\frac{1}{2}}-S_{i, j-\frac{1}{2}}^{r} F_{i, j}^{n+\frac{1}{2}}-S_{i, j-\frac{1}{2}}^{z} G_{i, j}^{n+\frac{1}{2}}\right]\right] \\
& +\frac{\Delta t}{2}\left[\operatorname{Visc}\left(U_{i, j}^{n+\frac{1}{2}}\right)+S\right]
\end{aligned}
$$

- Viscous Term

$$
\begin{aligned}
\operatorname{Visc}\left(U_{i, j}\right) & =\frac{1}{V o l_{i, j}}\left[S_{i+\frac{1}{2}, j}^{r} F_{i+\frac{1}{2}, j}^{v}+S_{i+\frac{1}{2}, j}^{z} G_{i+\frac{1}{2}, j}^{v}+S_{i, j+\frac{1}{2}}^{r} F_{i, j+\frac{1}{2}}^{v}+S_{i, j+\frac{1}{2}}^{z} G_{i, j+\frac{1}{2}}^{v}\right. \\
& \left.-S_{i-\frac{1}{2}, j}^{r} F_{i-\frac{1}{2}, j}^{v}-S_{i-\frac{1}{2}, j}^{z} G_{i-\frac{1}{2}, j}^{v}-S_{i, j-\frac{1}{2}}^{r} F_{i, j-\frac{1}{2}}^{v}-S_{i, j-\frac{1}{2}}^{z} G_{i, j-\frac{1}{2}}^{v}\right]
\end{aligned}
$$

For the evaluation of the viscous fluxes $F^{v}$ and $G^{v}$ at the interfaces we need to evaluate the velocity vector at the interface as well as the pressure, velocity and temperature derivatives. We use a central cell scheme to achieve both.

- TVD 
After the predictor and corrector, we add the TVD-type terms:

$$
\mathrm{U}_{\mathbf{i}, \mathbf{j}}^{\mathrm{n}+1}=\overline{\mathrm{U}_{\mathbf{i}, \mathbf{j}}^{\mathrm{n}+1}}+\mathrm{DW}_{\mathbf{i}, \mathbf{j}}^{1}+\mathrm{DW}_{\mathbf{i}, \mathbf{j}}^{2}
$$

computed as for a one dimensional TVD-type viscosity terms in the direction of change of $i$ and $j$. Several definitions are available to derive the $D W$ terms. The various model are presented further in this chapter.

\subsubsection{Time Stepping}

The TVD MacCormack scheme is implicit in time. However, to make sure that the scheme is always stable, we implement the so-called CFL condition:

$$
\Delta t=C F \operatorname{Lmin}\left(\frac{1}{\frac{\left|u_{z}(i, j)\right|+c_{i, j}}{\Delta z_{i, j}}+\frac{\left|u_{r}(i, j)\right|+c_{i, j}}{\Delta r_{i, j}}}\right)
$$

where $c_{i, j}$ is the local speed of sound and CFL is chosen between 0 and 1.

\subsubsection{Boundary Conditions}

The types of boundary conditions are the same for all the systems under study. The design imposes a condition on mass-flow-rate (or temperature+ velocity) at the inlet, a condition on pressure at the exit and a condition on temperature (or heat flux) on the wall. Also the gases of considerations are viscous, so we can assume a no-slip condition along the wall. Since the configuration chosen is axisymmetric, an condition should be expressed on the axis of rotation. In the approach to CFD solver we chose for our research project we use 'ghost' cells to impose the values at the geometrical boundaries.

- Inlet Boundary Condition At the inlet is given the velocity or mass-flow rate which depends on the reactors design. The inlet temperature is also given. An initial guess for the inlet pressure can be interpreted from typical operation pressure in systems. We create a ghost well downstream of the inlet plane where we impose the axial 
velocity equal to the inlet velocity and the radial velocity equal to zero

$$
u_{r}=0 \quad u_{z}=u_{\text {inlet }}
$$

In the case we would want to consider an already developed flow, the

$$
u_{z}(r)=u_{\text {inlet }}\left(1+1.44 \sqrt{f_{f}}+2.15 \sqrt{f_{f}} \log _{10}\left(1-\frac{r}{R}\right)\right)
$$

where $f_{f}$ is the friction factor defined by

$$
\left\{\begin{array}{lll}
f_{f}=\frac{16.0}{R e} & R e \leq 2000 & \text { (Blasius Law) } \\
f_{f}=0.0014+\frac{0.125}{R e^{0.32}} & R e>2000 & \text { (Koo's relation) }
\end{array}\right.
$$

- Exit Boundary Condition

At the exit we suppose given a static pressure

$$
p=p_{\text {exit }}
$$

and the normal derivative of the temperature

$$
\frac{\partial T}{\partial n}=\frac{\partial T}{\partial z}=0
$$

- Wall Boundary Condition

At an impermeable boundary, the flow of fluid relative to the boundary must be tangential to it. Since the flow is viscous, we consider the slip condition for which the tangential velocity equals the velocity of the wall.

$$
u_{r}=0 \quad u_{z}=u_{\text {wall }}
$$

For the systems we consider, the walls are immobile and therefore the tangential velocity equals zero.

To implement the numerical models we create fictive cells behind the wall. At the wall we also prescribe two types of thermal boundary conditions: 


\section{Temperature}

\section{Heat Flux}

Based on Fourier's law

$$
q^{\prime \prime}=-k_{w}\left(\frac{\partial T}{\partial r}\right)_{g a s}
$$

where $k_{w}$ is the conductivity of the gas estimated at the wall.

Then the gradient of Equation 5-38 is rewritten

$$
q^{\prime \prime}=k_{w} \frac{T_{2}-T_{1}}{r_{2}-r_{1}}
$$

where subscripts 1 and 2 refer to the outside and inside wall boundary mesh points, respectively. Because non-constrained integration of the first derivative of temperature provides an infinite number of solutions it is very difficult to implement the boundary condition. To overcome the shortcut, we use the enthalpy-rebalancing technique developed by Anghaie and Chen [93]. It is based on the observation that under steady-state the gas enthalpy increase along the wall surface is equal to the heat removed from the wall:

$$
\left(T_{b}\right)_{i+1}=\left(T_{b}\right)_{i}+\frac{P(\Delta z)_{i} q_{i}^{\prime \prime}}{S\left(c_{P}\right)_{i}(\rho u)_{i}}
$$

where $T_{b}$ is the bulk temperature, $P=2 \pi R$ is the perimeter of the channel and $S=\pi R^{2}$ the flow cross-sectional area. The previous bulk $\left(\left(T_{b}\right)_{i+1}\right)_{0}$ is calculated from temperature fields for each iteration. We then define a convergence parameter $\delta$ :

$$
\delta=\frac{\left(T_{b}\right)_{i+1}}{\left(\left(T_{b}\right)_{i+1}\right)_{0}}
$$

During the transitional state, the product $\rho u$ is smaller that in the steady-state. Therefore, $\delta>1$. When the mass flux approximates its steady state value, $\delta$ starts approaching its asymptotical value of 1 . At this point can we calculate the wall boundary temperature from Equation 5-39. Then, as soon as he condition $\delta=1$ is verified, the solution is converged. 
- Axis Boundary Condition

we suppose that there is no fluid flow and scalar flux across the boundary: the normal velocity is set to be zero at the boundary. Practically, we implement this boundary condition by using a fictive axis cell, as for the wall. The fluxes in this cell are equal to the fluxes in the adjacent cell.

$$
F_{\text {fictive }}=F_{\text {adj }} \text { and, } G_{\text {fictive }}=G_{\text {adj }}
$$

where $F_{a d j}$ and $G_{a d j}$ are the fluxes in the cell adjacent to the boundary.

\subsubsection{Limiters and Total Variation Diminishing Condition}

The concept of limiters has been introduced to prevent the generation of potential oscillations rather than damping them. If we consider the simple conservative form of the

Euler equation $\frac{\partial U}{\partial t}+\frac{\partial F}{\partial x}=0$ the total variation quantity of a variable such as $U$ is defined by:

$$
T V(U)=\int\left|\frac{\partial U}{\partial x}\right| d x
$$

where in a discrete form, the total variation of numerical solution may be expressed by:

$$
T V\left(U^{n}\right)=\sum_{-\infty}^{+\infty}\left|u_{i+1}^{n}-u_{i}^{n}\right|
$$

A numerical scheme is said to be Total Variation Diminishing (TVD) in time if

$$
T V\left(u^{n+1}\right) \leq T V\left(U^{n}\right)
$$

If a scheme is monotone at time 'n' it has to remain monotone at time 'n+1'. When numerical oscillations are generated the global rate of variation of the function over the domain increases, and so is the total variation quantity. TVD states that the total variation quantity cannot grow in time. For a MUSCL second-order scheme to satisfy the TVD condition the fluxes terms in the differential equations are multiplied by a non-linear function. The function is to be chosen such that the PDE satisfies the TVD condition. 
The flux limiter function takes values between 0 and 1 . When in a smooth region, the gradients are small and the flux limiter should be closed to 1. Near a discontinuity, the gradients can take values close to infinity and the value of the limiter should be close to zero. For a discretization at point i given by:

$$
\phi_{i}^{n+1}=\phi_{i}^{n}-\nu \delta \phi_{i-1}^{n}-\frac{\nu}{2}(1-\nu)\left(\delta \phi_{i-1}^{n}-\delta \phi_{i-2}^{n}\right)
$$

the flux limiter $\Psi(r)$ is introduced such that

$$
\phi_{i}^{n+1}=\phi_{i}^{n}-\nu \delta \phi_{i-1}^{n}-\frac{\nu}{2}(1-\nu)\left(\Psi\left(r_{i-1}^{+}\right) \delta \phi_{i-1}^{n}-\Psi\left(r_{i-2}^{++}\right) \delta \phi_{i-2}^{n}\right)
$$

with $r_{i-1}^{+}=\frac{\delta \phi_{i+1}}{\delta \phi_{i-1}}$ and $r_{i-2}^{++}=\frac{\delta \phi_{i-1}}{\delta \phi_{i-2}}$.

In the stenciling formulas, the limited gradients are in all cases a function of the appropriate gradient ratios. The gradient limiters can be selected from the list given below (5-43-5-46). Note that all the limiters return 0 if the gradient ratio is negative. The case where the gradient switches direction indicates that there is a shock within the cell range and the local reconstruction is reduced for first order in this instance. The most common limiters are [38]:

- Min-mod limiter

$$
\Psi_{m}(r)= \begin{cases}\min (r, 1) & \text { if } r>0 \\ 0 & \text { if } r \leq 0\end{cases}
$$

- Superbee limiter

$$
\Psi_{s}(r)=\max [0, \min (2 r, 1), \min (r, 2)]
$$

- Van Albada Limiter

$$
\Psi_{v a}(r)=\frac{r+r^{2}}{1+r^{2}}
$$

- Van Leer Limiter

$$
\Psi_{v l}(r)=\frac{2 r}{1+r}
$$


In the MacCormack method presented by Fürst there are three types of methods to derive the TVD-type terms $D W$. We are interested in two of them: the full MacCormack scheme gives very accurate results but is computationally expensive. A modified Causon scheme [94] is also proposed which accelerates consequently convergence of the solution at the expense of accuracy.

\subsubsection{Full MacCormack scheme}

The full MacCormack scheme is close to a Roe scheme [46]. We derive the TVD-term in direction 1 , in the case of a two-dimensional problem. The giverning equation in its compact form was derived Equation 5-8. We build a matrix $A$ such that

$$
A_{i+\frac{1}{2}, j}=S_{i+\frac{1}{2}, j}^{r}\left(\frac{\partial F}{\partial U}\right)_{i+\frac{1}{2}, j}+S_{i+\frac{1}{2}, j}^{z}\left(\frac{\partial G}{\partial U}\right)_{i+\frac{1}{2}, j}
$$

$A$ has $m$ real eigenvalues and $m$ linearly independent right vectors. The eigenvalues of $A_{i+\frac{1}{2}, j}$ are:

$$
\left\{\begin{array}{l}
a_{i+\frac{1}{2}, j}^{(1)}=S_{i+\frac{1}{2}, j}^{r}\left(u_{r}\right)_{i+\frac{1}{2}, j}+S_{i+\frac{1}{2}, j}^{z}\left(u_{z}\right)_{i+\frac{1}{2}, j}-a_{i+\frac{1}{2}, j}\left|\vec{S}_{i+\frac{1}{2}, j}\right| \\
a_{i+\frac{1}{2}, j}^{(2)}=S_{i+\frac{1}{2}, j}^{r}\left(u_{r}\right)_{i+\frac{1}{2}, j}+S_{i+\frac{1}{2}, j}^{z}\left(u_{z}\right)_{i+\frac{1}{2}, j} \\
a_{i+\frac{1}{2}, j}^{(3)}=S_{i+\frac{1}{2}, j}^{r}\left(u_{r}\right)_{i+\frac{1}{2}, j}+S_{i+\frac{1}{2}, j}^{z}\left(u_{z}\right)_{i+\frac{1}{2}, j} \\
a_{i+\frac{1}{2}, j}^{(4)}=S_{i+\frac{1}{2}, j}^{r}\left(u_{r}\right)_{i+\frac{1}{2}, j}+S_{i+\frac{1}{2}, j}^{z}\left(u_{z}\right)_{i+\frac{1}{2}, j}+a_{i+\frac{1}{2}, j}\left|\vec{S}_{i+\frac{1}{2}, j}\right|
\end{array}\right.
$$

We can decompose the matrix A into

$$
A=R \Lambda R^{-1}
$$

where, $\Lambda$ is the diagonal matrix composed of the eigenvalues of $A . r_{i, j}^{ \pm}$are vectors with $m$ components and the $l$ th components are written

$$
\left(r_{i, j}^{+}\right)^{(l)}=\frac{\left(R_{i-\frac{1}{2}, j}^{-1}\left(U_{i, j}-U_{i-1, j}\right)\right)^{(l)}}{\left(R_{i+\frac{1}{2}, j}^{-1}\left(U_{i+1, j}-U_{i, j}\right)\right)^{(l)}}
$$




$$
\left(r_{i, j}^{-}\right)^{(l)}=\frac{\left(R_{i+\frac{1}{2}, j}^{-1}\left(U_{i+1, j}-U_{i, j}\right)\right)^{(l)}}{\left(R_{i-\frac{1}{2}, j}^{-1}\left(U_{i, j}-U_{i-1, j}\right)\right)^{(l)}}
$$

The vectors of conservative variables $U$ are taken at the old time step $n$. To evaluate the values of $A$ and $R$ at the interfaces, we can use Roe's averaging technique

$$
U_{i+\frac{1}{2}}=\frac{\sqrt{\rho_{i}} U_{i}+\sqrt{\rho_{i+1}} U_{i+1}}{\sqrt{\rho_{i}}+\sqrt{\rho_{i+1}}}
$$

The viscosity coefficients $\tilde{G}$ are the $m \mathrm{x} m$ diagonal matrices which elements are given by

$$
\tilde{G}^{ \pm}\left(r_{i, j}^{ \pm}\right)^{(l, l)}=\frac{1}{2} \frac{\left|a^{(l)}\right| \Delta t}{\Delta x}\left(1-\frac{\left|a^{(l)}\right| \Delta t}{\Delta x}\right)\left[1-\Psi\left(\left(r_{i, j}^{ \pm}\right)^{(l)}\right)\right]
$$

and $a^{(l)}$ is the $l$ th eigenvalue of matrix $A$ given by system $5-47 . \Psi$ is the limiter function. We typically choose the min-mod limiter defined in Equation 5-43. Finally,

$$
\begin{aligned}
D W_{i, j}^{1} & =R_{i+\frac{1}{2}, j}\left[\tilde{G}^{+}\left(r_{i, j}^{+}\right)+\tilde{G}^{-}\left(r_{i+1, j}^{+}\right)\right] R_{i+\frac{1}{2}, j}^{-1}\left(U_{i+1, j}-U_{i, j}\right) \\
& -R_{i-\frac{1}{2}, j}\left[\tilde{G}^{+}\left(r_{i-1, j}^{+}+\tilde{G}^{-}\left(r_{i, j}^{+}\right)\right] R_{i-\frac{1}{2}, j}^{-1}\left(U_{i, j}-U_{i-1, j}\right)\right.
\end{aligned}
$$

\subsubsection{Modified Causon limiter}

The TVD-type limiter in the direction 1 is given by:

$$
\begin{gathered}
D W_{i, j}^{1}=\left[\bar{G}^{1+}\left(r_{i, j}^{1+}\right)+\bar{G}^{1-}\left(r_{i+1, j}^{1-}\right)\right]\left(U_{i+1, j}^{n}-U_{i, j}^{n}\right) \\
-\left[\bar{G}^{1+}\left(r_{i-1, j}^{1+}\right)+\bar{G}^{1-}\left(r_{i, j}^{1-}\right)\right]\left(U_{i, j}^{n}-U_{i-1, j}^{n}\right) \\
r_{i, j}^{1+}=\frac{\left\langle U_{i, j}^{n}-U_{i-1, j}^{n}, U_{i+1, j}^{n}-U_{i, j}^{n}\right\rangle}{\left\langle U_{i+1, j}^{n}-U_{i, j}^{n}, U_{i+1, j}^{n}-U_{i, j}^{n}\right\rangle} \\
r_{i, j}^{1-}=\frac{\left\langle U_{i, j}^{n}-U_{i-1, j}^{n}, U_{i+1, j}^{n}-U_{i, j}^{n}\right\rangle}{\left\langle U_{i, j}^{n}-U_{i-1, j}^{n}, U_{i, j}^{n}-U_{i-1, j}^{n}\right\rangle} \\
\bar{G}^{1 \pm}\left(r_{i, j}^{1 \pm}\right)=\frac{1}{2} C\left(\nu_{i, j}\right)\left[1-\Phi\left(r_{i, j}^{1 \pm}\right)\right]
\end{gathered}
$$


where $\Phi$ is the flux limiter function. From the 1-D validation case, we chose the Van Albada technique (see equation).

$$
\begin{gathered}
C\left(\nu_{i, j}\right)= \begin{cases}\nu_{i, j}\left(1-\nu_{i, j}\right) & \text { for } \nu_{i, j} \leq 0.5 \\
0.25 & \text { for } \nu_{i, j}>0.5\end{cases} \\
\nu_{i, j}=\frac{\Delta t}{\Delta z} \Psi\left(\min \left(\left|u_{i, j}^{1}-c_{i, j}\right|,\left|u_{i, j}^{1}\right|,\left|u_{i, j}^{1}+c_{i, j}\right|\right)\right.
\end{gathered}
$$

where $u^{1}$ is the velocity in the direction of the change of the index $1, c$ is the local speed of sound, $\Delta z$ is the approximation of the cell length in the direction of the change and $\Psi$ is the entropy correction function:

$$
\Psi(z)=\left\{\begin{array}{lll}
|z| & \text { for } \quad|z|>\epsilon_{e} \\
\frac{z^{2}+\epsilon_{e}^{2}}{2 \epsilon_{e}} & \text { for }|z| \leq \epsilon_{e}
\end{array}\right.
$$

with $\epsilon_{e}=10^{-3}$.

\subsection{Remark on the Practical Implementation of the Real Gas Properties - Algorithm}

The real challenge when implementing the real gas properties is to get convergence of the solver, while the diverse properties such as heat capacity, thermal conductivity and viscosity are very sensitive to small temperature and pressure variations.

Figure 5-6 presents a schematics of the algorithm that we used to implement the real property values. At time $n$, we have the values of $U$ which were derived at the previous time step, as well as the values of $p$ and $T$ and all the properties (k, $c_{p}$ and $\mu$ ).

We then derive the diffusion fluxes $F$ and $G$ and the viscous fluxes $F^{v}$ and $G^{v}$ at time $n$ which is the initial time for the iteration step.

Using the TVD-MacCormack scheme we get the value of the vector of conservative variables at the intermediate time step, classically noted $n+\frac{1}{2}: U^{n+\frac{1}{2}}$.

We then derive the value of the predicted pressure using the ideal-gas relation 5-10. However, we note that in the relation $\mathrm{p}$ is a function of total energy, velocity vector and 
specific heat ratio. Both energy and velocity at the prediction step are derived with exactitude. However, the specific heat is a function of pressure and temperature. We do not know the exact value of it at $n+\frac{1}{2}$ and therefore, the value of pressure obtained is also an estimation. The same way we derive the temperature at the predictor step using the ideal gas law 5-9.

From these predicted values of pressure and temperature, we run the property subroutine where the correlations of all properties are expressed as a function of temperature and pressure and are continuous over the range of operations.

With the new value of the specific heat ratio $(\gamma)$ we obtain, we recalculate an estimation of the pressure and temperature.

If the update value of both pressure and temperature is the same as the previous prediction, then we can consider that the loop on the properties is over. Otherwise, the properties are re-calculated and the loop on properties iterated until the values of pressure and temperature are converged in all the grid calculation nodes.

Once the loop on properties is converged, the fluxes are updated with the all the predicted values in the predictor step.

We now are ready to perform the corrector step, and derive the values of $U$ at time $n+1$, using the values of the vector of conservative variables and fluxes at time $n+\frac{1}{2}$.

Then, the flux limiter is determined. We calculate one flux limiter per modeling dimension, therefore, for our two-dimensional axisymmetric model, we will have to derive two flux limiters one in the axial and one in the radial dimension.

From the values of the corrector and the flux limiters, we derive the value of the vector of conservative variables at time $n+1$ with equation 5-32.

To get the values of pressure and temperature as well as fluid properties at time $n+1$ we need to iterate again on the properties, in the same fashion as for the predictor step.

Once the convergence of the property loop is obtained, the complete solution and flow map at time $n+1$ is calculated. 
We iterate on the time-loop until steady-state is reached: Once steady-state is reached, the flow maps remains the same even when more iterations are implemented.

\subsection{Validation Cases: The Riemann Shock Tube}

\subsubsection{Presentation of the Test Case}

The shock tube or Riemann problem constitutes a particularly interesting test case since it presents an exact solution to the one-dimensional Euler equation and involves simultaneously a shock wave, a contact discontinuity and an expansion fan. The Riemann

problem can be realized experimentally by the sudden breakdown of a diaphragm in a long one-dimensional tube. Before time of breakdown $(t=0)$ the diaphragm separates two gas states at two different pressures and densities. We consider air at room temperature in the tube. The governing equations for this test case are the one-dimensional time-dependent Euler equations with an ideal Equation of State. Their conservative form is:

$$
\frac{\partial \mathbf{U}}{\partial t}+\frac{\partial \mathbf{F}(\mathbf{U})}{\partial x}=0
$$

where $\mathbf{U}$ and $\mathbf{F}(\mathbf{U})$ are the vectors of conserved variables and fluxes, given respectively by:

$$
\mathbf{U}=\left[\begin{array}{c}
\rho \\
\rho u \\
E
\end{array}\right], \mathbf{F}=\left[\begin{array}{c}
\rho u \\
\rho u^{2}+p \\
u(E+p)
\end{array}\right]
$$

The ideal-gas law applies and the specific heat ratio of air remain constant: $\gamma=1.4$. If the diaphragm is located a position $x_{0}$ then the initial condition are the following:

$$
\left\{\begin{array}{cccc}
u=u_{L} & p=p_{L} & \rho=\rho_{L} & x<x_{0} \\
u=u_{R} & p=p_{R} & \rho=\rho_{R} & x>x_{0}
\end{array}\right.
$$

Schematic of the experiment is presented Figure 5-7. The analytical solution is interfered form the theory of the compressible fluid flows. Hirsch [38] gives the theoretical profiles for different initial pressure ratios and at different times. We also derive a FORTRAN code which computes the theoretical answer. It can be found Appendix A. 


\subsubsection{Numerical Method}

A one-dimensional code using the theory presented in the previous section is written and results are compared to the analytical solution. We use the TVD MacCormack implicit scheme to derive the flow behavior. For the one-dimensional solver the predictor and corrector steps are now written

$$
\left\{\begin{array}{l}
U_{i}^{n+\frac{1}{2}}=U_{i}^{n}-\frac{\Delta t}{\Delta x}\left(F_{i}^{n}-F_{i+1}^{n}\right) \\
U_{i}^{n+1}=\frac{1}{2}\left[U_{i}^{n}+U_{i}^{n+\frac{1}{2}}-\frac{\Delta t}{\Delta x}\left(F_{i+1}^{n+\frac{1}{2}}-F_{i}^{n+\frac{1}{2}}\right)\right]
\end{array}\right.
$$

In the case of the full TVD-MacCormack we need to derive the matrix $A=\frac{\partial F}{\partial U}$, its eigenvalues and right eigenvectors:

- Matrix $A$

$$
A_{i}=\left[\begin{array}{ccc}
0 & 1 & 0 \\
\frac{1}{2} u_{i}^{2}(\gamma-3) & (3-\gamma) u_{i} & \gamma-1 \\
u_{i}^{3}(\gamma-1)-\gamma E_{i} u_{i} & \gamma E_{i}-\frac{3}{2}(\gamma-1) u_{i}^{2} & \gamma u_{i}
\end{array}\right]
$$

- Eigenvalues

$$
\left\{\begin{array}{l}
a_{i}^{(1)}=u_{i} \\
a_{i}^{(2)}=u_{i}+c_{i} \\
a_{i}^{(3)}=u_{i}-c_{i}
\end{array}\right.
$$

- Right Eigenvectors

$$
R_{i}=\left[\begin{array}{ccc}
1 & 1 & 1 \\
u_{i} & u_{i}+c_{i} & u_{i}-c_{i} \\
\frac{u_{i}^{2}}{2} & \frac{u_{i}^{2}}{2}+c_{i} u_{i}+\frac{c_{i}^{2}}{\gamma-1} & \frac{u_{i}^{2}}{2}-c_{i} u_{i}+\frac{c_{i}^{2}}{\gamma-1}
\end{array}\right]
$$




\subsubsection{Results}

The CFD solver results are compared to analytical results of the following Riemann case:

$$
\left\{\begin{array}{lccc}
\text { left side : } & \rho=1.0 & u=0 & p=1.0 \\
\text { right side }: & \rho=0.125 & u=0 & p=0.1
\end{array}\right.
$$

The analytical and numerical profiles of density, velocity and pressure are compared. They are given Figure 5-8. We see that the gas behavior trendline is perfectly followed when using with our TVD MacCormack solver with modified Causon scheme. However, the limiter lacks a little bit of accuracy since a few numerical oscillations are generated in the viscidity of the property discontinuities. It would be a problem in applications where shocks are generated. However, in the system we study, no shocks should be generated. We need a solver which can simulate accurately flow as they become close to $M=1$ but we should never have formation and propagation of discontinuities in the gas.

This case on the very traditional Riemann Shock tube benchmark validates the numerical technique we chose for applications to advanced nuclear systems such as the two systems presented Chapter 6 and 7 .

\subsubsection{On the influence of the gas}

We run the Riemann solver for diverse gases of interest at $300 \mathrm{~K}$. Their heat ratios are the following

$$
\left\{\begin{array}{l}
\gamma_{a i r}=\gamma_{H_{2}}=1.4 \\
\gamma_{H e}=1.67 \\
\gamma_{C O_{2}}=1.19 \\
\gamma_{U F_{4}}=1.08
\end{array}\right.
$$

The profiles of density, velocity and pressure at $\mathrm{t}=6.1 \mathrm{~ms}$ with the initial condition $5-62$ are shown figure 5-9. The graph shows the importance of the value of the heat ratio on the evolution of the properties in the shock tube. Shock fronts and expansion waves propagate faster for gases with higher heat ratios. They lead to smaller amplitude of the shock fields, 
as seen on the velocity graph: For Helium, which has the higher heat ratio, the resulting velocity burst is smaller than for uranium tetrafluoride, which has the smallest value of gamma. Also, for higher values of gamma, there are almost no non-physical oscillations generated in areas of discontinuity. For $\gamma=1.08$ the solver is not able to capture the shocks properly. Therefore the limiter does not work as well. However, since the system we study should not exhibit propagating shocks and discontinuities in normal operation, we will consider that the results obtained with the solvers show the adequacy of out solver for the systems we will consider in our study. 


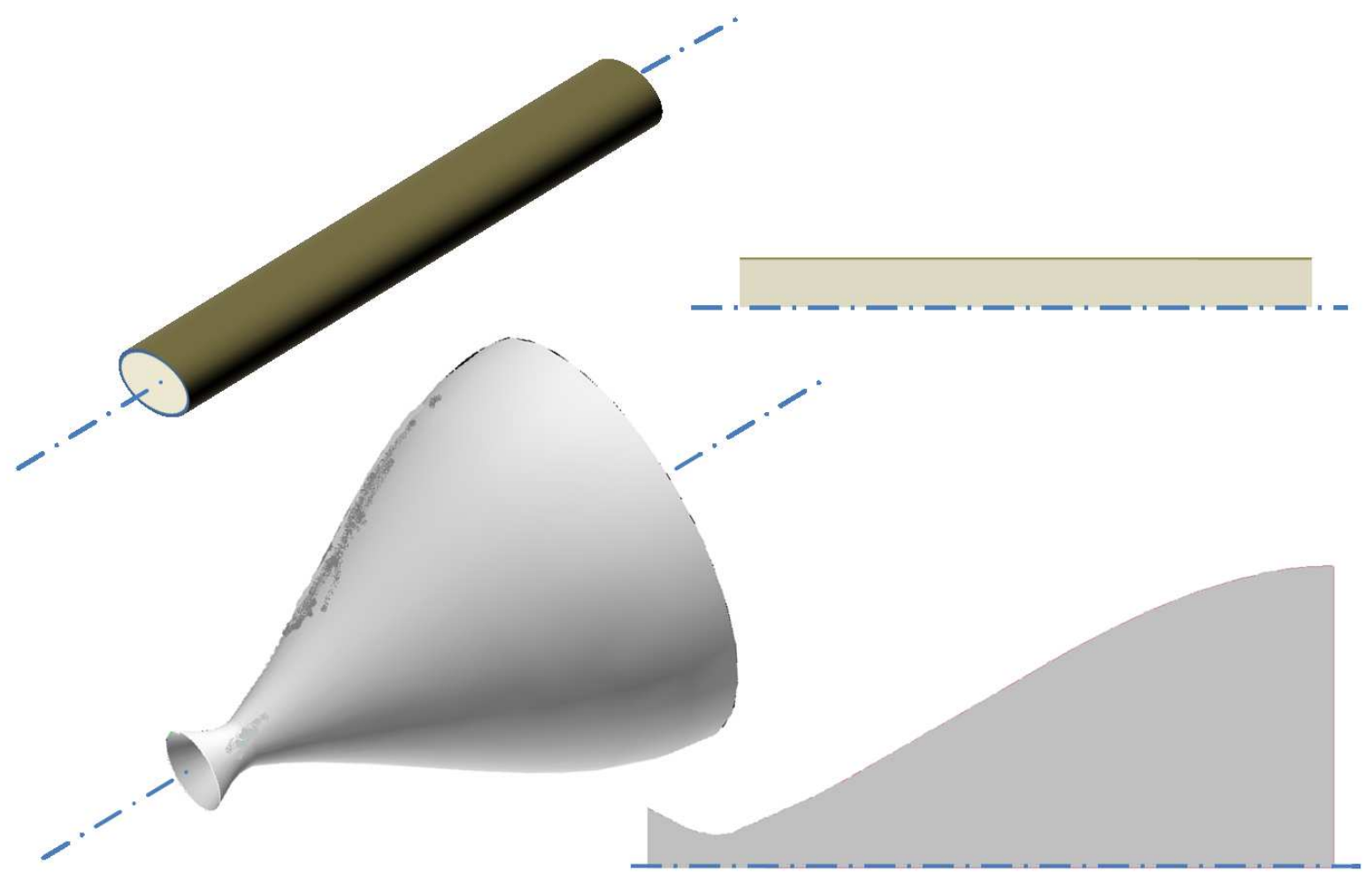

Figure 5-1: Sketch of the axisymmetric representation of the 3-D channel 


\begin{tabular}{|c|c|c|c|c|}
\hline$\stackrel{(5,1)}{\bullet}$ & $\stackrel{(5,2)}{\bullet}$ & $\stackrel{(5,3)}{\bullet}$ & $\stackrel{(5,4)}{\bullet}$ & $\stackrel{(5,5)}{\bullet}$ \\
\hline$(\stackrel{4,1)}{\bullet}$ & $\stackrel{(4,2)}{-}$ & $\stackrel{(4,3)}{\bullet}$ & $(4,4)$ & $\stackrel{(4,5)}{\bullet}$ \\
\hline$\stackrel{(3,1)}{\bullet}$ & $\stackrel{(3,2)}{\bullet}$ & $\stackrel{(3,3)}{-}$ & $\stackrel{(3,4)}{\bullet}$ & $\stackrel{(3,5)}{\bullet}$ \\
\hline$\stackrel{(2,1)}{\bullet}$ & $\stackrel{(2,2)}{\bullet}$ & $\stackrel{(2,3)}{\bullet}$ & $\stackrel{(2,4)}{\bullet}$ & $\stackrel{(2,5)}{\bullet}$ \\
\hline$\stackrel{(1,1)}{\bullet}$ & $\stackrel{(1,2)}{\bullet}$ & $\stackrel{(1,3)}{\bullet}$ & $\stackrel{(1,4)}{\bullet}$ & $\stackrel{(1,5)}{\bullet}$ \\
\hline
\end{tabular}

Figure 5-2: Location of the numerical nodes for a 5x5 domain 


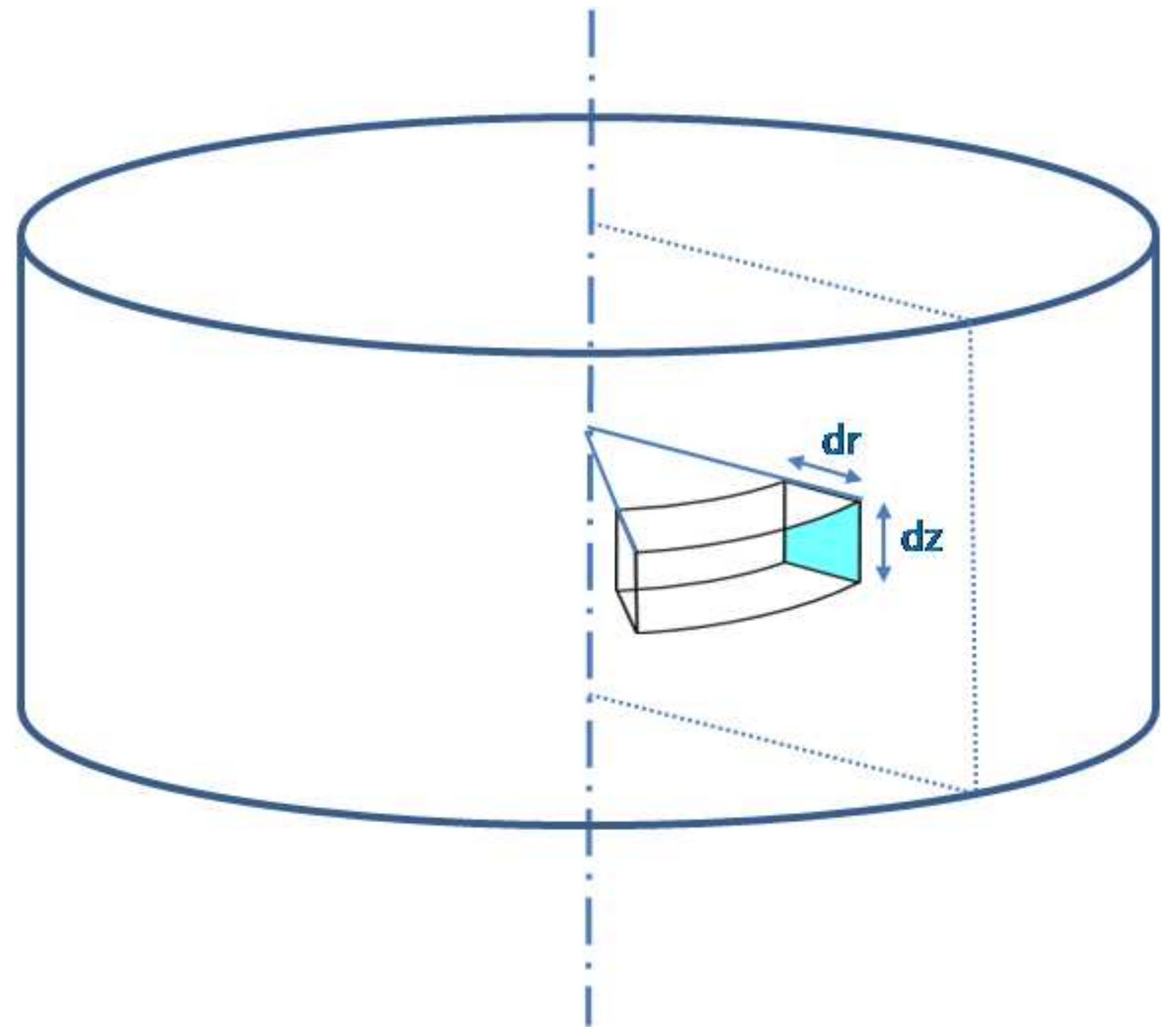

Figure 5-3: Elementary volume (blue area) for integration in the axisymmetric configuration 


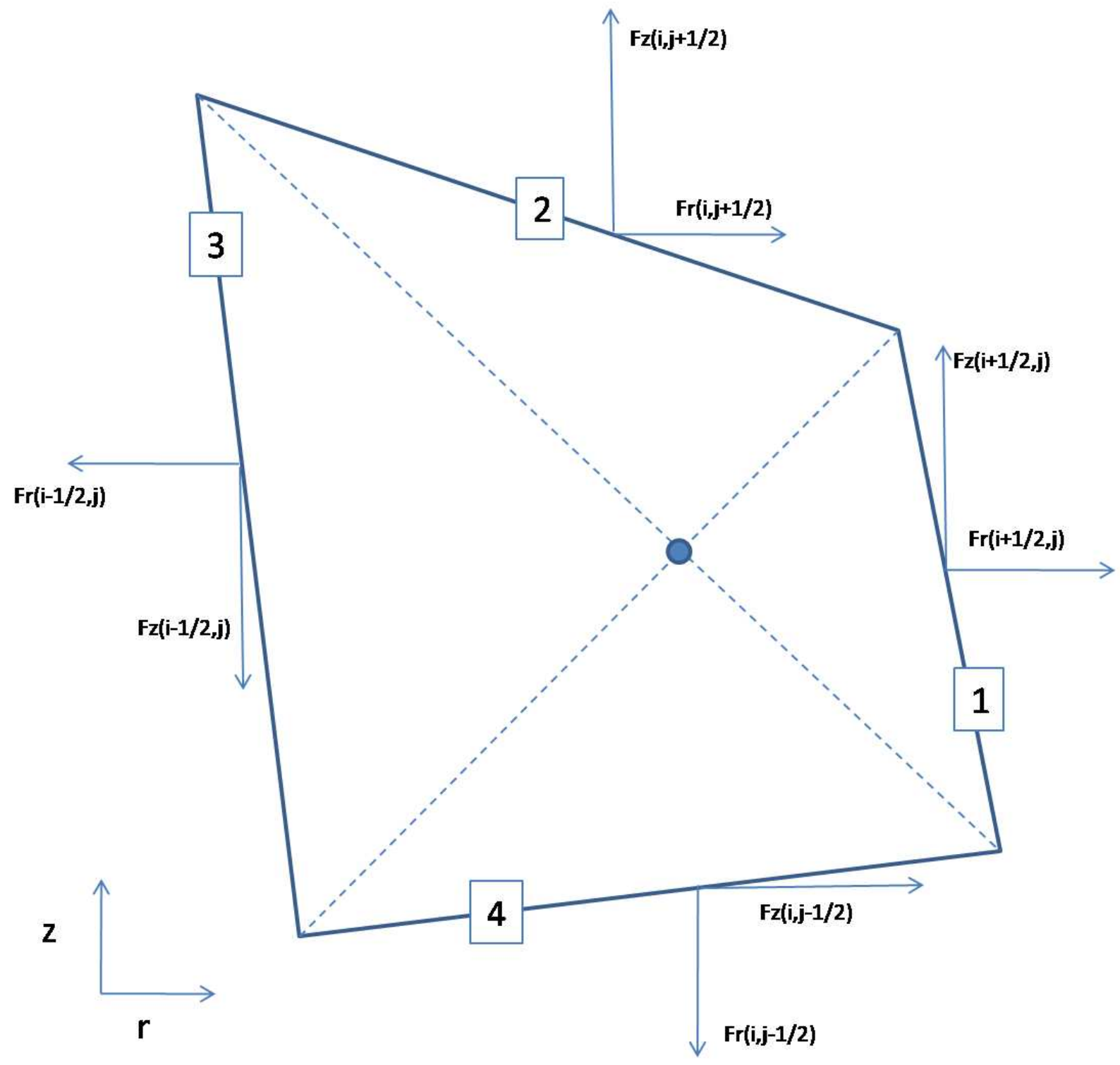

Figure 5-4: Details of the notations of the axisymmetric cell $(i, j)$ 


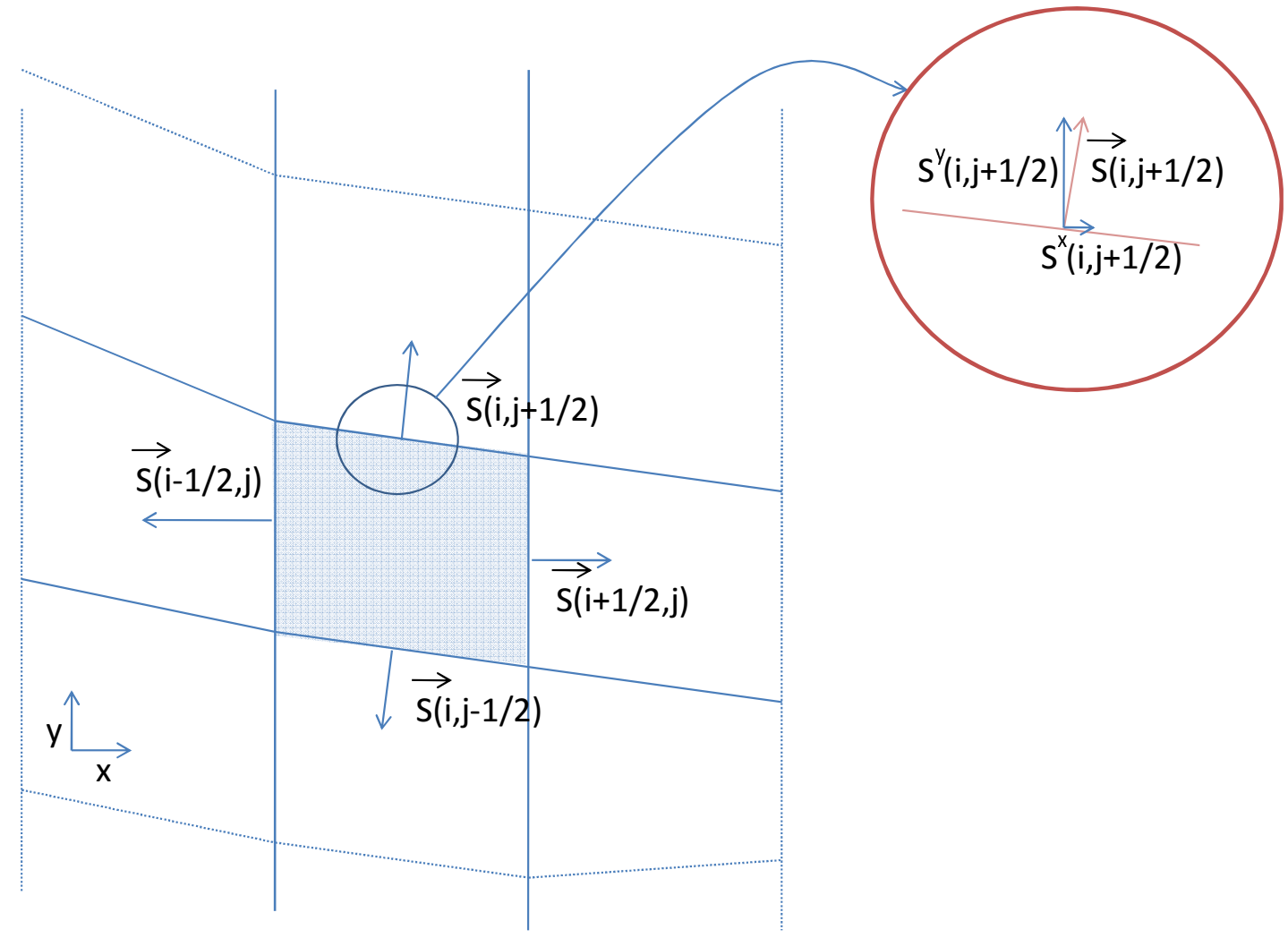

Figure 5-5: Nomenclature for the surface vectors 


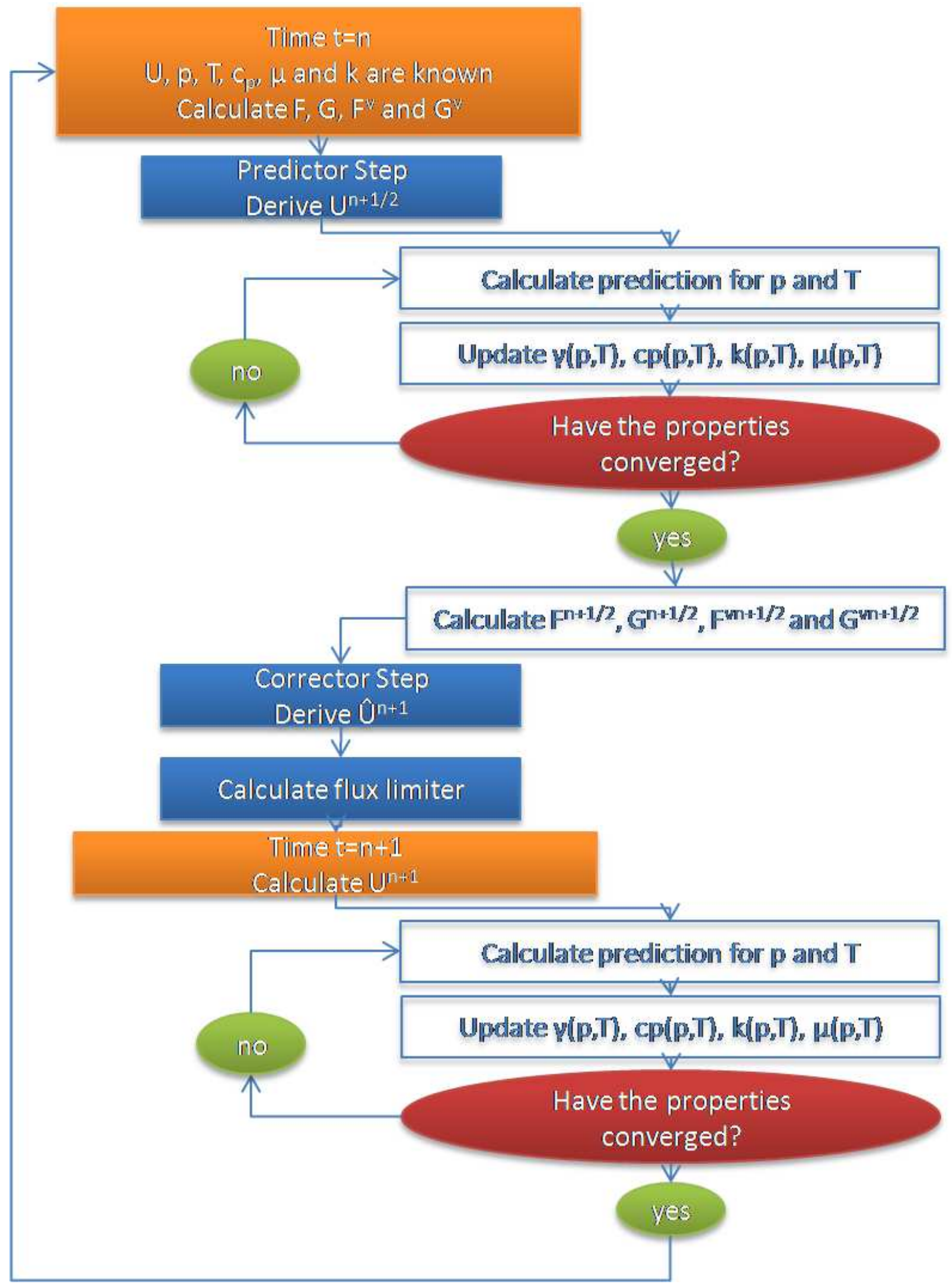

Figure 5-6: Algorithm which shows how the real properties are implemented and computed 


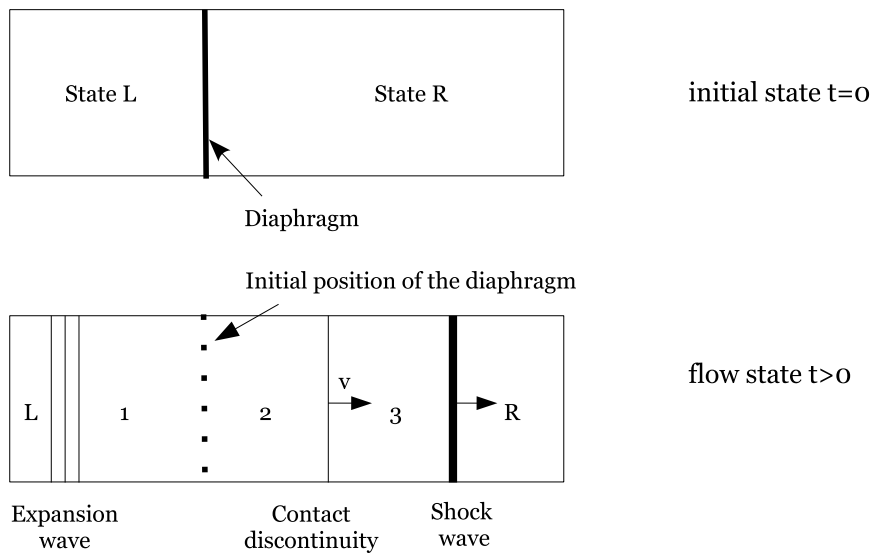

Figure 5-7: The shock tube experiment: Above, at time $\mathrm{t}=0$ a diaphragm separates the gases in two different states. Below, when the diaphragm is removed, blabla
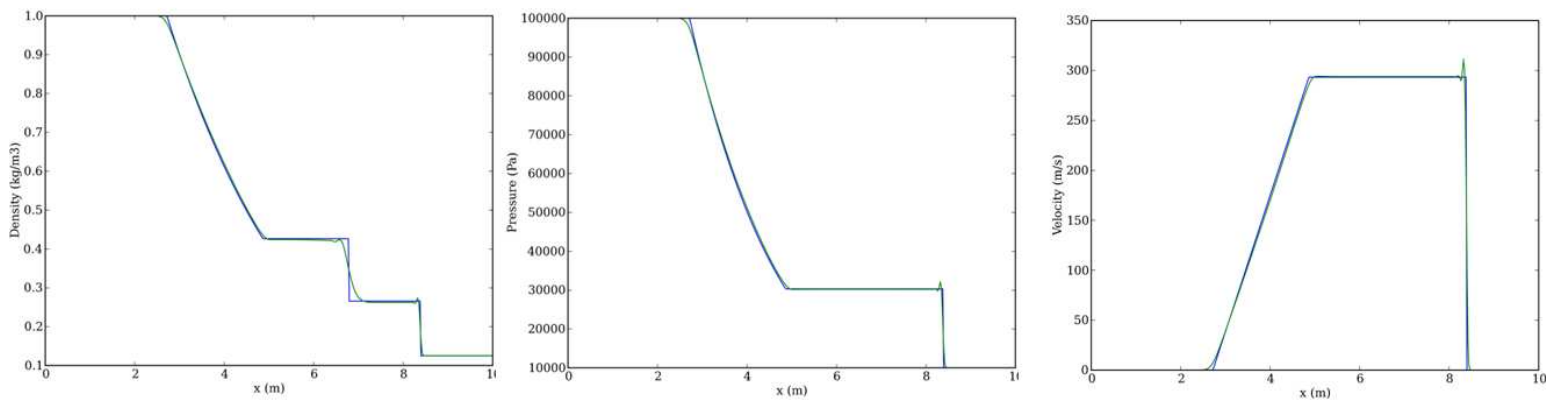

Figure 5-8: Shock tube profile at $\mathrm{t}=6.1 \mathrm{~ms}$ in case $5-62$. The blue curves are the theoretical profiles and the green curves the profiles computed with our Euler solver 


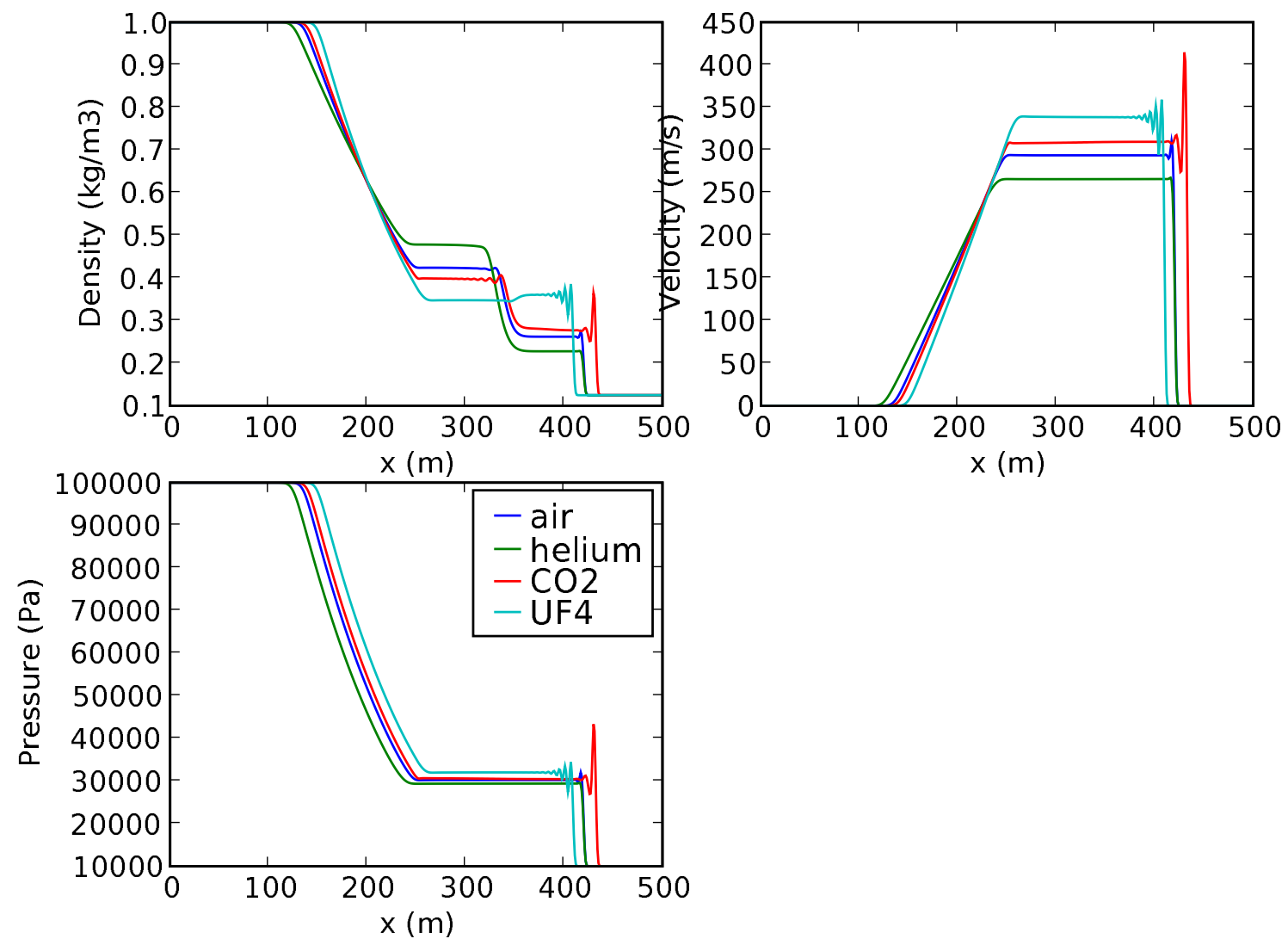

Figure 5-9: Properties profile at $\mathrm{t}=6.1 \mathrm{~ms}$ in case 5-62 for air, helium, carbon dioxide and uranium tetrafluoride. 


\section{CHAPTER 6 \\ CFD STUDY OF THE COOLANT CHANNELS OF A PRISMATIC VHTR}

In the scope of the Generation IV of reactors, the United States have decided to develop the Very High Temperature Gas-Cooled Reactor. On a thermal-hydraulics standpoint, some of the main characteristics are:

- Coolant: helium under single phase (gas)

- Moderator: Graphite block matrix

Helium heat transfer and transport coefficients (such as specific heat, thermal conductivity) are one of the highest of all gases. Helium is also chemically neutral and does not exhibit phase changes. It is nearly transparent to neutrons, which limits the reactivity effects associated with variations in coolant density. Therefore, helium is the preferred candidate for VHTR coolant and working fluid.

The operating pressure of the core is determined by considerations of core energy extraction (low density of helium) and thermodynamic efficiency. Preliminary studies determined the optimal value to be approximately $7 \mathrm{MPa}$.

The core of prismatic VHTRs is composed of a fuel and moderator matrix mostly composed of graphite. Helium flows through cylindrical channels pierced inside the matrix. Typically, the gas enters the core at $400^{\circ} \mathrm{C}$ and is heated to temperatures as high as $1,000^{\circ} \mathrm{C}$.

Characteristics of the flow of helium in the cooling channel is critical: the pressure drop over the core influences the rest of the system, the heat extracted from the fuel should be high enough to limit the maximum fuel temperature to $1,600^{\circ} \mathrm{C}$.

Figure 6-1 shows a schematic representation of the system. The geometrical parameters were determined at from Argonne National Laboratory [95]. The diameter and active length of the coolant channel are respectively:

$$
\left\{\begin{array}{l}
D=0.0159 \mathrm{~m} \\
L=7.93 \mathrm{~m}
\end{array}\right.
$$


The mass flow rate in a single coolant channel has not been determined yet. However, we can estimate that the Mach number of the flow should not be greater than 0.1. If we used an aerospace modeling approach, we could typically consider the flow as incompressible since the Mach number is smaller than 0.3. However, in the case of the flow in a coolant channel, this rule-of-thumb does not apply: if we consider that the walls of the channel are made of aluminum, therefore the roughness of the walls is approximately equal to $0.0013 \mathrm{~mm}$. The pressure drop resulting in a simple Bernoulli analysis is

$$
\Delta P=4.43 \text { bar }
$$

Therefore, the pressure variation across the length of the core is large and will greatly influence the density of the gas. Even if the Mach number is small, we have to consider a compressible flow because of the large variation in pressure and temperature across the system.

For a set of inlet temperature and pressure of $400^{\circ} \mathrm{C}$ and $7 \mathrm{MPa}$ respectively, the helium properties are:

$$
\left\{\begin{array}{l}
\mu=3.86 * 10^{-5} \text { Pa.s } \\
\rho=4.31 \mathrm{~kg} / \mathrm{m}^{3} \\
a=1544.4 \mathrm{~m} / \mathrm{s}
\end{array}\right.
$$

which leads to a Reynolds number of:

$$
R e=3.46 * 10^{5}
$$

Even in the most conservative version of the channel $(\mathrm{M}=0.1)$ the flow is undoubtedly turbulent. Thus, we model the turbulent effects in the coolant channel with the k- $\epsilon$ model (Section 5.1.5.2). 
We perform the analysis of the prismatic VHTR coolant channel with the k- $\epsilon$ axisymmetric solver presented in Chapter 5 and the helium property correlations from Chapter 3.

\subsection{Grid Generation}

The axisymmetric representation of the VHTR coolant channel, given Figure 5-1 is a rectangle. Therefore, we use a quadrilateral orthogonal uniform grid to solve for the Navier-Stokes equations. The grid (100x1000) is given Figure 6-3. The grid (100x1000) corresponds to 100 intervals in the radial direction and 1,000 intervals in the axial direction. The red boundary corresponds to the inlet plane, the blue one to the exit plane, the yellow edge to the solid wall and the green one to the symmetry axis.

\subsection{Application of the TVD MacCormack Scheme - Derivation of the Viscous Term}

On the uniform orthogonal grid described in the previous section, the terms $S_{i, j+\frac{1}{2}}^{r}, S_{i, j-\frac{1}{2}}^{r}, S_{i+\frac{1}{2}, j}^{z}$ and $S_{i-\frac{1}{2}, j}^{z}$ are equal to zero which simplifies greatly the predictor, corrector and viscous terms (Equations 5-29 to 5-31).

Computation of the viscous terms require the derivation of velocity and velocity gradients at the cells interfaces $i+\frac{1}{2}, j\left(\right.$ or $\left.i, j+\frac{1}{2}\right)$. We rewrite Equation 5-31 for the uniform, orthogonal mesh as:

$$
\operatorname{Visc}(i, j)=\frac{1}{V o l_{i, j}}\left[S_{i+\frac{1}{2}, j}^{r} F_{i+\frac{1}{2}, j}^{v}+S_{i, j+\frac{1}{2}}^{z} G_{i, j+\frac{1}{2}}^{v}-S_{i-\frac{1}{2}, j}^{r} F_{i-\frac{1}{2}, j}^{v}-S_{i, j-\frac{1}{2}}^{z} G_{i, j-\frac{1}{2}}^{v}\right]
$$

The velocity is evaluated using:

$$
\begin{aligned}
& u_{r}\left(i+\frac{1}{2}, j\right)=\frac{u_{r}(i, j)+u_{r}(i+1, j)}{2} \\
& u_{z}\left(i, j+\frac{1}{2}\right)=\frac{u_{z}(i, j)+u_{z}(i, j+1)}{2}
\end{aligned}
$$

¿From the definition of $F^{v}$ and $G^{v}$ we need to derive the values of $\tau_{r r}$ and $\tau_{r z}$ on the interfaces in the i-direction and the values of $\tau_{z z}$ and $\tau_{z r}$ on the interfaces in the 
j-direction.

$$
\begin{gathered}
\tau_{r r}\left(i+\frac{1}{2}, j\right)=\mu_{t o t}\left[-\frac{4}{3} \frac{\partial u_{r}}{\partial r}-\frac{2}{3}\left(\frac{\partial u_{z}}{\partial z}+\frac{u_{r}}{r}\right)\right]_{i+\frac{1}{2}, j} \\
\tau_{r z}\left(i+\frac{1}{2}, j\right)=\mu_{t o t}\left[\frac{\partial u_{z}}{\partial r}+\frac{\partial u_{r}}{\partial z}\right]_{i+\frac{1}{2}, j} \\
\tau_{z r}\left(i, j+\frac{1}{2}\right)=\mu_{t o t}\left[\frac{\partial u_{z}}{\partial r}+\frac{\partial u_{r}}{\partial z}\right]_{i, j+\frac{1}{2}} \\
\tau_{z z}\left(i, j+\frac{1}{2}\right)=\mu_{t o t}\left[\frac{4}{3} \frac{\partial u_{z}}{\partial z}-\frac{2}{3}\left(\frac{\partial u_{r}}{\partial r}+\frac{u_{r}}{r}\right)\right]_{i, j+\frac{1}{2}}
\end{gathered}
$$

Therefore, for the source term, we need an evaluation of the shear stress tensor component $\tau_{\theta \theta}$ at the center of the cell.

$$
\tau_{\theta \theta}(i, j)=\mu_{t o t}\left[-\frac{8}{3} \frac{u_{r}}{r}-\frac{2}{3} \frac{\partial u_{r}}{\partial r}-\frac{2}{3} \frac{\partial u_{z}}{\partial z}\right]_{i, j}
$$

For the evaluation of the derivatives we use dual cells as seen Figure 6-2, with the vertices located at the centers of gravity of the cells and at the end point of the interfaces. We use

$$
u_{r\left(i+\frac{1}{2}, j+\frac{1}{2}\right)}=\frac{1}{4}\left[u_{r(i, j)}+u_{r(i+1, j)}+u_{r(i, j+1)}+u_{r(i+1, j+1)}\right]
$$

which reduces at the solid walls to $u_{r\left(i+\frac{1}{2}, j+\frac{1}{2}\right)}=0$. Using the nomenclature of Figure 6-2 we get:

$$
\begin{aligned}
\left(\begin{array}{c}
-\frac{\partial u_{r}}{\partial z} \\
\frac{\partial u_{r}}{\partial r}
\end{array}\right)_{i+\frac{1}{2}, j} & =\frac{1}{V o l_{A B C D}}\left(\overrightarrow{A B} \frac{u_{r(i, j)}+u_{r\left(i+\frac{1}{2}, j-\frac{1}{2}\right)}}{2}+\overrightarrow{B C} \frac{u_{r\left(i+\frac{1}{2}, j-\frac{1}{2}\right)}+u_{r(i+1, j}}{2}\right. \\
& \left.+\overrightarrow{C D} \frac{u_{r(i+1, j)}+u_{r\left(i+\frac{1}{2}, j+\frac{1}{2}\right)}}{2}+\overrightarrow{D A} \frac{u_{r\left(i+\frac{1}{2}, j+\frac{1}{2}\right)}+u_{r(i, j)}}{2}\right)
\end{aligned}
$$

We use a similar formulae for the derivatives of $u_{z}$ and $T$. 
When using a uniform normal grid, the surface vectors $\vec{S}$ at the interfaces reduce to:

$$
\left\{\begin{array}{l}
\vec{S}_{i+1 / 2, j}=S_{i+1 / 2, j}^{r} \vec{e}_{r} \\
\vec{S}_{i, j+1 / 2}=S_{i, j+1 / 2}^{z} \vec{e}_{z} \\
\vec{S}_{i-1 / 2, j}=S_{i-1 / 2, j}^{r} \vec{e}_{r} \\
\vec{S}_{i, j-1 / 2}=S_{i, j-1 / 2}^{z} \vec{e}_{z}
\end{array}\right.
$$

where

$$
\left\{\begin{aligned}
S_{i+1 / 2, j}^{r} & =R_{i+\frac{1}{2}} \Delta z \\
S_{i, j+1 / 2}^{z} & =R_{i} \Delta r \\
S_{i-1 / 2, j}^{r} & =-R_{i-\frac{1}{2}} \Delta z \\
S_{i, j-1 / 2}^{z} & =-R_{i} \Delta r
\end{aligned}\right.
$$

\subsection{Simulation Results}

\subsubsection{Validation of the Model}

To validate our model on the coolant channel specific geometry, we perform a code-to-code benchmark: The system is modeled both with FLUENT and our code. Since we do not model temperature and pressure property dependence with FLUENT, the comparison will be done with constant properties, except for density which is modeled with the ideal-gas-law.

To limit the computation time, the benchmark is done on a $10 \mathrm{~cm}$ long section.

Specification of the numerical method is given in Appendix B. It is the same for all the FLUENT studies which are presented through the document.

\subsubsection{Boundaries and characteristics}

- Operating pressure: $P_{\text {operating }}=7 \mathrm{MPa}$

- Properties: They are considered constant and given by Equation $6-1$ and $k=0.304$ $\mathrm{W} / \mathrm{K} . \mathrm{m}$ and $c_{p}=5.1888 \mathrm{~J} / \mathrm{g} . \mathrm{K}$

- Inlet boundary: Typically, the velocity of the fluid in the coolant channel is of the order of a couple of meters per second. Here, we will consider the value of the 
Advanced High Temperature Reactor developed at Oak Ridge National Lab [96]. We consider a purely axial velocity:

$$
u_{z}=2.32 \mathrm{~m} / \mathrm{s} \text {, and } u_{r}=0.0 \mathrm{~m} / \mathrm{s}
$$

. For FLUENT compressible solver, we need to input the inlet mass flow rate, in other words $\dot{m}=0.00231 \mathrm{~kg} / \mathrm{s}$. The inlet temperature for this particular analysis is equal to $400^{\circ} \mathrm{C}$ (i.e. $773 \mathrm{~K}$ ).

- Wall: We consider a constant temperature wall for ease of implementation in both models. The average value is equal to $900^{\circ} \mathrm{C}$.

- Outlet boundary: The outlet pressure is equal to $7 \mathrm{MPa}$.

\subsubsection{Results and simulation comparisons}

The profiles of pressure, temperature and velocity on the axis of the channel section are plotted Figure 6-4 to 6-6. Each figure shows the profiles obtained with FLUENT and our in-house solver.

The pressure drop across a 10-cm section of the coolant channel is equal to $10.24 \mathrm{~Pa}$. Our solver, when implemented with constant properties, gives results of the same order of magnitude as the test-case run with FLUENT,

\subsubsection{On the influence of the real properties simulation}

The same test-section in now run with our in-house solver, accounting for the helium property dependence with pressure and temperature. Figures 6-7 to 6-8 show the profiles of pressure, temperature and velocity respectively, on the axis of the test section for both frozen chemistry and real properties. The differences are not drastic because helium is the gas which behaves closest to the ideal gas law. However, the variable properties should not be neglected during a high temperature gas-cooled reactor study: On a section as small as 10-cm long, the pressure drop calculated with the two solvers differs of $4 \%$. Since the real channel is 7.93-meter long, we can imagine that the difference will grow proportionally. Therefore, it is important to include real property formulation of helium when analyzing the flow in a high temperature gas cooled reactor. 


\subsubsection{Modeling of the VHTR Coolant Channel}

The test case is shown Figure 6-1. At the inlet, we impose a constant axial velocity with corresponds to a Mach number of $0.1: u_{z}=2.32 \mathrm{~m} / \mathrm{s}$, an inlet temperature of 400 K. The wall temperature is linearly dependent with the axial position and varies between $400 \mathrm{~K}$ at the inlet and $1,000 \mathrm{~K}$ at the exit. We impose the exit pressure, $P_{\text {Exit }}=7 \mathrm{MPa}=$ $7.10^{6} \mathrm{~Pa}$. The time step is automatically implemented following relation 5-33. Practically, the time step is typically of the order of $\Delta t=4 \cdot 10^{-8} \mathrm{~s}$. The steady-state is obtained after 12,500 time iterations which corresponds about to 25 min CPU. Figure 6-10 shows the profiles of absolute pressure, temperature of the wall and at the axis, velocity magnitude and density.

The pressure drop across the high temperature gas-cooled reactor core is of the order of 5,500 Pa. The flow, driven by the hot wall, is accelerated and the exit velocity is 1.4 times the inlet velocity. The exit temperature on the axis is about $1,000^{\circ} \mathrm{C}$ which shows that the integrality of the heat generated at the wall is transmitted to the fluid.

\subsection{Conclusion on the Helium Channel Study with Real Property Fine CFD Solver}

The study of the coolant channel in an typical helium-cooled reactor shows the validity of the fine-mesh CFD solver that was developed for real property models. The simulation of a small section with constant property with both FLUENT and our solver shows the accuracy of the CFD solver developed.

The comparison between the frozen chemistry model and the real property simulation shows that even if the profiles calculated are very similar, the numerical differences are significant enough to see benefits of using the variable property solver.

The model of the helium coolant channels gives great confidence in the overall performances of the fine-mesh CFD solver with variable or real fluid properties. 


\section{Exit Condition}

\section{- Pressure}

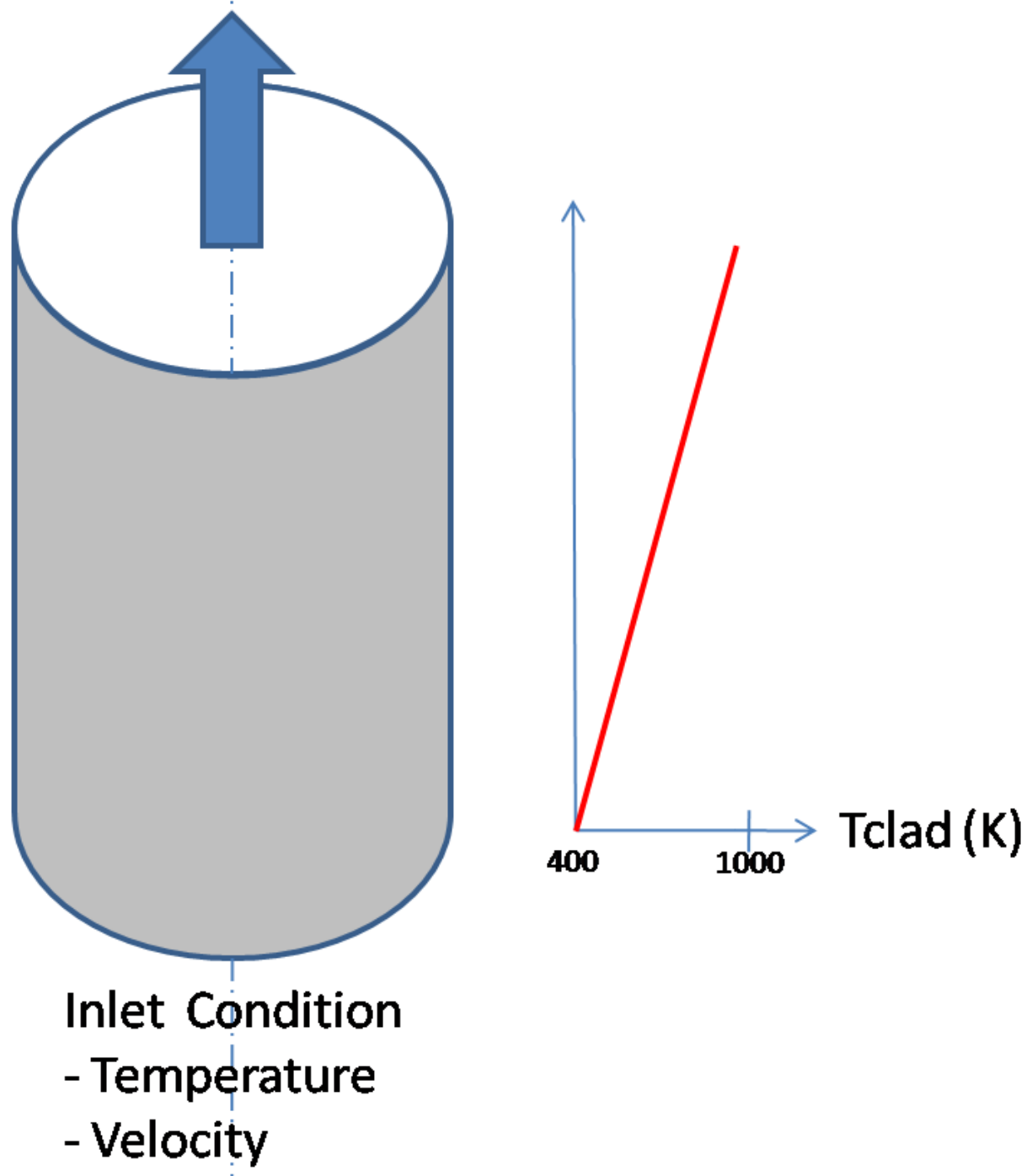

Figure 6-1: VHTR coolant channel 


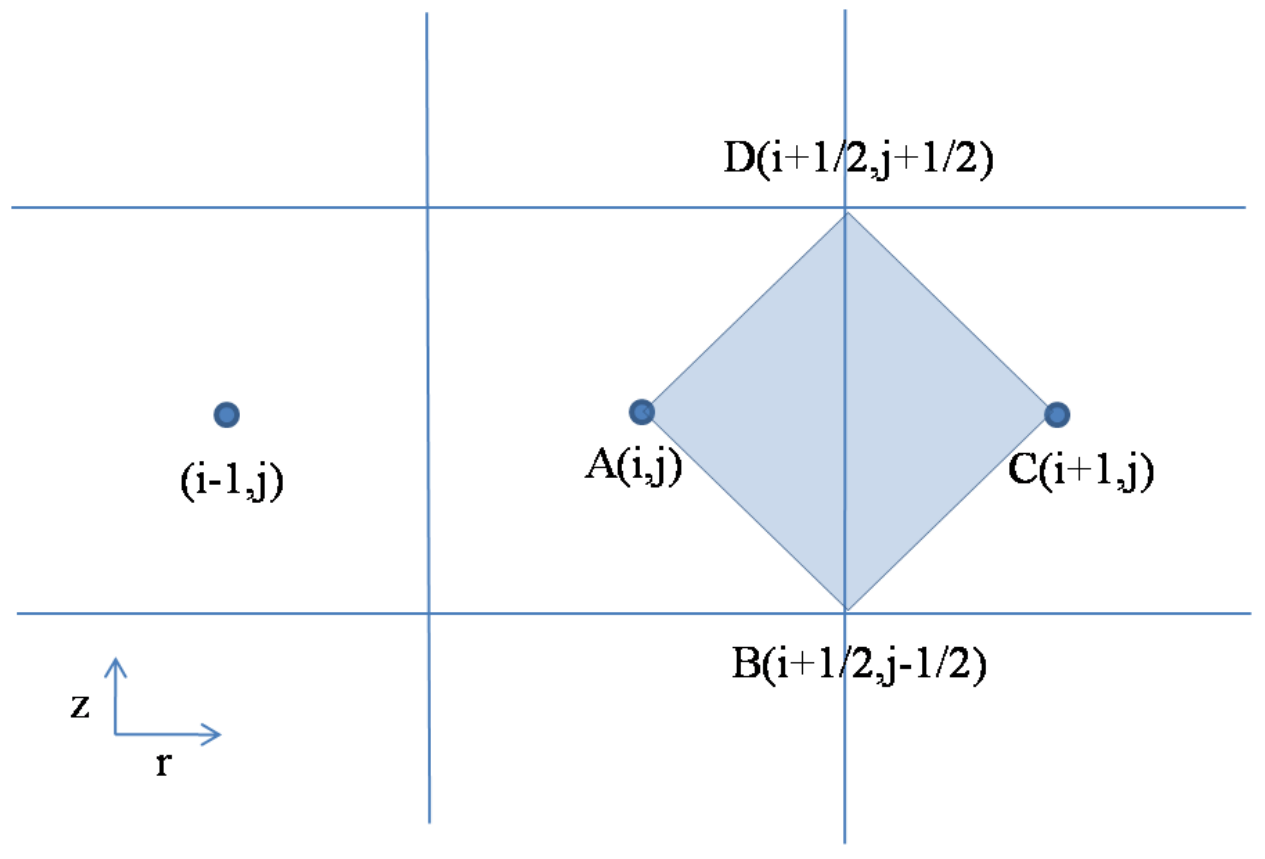

Figure 6-2: Dual cell for the approximation of the derivatives at the cell interface $i+\frac{1}{2}, j$

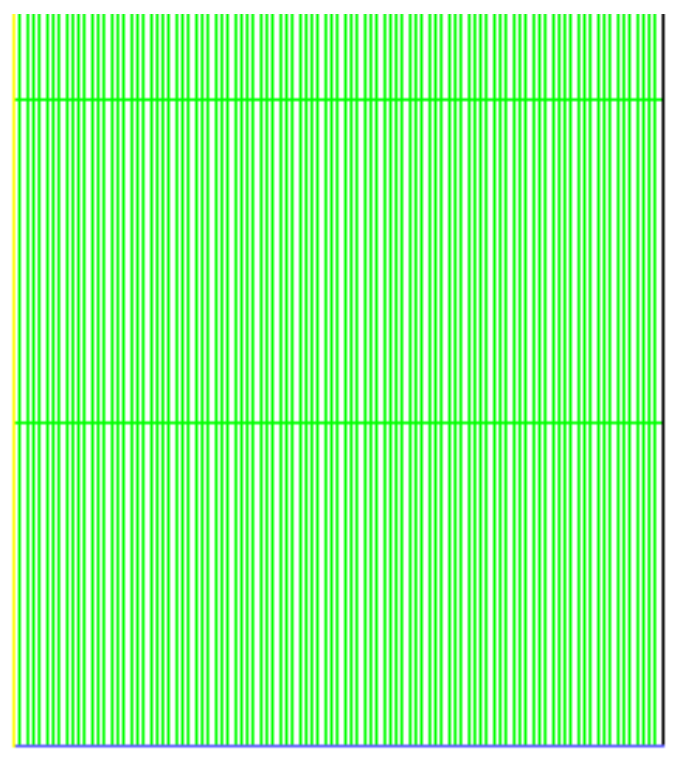

Figure 6-3: Helium channel mesh: 100x1000 quadrilateral grid zoomed at the inlet of the channel. The yellow edge represents the axis, the blue one the inlet and the black one the heated wall. 


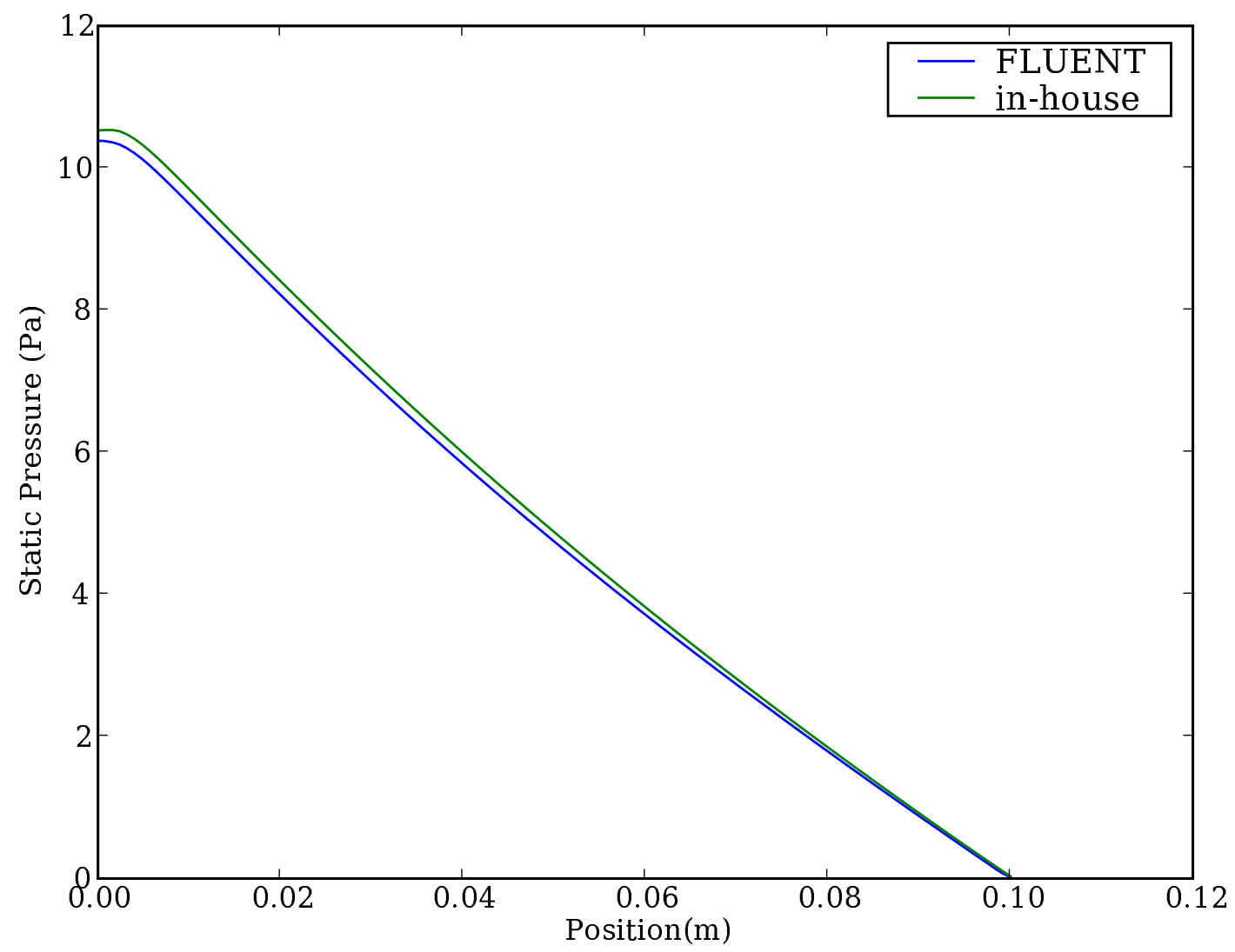

Figure 6-4: Static pressure $(\mathrm{Pa})$ profile on the axis of the test-section. Comparison between FLUENT simulations and our solver. 


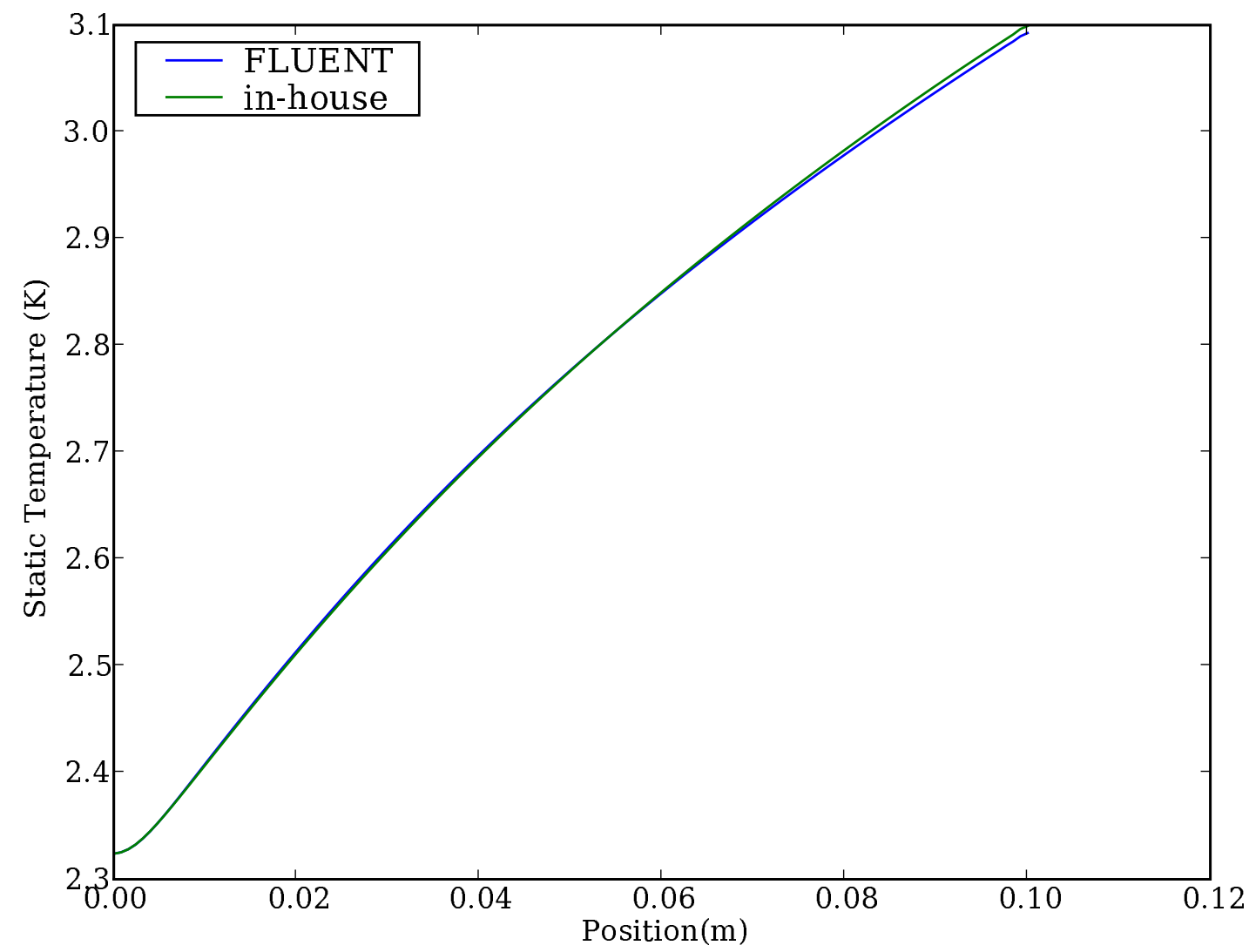

Figure 6-5: Velocity $(\mathrm{m} / \mathrm{s})$ profile on the axis of the test-section. Comparison between FLUENT simulations and our solver. 


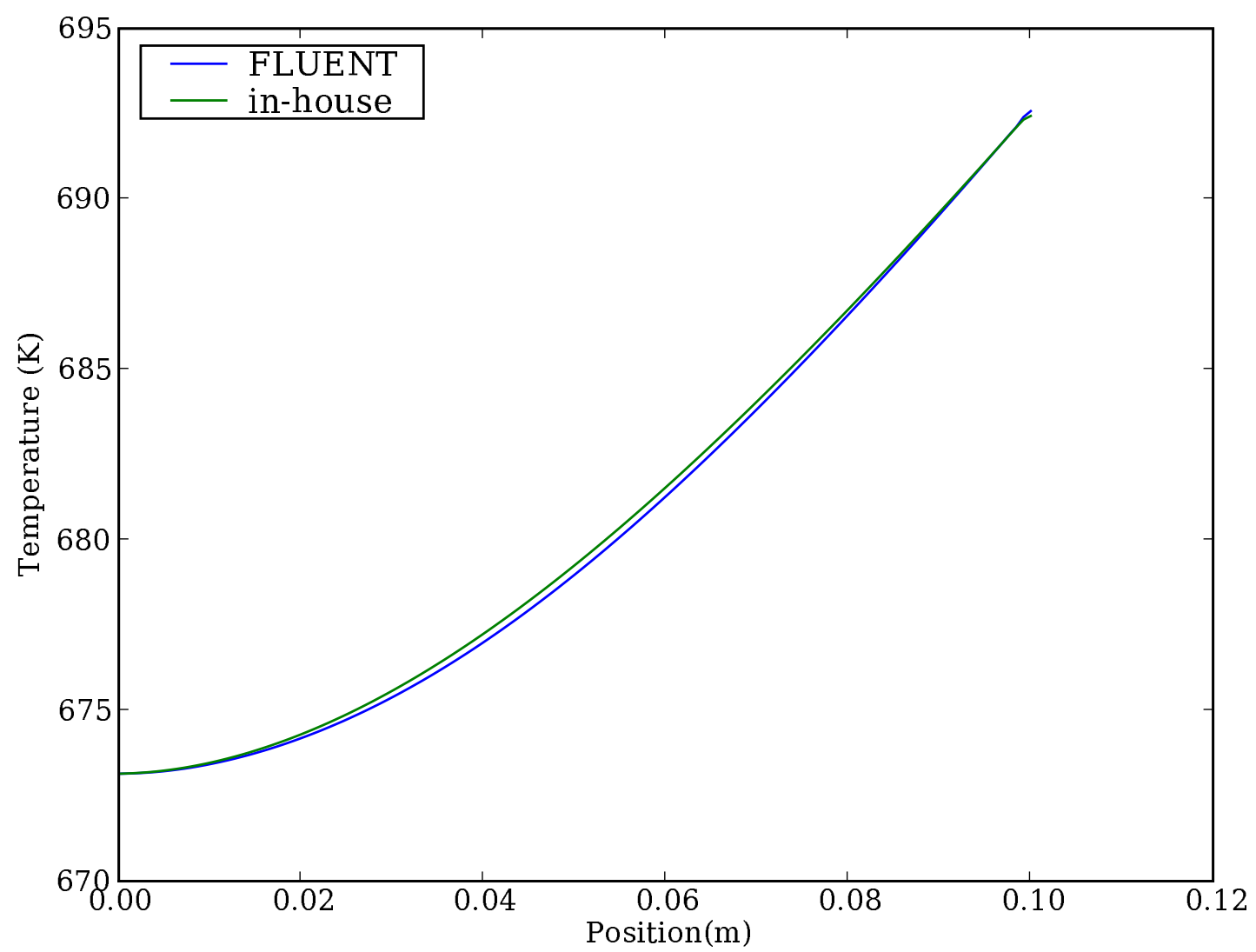

Figure 6-6: Temperature (K) profile on the axis of the test-section. Comparison between FLUENT simulations and our solver. 


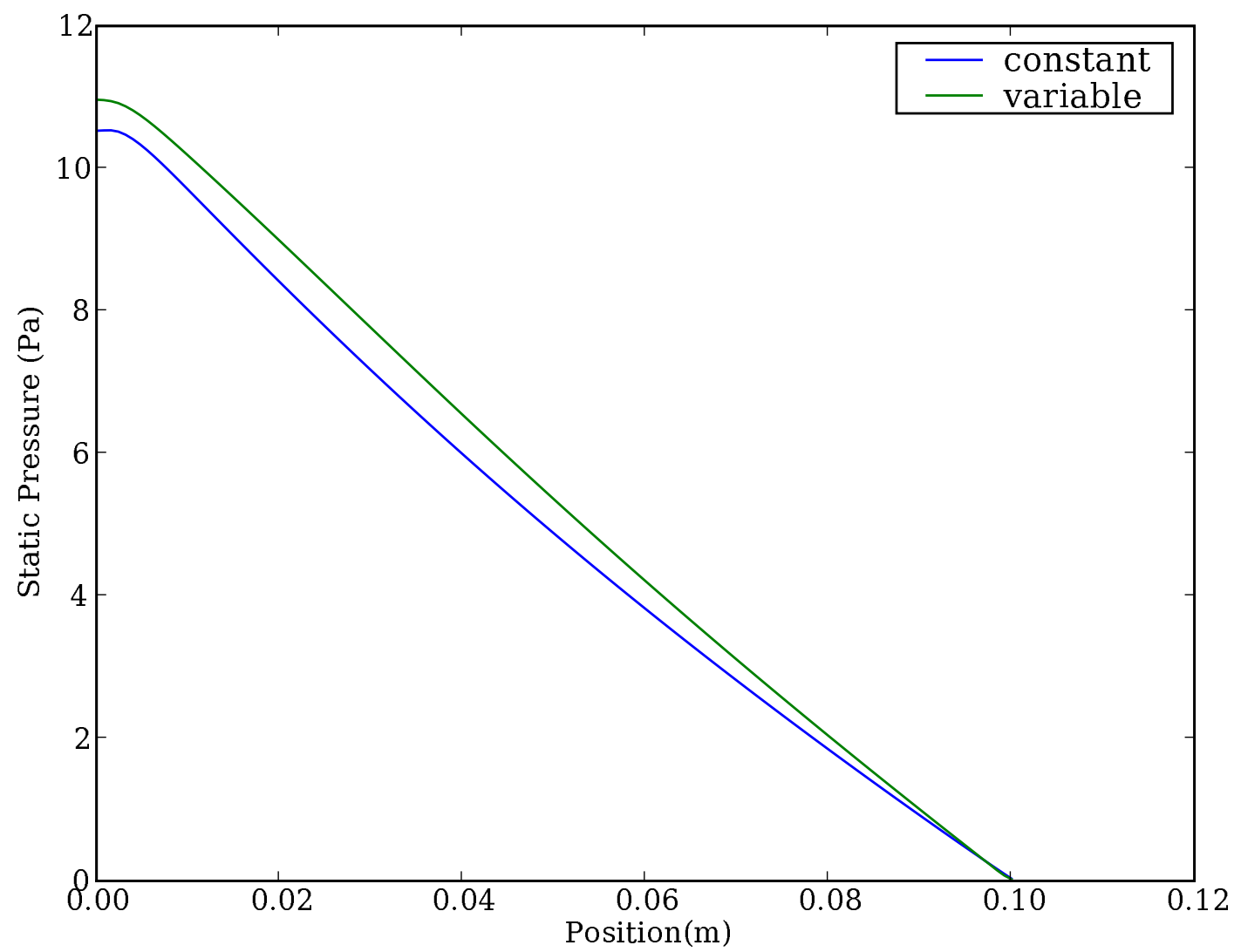

Figure 6-7: Static pressure $(\mathrm{Pa})$ profile on the axis of the test-section. Comparison between constant and real property formulations. 


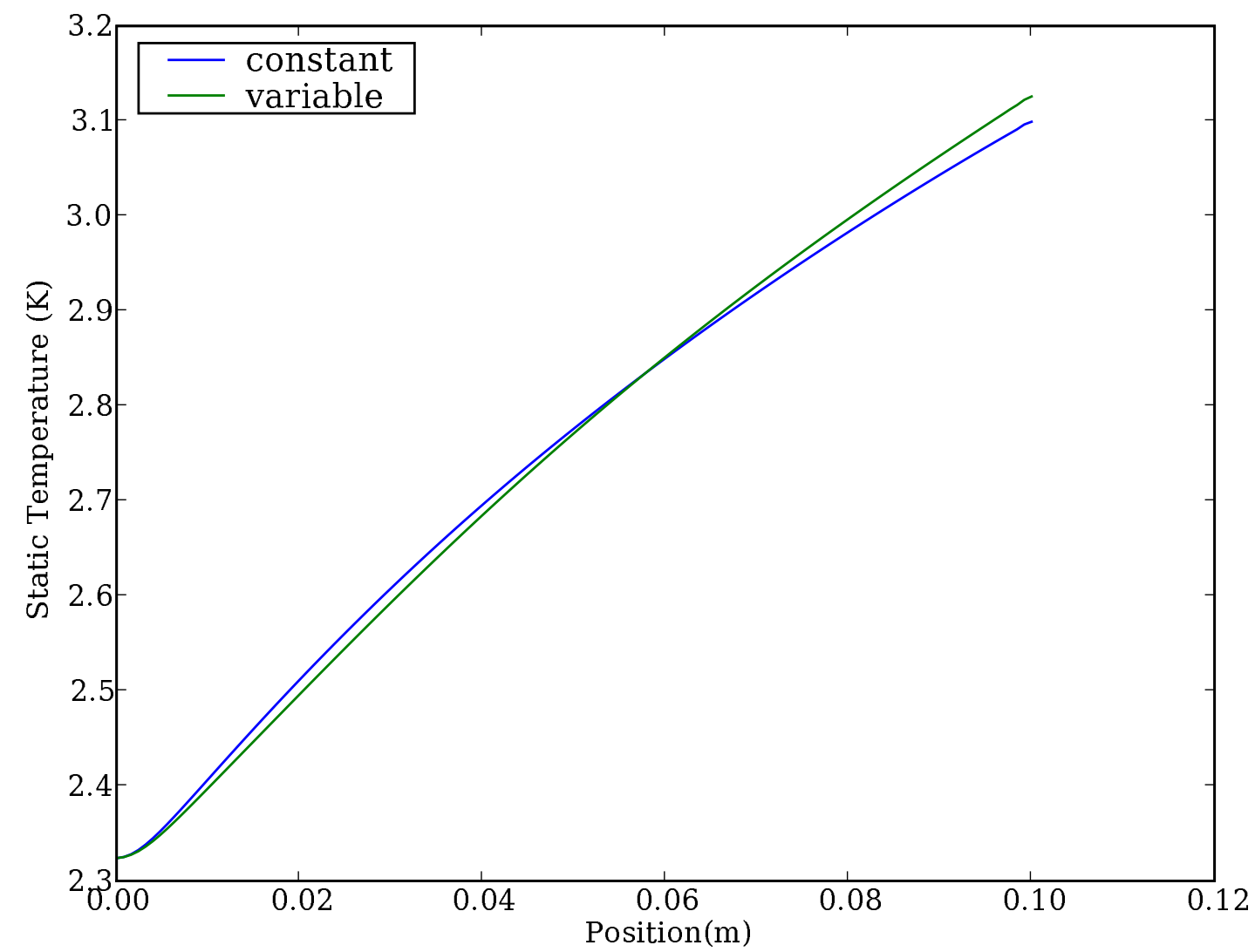

Figure 6-8: Velocity $(\mathrm{m} / \mathrm{s})$ profile on the axis of the test-section. Comparison between constant and real property formulations. 


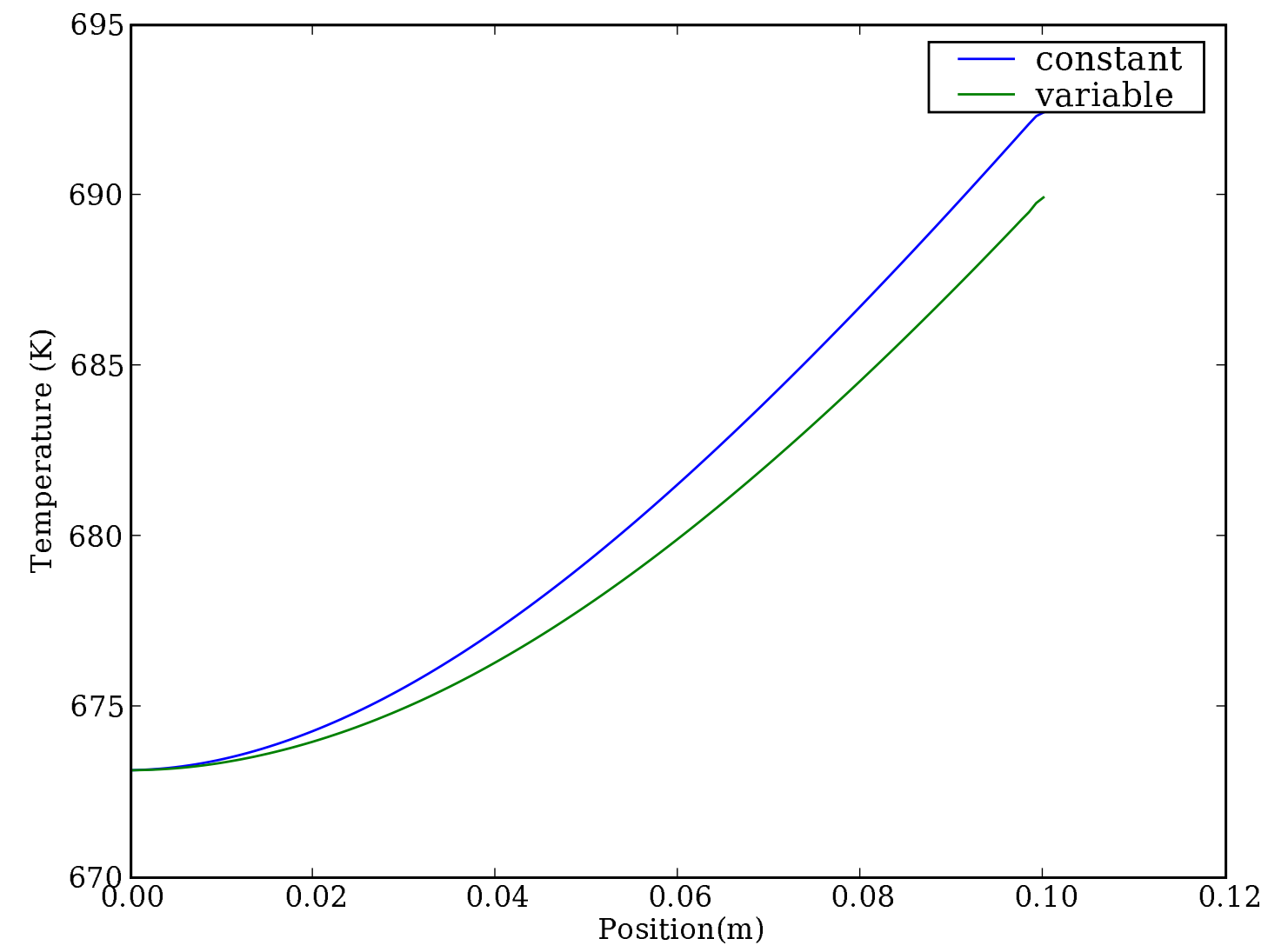

Figure 6-9: Temperature (K) profile on the axis of the test-section.Comparison between constant and real property formulations. 

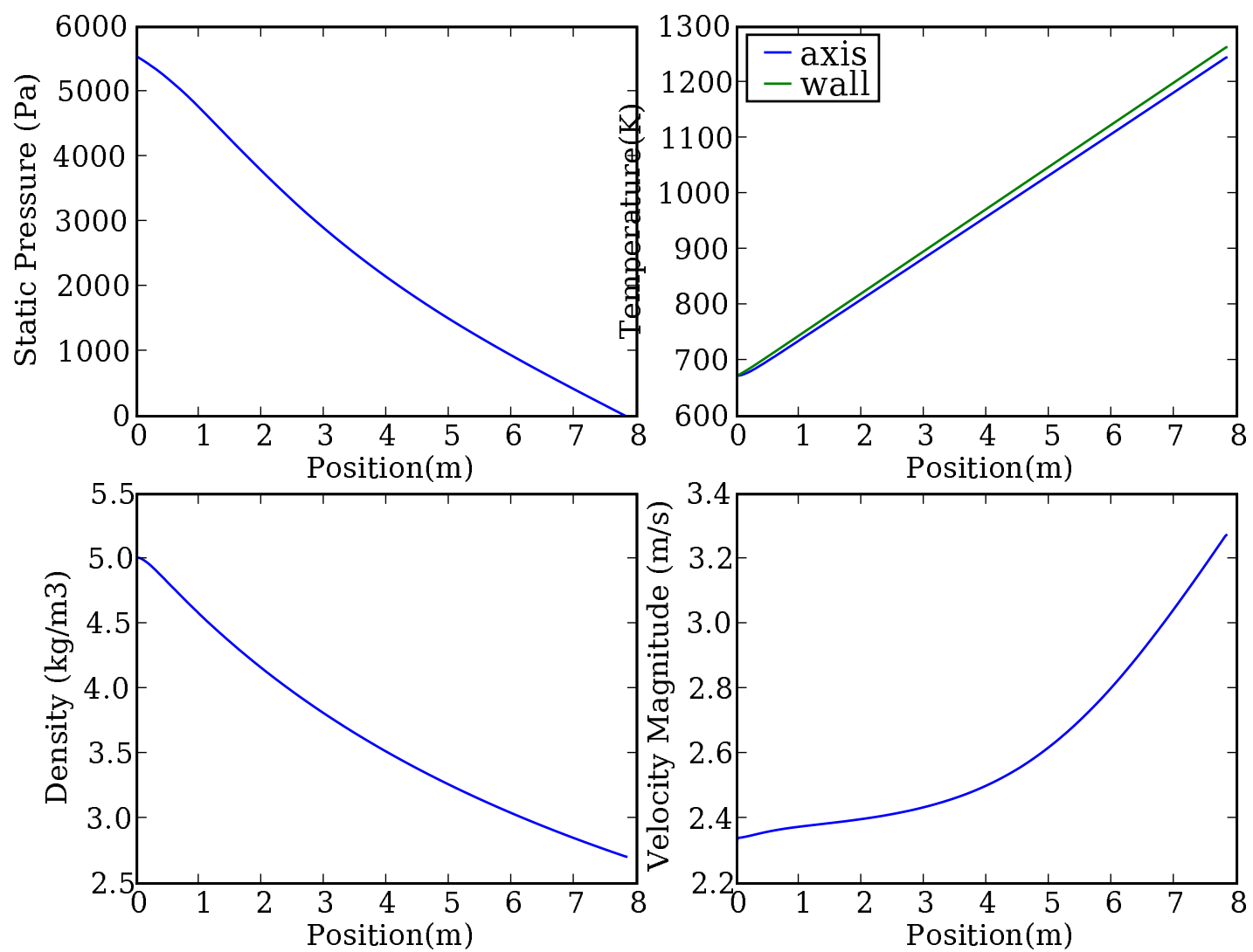

Figure 6-10: Property profiles across the helium cooling channel. 


\section{CHAPTER 7 \\ CFD STUDY OF THE LOW-PRESSURE THRUST CHAMBER}

\subsection{Presentation of the Low-Pressure Thrust Chamber}

The core of the nuclear propulsion heats up hydrogen gas to temperatures ranging from $2,800 \mathrm{~K}$ to $3,250 \mathrm{~K}$ depending on the design. The hot hydrogen then enters a converging-diverging nozzle or thrust chamber where the gas is accelerated and provides thrust to the system. In typical NTP designs, the thrust chamber inlet pressure is of the order of $1850 \mathrm{psi}$, or $12 \mathrm{MPa}$. Table 7-1 shows the main thermal characteristics of hydrogen at the inlet of the thrust chamber.

If the pressure of the gas is decreased by 3 orders of magnitude ( 0.1 bar for example), hydrogen at the exit of the core is dissociated. Its properties are also compiled in Table 7-1 and a schematics of the system is shown Figure 7-1 For the dissociated gas the heat capacity is eight times greater than the one of $\mathrm{H}_{2}$. Also, the specific ratio is much smaller. We saw Figure 5-9 that lower specific heat ratios lead to greater velocities. Therefore, we expect the velocities on the thrust chamber exhaust plane to be greater for hydrogen at low pressure. Moreover, as the gas travels through the chamber, its temperature decreases. Hydrogen at the inlet of the thrust chamber is highly dissociated (up to $30 \%$ ) and recombines as it flows through the nozzle. The energy balance is transferred under the form of kinetic energy, which helps increase the exhaust velocity and the system ISP.

To validate our assumption, we first use a quasi one-dimensional approach to derive the basics geometrical information on the nozzle (throat dimension) and the nozzle characteristics (exhaust velocity). Then, we present the method for the nozzle contour design. Once all the geometrical parameters are derived, we present two studies: the first one is a FLUENT analysis of the nozzle with frozen chemistry, the second analysis will use our solver. 
Table 7-1: Hydrogen thermal properties (heat capacity,specific heat ratio and thermal conductivity) for diverse sets of operating conditions

\begin{tabular}{lllll}
\hline Pressure (bars) & Temperature $(\mathrm{K})$ & $c_{P}(\mathrm{~kJ} / \mathrm{kg} . \mathrm{K})$ & $\gamma$ & $\mathrm{k}(\mathrm{W} / \mathrm{m} . \mathrm{K})$ \\
\hline 120 & 3,000 & 23.48 & 1.250 & 1.696 \\
0.1 & 3,000 & 179.5 & 1.131 & 15.31 \\
\hline
\end{tabular}

\subsection{Quasi-one-dimension Isentropic Analysis of the Low Thrust Chamber}

First we derive the throat radius and the exit Mach number with the isentropic relation [97]:

$$
\frac{A}{A^{*}}=\frac{1}{M}\left[\left(\frac{2}{\gamma-1}\right)\left(1+\frac{\gamma-1}{2} M^{2}\right)\right]^{\frac{\gamma+1}{2(\gamma-1)}}
$$

where, $A$ is the area of the nozzle at a given point and $M$ the Mach number of the flow at this point. $A^{*}$ is the area at the throat, where $M=1$.

The relation is accurate for one-dimensional steady flow where body forces and gravitational effects are negligible, for a frictionless ideal gas with constant specific heat and in the case of an adiabatic flow with no shear and shaft work. Some of the assumptions such as constant specific heat are not verified. However, in this first study we look for an order of magnitude for the nozzle performances. Therefore, we will assume that all the conditions of the isentropic study are verified.

The inlet Mach number in the system is equal to 0.3 and the inlet radius of the thrust chamber is equal to $30 \mathrm{~cm}$. Hence, the throat radius is:

$$
r^{*}=20.77 \mathrm{~cm}
$$

We now calculate the Mach number on the exit plane for an exit radius of $1 \mathrm{~m}$. We solve Equation $7-1$ for the ratio $\frac{A}{A^{*}}=23.19$. We get:

$$
M_{\text {exit }}=3.569
$$


To derive the exhaust velocity knowing $M_{\text {exit }}$ we need the exhaust speed of sound $a_{\text {exit }}$ :

$$
M_{\text {exit }}=\frac{v_{\text {exhaust }}}{a_{\text {exit }}}
$$

The speed of sound for an ideal gas is given by:

$$
a_{e x i t}=\sqrt{\gamma R T_{\text {exit }}}
$$

Therefore, we need to derive the exit temperature. To do so, we use the Rayleigh relations. The Rayleigh relations are accurate here, since they are aimed at modeling flows with heat exchanges where the viscosity remains negligible. The ratio of temperature $T$ at the location where the Mach number is equal to $M$ over the temperature at the throat $T^{*}$ is given by:

$$
\frac{T}{T^{*}}=\frac{(1+\gamma)^{2} M^{2}}{\left(1+\gamma M^{2}\right)^{2}}
$$

We get:

$$
\left(\frac{T}{T^{*}}\right)_{M=0.3}=0.336676 \text { and }\left(\frac{T}{T^{*}}\right)_{M=3.568}=0.243820
$$

Then,

$$
T_{\text {exit }}=\frac{\left(\frac{T}{T^{*}}\right)_{M=3.568}}{\left(\frac{T}{T^{*}}\right)_{M=0.3}} T_{\text {inlet }}=2,172.65 \mathrm{~K}
$$

We approximate the molecular weight of the hydrogen of the flow to the hydrogen molecular weight at $3,000 \mathrm{~K}$ and 0.1 bar: $M=1.622 \mathrm{~g} / \mathrm{mol}$.

Finally, the speed of sound at the exit is $a_{e x i t}=\sqrt{\gamma R T_{\text {exit }}}=3549 \mathrm{~m} / \mathrm{s}$.

Using the quasi-one-dimensional relation for isentropic flow, and the Rayleigh relations to account for heat transfers, we expect the gas velocity at the exhaust of the thrust chamber to be equal to $\mathbf{1 2 , 6 6 3} \mathbf{m} / \mathrm{s}$ for an inviscid flow. 
As the Mach number of a flow increases, the effects of viscosity cannot be neglected anymore. We can account for viscous effects when considering a Fanno flow. The ratio of temperatures becomes:

$$
\frac{T}{T^{*}}=\frac{\gamma+1}{2+(\gamma-1) M^{2}}
$$

Now,

$$
\left(\frac{T}{T^{*}}\right)_{M=0.3}=1.05926 \text { and }\left(\frac{T}{T^{*}}\right)_{M=3.568}=0.581016
$$

Then,

$$
T_{\text {exit }}=1645.53 \mathrm{~K}
$$

When considering an adiabatic viscous flow the exhaust velocity becomes:

$$
\mathbf{v}_{\text {exit }}=11,020 \mathrm{~m} / \mathrm{s}
$$

Depending on the approach when leading the quasi-one-dimensional we get different exhaust velocity values:

- Inviscid adiabatic flow $v_{\text {exit }}=12,663 \mathrm{~m} / \mathrm{s}$

- Viscous adiabatic flow $v_{\text {exit }}=11,020 \mathrm{~m} / \mathrm{s}$

In both cases, we derive an ISP value greater than the ISP for thrust chamber at high pressure.

For the real low-pressure thrust chamber, the assumptions to use Reighleigh flow, Fanno flow and isentropic flow relations are not all verified. To get a more accurate description of the nozzle flow we need to model the thrust chamber with a two-dimensional solver.

\subsection{Two-Dimensional Contour Profile of the Thrust Chamber Walls}

To design the contours of the low pressure nozzle the method of Rao [98] extended to real-gas is chosen, coupled to a CFD-based optimization procedure elaborated by Korte [99]. The converging diverging nozzle is divided into a subsonic-throat region, a transonic region and a supersonic region. The specific heat ratio at the inlet of the nozzle 
is equal to 1.131 . The throat radius is estimated for a Mach number of 0.3 by using the isentropic relationships for converging nozzles. The throat radius is equal to $15.9 \mathrm{~cm}$. The minimum area of the nozzle is located at $z=0$.

- Converging region:

In that portion the flow remains subsonic. The nozzle radius at a position $\mathrm{z}$ is given by:

$$
y=r^{*}\left(C_{1}\left(1-e^{-\frac{\left(z / r^{*}\right)^{2}}{2 C_{1} R_{c}}}\right)-1\right)
$$

where,

$$
C_{1}=R_{c} \tan ^{2}\left(\theta_{a}\right) * e
$$

- Transonic region:

The transonic region is described by a circle of radius $R_{c} r^{*}$

- Supersonic region:

The supersonic contour is described by a contoured nozzle geometry-cubic polynomial [100]. The nozzle radius $y$ at an axial position $z$ is given by:

$$
y=y_{0}+y_{0}^{\prime}\left(z-z_{0}\right)+c\left(z-z_{0}\right)^{2}+d\left(z-z_{0}\right)^{3}
$$

with

$$
\left\{\begin{array}{l}
c=\frac{3\left(y_{L}-y_{0}-y_{0}^{\prime} L\right)}{L^{2}}-\frac{\left(y_{L} \prime-y_{0} \prime\right)}{L} \\
d=\frac{\left(y_{L}^{\prime}-y_{0}^{\prime}\right)}{L^{2}}-\frac{2\left(y_{L}-y_{0}-y_{0}^{\prime} L\right)}{L^{3}}
\end{array}\right.
$$

To design the optimum converging-diverging nozzle, five parameters have to be determined:

- subsonic approach angle $\theta_{a}$

- nozzle inflection angle $\theta$

- throat radius of curvature $R_{c}$

- slope at the exit of the chamber $y_{L}^{\prime}$

- length of the diverging portion $L$

In the following analysis, we make the assumption that the slope at the exit of the nozzle is equal to zero: $y_{L}^{\prime}=0$. The best nozzle shape is determined by looking at the 
performances associated to each design. Uniform velocity profile and maximum velocity on the exit plane are a proof of optimum performances.

\subsection{Grid Generation}

We generate a structured grid: the interfaces in the axial direction are parallel to the inlet and exhaust plane. The radial interfaces are aligned with the flow path. In the area of the throat, the density of cells is greatly increased.

For the coarse mesh shown Figure 7-2, the length of the meshes is equal to $10 \%$ of the nozzle radius at that location. We also create a boundary layer in the area of the wall to resolve properly the boundary layer. The grid we show Figure 7-2 is very coarse and does not resolve the boundary layer. Therefore the nodes are equidistant in the radial direction.

\subsection{Low-Pressure Thrust Chamber Analysis using FLUENT and Frozen Chemistry}

This section aims at having a preliminary look at the numerics choices possible for the computational case. Once the suitable model is chosen, we do a sensitivity study on the design parameters of the nozzle contours.

\subsubsection{FLUENT}

FLUENT is a commercial CFD code which uses a finite volume method. A pre-processor, GAMBIT allows mesh generation. All modeling capabilities are accessible through a GUI. The versions we use are GAMBIT 2.3.16 and FLUENT 6.3.26. Modeling details and definitions are given in Appendix B.

\subsubsection{Numerical Procedure}

Description of hydrogen properties with temperature and pressure are complex to model in a simple fashion. Therefore, we present here an analysis of the low-pressure thrust chamber with frozen chemistry. Frozen chemistry consists in keeping all hydrogen properties constant and equal to the inlet properties, except for density which is modeled with the ideal-gas law. 
The design base for this study is the nozzle with:

$$
\left\{\begin{array}{l}
\theta_{a}=20^{\circ} \\
R_{c}=2 \\
r_{\text {exit }}=1 \mathrm{~m} \\
\theta=40 \\
L=3 \mathrm{~m} \\
y_{L}^{\prime}=0
\end{array}\right.
$$

Our interest in this section is not the nozzle performance but the determination of the most accurate numerical methods and models. Therefore, we will use the geometrical parameters $7-5$, regardless their influence on the flow map.

The nozzle of interest is the low pressure thrust chamber at the exit of the SLHC nuclear core, i.e.

$$
\left\{\begin{array}{l}
r_{i}=30 \mathrm{~cm} \\
M_{i}=0.3 \\
T_{i}=3,000 \mathrm{~K} \\
P_{i}=0.1 \text { bars }
\end{array}\right.
$$

In reality, the gas exits the nozzle and is released in space, where the surrounding pressure is close to 0 . Hence, the exit pressure in the simulation is set to $10 \mathrm{~Pa}$. In the following study, we will limit our model to an adiabatic system with isothermal walls.

The fluid used in the system is hydrogen as described in Chapter 4. Since FLUENT does not enable modeling of the fluid properties as a function of both pressure and temperature, we will consider frozen chemistry: we use the ideal-gas law formulation of density and all the other properties are constant and equal to the properties of hydrogen 
at the inlet of the system:

$$
\left\{\begin{aligned}
m & =1.622 \mathrm{~g} / \mathrm{mol} \\
c_{p} & =179,500 \mathrm{~J} /(\mathrm{kg} . \mathrm{K}) \\
\mu & =4.47 * 10^{-5} \mathrm{~Pa} . \mathrm{S} \\
k & =15.31 \mathrm{~W} /(\mathrm{m} . \mathrm{K})
\end{aligned}\right.
$$

To capture properly the pressure in the supersonic fluid, we use the third order MUSCL scheme on density, momentum components and energy. We also use a second order upwind scheme on pressure and turbulence components.

In the case of the system of Navier-Stokes equations we use the Spalart-Allmaras turbulence model.

\subsubsection{Analysis results}

\subsubsection{Investigation of the nozzle representation}

FLUENT allows for three type of representations of any fluid system: 2D, 2D

axisymmetric and 3D. A three-dimensional representation always gives the best simulations, however it is computationally expansive: 3D models require a lot of computational cells. In systems which are symmetric with respect to an axis, such as a tube, or here the thrust chamber, a 2D axisymmetric representation is often sufficient. This is true provided there are no three-dimensional effects occurring in the flow. The two-dimensional representation of a system is the simplest one, but one need to be careful when using this representation: in axisymmetrical geometries, a two-dimensional representation neglects some effects due to symmetry of rotation.

We investigate the accuracy of each of the representations offered by FLUENT. For a given set of nozzle design parameter we create one two-dimensional model, one axisymmetric model and on three-dimensional model with the same numerical cell density. The goal is to see if a two-dimensional model of the thrust chamber would be accurate enough. Figure 7-3 shows the profile of Mach number on the axis of each of the models. It shows clearly that the two-dimensional model under-estimates the exhaust Mach number 
and is not adapted for the thrust chamber study. Three-dimensional and axisymmetric models show very similar profiles. Therefore, the axisymmetric solver is chosen for this study. This preliminary analysis confirms our choice to develop a single axisymmetric solver for the thrust chamber analysis.

\subsubsection{Which governing equation to use?}

Flows are considered inviscid when the viscous or friction forces are small in comparison to the inertial force. The Reynolds number quantifies the ratio of the inertial forces over the viscous forces. Therefore, when the Reynolds number is large, the flow can be considered inviscid. In the case of the low-pressure nozzle, the inlet Reynolds number is equal to $1.7 * 10^{4}$ and the Navier-Stokes equations can be simplified to the Euler equations.

FLUENT enables modeling of inviscid flow by selecting 'inviscid' in the turbulence panel. Besides the governing equation, the principal difference between inviscid and viscous flow lays in the wall boundary condition treatment. For an inviscid flow, the slip condition applies: Since there is no viscosity the fluid tangential velocity at the wall is not equal to zero. On the opposite, in the case of a viscous fluid, the velocity at the wall is equal to the velocity of the wall itself: the relative velocity between the wall and the fluid adjacent is equal to zero.

Figure 7-4 shows the Mach number profile on the axis of the thrust chamber for both Euler and Navier-Stokes solvers. The profiles are similar, except at the exit of the nozzle. This is consistent with the results we obtained in the quasi-one-dimensional analysis: the exhaust velocity is slightly higher(14\%) for an inviscid flow than for a viscous flow.

Therefore, in a first approach, the Euler solver could be used to model the flow in the low-pressure thrust chamber. However, as the hydrogen travels trough the chamber, the viscous effects cannot be neglected anymore. Thus, the Navier-Stokes solvers are more adapted to model the flow in the low-pressure thrust chamber. 


\subsubsection{Investigation of the turbulence model}

The literature review presented in Chapter 3 showed the adequacy of the Spalart-Allmaras turbulence in the particular case of the thrust chamber modeling. FLUENT allows us to compare easily the performances of several RANS turbulence models and assess their performances. The following models are investigated: Spalart-Allmaras, standard k- $\epsilon$, realizable k- $\epsilon$ with enhanced wall-treatment, $\mathrm{k}-\omega$ and RSM. The profiles of pressure on the walls of the nozzle (not shown) are very similar for all the models. The profiles of Mach number on the nozzle axis is given for each turbulence model Figure 7-5. We notice that the $\mathrm{k}-\epsilon$ model with enhanced wall treatment gives the worst results. Because RSM is the most advanced turbulence model within the RANS technique, we can consider the profiles for this model as being the reference: RSM models all the fluctuating products within the RANS approach by soling a transport equation, and therefore, modeling of the turbulent effects is much more restricted than in the other models. The Spalart-Allmaras model shows very good agreement with the RSM predictions. This analysis confirms the good use of the Spalart-Allmaras model in the case of the nozzle modeling.

\subsubsection{Sensitivity study}

As the number of computational cells increase towards infinity the numerical solution becomes more and more similar to the real flow. There is a threshold where the number of cells is large enough that if the number of cells is increased, the solution remains unchanged. The goal of the sensitivity study is to derive the minimum number of computational cells required to obtain a solution as close as possible from the real flow field. To allow for faster computations, the grid is built such as the edges of the meshes are parallel to the flow direction.

Six meshes are generated with GAMBIT, the grid processor associated to FLUENT. The following grids are investigated:

- G1: 6 x 51 (306)computational nodes.

- G2: 10 x 51 (510) computational nodes. 
- G3: 19 x $101(1,919)$ computational nodes.

- G4: 28 x $151(4,228)$ computational nodes.

- G5: 37 x $201(7,437)$ computational nodes.

- G6: 46 x $251(11,546)$ computational nodes.

Results of the analysis are presented Figure 7-6: the relative differences between the maximum Mach number compiled with a given grid and the finest grid are plotted as a function of the number of computational nodes for the given grid. As the number of mesh points increase, the difference becomes smaller and smaller, and tends towards zero. The variation as the number of nodes increases follows an exponential decay curve. Table 7-2 compiles the $\mathrm{CPU}$ time and number of iteration required to reach convergence for grids G1 to G5.

We need to make a compromise between accuracy and CPU time. From Figure 7-6 and Table7-2 we can interfere that grids G2 is well adapted to get fast results. We might want to use grid G3 in cases where we want to increase accuracy of the simulation.

\subsubsection{Design Parameters Sensitivity Study}

All the previous analyzes considered a single nozzle design, characterized by the design parameters $7-5$. Before we start our own CFD study of the nozzle, we want to investigate which combination of the design parameters lead to the best nozzle performance. We use the frozen chemistry model above to investigate the best design. The performance criteria are:

- maximum velocity in the nozzle should be localized on the exhaust plane

- the best design exhibits the highest exhaust velocity

- the profile of velocity on the exhaust plane should be as uniform as possible Bullets 1 and 3 ensure that the gas is properly expanded in the nozzle and bullet 2 that the thrust chamber exhibit great ISP values.

We realize an axiomatic design theory, i.e. we investigate the different design parameters independently. These parameters are $R_{e x i t}, \theta_{a}, \theta, R_{c}$ and $L$. Through this 
analysis, the slope of the nozzle at the exit is considered equal to zero: $y_{L}^{\prime}=0$. This is particularly true in all the designs we reviewed. This is a parameter which value should be addressed in a more detailed analysis. Table 7-3 lists the different cases investigated and the design parameters associated. A ' $\mathrm{x}$ ' in the last column indicates that the case is geometrically impossible to create, and a 'o' means that the gas is not properly expanded in the chamber. As a rule of thumb, the gas usually does not expand in the chamber when the diverging nozzle is either too short or too long. The table also lists the maximum velocity in each case.

Figure 7-7 shows the influence of an isolated parameter on the nozzle performances while all other parameters remain constant.

Logically, high throat to exit ratios $\left(A / A^{*}\right)$ lead to higher velocities and Mach numbers on the exhaust plane. An exit radius of $1.5 \mathrm{~m}$ corresponds to a ratio of about 100. This is the type of ratio we want to investigate. The thorough investigation exhibits a set of parameters which lead to highest performances of the nozzle with $A / A^{*}=100$ :

$$
\left\{\begin{array}{l}
R_{c}=1 \\
\theta_{a}=35^{\circ} \\
\theta=40^{\circ} \\
L=3.3 \mathrm{~m}
\end{array}\right.
$$

The optimum design corresponds to 'Grid45' and leads to velocities of $14,358 \mathrm{~m} / \mathrm{s}$, which corresponds to Isp of $1,464 \mathrm{~s}^{-1}$. The Isp is also a measure of the available working fluid over the molecular weight of that fluid. Since hydrogen properties are accounted for with frozen chemistry, the molecular weight of hydrogen is equal to 1.662 in the exhaust plane. When accounting for the real properties, there is hydrogen recombination which occurs in the chamber. Therefore, the actual molecular weight of the exhausting gas might be slightly greater and the study with FLUENT might overpredict the real value of the Isp. 


\subsubsection{Influence of the thermal formulation}

In all the cases investigated so far, the walls were considered adiabatic. In the real system, the cold hydrogen which exits the tank flows on the walls of the thrust chamber. A simple run with NuRok shows that the wall temperature can be modeled with a constant equal to $1,500 \mathrm{~K}$. The changes in the exit velocity value is negligible $(0.3 \%)$ when we change the adiabatic into constant temperature walls. Figure 7-8 shows the exhaust velocity profiles for the two formulations on the wall condition.

\subsubsection{Influence of the nozzle slope at the exit of the system}

When we look at the Mach number profile on the axis of the nozzle in case $7-8$, we notice that the maximum Mach number is not located on the exhaust plane but a few centimeter before. This means that the flow is not optimally expanded. There are two reasons why the gas in not expanding properly: the length of the converging nozzle could be either too long or too short, or the slope of the wall at the exit could be not adapted to the problem.

\subsection{Our in-house solver}

The grid is generated as a part of the solver. This is done with several objectives: First, it enables the user to compare the performances of different designs by inputting design parameters. Once a set of optimum design parameters is determined, the geometry of the nozzle depends on the inlet velocity. The reader should have in mind that the solver is developed in order to couple it with the coarse solver NuRok: as the solution is iterated, diverse nozzle inlet velocities might be derived. The mesh generator being part of the solver allows to consider any values of inlet velocities.

Because of the large variation in the values of the density and heat capacity with a small pressure or temperature gradient, the property loop has not been converged yet.

In the particular case of the low pressure thrust chamber, more investigation would be required to get convergence of the property loop in particular and the solver in general. One of the solution could be to start the first time step with a coarser definition of the 
properties: for example, as a first approximation, use a simple linear function of the properties as a function of temperature. As the solver iterates, a more refined definition of the properties could be implemented. 
Table 7-2: CPU time (s) and number of iteration to reach convergence of the steady-state for the four different grids

\begin{tabular}{ccccc}
\hline Grid & G2 & G3 & G4 & G5 \\
\hline CPU(s) & $2: 51: 04$ & $5: 24: 09$ & $7: 08: 16$ & $10: 03: 35$ \\
Iteration Nb & 72 & 107 & 124 & 146 \\
Time per iteration & $0: 02: 23$ & $0: 03: 02$ & $0: 03: 27$ & $0: 04: 08$ \\
\hline
\end{tabular}

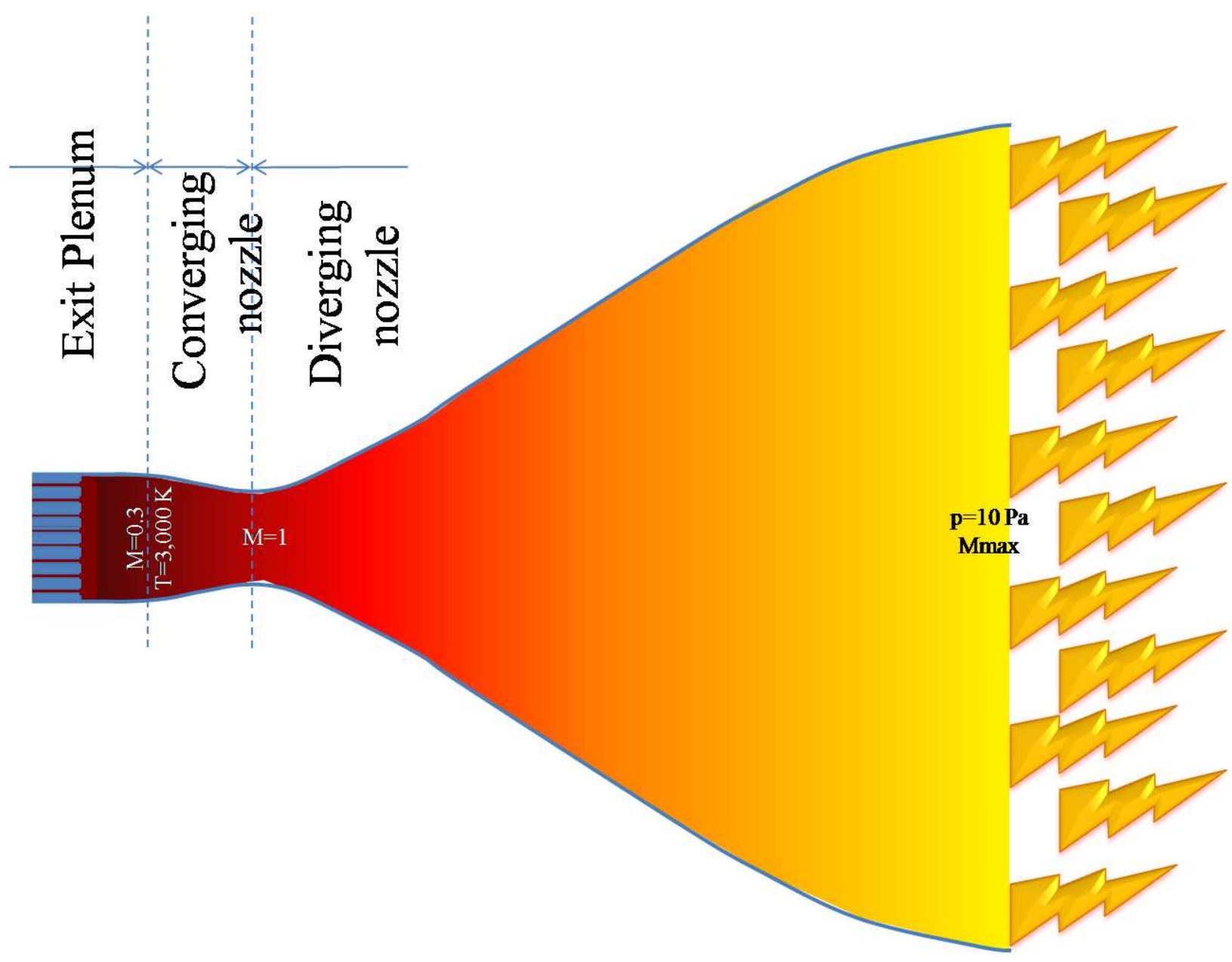

Figure 7-1: Thrust chamber and its principal characteristics 


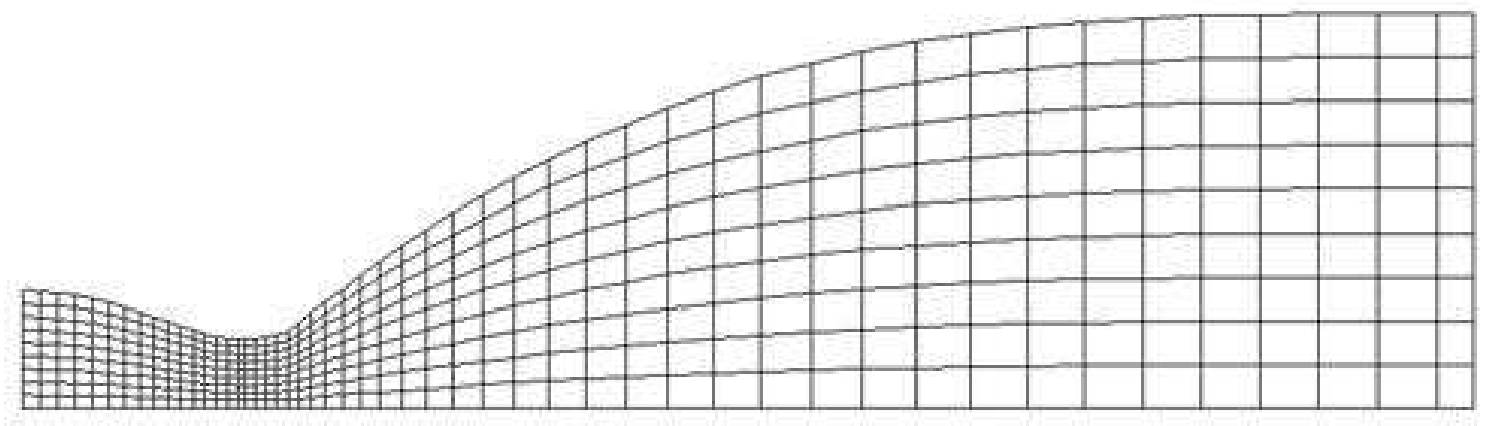

Figure 7-2: Coarser grid

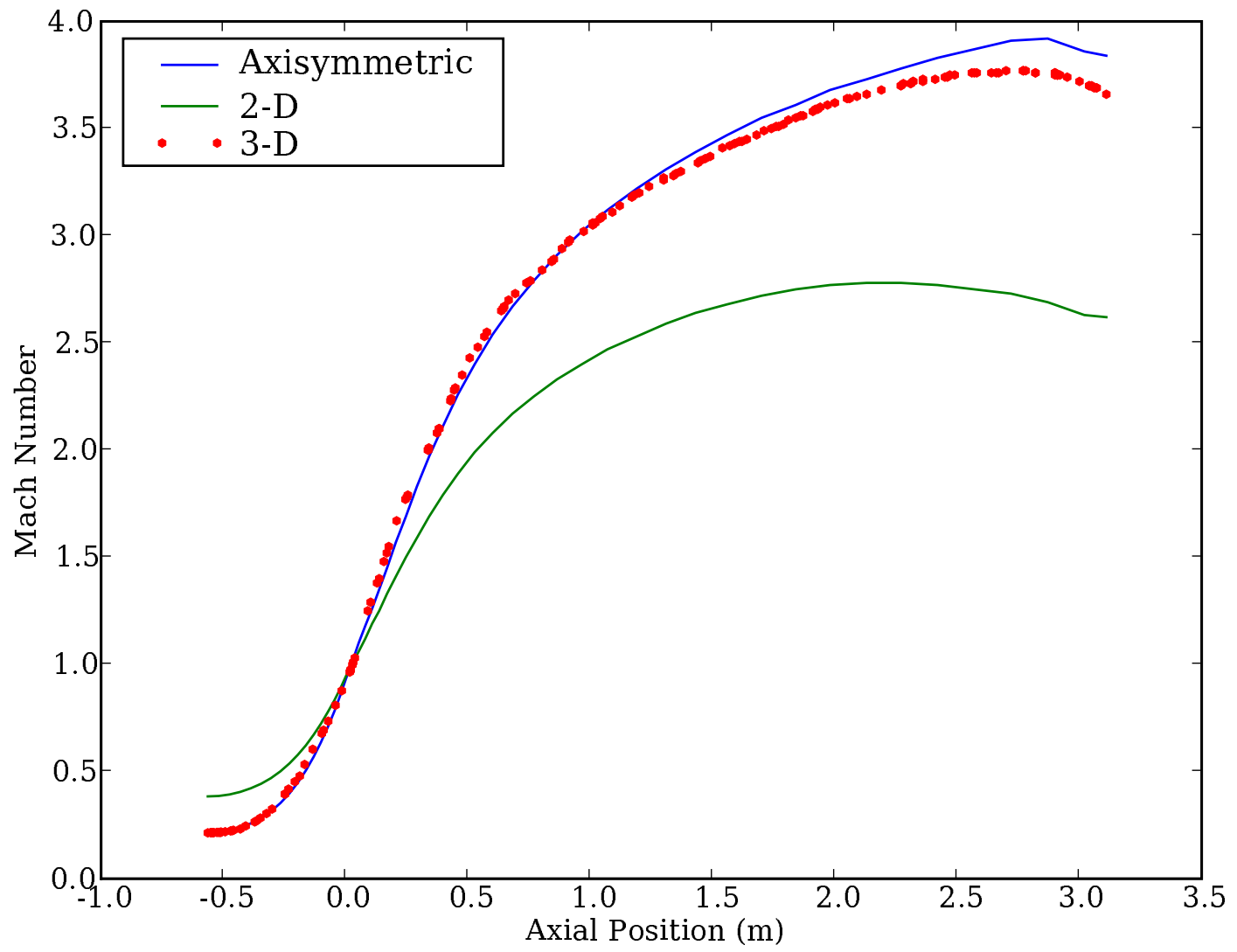

Figure 7-3: Comparison of the Mach number profile for the axisymmetric and 2-D models 
Table 7-3: Test cases for different design parameters

\begin{tabular}{llllllll}
\hline Case name & $R c$ & $\theta_{a}$ & $A_{\text {exit }}$ & $\theta$ & $L$ & $v_{\max }$ & note \\
\hline Grid1 & 2 & 20 & 1 & 20 & 0.7 & & $\mathrm{x}$ \\
Grid2 & 2 & 40 & 1 & 20 & 1 & 12724 & $\mathrm{o}$ \\
Grid3 & 3 & 20 & 1 & 20 & 1 & 12713 & $\mathrm{o}$ \\
Grid4 & 2 & 40 & 1 & 20 & 2 & 12621 & \\
Grid5 & 3 & 20 & 1 & 20 & 2 & 12598 & \\
Grid6 & 3 & 40 & 1 & 20 & 2 & 12594 & \\
Grid7 & 2 & 30 & 1.5 & 20 & 3 & 13513 & \\
Grid8 & 3 & 30 & 1.5 & 20 & 3 & 13509 & \\
Grid9 & 4 & 30 & 1.5 & 20 & 3 & 13510 & \\
Grid16 & 1 & 30 & 1.5 & 20 & 3 & 13550 & \\
Grid17 & 1.5 & 30 & 1.5 & 20 & 3 & 13517 & \\
Grid18 & 0.5 & 30 & 1.5 & 20 & 3 & & $\mathrm{x}$ \\
Grid19 & 0.8 & 30 & 1.5 & 20 & 3 & & $\mathrm{x}$ \\
Grid20 & 1.25 & 30 & 1.5 & 20 & 3 & 13522 & \\
Grid21 & 0.99 & 30 & 1.5 & 20 & 3 & 13535 & \\
Grid22 & 1.1 & 30 & 1.5 & 20 & 3 & 13526 & \\
Grid23 & 1 & 30 & 1.5 & 10 & 3 & 13544 & \\
Grid24 & 1 & 30 & 1.5 & 15 & 3 & 13567 & \\
Grid25 & 1 & 30 & 1.5 & 25 & 3 & 13540 & \\
Grid26 & 1 & 30 & 1.5 & 30 & 3 & 13619 & \\
Grid27 & 1 & 30 & 1.5 & 40 & 3 & 14353 & \\
Grid28 & 1 & 30 & 1.5 & 50 & 3 & 14648 & $\mathrm{o}$ \\
Grid29 & 1 & 30 & 1.5 & 60 & 3 & 14701 & $\mathrm{o}$ \\
Grid30 & 1 & 30 & 1.5 & 55 & 3 & 14710 & $\mathrm{o}$ \\
Grid37 & 1 & 30 & 1.5 & 40 & 4 & 14333 & \\
Grid38 & 1 & 30 & 1.5 & 40 & 2 & 13732 & \\
Grid39 & 1 & 30 & 1.5 & 40 & 3.5 & 14351 & \\
Grid40 & 1 & 30 & 1.5 & 40 & 2.5 & 14172 & \\
Grid41 & 1 & 30 & 1.5 & 40 & 3.2 & 14355 & \\
Grid42 & 1 & 30 & 1.5 & 40 & 3.3 & 14367 & \\
Grid43 & 1 & 30 & 1.5 & 40 & 3.4 & 14342 & \\
Grid44 & 1 & 25 & 1.5 & 40 & 3.3 & & $\mathrm{x}$ \\
Grid45 & 1 & 35 & 1.5 & 40 & 3.3 & $\mathbf{1 4 3 5 8}$ & \\
Grid46 & 1 & 45 & 1.5 & 40 & 3.3 & 14340 & \\
\hline & & & & & & &
\end{tabular}




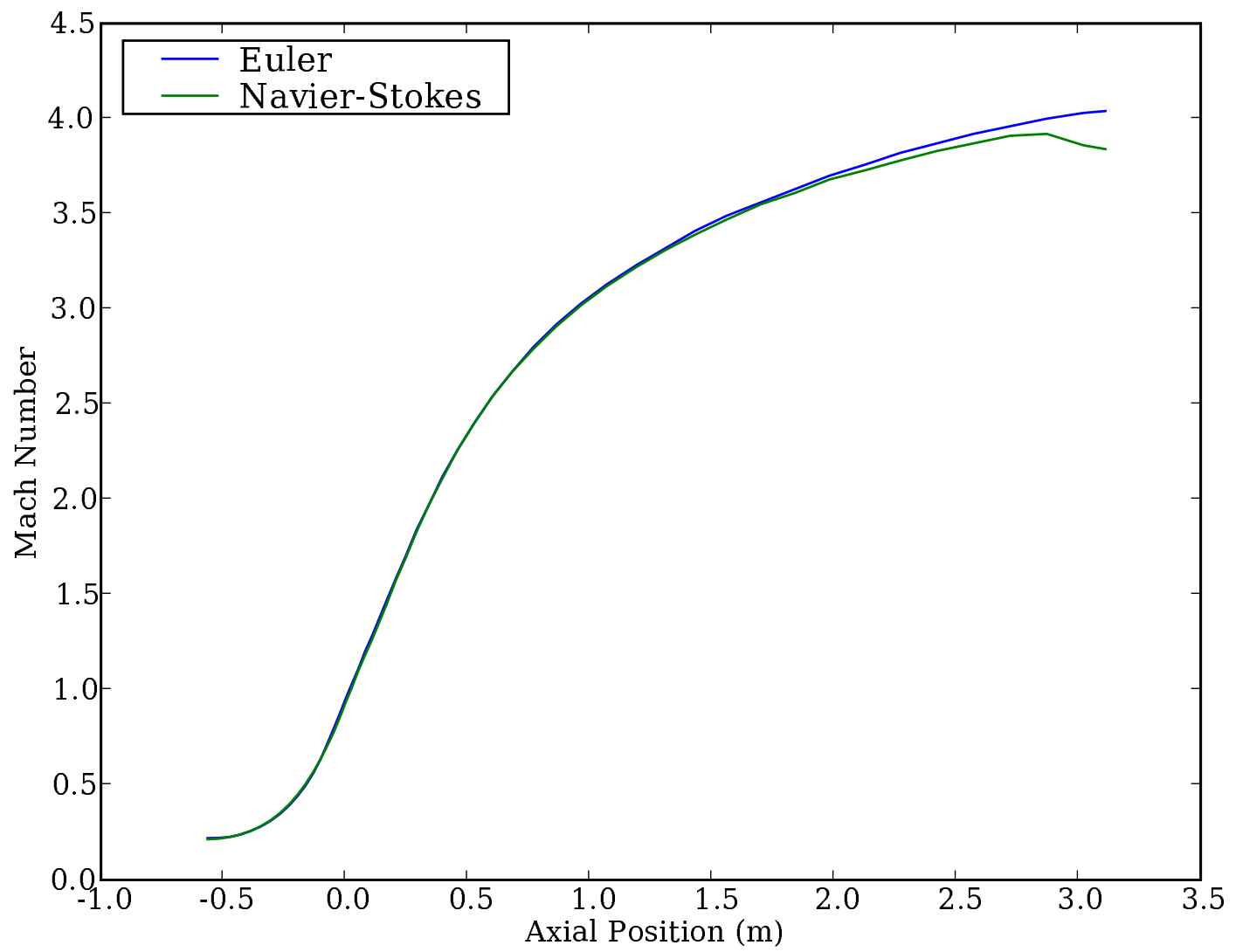

Figure 7-4: Mach number profiles for an inviscid and viscous flow of hydrogen 


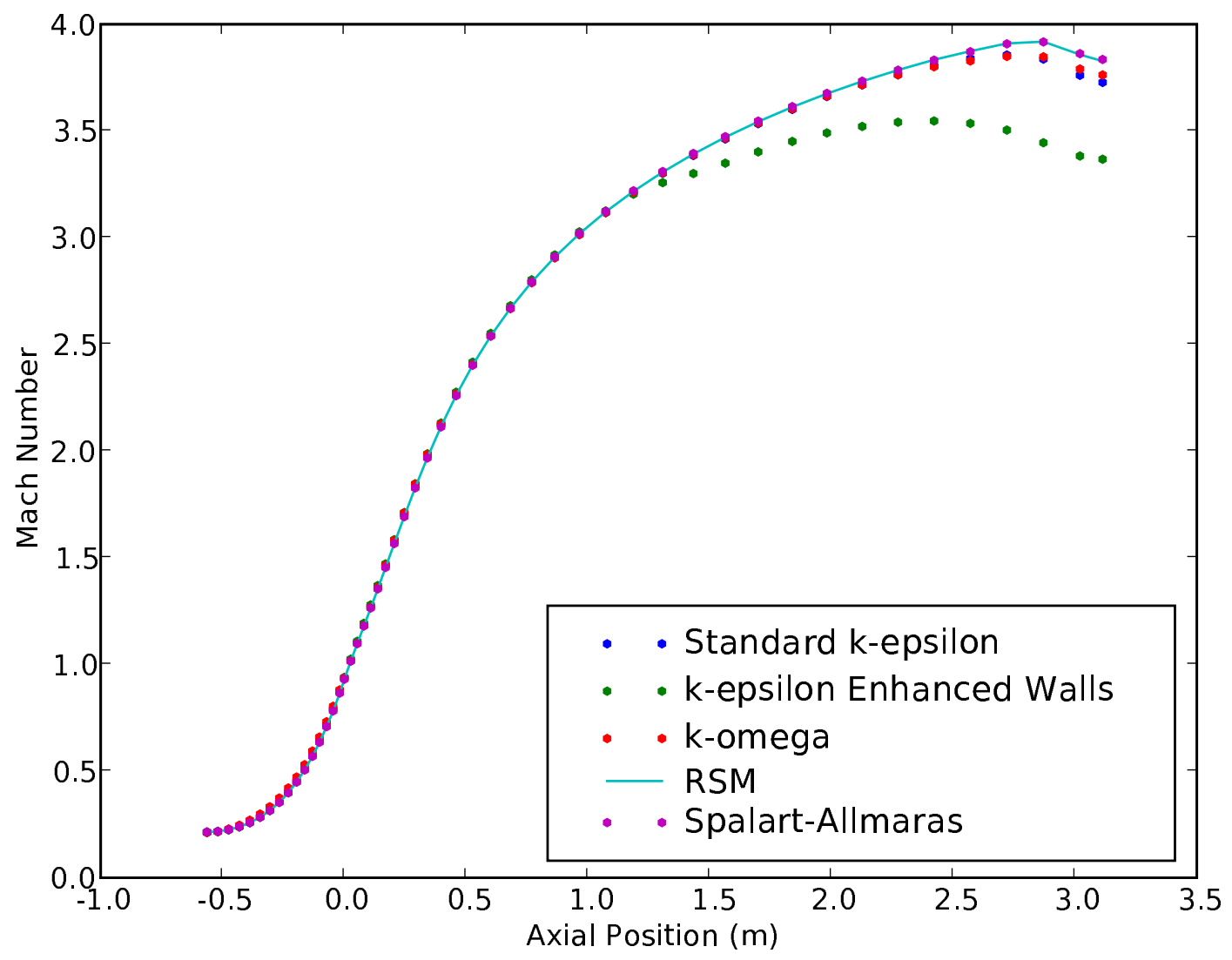

Figure 7-5: Mach number profiles obtained for different turbulence models 


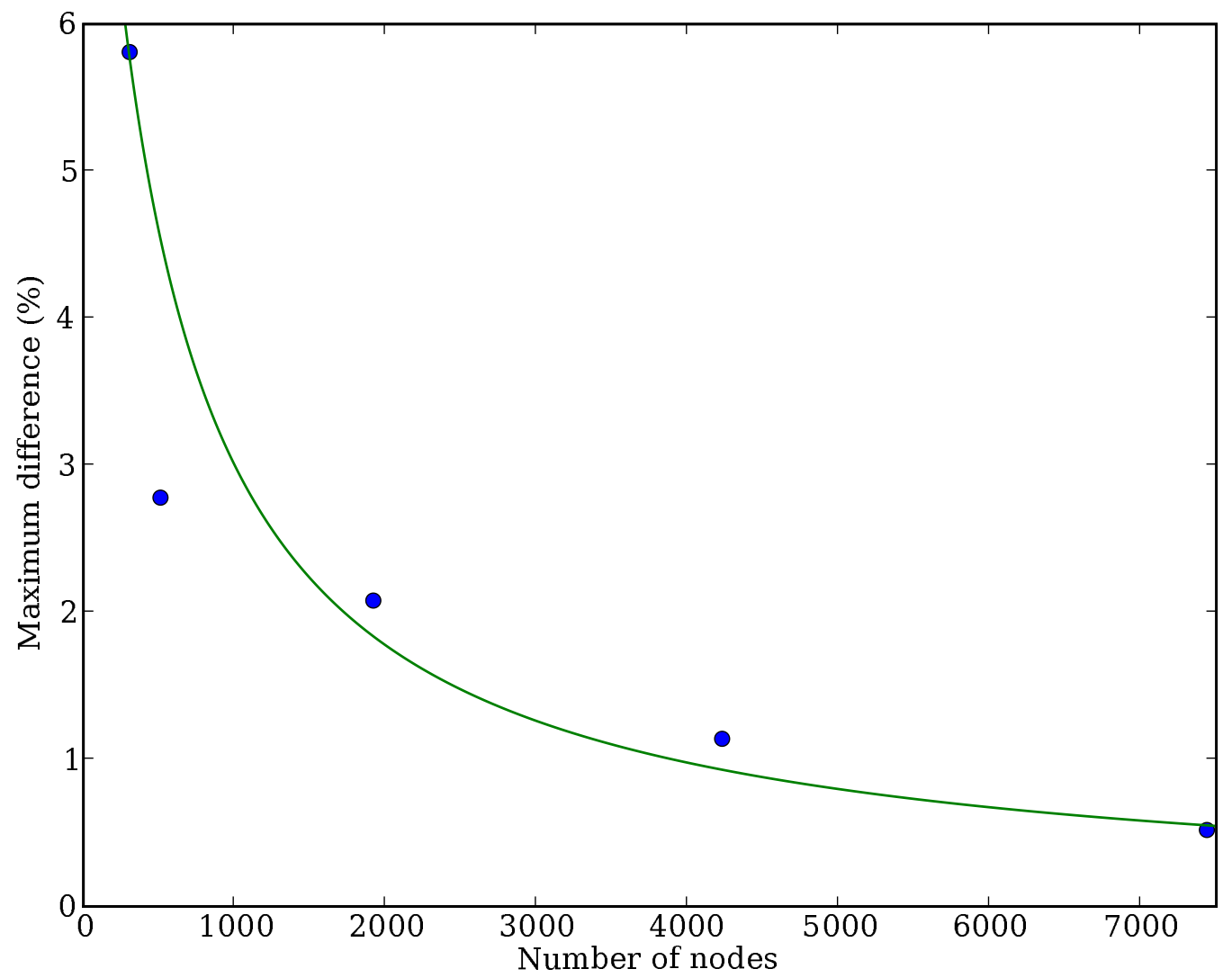

Figure 7-6: Relative difference in the maximum Mach number between a given grid and the finest grid 

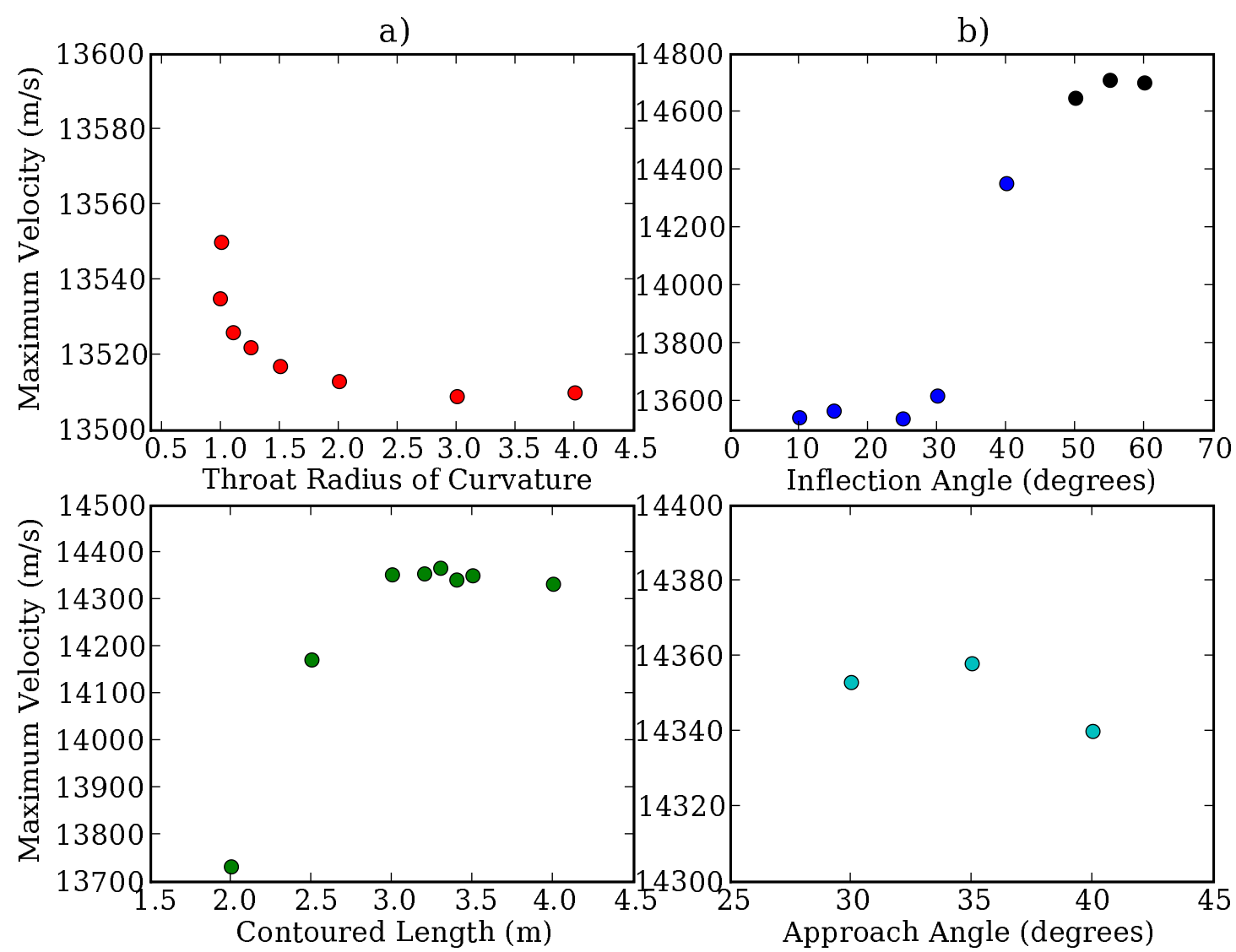

Figure 7-7: Influence of the design parameters on the maximum velocity on the exhaust plane - a) Influence of the throat ratio of curvature $(R c) \theta_{a}=30^{\circ}: \theta=20^{\circ}$ and $L=3$ $\mathrm{m}-\mathrm{b})$ Influence of the inflection angle $(\theta)$ : the black dots corresponds to designs where the exhaust velocity is not uniform $R c=1, \theta=30^{\circ}$ and $L=3 \mathrm{~m}-\mathrm{c}$ ) Influence of the contoured length $(L): R c=1, \theta_{a}=30^{\circ}$ and $\theta=40^{\circ} \mathrm{d}$ ) Influence of the approach angle $\left(\theta_{a}\right): R c=1, \theta=40^{\circ}$ and $L=3.3 \mathrm{~m}$ 


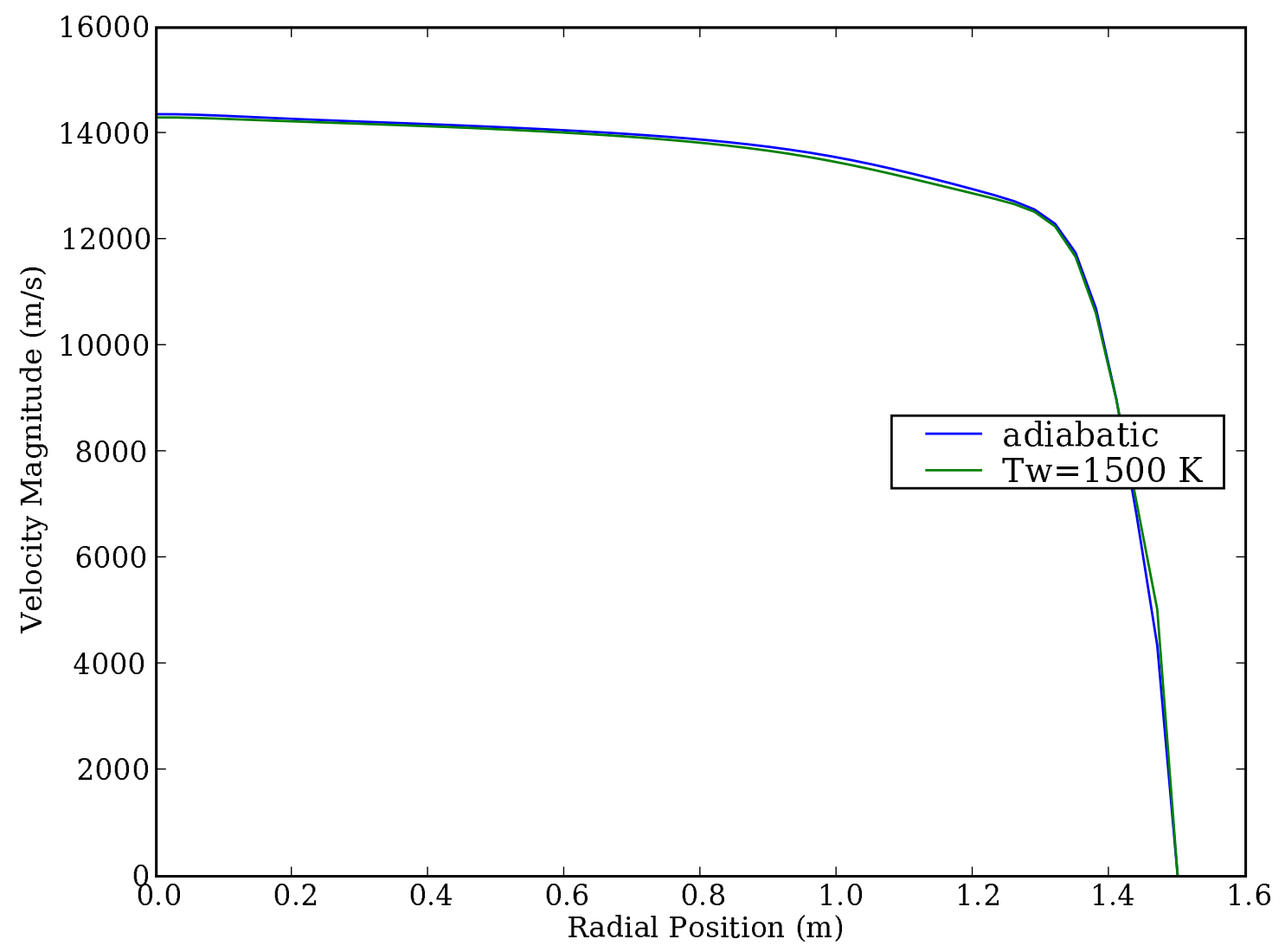

Figure 7-8: Velocity magnitude profile on the exhaust plane of the thrust chamber 


\section{CHAPTER 8 \\ COUPLING OF COARSE AND FINE MESH SOLVERS}

\subsection{NuRok: a 1-D System Code for Simulation of NTP Thermal-Hydraulics}

NuRok goal is to simulate complete nuclear thermal engine systems in order to compare performances of several types of engines. The code was developed in 1993 [5]-[78] and Plancher [101] brought additions in 2002. Since the nineties, there has been a renewed interest in thermal nuclear propulsion. It came after a long research hiatus after the NERVA program cancelation in 1972. The research in this area therefore has been focusing on regaining the knowledge gained in the NERVA program. It resulted in a large variety of core and engine system designs. Most of them are a variation of the NERVA design: prismatic fuel elements and full topping expander cycle. Therefore, he NuRok system code aims at simulating the different designs under development and to assess how the different components of the system are affected by each other and by the operating conditions.

The NuRok program is composed of several FORTRAN subroutines, each simulates a component of the NTP system. The program accounts for the real properties of hydrogen. Typically, hydrogen is the working fluid, coolant and propellant at the same time. The code models the designs described below and sketched Figure 8-1. The schematics does not show the actual piping network but shows the connections between the propulsion components and how the flow is regulated.

- Rocketdynes' nuclear vapor thermal rocket: This expander engine cycle is called a vapor rocket because the uranium fuel is in the form of uranium tetrafluoride at high temperature. The gas is embedded in fuel channels.

- NERVA derivative: It consists of the NERVA design adapted to today's technology. Typically it is a dual turbo-pump topping cycle with direct cooling. The main particularity of this design is the use of structural tie-tubes inside the nuclear core.

- Pratt and Whitney XNR2000: This system is meant to work at much lower thrust levels $(25,000 \mathrm{lbf}$ instead of 75,000 lbf for NERVA derivative and the nuclear vapor thermal rocket). The core uses a neutron fast spectrum and a two-pass coolant configuration is employed. The gas goes through a pre-heater core on the outer 
diameter of the nuclear core and then though the central core without being mixed. This design enables flexibility and lower fuel peak temperatures. Two types of XNR2000 systems are considered. They differ by the system connections as seen Figure 8-1.

- Square lattice honeycomb (SLHC): In the three previous designs, the fuel is an hexagonal matrix of fissile materials pierced with thousands of holes to enable the hydrogen flow, which removes the heat from fissions. Manufacturing of such fuel is very challenging. A new type of fuel, called the Square Lattice Honeycomb, facilitates the manufacturing steps: the fuel originally under the form of a powder is compacted into 1 to $2 \mathrm{~mm}$ thick grooved wafers which then are interconnected to form a "honey puck" type fuel subassembly. The subassemblies are inserted into a graphite matrix to form the core of the propulsion. The flow map is the same as for the Nuclear Vapor Thermal Rocket, except in the core where the cross-sectional area of a coolant channel is a square.

All of these designs are full-topping expander cycles. The term 'full-topping' is used because all of the propellant is exhausted through the nozzle at the core exit temperature. It differs from the 'hot-bleed' cycle where the coolant from the thrust chamber and pump outlet are mixed and used to drive the turbine and which sends the cooler turbine exhaust through a separate nozzle. Originally, the NERVA program considered the hot bleed configuration for its simplicity. The newer designs favor the full topping expander configuration because performs better.

The NuRok core returns information about the entire engine system such as mass flow rate, pressure at any location, maximum fuel temperature, for example, once a set of operating conditions is converged upon. The operating conditions are defined by the rocket thrust and the thrust chamber inlet pressure and temperature.

Each of the components of the engine are described by an independent subroutine: for example, turbine and pump are each described by a subroutine. The core is represented by its hot channel. The core subroutine calls for other subroutines such as the ones for the pressure drop in a tube, the heat flux profile, the clad temperature, the friction factor... The subroutines work as modules and the different design configurations are described by simply linking the different subroutine/modules accordingly. 


\subsubsection{Description of NuRok Core Subroutine and One-Dimensional Correla- tions}

The pressure drop across a nuclear rocket core is of major importance since it significantly influences the other engine components. Its analysis should be as accurate as possible. However, it is not an easy task because of the complex nature of the core flow. The ultimate goal of the core subroutines in the system architecture of NuRok is to adjust the core power until a desired exit temperature is reached. Then, the code derives the resulting pressure drop across the system. The thermodynamic properties of the propellant are known at the inlet of the core. The core subroutines must analyze the flow transition from the inlet chamber to the coolant channels, flow through these coolant channels, and the transition from the channels to the thrust chamber. The sudden contraction from the inlet chamber into the coolant channels, and the expansion that occurs as the hydrogen coolant exits these channels are the plenum effects. The contraction and expansion of the flow is considered instantaneous and thus adiabatic. A sharp pressure drop is considered at the inlet and exit plenums. In the 1D model developed, the pressure drop is a function of the area difference between the core inlet or outlet chambers and the coolant sub-channels. The pressure drop is modeled in the upper and lower plenum by:

$$
\Delta p=\frac{1}{2} k_{c} \rho v_{2}^{2}
$$

for an area contraction and

$$
\Delta p=\frac{1}{2} k_{c} \rho\left(v_{1}-v_{2}\right)^{2}
$$

for an area expansion, where $k_{c}$ is the contraction loss coefficient defined by:

$$
k_{c}=\left[1+\frac{A_{1}+A_{2}}{A_{1}-A_{2}}\right]^{-1}
$$

Since all of the coolant channels are geometrically identical and mean radial conditions are used in the analysis, only one channel must be analyzed to obtain an axial flow profile though the core. This axial flow profile includes information about the propellant 
temperature, pressure, velocity, Mach number, and thermodynamic properties. The profile is generated by discretizing a one-dimensional axial representation of the square channel into 50 elements. The conditions derived at the previous element's exit node are set equal to the current element's inlet node and used to calculate the exit node conditions of this current element. The desired inlet thrust chamber temperature is input by the user and equals the core exit temperature. It dictates the core power and the mass flow rate at a given thrust level. To calculate the propellant temperature rise, the system is considered under steady-state conditions. A linear heat generation rate (LHGR) is derived from the heat flux profile at each node element. The enthalpy rise across a coolant channel element can be calculated from:

$$
\Delta h=\frac{\operatorname{LHGR} \Delta z}{\dot{m}}
$$

The inlet node pressure is used in conjunction with the exit enthalpy to find the exit temperature and hence the bulk temperature profile is derived. The temperature rise in the core is of the order of $2,500 \mathrm{~K}$ so hydrogen properties across the core cannot be considered constant. Also because each coolant channel is very small, Mach number of the flow can be greater than 0.3 and the compressible nature of the flow has to be accounted for. Finally, heat exchanges in the boundary layer of the channel must be considered. The one-dimensional correlation accounts for boundary layer heat transfer and compressibility of the fluid. The equation:

$$
\Delta p=\dot{m}^{2}\left(\frac{1}{\rho_{1}}-\frac{1}{\rho_{2}}+2 f_{f} \frac{\Delta z}{D}\left(\frac{1}{\rho_{m}}\right)\right)
$$

agrees best with experiment and computer simulations and is usually recommended (Kays and Perkins, 1985). It is the equation used for pressure drop calculations in NuRok. The value $\frac{1}{\rho_{m}}$ is the integrated mean value across the duct section ad can be approximated by:

$$
\frac{1}{\rho_{m}} \simeq \frac{1}{2}\left(\frac{1}{\rho_{1}}+\frac{1}{\rho_{2}}\right)
$$


where $f_{f}$ is the fanning friction factor. Different correlations can be found in the literature. The most commons are summarized in Table 8-1. The definition implemented in NuRok is the correlation by Koo.

Finally the fuel surface temperature profile must be computed: once the surface temperature is known it is possible to derive the maximum fuel temperature by considering conductive heat transfer in the fuel. It is important to compute the maximum fuel temperature since it limits the core operating power: for a given operating condition one has to make sure that the maximum fuel temperature is low enough compared to the fuel melting point. To compute the surface temperature at any axial location of the core we need the information on bulk temperature and heat transfer coefficient:

$$
T_{w}=T_{b}+\frac{q^{\prime \prime}}{h}
$$

where $h$ is the heat transfer coefficient defined by

$$
h=\frac{N u \cdot k}{D}
$$

Colburn, Dittus-Boelter, Seider-Tate and many others developed correlations to find the Nusselt number. The most common correlations are given in Table 8-2. The relation used in NuRok is:

$$
N u=N u_{i s o}\left(\frac{T_{w}}{T_{b}}\right)^{n}
$$

where $n=-\left[\log \left(\frac{T_{w}}{T_{b}}\right)\right]^{\frac{1}{4}}+0.3$ (Kays and Perkins, 1985) and the isotropic Nusselt number is derived with the Petukov (1970) relation and an axial distance correction. This correction is needed since there are entry effects due to the thin thermal boundary layer at the inlet of the channel. A correction function by Pierce is implemented, and little further research will be done on the axial correction factor since for the channel of concern $x / D \sim 750$. The Pierce correction is:

$$
N u(z)=N u\left[1+0.3\left(\frac{z}{D}\right)^{-0.7}\right]
$$


To illustrate the shortcomings of the one-dimensional correlations, we compare relation 8-1 to a three-dimensional CFD simulation of a channel with sudden area loss. The model represents a circular tank which area reduces to a square channel as seen Figure 8-2. The radius if the tank is $5 \mathrm{~mm}$ and the edge of the square channel is equal to $1 \mathrm{~mm}$. The simulation is done using the $\mathrm{k}-\epsilon$ segregated solver of a tetrahedral mesh with 118,042 computational cells. The test section is $5 \mathrm{~cm}$ long and the walls are considered adiabatic. The operating conditions are typical of the hydrogen conditions at the inlet of the SLHC core: 1 atm and 186 K. Figure 8-3 shows the static pressure evolution along the axis of the test-section. The inlet of the test section is located at $z=-2 \mathrm{~cm}$ and the area contraction at $z=0$. The left-hand portion corresponds to the pressure drop over the tank. It is almost negligible. The middle portion corresponds to the abrupt pressure drop due to the flow contraction and the right-hand portion to the pressure drop over the channel. For comparison with the analytical formula 8-1, we only consider the abrupt pressure drop. Results for diverse inlet velocities is given Table 8-3. Overall, the correlation underestimates the importance of the pressure drop at the area contraction. As the inlet velocity increases, the error reduces, however, the difference is still very consequent $(272 \%)$.

This simple example illustrates how the 1-D correlations may fail to predict the flow behavior accurately. This is why this Chapter will present further down the coupling technique used to increase NuRok accuracy. Beforehand, let us review the upgrades we brought to the system code itself.

\subsubsection{NuRok Upgrading}

The first version of NuRok was developed in 1993 and was becoming obsolete. We made several adjustments to update the old version. 


\subsubsection{Update to FORTRAN 90}

First, we updated the program to FORTRAN 90 to bring more stability to the solver (obsolescence of the COMMON procedure for example) and help compatibility with the fine mesh subroutines.

\subsubsection{Levels of thrust}

We also modified the code such that it could handle larger thrust values and thus larger core powers. The initial code would consider the diameter of the core to be constant and equal to the design diameter. The NuRok versions by Given and Plancher would enable runs for thrusts ranging between 10,000 and 70,000 lbs. If the thrust levels were greater than 70,000 lbs, the solver would diverge and the Mach number in the core coolant channels would increase to critical values. Critical Mach number values in nuclear systems corresponds to flows which are close to being transonic. If the Mach number in the core is too high, we can imagine that with fuel expansion and swallowing the flow area is reduced, leading to a local increase in Mach number. The flow reduction might even accelerate the flow such that it becomes supersonic and generates shocks in the core of the propulsion. Such a flow behavior is very dangerous for the core stability and to be prohibited. Therefore, greater levels of thrusts for the Nuclear Thermal Propulsion require greater flow area in the core. This means larger core diameters with the same density of coolant channel. Increasing the core diameter increases the number of coolant channels and then increases the flow area available for the coolant, reducing the local velocity. This technique avoids critical Mach numbers of the flow in the core. The critical Mach number is set to 0.4. This value provides sufficient safety margin in the core.

Practically, the flow Mach number is computed in the core with its initial design diameter. Then the flow is iterated such that, as long as the Mach number is greater than 0.4 (critical Mach number) the core diameter is increased by 5\%. Table 8-4 presents the different characteristics of the SLHC systems for diverse thrust levels. The thrust chamber inlet temperature and pressure are 2,800 $\mathrm{K}$ and 500 psi, respectively. 


\subsubsection{Foam fuel}

Researchers and Engineers at NASA's George C Marshall Space Flight Center and INSPI are interested in tri-carbide, open-cell foam fuel matrix for nuclear propulsion. Usually, density of the foam fuel is of the order of $20 \%$. The core subroutine is modified to allow modeling of such fuel. The user can model a NTP system with foam fuel by selecting the flow area ratio, corresponding to the foam density. The code works for densities between 17 and 50\%. Table 8-5 presents the system characteristics for diverse foam fuel densities. This is only an approximation since very few research has been done on pressure drop and heat transfer. The foam fuel still need to be qualified on a materials standpoint first, before leading further research. However, the simple study presented here shows that the only property the variation in density influences is the pressure drop across the core. The cases of comparison exhibit a level of thrust equal to 50,000 lbs and nozzle inlet temperature and pressure equal 2,800 K and $500 \mathrm{psi}$, respectively. The power transferred to the fluid is equal to $1024 \mathrm{MW}$ for all densities and the maximum cladding temperature in the core is $2,807 \pm 1 \mathrm{~K}$. The pressure drop across the core increases as the foam density (and fluid flow area) decreases.

\subsubsection{Hydrogen dissociation}

Older versions of NuRok account for the real properties, except for dissociation. Since we are interested in performances of the NTP associated to low-pressure thrust chamber (Chapter 7), it becomes important to model properly hydrogen molecular weight. The implementation is the same as for the fine mesh solvers (Section 4.1.2).

\subsubsection{Carbon dioxide}

Some nuclear propulsion designs in the early stages of development considered substituting Hydrogen with Carbon Dioxide. To investigate the qualities of carbon dioxide as a working fluid in nuclear thermal propulsion NuRok is modified: $\mathrm{CO}_{2}$ properties are given in Chapter 4 and are implemented in the programs. The user is now asked to choose 
a coolant to perform his calculations. The user can choose between helium, hydrogen and carbon dioxide.

\subsubsection{Recommendations for NuRok Users}

When running NuRok for a set of operating conditions, namely exhaust thrust (Th (lbf)), thrust chamber pressure $\left(P_{T C}(\mathrm{psi})\right)$ and thrust chamber temperature $\left(T_{T C}(\mathrm{~K})\right)$, the solver asks for user guesses on the mass flow rate in the system $(\dot{m}(\mathrm{~kg} / \mathrm{s}))$, the pump outlet pressure $\left(P_{\text {out }}(\mathrm{psi})\right)$ and the core power $(Q(\mathrm{MW}))$. If the guess is not appropriate, the solver will not converge. Therefore, we recommend that the user follows the hints below. It guarantees NuRok convergence. Quantitative values are given as an example for the SLHC system under the following conditions (which corresponds to an ISP of $\left.950 \mathrm{~s}^{-1}\right)$ :

$$
\delta=1 \mathrm{~mm} ; T_{T C}=2,800 \mathrm{~K} ; P_{T C}=500 \mathrm{psi} ; T h=50,000 \mathrm{lbf}
$$

where, $\delta$ is the wafer thickness.

\subsubsection{Mass Flow Rate}

A low value of the mass flow rate helps the convergence. In this case $\dot{m}=24.02(\mathrm{~kg} / \mathrm{s})$. The convergence is reached for guesses up to $30 \mathrm{~kg} / \mathrm{s}$. By default, we would recommend an initial guess of $15 \mathrm{~kg} / \mathrm{s}$.

\subsubsection{Pump Outlet Pressure}

The solver is very sensitive to this value. It is recommended to input a pressure close but no greater than twice the thrust chamber inlet pressure. In our example, the pump outlet pressure we derive is equal to 1048 psi. However, the code only converges if the initial guess is within 700 and 1000 psi. Note that in the newer versions of NuRok the pressure is automatically guessed by the code.

\subsubsection{Core Power}

An overestimated guess of the core power helps the convergence. In our example, $Q=$ 1041.6 MW. The solver converges if the initial guess is within 1,000 and 2,000 MW. 


\subsection{Themis: A Coupled Coarse-Fine Mesh Simulation Tool for Simulation of NTP Thermal-Hydraulics}

NuRok is composed of several subroutines. We can identify areas of the code where the one dimensional simulation lacks accuracy. On the schematics of a full-toping, by-passed NTP system Figure 8-4 we identified several portions of the system which would benefit from a mesh and solver refinement. They are the inlet and exit plenum, mixing junctions, coolant channels and thrust chamber. Chapter 7 detailed the reasons for a 2-D analysis of the thrust chamber. In Chapter 6 we saw the case of the coolant channel in the VHTR. Since the NTP is also a nuclear system with gas flow, the remarks we made also apply to the NTP coolant channels.

At the inlet and exit of the core there is a sudden restriction and expansion of the flow area, respectively. The particular geometry induces turbulent effects as well as anisotropy of the flow. The nodal code of NuRok cannot account correctly for these multi-dimensional phenomena. A detailed CFD analysis would be required to get an estimation of the importance of the flow anisotropy in the exit plenum, for example. Besides, hydrogen is not heated uniformly in all the core channels. Because the thermal flux generated by neutron fission is not isentropic over the core, certain channels will be hotter: the gas enters the exit plenum with different temperatures. The temperature gap could be over dozens of degrees. Typically, a compact nuclear core is composed of 24,000 coolant channels. Representation of even $1 / 6$ th of the core would require several million of computational cells. This is two computationally expensive for our feasibility study here. However, future work could be led in this direction.

The coolant channels and thrust chamber properties have been detailed in Chapters 6 and 7.

\subsubsection{Treatment of the Interfaces}

There are two types of interfaces, the interface from the system code to the fine mesh solver and the interface from the fine mesh solver to the system code. Therefore, we need 
to treat the interface from 1-D to 2-D axisymmetric and vice versa. Figure 8-5 shows the coupling schematics of a fine mesh solver for the hot channel of the core to the rest of the NTP system code.

\subsubsection{One-dimension to two-dimension axisymmetric}

$$
\text { (system code) } \Rightarrow(\text { CFD solver })
$$

This type of interface is used to input the boundary conditions extracted from the system code calculations into the CFD solver. It is the case of the inlet velocity components, inlet density and exit pressure. Since the system code return only one values of each parameter at the boundaries of the system of consideration, we will implement uniform boundary condition values in the CFD solver. This is a trivial but accurate way to treat the interface $1-\mathrm{D}$ to $2-\mathrm{D}$ axisymmetric

\subsubsection{Two-dimension axisymmetric to one-dimension}

$$
(\mathrm{CFD} \text { solver }) \Rightarrow(\text { system code })
$$

This type of interface is used to extrapolate the values computed by the fine mesh solver and input them back into the system code. This type of interface is needed to implement the pressure at the inlet of the system, the velocity and temperature at the exit of the system.

Typically, the pressure profile in a heated tube is constant on the radial profile. Therefore, the pressure that we compute on the inlet plane of the hot channel can be averages and the averaged pressure input in the coarse mesh solver for a realistic representation of the pressure profiles. At the exit of the hot channel, we need to interpolate the velocity components and temperature. Usually, for a turbulent flow, the radial velocity profile is almost flat. Thus, we obtain a single value of the velocity magnitude at the exit of the hot channel by extracting the maximum value derived on the exit plane and inputting it in the coarse mesh. 
Input of the temperature is a little bit more challenging: As seen on Figure 8-6, one computational node in the axisymmetric model corresponds to an annulus area on the exit plane of the channel. Therefore, to get an idea of the average temperature at the exit of the channel, we weight the temperature derived at each computational node by its associated area. The temperature which is input in the coarse mesh therefore is:

$$
T_{\text {exit }}=\frac{\sum_{i=1}^{N} T_{i} \pi\left(r_{i+1}-r_{i}\right)^{2}}{A_{\text {exit }}}
$$

where $A_{\text {exit }}$ is the cross-sectional area of the channel, $N$ the number of computational nodes, $T_{i}$ the temperature derived at node $i$ and $r_{i}$ the interface between node $i-1$ and node $i$ radius.

\subsubsection{Themis Results in the Case of NERVA Derivative Systems}

To study the feasibility of coupling coarse and fine mesh solvers, we study the NERVA derivative system with the following characteristics:

$$
\left\{\begin{array}{l}
100,000 \text { lbf of thrust } \\
\text { thrust chamber temperature: } 3,000 \mathrm{~K} \\
\text { thrust chamber temperature: } 1,000 \mathrm{psi}=6.89 \mathrm{MPa}
\end{array}\right.
$$

The overall system is modeled with the coarse mesh solver and the coolant channel with the fine mesh solver. Modeling of the channel is very similar to the model we presented in Chapter 6. The main differences are:

- the fluid of interest is hydrogen

- the thermal boundary condition at the wall is given by a heat flux profile

- definition of the pressure boundary condition (see below)

The dimensions of the coolant channels in the NERVA derivative system are:

$$
\left\{\begin{array}{l}
D=2.54 \mathrm{~mm} \\
L=1.27 \mathrm{~m}
\end{array}\right.
$$


The heat flux applied to the wall of the coolant channel is defined by an average heat flux weighted following the curve shown Figure 8-7. The average core heat flux is updated at every core iteration by the coarse mesh solver, such that the channel exit temperature and pressure match the design requirements specified by the user for each run. NuRok obtains the convergence on the pressure in the system by deriving the pressure drop across the coolant channel. At each iteration, the inlet pressure is considered known. On the first iteration it is interfered with the guess of the pressure drop across the pumps. The pressure is derived through the coolant channel and the exit channel is compared to the user input for the thrust chamber pressure. Depending on the difference between the two values, the pressure drop across the pumps is re-estimated and a new iteration starts. For the CFD model which is coupled to the system code, the boundary condition on pressure is located at the inlet. Traditionally, the exit pressure is known. This modification does not influence the performances of the fine mesh solver.

Table 8-6 compiles the results of the simulation. The results are compared to those obtained with NuRok only. Figure 8-8 and 8-9 show the profiles of pressure and temperature across the hot channel calculated with NuRok and Themis. The core thermal power derived with Themis is larger by $15 \%$ from the NuRok simulation. This is mainly due to the large difference in the pressure drop across the channel that is computed with the two methods. The pressure profiles are significantly different: not only the pressure prod prediction obtained with the coupled solver is three times larger than the prediction obtained with NuRok, but also the trends of the two curves seen Figure 8-8 are quite different. The temperature profiles are difficult to compare since

It is difficult to compare the temperature profiles (Figure 8-9) since the coarse model only gives one profile and the fine model gives a couple of profiles: wall temperature and temperature at the axis. The temperature profiles are very similar for the two approaches. The pressure profiles (Figure 8-8) are very different. The pressure drop 
calculated with the CFD solver is three time large than the pressure drop obtained with simpler one-dimensional correlations.

The difference between the two simulations is very sensible. It shows the importance of using the fine mesh solver. With NoRok, the overall code converges within 8 iteration, whereas with Themis, the code converges after 11 iterations. The computational time is multiplied by three. However, the overall CPU still remains under 1 min to obtain steady-state.

\subsubsection{Conclusion on the Coupled fine-coarse mesh solver}

Themis highlights the shortcomings of using a one-dimensional representation of the cooling channel. The one-dimensional representation definitively gives a very good order of magnitude of the phenomena happening in the coolant channel and the system overall. However, the calculations are more qualitative. The coupled fine-coarse mesh code with

real properties descriptions proved to be efficient and greatly increase the accuracy of the calculation while the steady-state computation remains very fast. 
Table 8-1: Fanning friction factor correlations and their range of validity [2]

\begin{tabular}{cccc}
\hline Investigator & Correlation & Range of Validity & Reynolds Range \\
\hline N.A. & $\frac{16}{R e}$ & Laminar flow & $R e<2300$ \\
Schlichting [102] & $1.33 * 10^{-3} R e^{0.52}$ & Transitional flow & $2300<R e<4000$ \\
Karmon-Nikuradse & $\frac{1}{\sqrt{f_{f}}}=4 \log \left(R e \sqrt{f_{f}}\right)-0.4$ & Turbulent flow & $2000<R e<3 * 10^{6}$ \\
Filonenko & $(1.58 \ln (R e)-3.28)^{-2}$ & Turbulent flow & $10^{4}<R e<10^{7}$ \\
Blasius & $0.079 R e^{-2.5}$ & Turbulent flow & $3000<R e<10^{5}$ \\
McAdams & $0.046 R e^{-02}$ & Turbulent flow & $6000<R e<2 * 10^{5}$ \\
Koo & $0.0014+\frac{1}{8} R e^{-0.32}$ & Turbulent flow & $2000<R e<10^{5}$ \\
\hline
\end{tabular}

Table 8-2: Nusselt number correlation for turbulent gas flow in a pipe [3]

\begin{tabular}{|c|c|c|}
\hline Investigator & Correlation & Range of Validity \\
\hline Colburn,1933 & $0.023 \operatorname{Re}^{0.8} \operatorname{Pr}^{1 / 3}$ & $\left\{\begin{array}{l}R e \geq 10^{4} \\
0.7<P r<160\end{array}\right.$ \\
\hline Dittus-Boelter,1930 & $0.023 R e^{0.8} \operatorname{Pr}^{0.4}$ & $\left\{\begin{array}{l}R e \geq 10^{4} \\
0.7 \leq \operatorname{Pr} \leq 160\end{array}\right.$ \\
\hline Seider-Tate, 1936 & $0.027 \operatorname{Re}^{0.8} \operatorname{Pr}^{1 / 3}\left(\frac{\mu_{b}}{\mu_{w}}\right)^{0.14}$ & $\begin{array}{l}R e \geq 10^{4} \\
0.7 \leq \operatorname{Pr} \leq 16,700\end{array}$ \\
\hline Notter-Sleicher, 1975 & $5.0+0.015 \operatorname{Re}^{a} \operatorname{Pr}^{b}$ where $\left\{\begin{array}{l}a=0.88-\frac{0.24}{4.0+P r} \\
b=0.33+0.5 e^{-0.6 P r}\end{array}\right.$ & $\left\{\begin{array}{l}10^{4} \leq \operatorname{Re} \leq 10^{6} \\
0.1 \leq \operatorname{Pr} \leq 10^{4}\end{array}\right.$ \\
\hline Petukov, 1970 & $\frac{\operatorname{Re} \operatorname{Pr}(f / 2)}{1.07+12.7\left(\operatorname{Pr}^{2 / 3}-1\right) \sqrt{f / 2}}$ & $\left\{\begin{array}{l}R e \geq 10^{4} \\
0.5 \leq \operatorname{Pr} \leq 200\end{array}\right.$ \\
\hline
\end{tabular}

Table 8-3: Pressure drop over a flow area contraction. Comparison between FLUENT simulation and 1-D correlation results.

\begin{tabular}{cccc}
\hline Inlet Velocity $(\mathrm{m} / \mathrm{s})$ & $\Delta P(\mathrm{~Pa}) \mathrm{CFD}$ & $\Delta P(\mathrm{~Pa})$ calc & Relative difference $(\%)$ \\
\hline 0.2 & 23.2 & 4.3 & 440 \\
0.37 & 39.5 & 8.5 & 365 \\
0.5 & 122 & 27 & 352 \\
0.75 & 261 & 30.7 & 333 \\
1 & 447 & 108 & 314 \\
1.5 & 974 & 243 & 301 \\
2 & 1687 & 571 & 195 \\
10 & 40,142 & 10794 & 272 \\
\hline
\end{tabular}



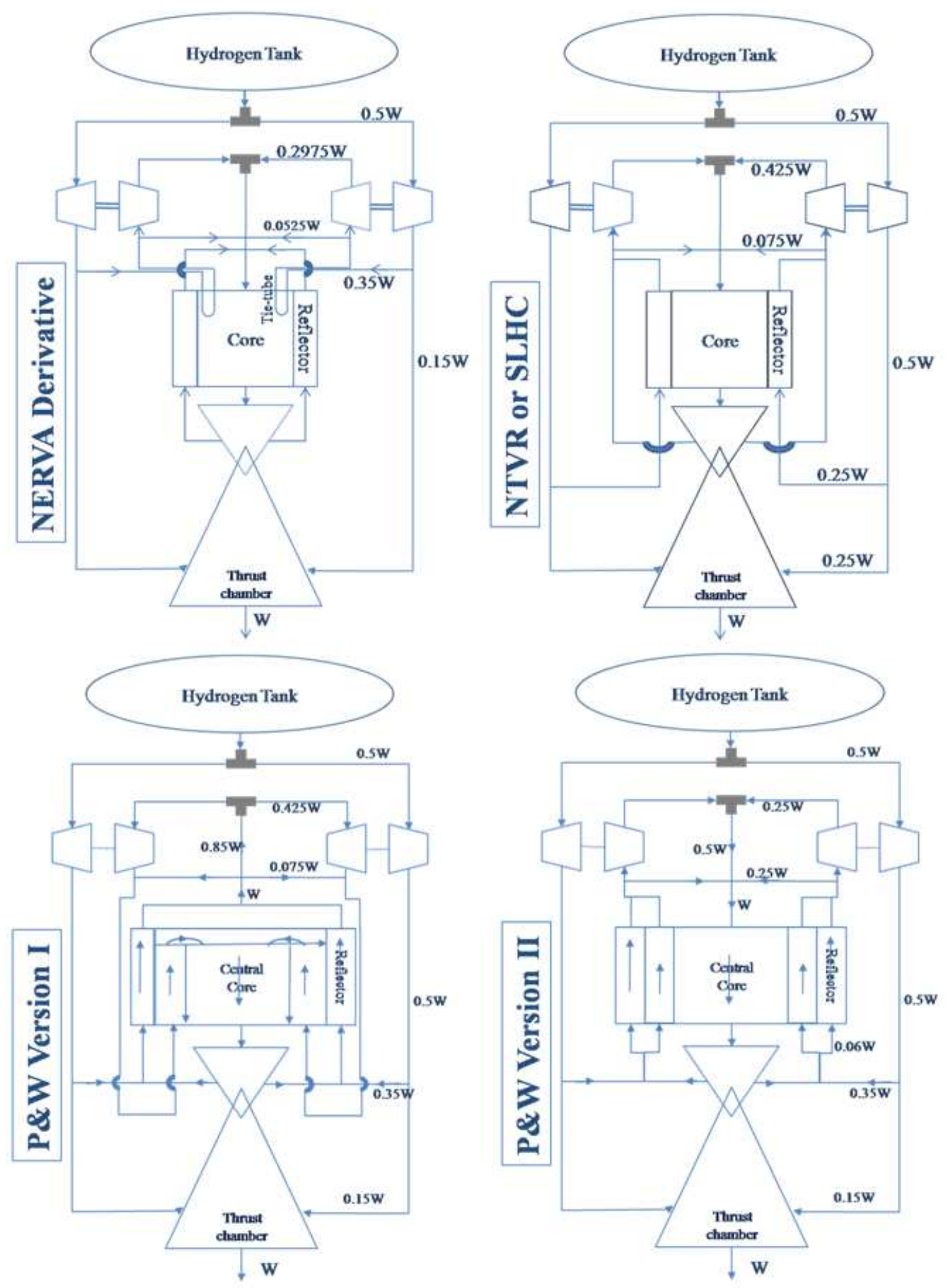

Figure 8-1: The different NTP systems modeled with NuRok: NVTR, NERVA derivative and P\&W XNR 2000 I \& II 


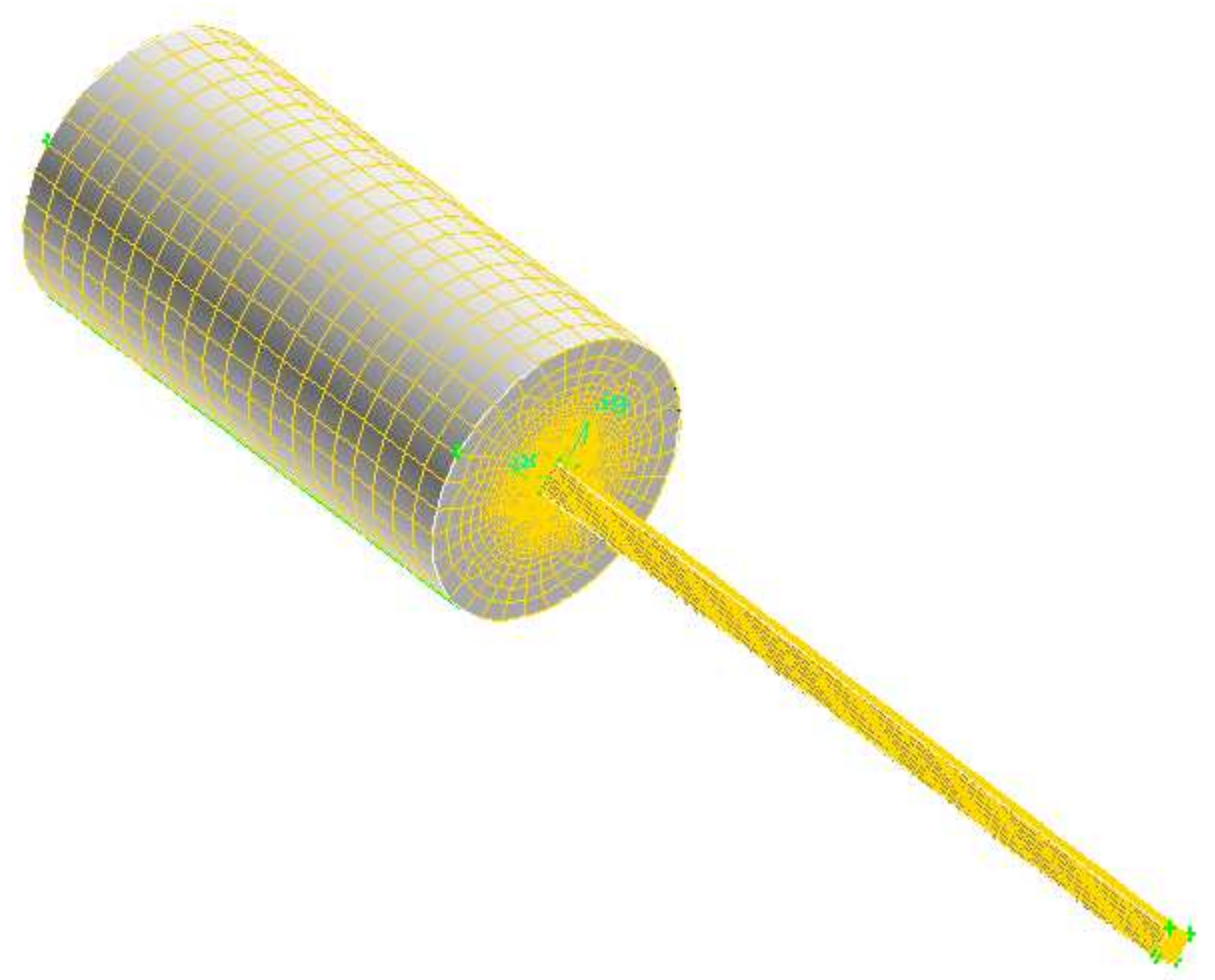

Figure 8-2: 3-D grid of the contraction test section for study with FLUENT.

Table 8-4: Properties at diverse locations of the square lattice honeycomb system for thrust chamber inlet temperature and pressure of $2,800 \mathrm{~K}$ and 500 psi $(=3.45 \mathrm{MPa})$, respectively.

\begin{tabular}{lllll}
\hline Thrust(lbf) & 25,000 & 50,000 & 75,000 & 100,000 \\
\hline Core transferred power(MW) & 512 & 1024 & 1535 & 2045 \\
Mass flow rate (kg/s) & 12.01 & 24.02 & 36.02 & 48.03 \\
Turbo-pump outlet temperature(K) & 27.3 & 29.7 & 32.8 & 37.0 \\
Turbo-pump outlet pressure (psi) & 801 & 1151 & 1627 & 2248 \\
core inlet temperature (K) & 186.4 & 185.7 & 184.5 & 182.9 \\
core inlet pressure (psi) & 622 & 865 & 1148 & 1447 \\
turbine inlet temperature (K) & 192.8 & 194.8 & 197.3 & 200.6 \\
turbine inlet pressure (psi) & 728 & 1074 & 1547 & 2163 \\
Maximum Mach number & 0.13 & 0.25 & 0.36 & 0.46 \\
\hline
\end{tabular}

Table 8-5: Pressure drop across the SLHC core for different densities of foam fuel.

\begin{tabular}{lllllll}
\hline Core Area Flow Fraction (\%) & 17 & 20 & 25 & 30 & 40 & 50 \\
$\Delta \mathrm{P}(\mathrm{Pa})$ & 1181.5 & 872.2 & 552.0 & 365.0 & 171.9 & 87.06 \\
\hline
\end{tabular}




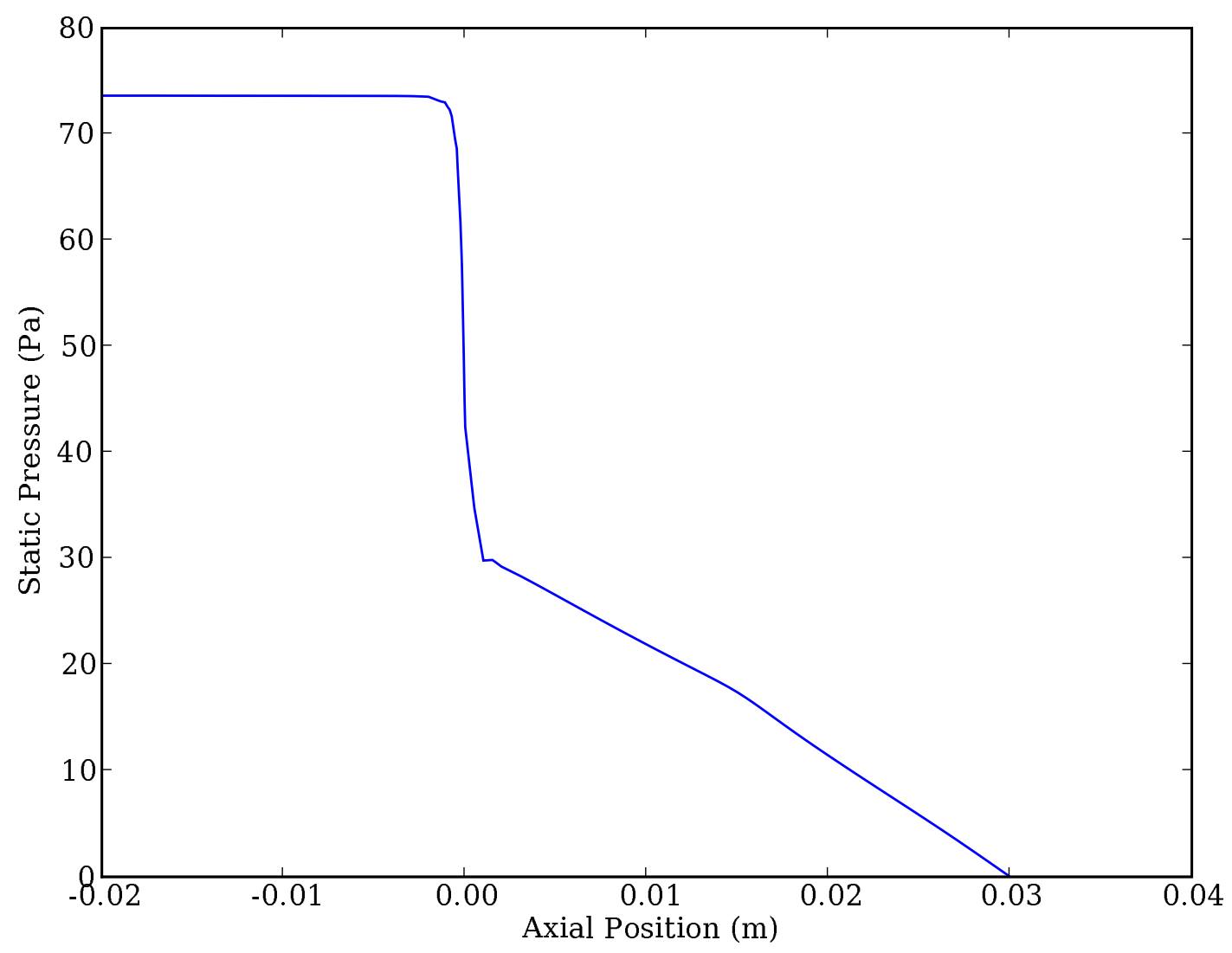

Figure 8-3: Static pressure profile along the axis of the contraction test section

Table 8-6: Comparison of the results obtained with NuRok and Themis

\begin{tabular}{llll}
\hline & NuRok & Themis & relative difference(\%) \\
\hline Pressure drop (Pa) & $3.56 .10^{5}$ & $1.19 .10^{6}$ & 234 \\
Element power density (MW) & 2269.74 & 2626.34 & 15.7 \\
Core inlet temperature (K) & 302.03 & 372.1 & \\
\hline
\end{tabular}




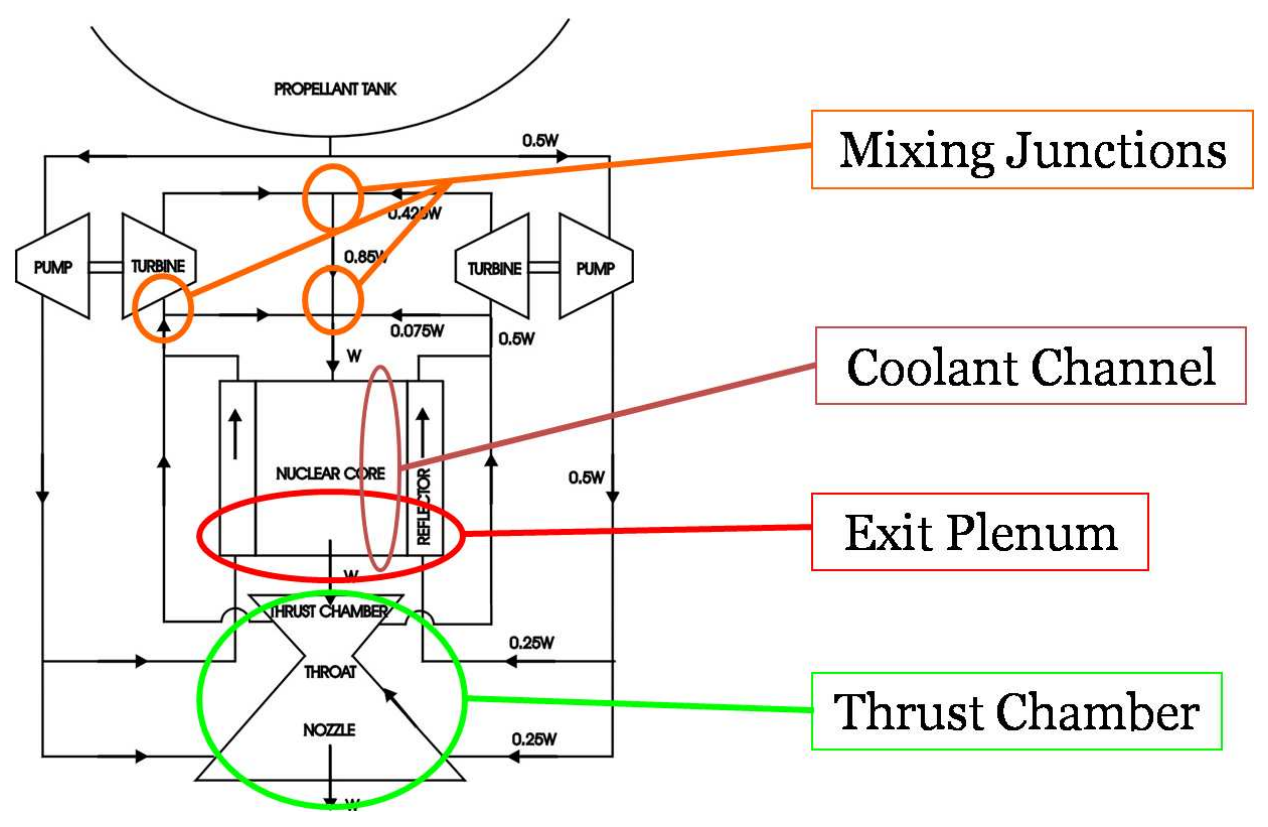

Figure 8-4: Identification and location of portions of the NTP system which would benefit from a more detailed solver 


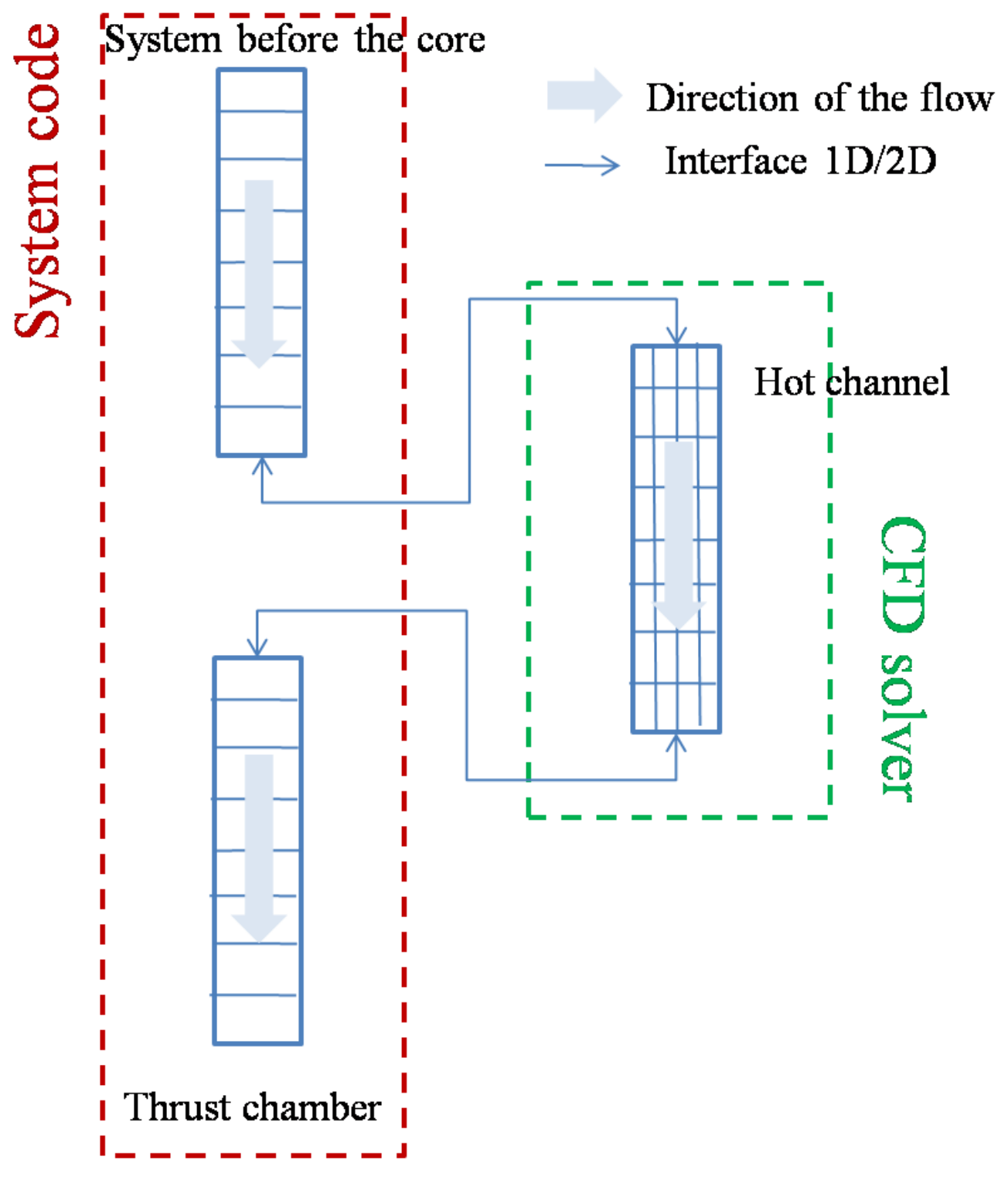

Figure 8-5: Structure of the code coupling when the CFD is used to simulate the hot channel 


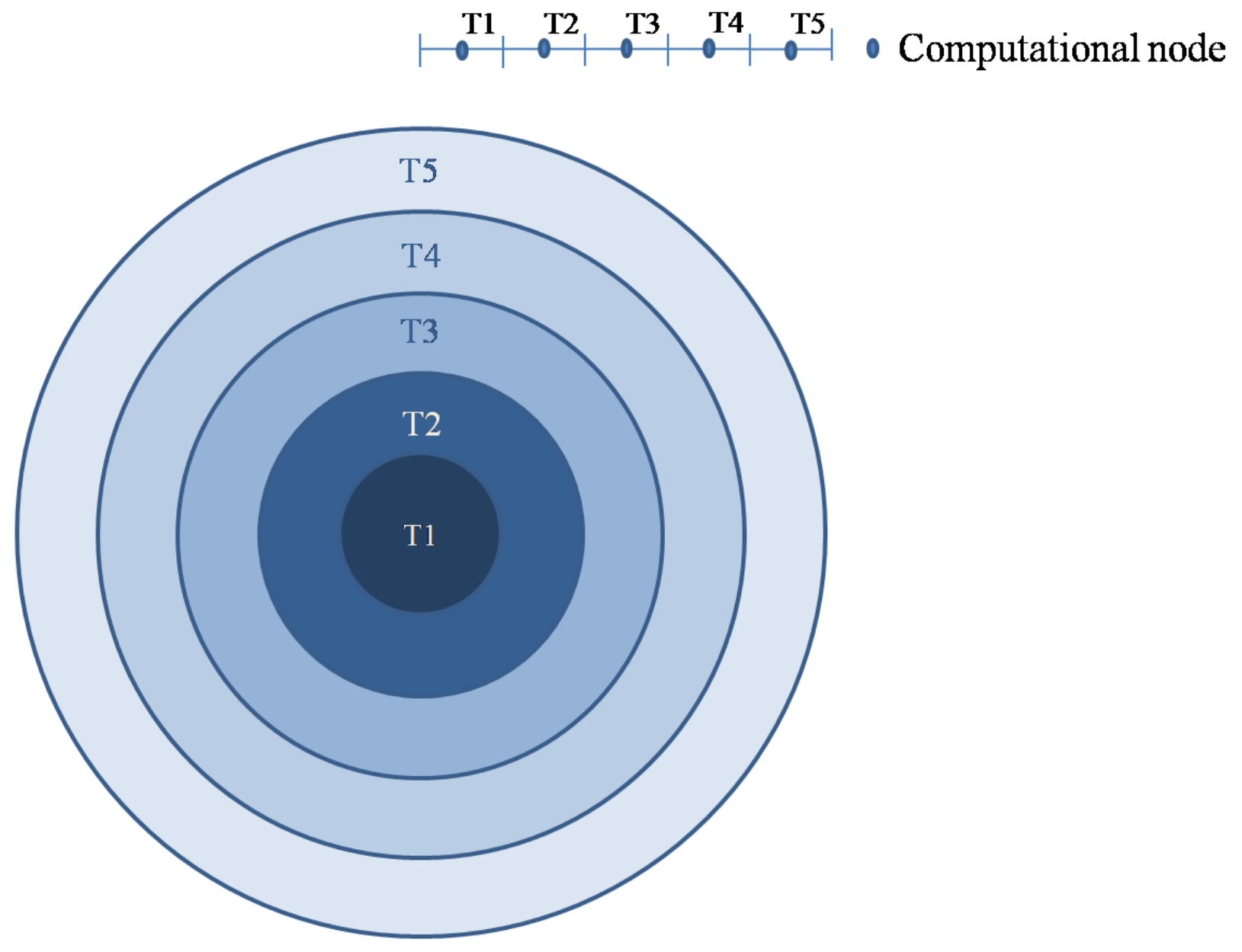

Figure 8-6: Extrapolation of the temperature nodes from the axisymmetric geometry to a single value. 


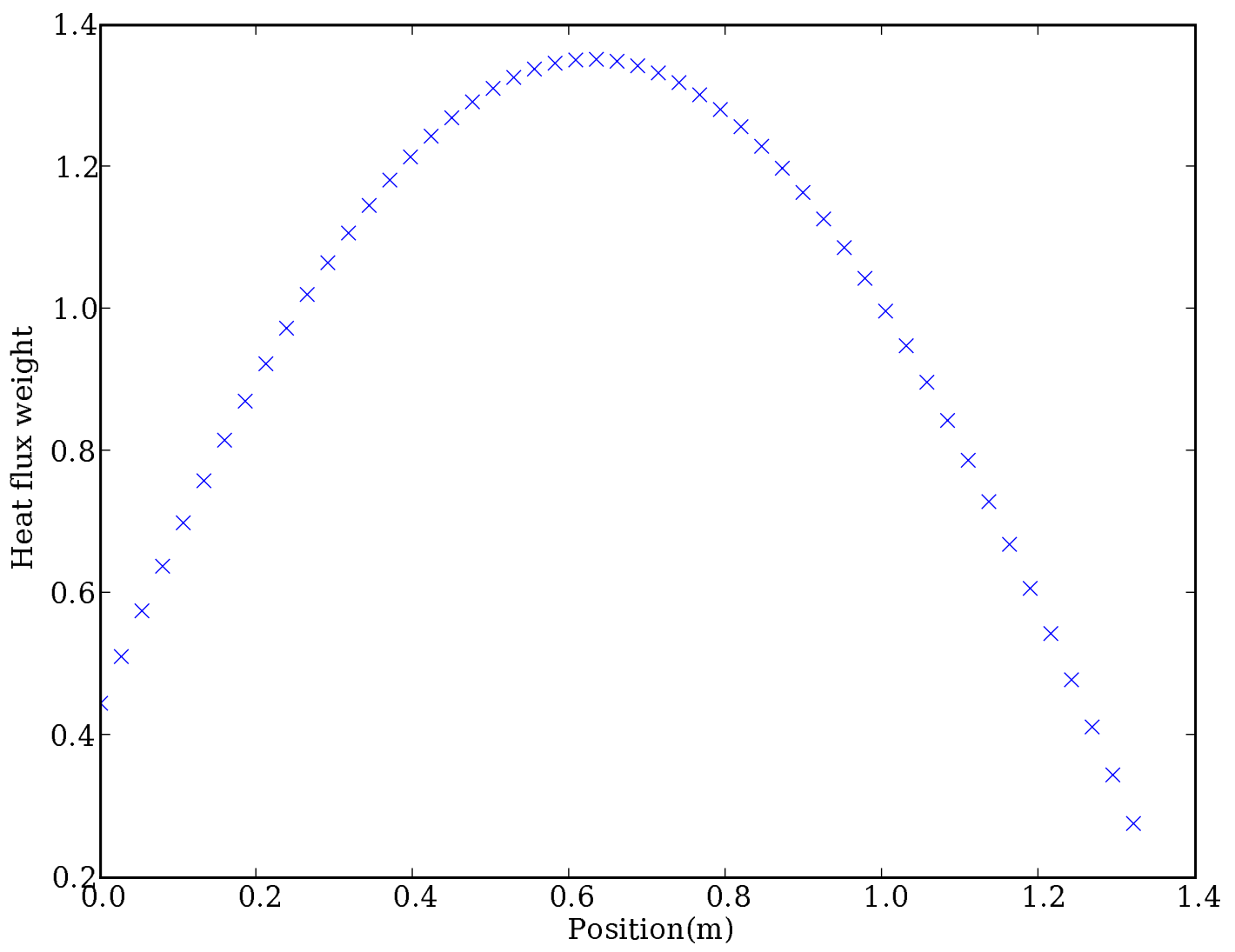

Figure 8-7: Heat profile on the channel walls 


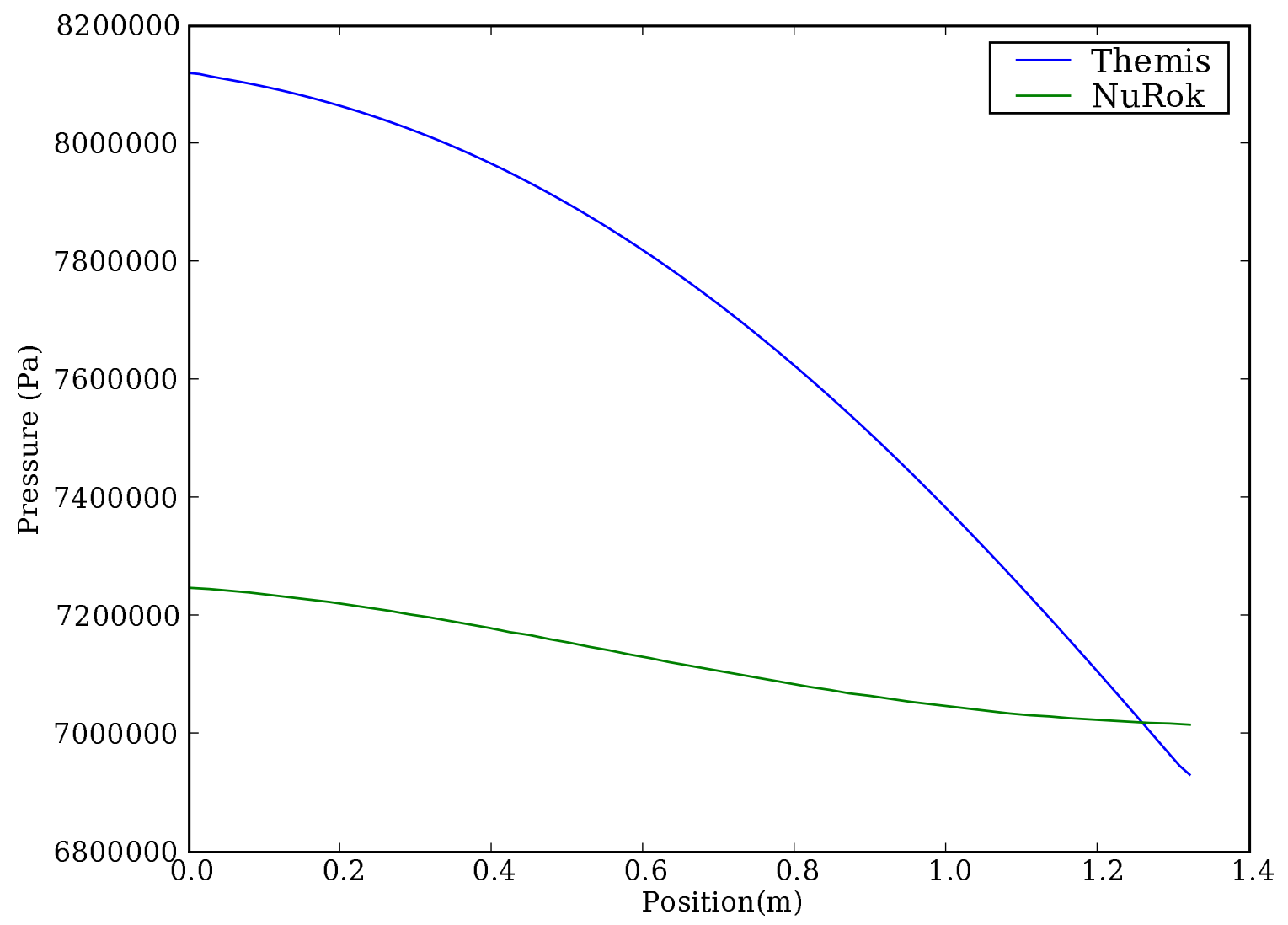

Figure 8-8: Coolant channel pressure profiles calculated with NuRok and Themis 


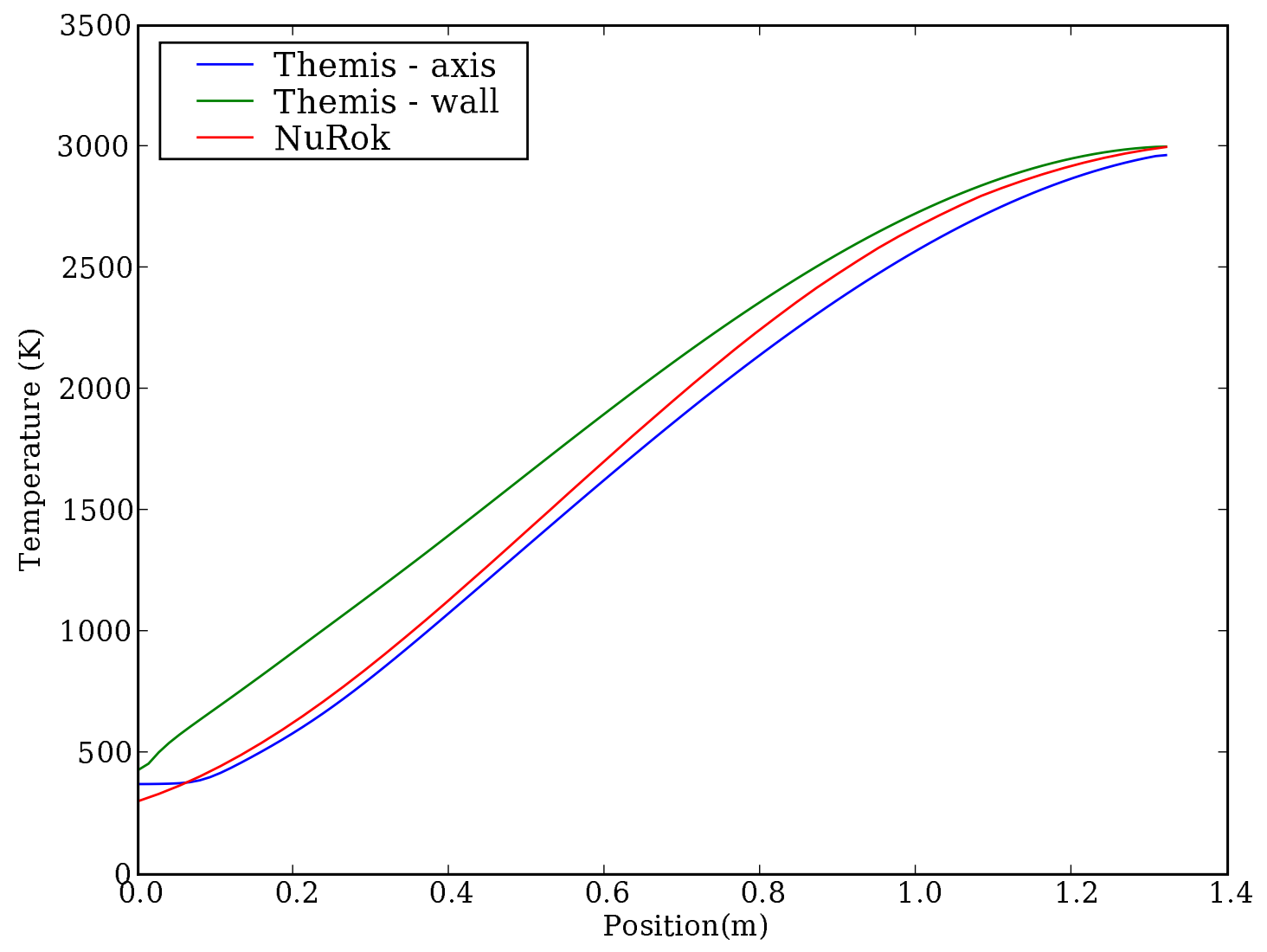

Figure 8-9: Coolant channel temperature profiles calculated with NuRok and Themis (on the axis and the wall) 


\section{CHAPTER 9 \\ CONCLUSIONS AND RECOMMENDATIONS}

The work presented in this dissertation is a contribution to improved accuracy and speed of thermal-fluid simulation tools for high temperature gas cooled reactors. The numerical tools are applied to advanced high temperature nuclear reactors where the coolant and/or the working fluid is a gas such as helium, hydrogen, or carbon dioxide. High temperature gas-cooled reactors are considered for the next generation of nuclear electric power plants as well as for thermochemical production of hydrogen. Another important application of high temperature gas cooled reactors is the nuclear thermal propulsion that is the primary system for powering a future manned mission to Mars. Nuclear thermal rockets due to their high specific impulse and high thrust to weight ration are also considered for many other robotic and manned exploration of the Solar System.

To increase the accuracy and efficiency of the current generation thermal-fluid simulation tools, the technique developed for this work combines the advantages of two commonly used techniques. A system simulation code known for its speed and ability to handle large flow systems is coupled to a fine-mesh CFD solver for which the accuracy and resolution of the calculation are rather high. The fine-mesh code is based on computational fluid dynamics techniques and uses real gas properties. Implementation of real gas property formulation in the fine-mesh CFD solver was motivated by the need to simulate hydrogen flow in a low pressure nuclear thermal propulsion system. At low pressure and high temperatures the hydrogen propellant partially dissociates. Hydrogen dissociation results in reduction of molecular weight that increases the specific impulse. To assess the effect of hydrogen dissociation on specific impulse, a temperature and pressure description of the dissociated hydrogen is needed in the CFD solver. The model should account for the variation of thermal and transport properties as a function of dissociation. Commercial CFD codes lack capability to model temperature and pressure dependent 
hydrogen properties including the molecular weight that plays a key role in the calculation of specific impulse. In this work, the following issues are demonstrated:

1. The feasibility of developing a real property computational fluid dynamics solver to model fluid dynamics and heat transfer of hydrogen and helium cooled high temperature gas cooled systems.

2. The feasibility of coupling a coarse-mesh system simulation code to a fine mesh CFD solver.

The fine-mesh Computational Fluid Dynamics solver developed in this work is directly adaptable to all types of high temperature gas cooled reactors. It consists in a two-dimensional axisymmetric solver that is compatible with the geometry of most high temperature gas cooled reactor systems. The TVD MacCormack solver combined with RANS turbulence models such as $\mathrm{k}-\epsilon$ and Spalart-Allmaras are used to develop a robust CFD solver that incorporates real gas properties. The CFD solver has been applied to the analysis of the coolant channel of a helium cooled High Temperature Gas-Cooled Reactor In the case of the core-length channel the temperature applied on the walls linearly increase from $400 \mathrm{C}$ to $1,000 \mathrm{C}$. The predicted pressure drop across the $7.93 \mathrm{~m}$ channel is $28 \mathrm{kPa}$ Using a simple one-dimensional model the calculated pressure drop in the channel is $443 \mathrm{kPA}$, which is a gross overestimation. The flow in the tube is accelerated such that the exit velocity is twice larger than the inlet velocity. The change in the helium gas pressure and temperature at the core exit result in 47

Then, the coupling of the NuRok coarse-mesh simulation code to the fine mesh CFD solver is done through a simple interfacing procedure which consists of a mathematical interpolation of the extensive properties from 1D code to $2 \mathrm{D}$ axisymmetric code and vice a versa. The coupling is applied to the study of the NERVA derivative nuclear thermal propulsion system. The hot channel in the NERVA core is modeled with the fine mesh CFD solver. The use of the fine mesh CFD solver significantly increases the computational time. However, the total computation time still is less than one minute In the case of a 100,000 lbf thrust NERVA system the pressure drop across the channel is equal to 1.19 
MPa. The coarse mesh simulation alone predicted a pressure drop three times smaller than the values derived by the fine-mesh CFD solver. Therefore, the accuracy of the simulation has been greatly increased by adding the fine mesh simulation of the coolant channel. The computational time remains considerably small since the technique used at the interfaces between the fine- and coarse-mesh solvers leads to fast convergence.

The modeling approach presented in this work is the first attempt in coupling coarseand fine-mesh CFD solvers using real properties of gases. Now, that the basis has been set for such numerical tools, many improvements could be incorporated in the coupled code. Since NuRok is modular, it would be easy to implement fine mesh solvers for other portions of the system which require a detailed thermal-hydraulics analysis. Examples of such areas in the system include mixing junctions or the core inlet and exit plenums. A robust discretization scheme is used to allow for handling of all types of flows such as subsonic, transonic, or supersonic flow. Therefore, the code could be used for simulation of steady state operation as well as for accidental analysis. NuRok could be modified to simulate the entire domain of closed loop High Temperature Gas Cooled systems that would require closing the flow loop and adding a heat exchanger module. A full property package for carbon dioxide is developed. Therefore, the same code could be modified to study supercritical carbon dioxide cooled High Temperature Gas Cooled Reactors.

The development of the real property CFD solver development was motivated by the acknowledgment that in the case of the low-pressure thrust chamber of the NTP, the dissociation process could not be modeled with traditional CFD solvers. The FLUENT study of this particular thrust chamber with frozen chemistry showed great promise as to the performances of the overall system operating at low pressure. The analysis enabled the determination of the converging-diverging nozzle optimum design and estimated the specific impulse of the system to be in the order of 1,400 s at core exit temperature of 3,000 K. This would represent a great increase in performance compared to original NTP designs which traditionally operate at higher pressures with specific impulse of about 925 
s at 3,000 K exit temperature. Modeling of the low-pressure thrust chamber with the real property fine mesh CFD solver remains very challenging because of the sharp variation of the hydrogen heat capacity due to dissociation. Further work should be done to determine a more accurate estimation of the Isp associated with the low pressure nuclear thermal rocket design. In a future work, two approaches could be tested to obtain convergence of the CFD solver with dissociated hydrogen: first, a set of continuous functions could be used to express the hydrogen properties as a function of pressure and temperature. During the first time steps, properties could be coarsely approximated with linear functions and as the solution converges to a steady-state solution, the property formula could be modified to match the real values. The other technique would be to use the look-up tables which were developed for NuRok. This approach is computationally very expensive, however, it may prove to be the only viable option for achieving convergence in cases involving dissociated hydrogen. 


\section{APPENDIX A \\ PROGRAM FOR COMPILATION OF THE EXACT RIEMANN PROBLEM SOLUTION}

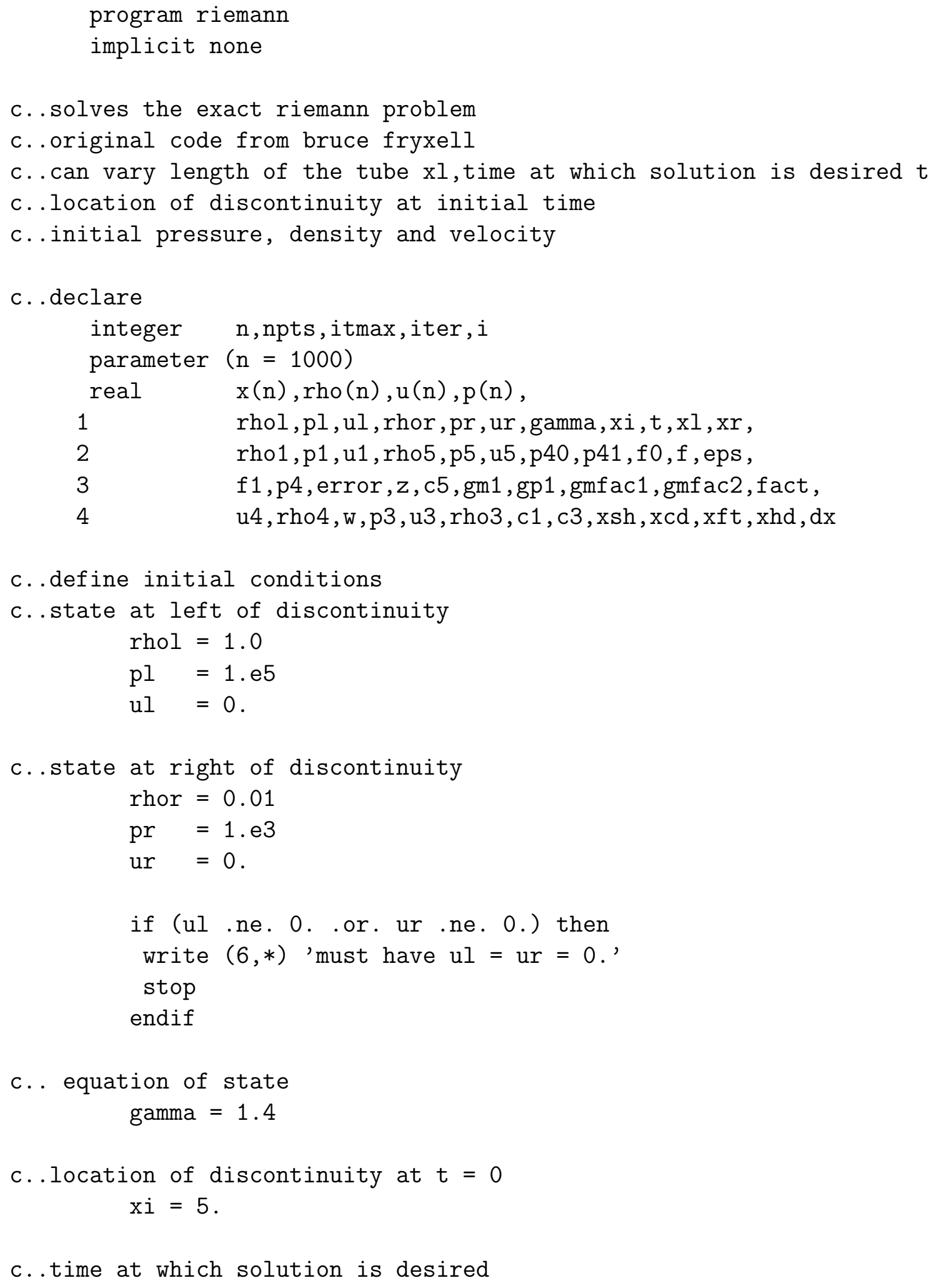




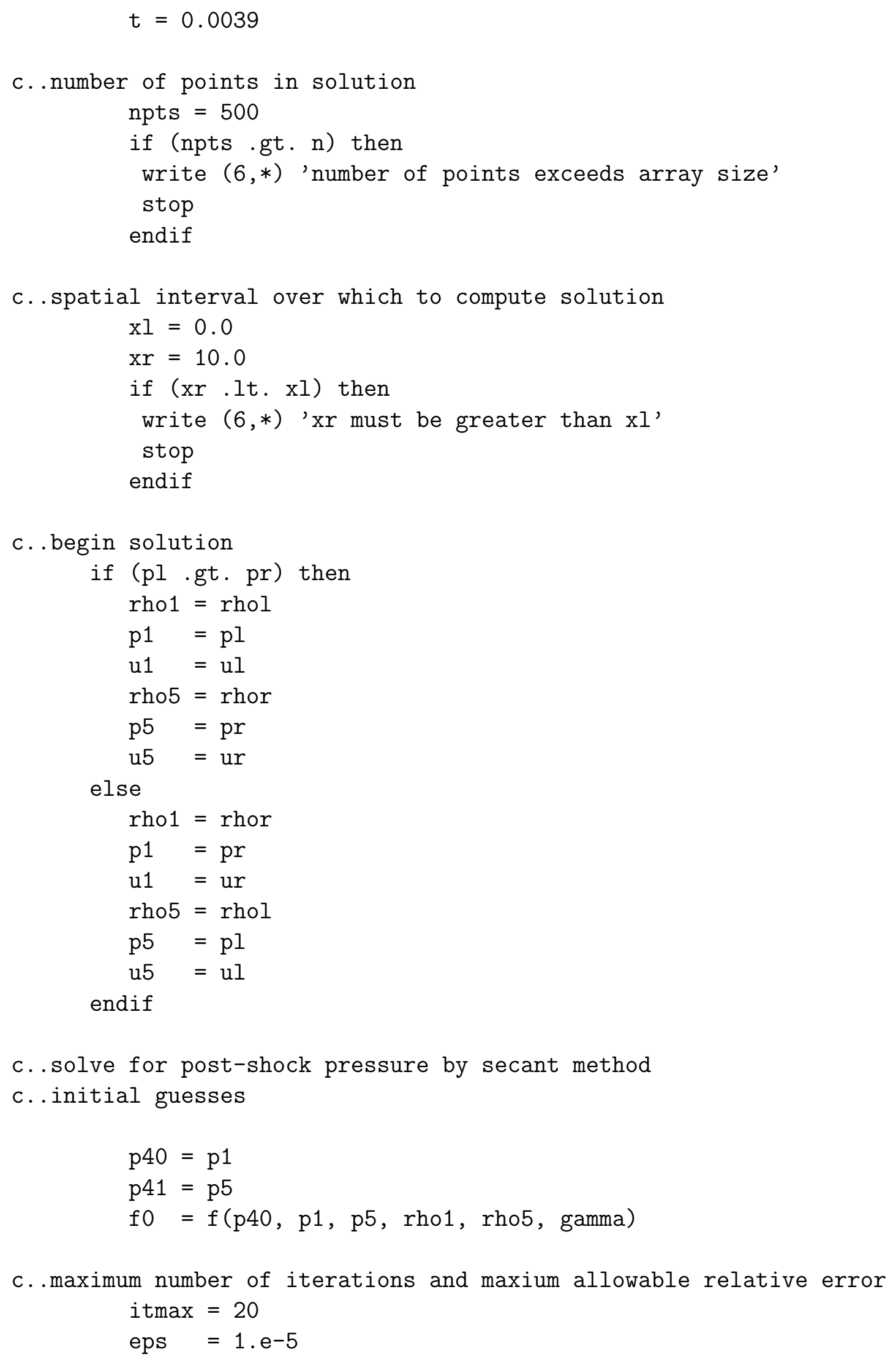




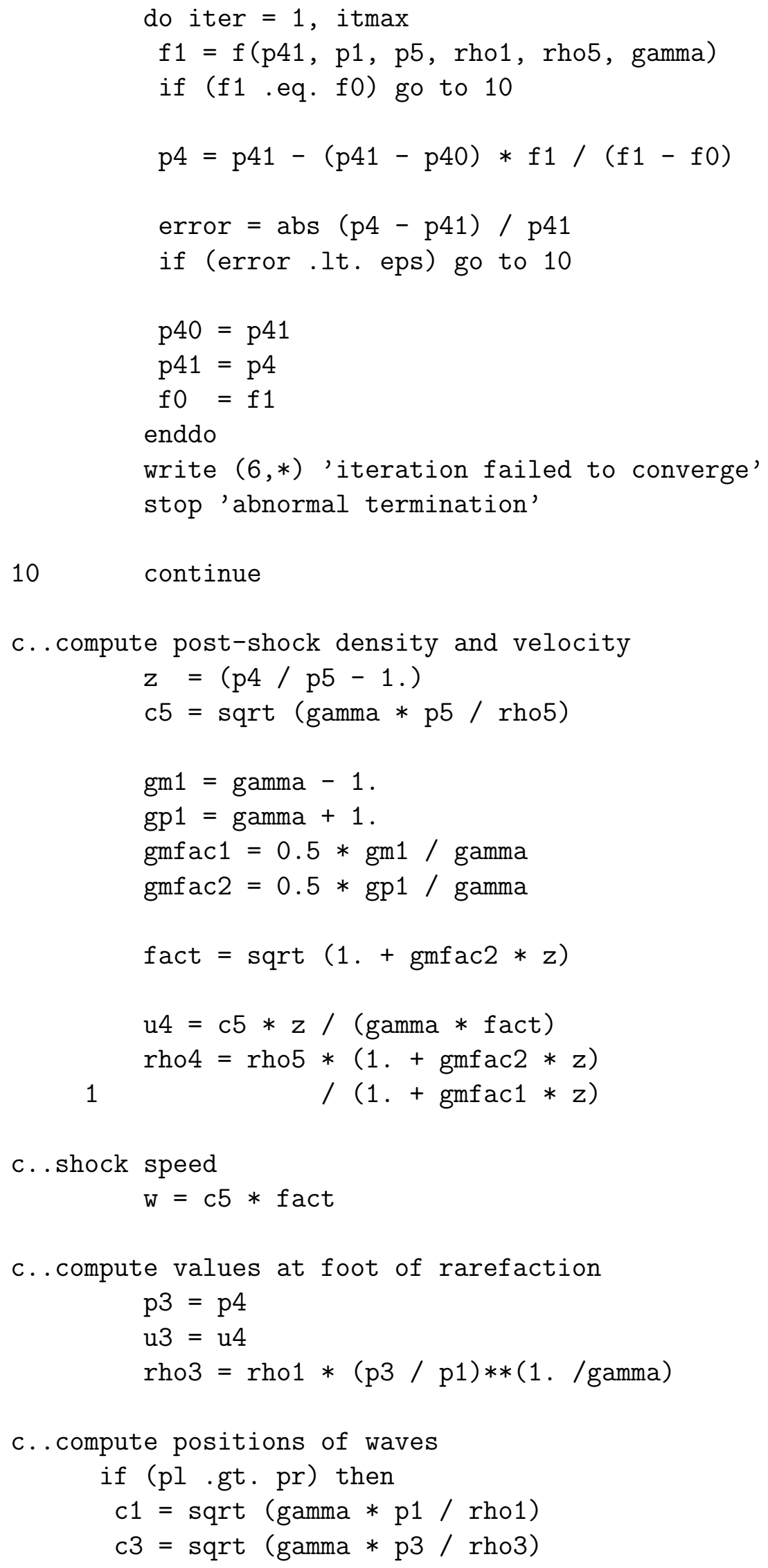




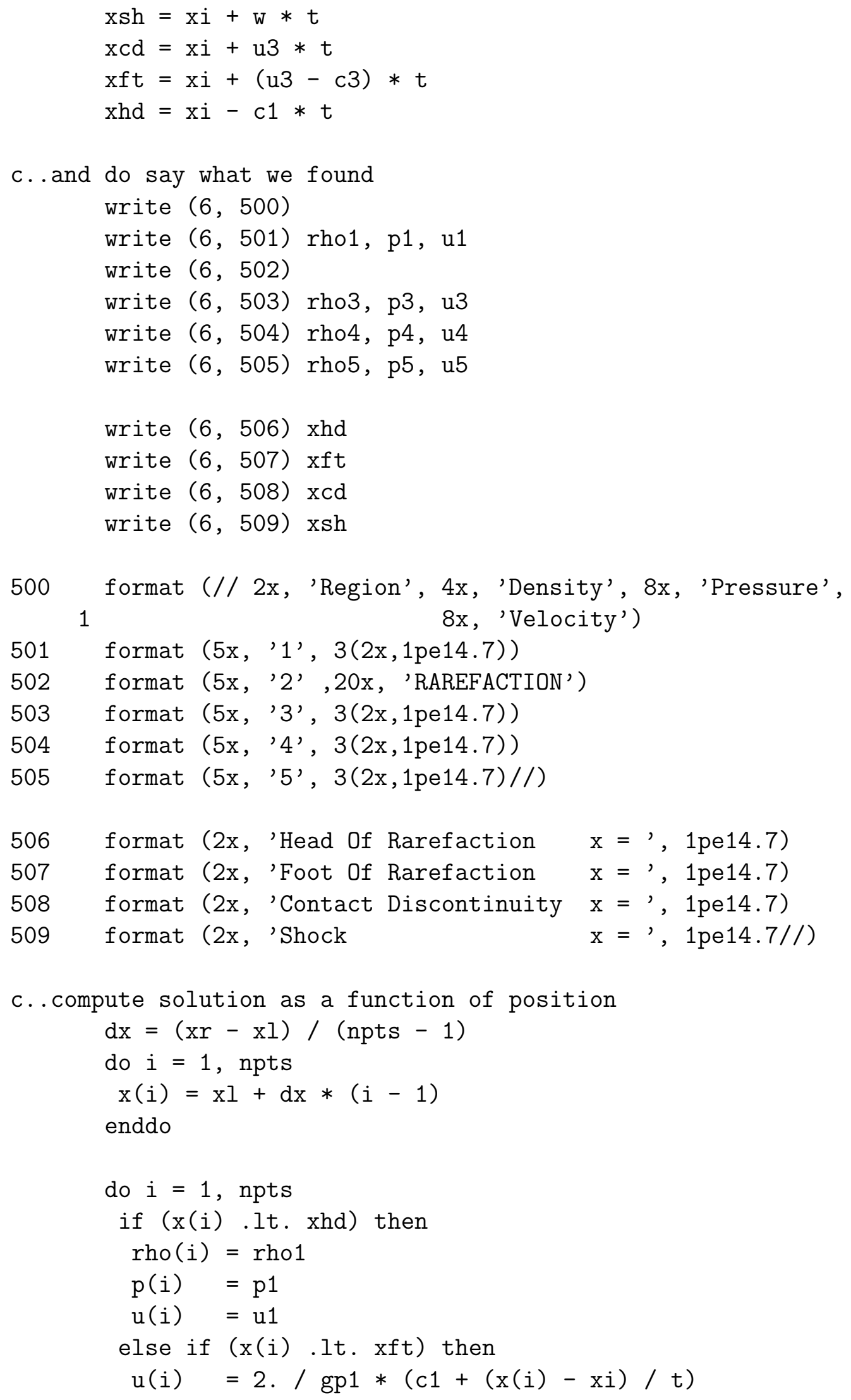




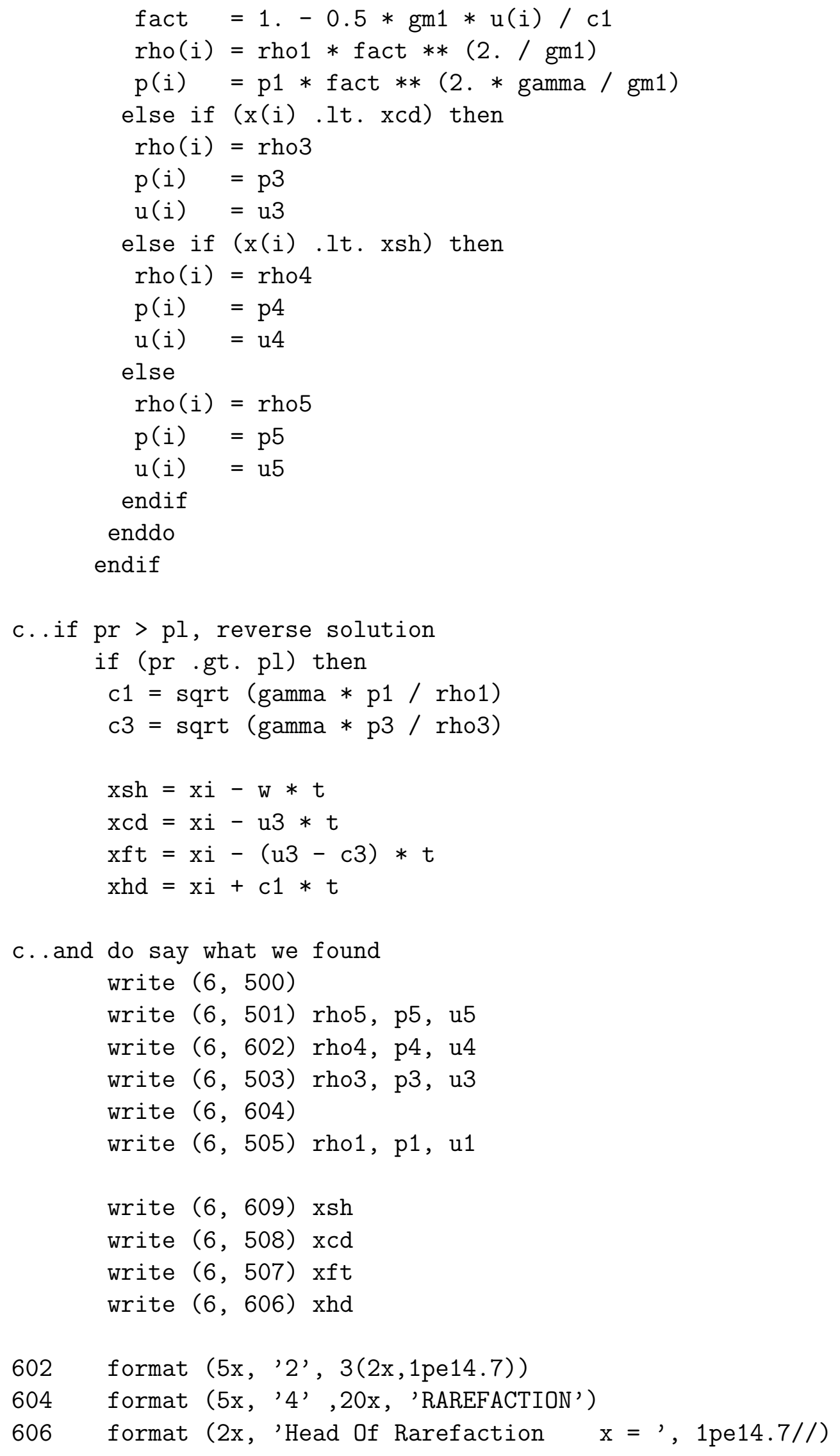




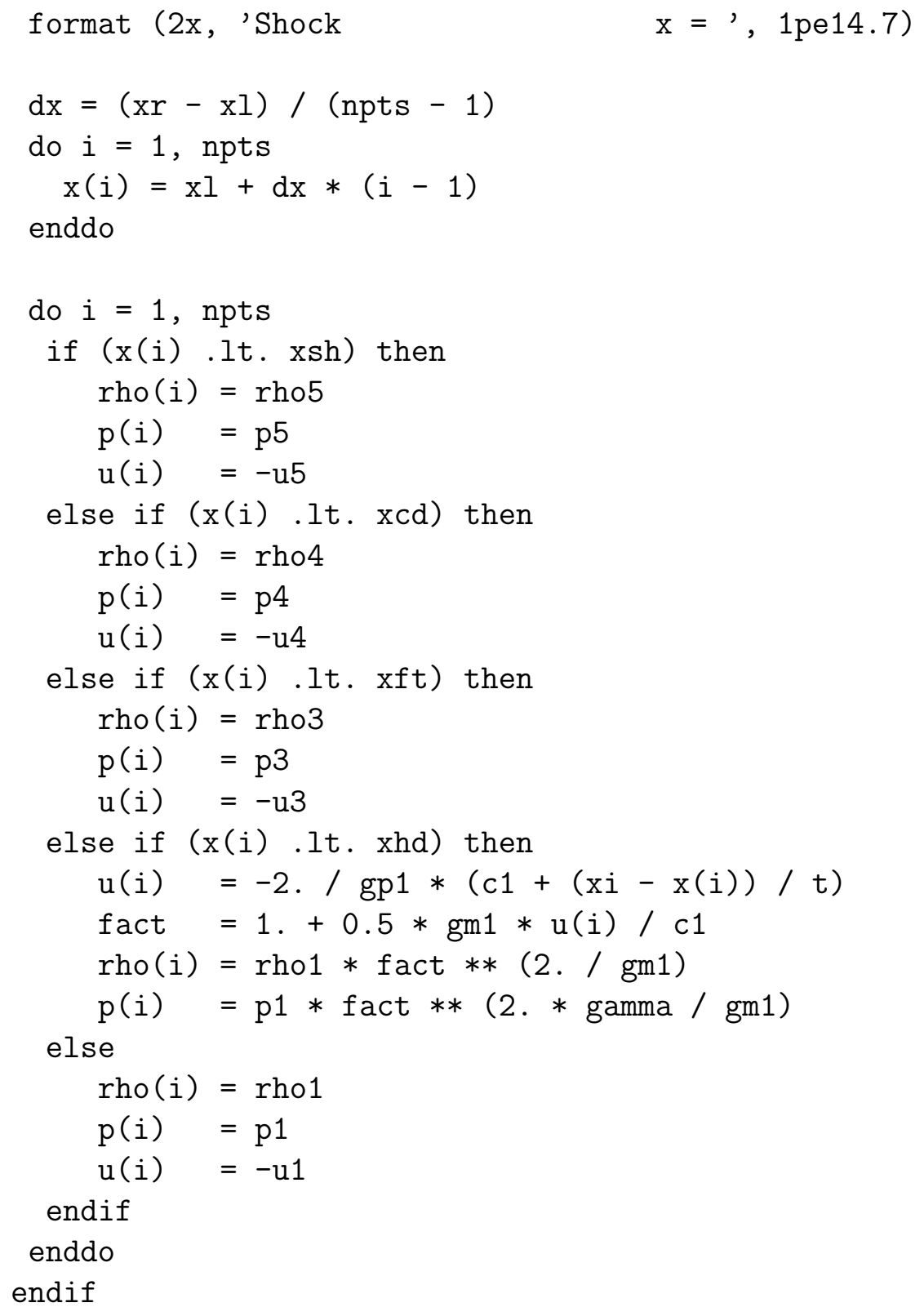


real function $f(p 4, p 1, p 5$, rho1, rho5, gamma)

implicit none

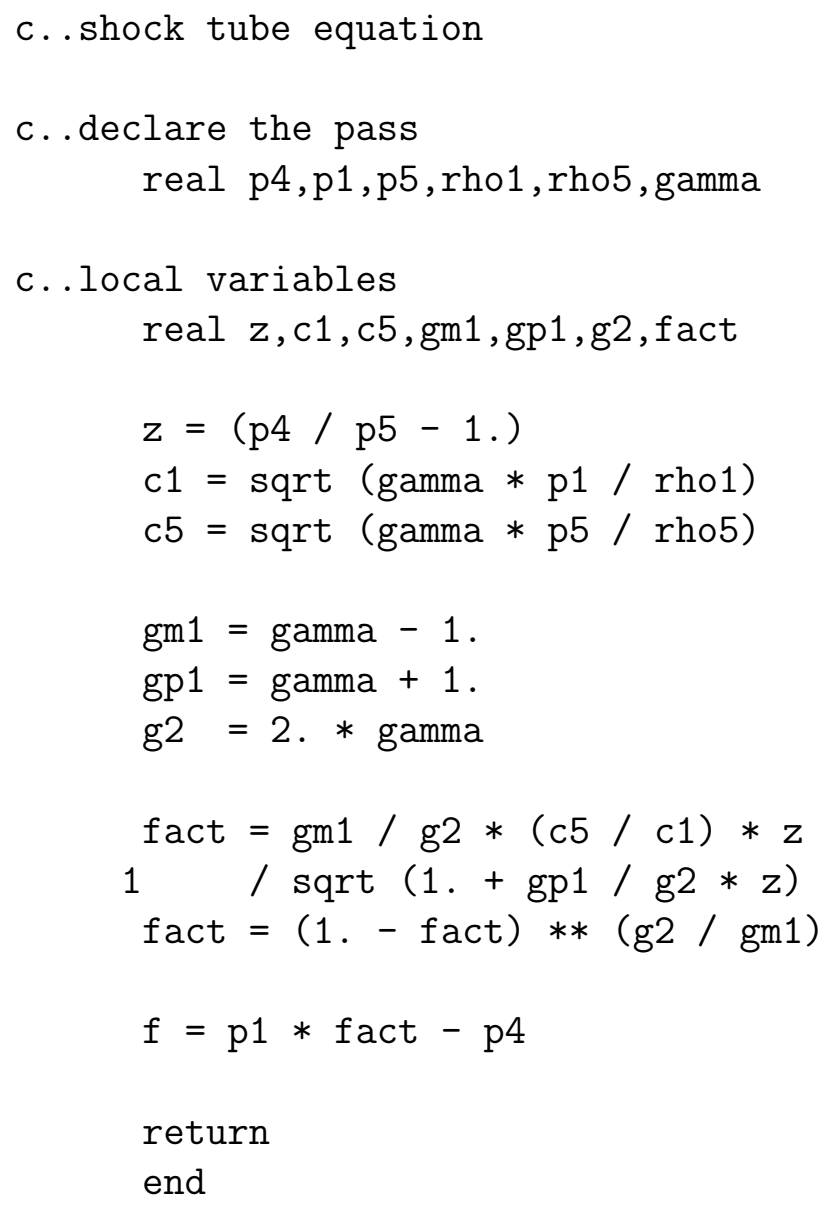




\section{APPENDIX B \\ FLUENT PRELIMINARY ANALYZES}

All preliminary analyzes are performed with FLUENT 6.3.26. The cases we describe here are compatible with any version of FLUENT 6. Definitions of terms particular to the FLUENT solver are given at the end of the appendix.

\section{B.1 Low-Pressure Thrust Chamber: Design Parameter Analysis}

The axisymmetric study is done with a simple 2-D pressure-based (segregated) solver and Spalart-Allmaras model accounts for turbulence. Frozen properties of hydrogen are considered as well as the ideal-gas formulation for density. The operating pressure is set at 0.1 bar $\left(10^{4} \mathrm{~Pa}\right)$. The inlet mass flow rate is equal to $0.228 \mathrm{~kg} / \mathrm{s}$ with the velocity normal to the boundary and the inlet temperature equal to $3,000 \mathrm{~K}$. The turbulent method is initially specified with the turbulent intensity $(10 \%)$ and the hydraulic diameter $(0.6 \mathrm{~m})$. The exit pressure is set to $-9990 \mathrm{~Pa}$, which corresponds to an absolute pressure of $10 \mathrm{~Pa}$. The backflow total temperature is equal to $3,000 \mathrm{~K}$. The discretization scheme is set to a third order MUSCL method for all variables except for pressure for which it is set to a second-order upwind. To improve convergence performances, the pressure is limited to 0.4 bars and the temperature is bounded between 1,500 and 3,500 $\mathrm{K}$. The convergence criteria is set to $10^{5}$.

pressure-based solver and the Spalart-Allmaras model to account for turbulence. Hydrogen properties are given by Equation 7-7and the ideal-gas law describes the density behavior. The inlet mass flow rate is set to $0.228 \mathrm{~kg} / \mathrm{s}$ and the inlet temperature and pressure to 3,000 $\mathrm{K}$ and 0.1 bars respectively. This corresponds to an inlet Mach number of 0.3. The adiabatic behavior is studied, therefore the thermal flux applied to the walls is equal to zero. By default, the walls are considered to be in Aluminum. Finally, the exit pressure is set at $10 \mathrm{~Pa}$. We use a second order solver on pressure and a 3rd order MUSCL on all the other conservative variables. 


\section{B.2 Definitions}

Turbulent intensity: The turbulent intensity is defined by the ratio of the fluctuating velocity over the mean velocity.

$$
I=\frac{u^{\prime \prime}}{\langle u\rangle}
$$

In high turbulence flow, the turbulent intensity usually varies between 5 and 20\%. For fully-developed pipe flows the turbulent intensity can be estimated by:

$$
I \simeq R e_{D_{h}}^{-1 / 8}
$$

where $R e_{D_{h}}$ is the Reynolds number based on the hydraulic diameter $D_{h}$.

Backflow total temperature[6]:The backflow temperature should be input when we use the pressure-outlet boundary condition in FLUENT. The specified values will be used only if flow is pulled in through the outlet. The value might be used in the first few iterations for which FLUENT often computes velocities coming from the exit. Hence, the absolute value of the backflow total temperature itself is not of great importance but should be set with a realistic value to help computation of the first iterations. 


\section{REFERENCES}

[1] J. Duderstadt and L. Hamilton, Nuclear Reactor Analysis, John Wiley \& Sons, 1976.

[2] J. Welty, Fundamentals of Momentum, Heat, and Mass Transfer. 4th ed., Wiley, New York, 2001.

[3] L. Burmeister, Convective Heat Transfer, Wiley, New York, 1993.

[4] "Nuclear power plants in the world - status on 2005-12-31," Tech. Rep., CEA, 2006.

[5] J. Given and S. Anghaie, "A computational model for thermal fluid design analysis of nuclear thermal rockets," Nuclear Technology, vol. 117, pp. 87-108, 1997.

[6] Fluent Inc., Lebanon, NH, FLUENT 6.1 User's Guide, 2006.

[7] A. Charmeau and S. Anghaie, "Thermal-hydraulics analysis of gas core reactors american nuclear society," in Proceedings of NURETH 11, Avignon, France, 2005.

[8] J. Plancher, "Thermal and fluid design analysis of a square lattice honeycomb nuclear rocket engine," Masters thesis, University of Florida, 2002.

[9] T. Knight, S. Anghaie, J. Plancher, and R. Gouw, "Square lattice honeycomb tri-carbide fueled reactor design for space power and propulsion," in Proceedings of International Congress on Advances in Nuclear Power Plants (ICAPP '03)- ANS Publications, Còrdoba, Spain, 2003.

[10] S. Anghaie, G. J. Feller, S. D. Peery, and R. C. Parsley, "A fast spectrum dual path flow cermet reactor," 1993, vol. 271, pp. 663-668.

[11] P. A. Tipler, Elementary Modern Physics, vol. Chap. 5, W. H. Freeman, 1992.

[12] N. Vargaftik, Handbook of Physical Properties of Liquids and Gases, Hemisphere Publishing, New York, 1995.

[13] A. W. Date, Introduction to Computational Fluid Dynamics, Cambridge University Press, Cambridge, 2005.

[14] A. Inc., Ansys CFX versions 5.7, Users Manual, 2004.

[15] CD-adapco, StarCD 3.0 Reference Manual, London, 1998.

[16] C. Freitas, "Perspective: Selected benchmarks from commercial cfd codes," Journal of Fluid Engineering, vol. 117, pp. 208-218, 1995.

[17] B. Launder and D. Spalding, Mathematical models of turbulence, Academic Press, 1972.

[18] G. Yadigaroglu, M. Andreani, J. Dreier, and P. Coddington, "Trends and needs in experimentation and numerical simulation for lwr safety," Nuclear Engineering and Design, vol. 221, no. 1-3, pp. 205-223, April 2003. 
[19] C. Boyd and K. Hardesty, "Cfd analysis of 1/7th scale steam generator inlet plenum mixing during a pwr severe accident," Tech. Rep. NUREG-1781, U.S. Nuclear Regulatory Commission, 2003.

[20] C. Boyd, D. Helton, and K. Hardesty, "Cfd analysis of full-scale steam generator inlet plenum mixing during a pwr severe accident," Tech. Rep. NUREG-1788, U.S. Nuclear Regulatory Commission, 2004.

[21] T. Keheley, "Modeling a boron dillution transient in a pressurized water reactor," Star-CD Dynamics, vol. 21, pp. 24, 2003.

[22] H. Tinoco, "Three-dimensional modeling of a steam-line break in a boiling water reactor," Nuclear Science and Engineering, vol. 140, pp. 152-164, February 2002.

[23] K. Rehme, "The structure of turbulence in rod bundles and the implications on natural mixing between the subchannels," Int. J. Heat Mass Transfer, vol. 35, pp. $567,1992$.

[24] J. Hooper and D. Wood, "Fully-developed rod bundle flow over a large range of reynolds number," Nuclear Engineering and Design, vol. 83, pp. 31, 1984.

[25] C. Tzanos, "Computational fluid dynamics of light water reactor flows," Nuclear Technology, vol. 147, pp. 181, 2004.

[26] W.-K. In and T.-H. Chun, "Assessment of the rans turbulence models for a turbulent flow and heat transfer in a rod bundle," Nuclear Technology, vol. 150, pp. $231,2005$.

[27] W.-K. In, D.-S. Oh, and T.-H. Chun, "Simulation of turbulent flow in rod bundles using eddy viscosity models and the reynolds stress model," in Proceedings of NURETH10, Seoul, Korea, 2003.

[28] D. Bestion, "Recommendation on use of cfd codes for nuclear reactor safety analysis," Tech. Rep. FIKS-CT-2001-00154, European Commission, 2004.

[29] R. Schultz and D. Schowater, "Cfd for advanced nuclear reactor design," Fluent News, vol. XIII, no. 2, 2004.

[30] D. M. McEligot and G. E. McCreery, "Scaling studies and conceptual experiment designs for ngnp cfd assessment," Tech. Rep. INEEL/EXT-04-02502, Idaho National Laboratory, 2004.

[31] I. Ahmad, K.-Y. Kim, W.-J. Lee, and G.-C. Park, "Numerical study on flow field in inlet plenum of a pebble-bed modular reactor," Nuclear Engineering and Design, vol. 237, no. 6, pp. 565-574, March 2007.

[32] S. Lee, "Heat transfer modeling of dry spent nuclear fuel storage facilities," in Proceedings of the 33rd National Heat Transfer Conference, Albuquerque, NM, 1999. 
[33] D. P. Weber, T. Wei, R. A. Brewster, D. T. Rock, and Rizwan-uddin, "High fidelity thermal-hydraulic analysis using cfd and massively parallel computers," Tech. Rep. ANL/RE/CP-101101, University of Chicago and Argonne National Laboratory, 2000 .

[34] S. H. Yoon and K. Suh, "Analysis of direct vessel injection flow patern using cfx code," in ANS Transactions, 1999, vol. 81, pp. 334-335.

[35] W. Weaver, E. Tomlinson, and D. Aumiller, "A generic semi-implicit coupling methodology for use in relap-3d," Nuclear Engineering and Design, vol. 211, pp. $13-26,2002$.

[36] C. Chauliac, J. Aragonés, D. Bestion, D. Cacuci, P. Coddington, and L. Dada, "A european platform for nuclear reactor simulation," in ICONE-14 Workshop on advanced LWR, Miami, USA, July 2006.

[37] M. Scheuerer, "Evaluation of computational fluid dynamics methods for reactor safety analysis - condensed final summary report," Tech. Rep. EVOL ECORA D 17, European Commission, 2005.

[38] C. Hirsch, Numerical computation of internal and external flows, vol. 1\& 2, John Wiley \& Sons, 1989 \& 1990.

[39] R. Courant, E. Isaacson, and M. Reeves, "On the solution of nonlinear hyperbolic differential equations by finite difference," Comm. Pure and Applied Mathematics, vol. 5, pp. 243-255, 1952.

[40] P. Lax, "Weak solutions of non linear hyperbolic equations and their numerical computation," Comm. Pure and Applied Mathematics, vol. 7, pp. 159-193, 1954.

[41] R. Fedkiw, G. Sapiro, and C.-W. Shu, "Shock capturing, level sets, and pde based methods in computer vision and image processing: a review of osher's contributions," Journal of Computational Physics, vol. 185, pp. 309341, 2003.

[42] P. Lax and B. Wendroff, "Systems of conservation laws," Comm. Pure. Appl. Math., vol. 13, pp. 217237, 1960.

[43] R. W. MacCormack, "The effect of viscosity in hypervelocity impact cratering," in AIAA Hypervelocity Impact Conference, Cincinnati, OH, 1969.

[44] S. Godunov, "A difference method for the numerical calculation of discontinuous solutions of hydrodynamic equations," Mat. Sbornik, vol. 47, pp. 271306, 1959, Translated as JPRS 7225 by U.S. Dept. of Commerce - 1960.

[45] D. A. Caughey and A. Jameson, "Development of computational techniques for transonic flows: An historical perspective," in Symposium Transsonicum IV, International Union of Theoretical and Applied Mechanics, DLR Gottingen, Germany, September 2-6 2002. 
[46] P. Roe, "Approximate riemann solvers, parameter vectors and difference schemes," Journal of Computational Physics, vol. 43, pp. 357-372, 1981.

[47] S. Osher, Numerical Methods for Fluid Dynamics, chapter Shock modelling in aeronautics, pp. 179-218, Academic Press, London, 1982.

[48] S. Chakravarthy, A. Harten, and S. Osher, "Essentially non-oscillatory shock capturing schemes of uniformly very high accuracy," in AIAA 24th Aerospace Sciences Meeting, Reno, Nevada, 1986, number AIAA Paper 86- 0339.

[49] J. Steger and R. Warming, "Flux vector splitting of inviscid gas-dynamic equations with applications to the finite difference method," Journal of Computational Physics, vol. 40, pp. 263-293, 1981.

[50] B. V. Leer, "Towards the ultimate conservative difference scheme. v. a second order sequel to godunov's method," Journal of Computational Physics, vol. 32, pp. 101-136, 1979.

[51] J. Boris and D. Book, "Flux corrected transport, 1 shasta, a fluid transport algorithm that works," Journal of Computational Physics, vol. 11, pp. 3869, 1973.

[52] A. Harten, "High resolution schemes for hyperbolic conservation laws," Journal of Computational Physics, vol. 49, no. 357393, 1983.

[53] A. Jameson, "Solution of the euler equations by a multigrid method," Applied Mathematics and Computations, vol. 13, pp. 327356, 1983.

[54] D. Wilcox, Turbulence Modeling for CFD, DCW Industries, Inc, La Canada, CA, 1993.

[55] R. W. MacCormack and B. S. Balwin, "A numerical method for solving the navier-stokes equations with application to shock-boundary layer interactions," in AIAA 13th Aerospace Sciences Meeting, Pasadena, CA, 1975.

[56] R. MacCormack, "A numerical method for solving the equations of compressible viscous flow," AIAA Journal, vol. 20, no. 9, pp. 1275-1281, 1982.

[57] S. L. Lawrence, J. C. Tannehill, and D. S. Chaussee, "Application of the implicit maccormack scheme to the parabolized navier-stokes equations," AIAA Journal, vol. 22, no. 12, pp. 1755-1763, 1984.

[58] R. D. Snyder and J. N. Scott, "Comparison of numerical schemes for the analysis of aeroacoustics," in 37th AIAA Aerospace Sciences Meeting and Exhibit, Reno, NV, 1999, number AlAA 99-0354.

[59] R. MacCormack and B. Baldwin, "A numerical method for solving the navier-stokes equations with applications to shock boundary layer intersections," in 13th AIAA Aerospace Meeting, Pasadena, CA, 1975, number AIAA paper 75-1. 
[60] S. Obayashi and K. Kuwahara, "Lu factorization of an implicit scheme for the compressible navier-stokes equations," AIAA Journal, , no. AIAA Paper 84-1670, 1984.

[61] C. Ong and D. Knight, "Hybrid maccormack and implicit beam-warming algorithms for a supersonic compression corner," AIAA JOURNAL, vol. 25, no. 3, pp. 401-407, 1987.

[62] R. Warming and R. Beam, "Upwind second order difference schemes and applications in aerodynamics flows," AIAA Journal, vol. 14, pp. 1241-1249, 1976.

[63] B. Baldwin and H. Lomax, "Thin layer approximation and algebraic model for separated turbulent flows," AIAA Paper 78-25\%, 1978.

[64] J. Anderson, Computational Fluid Dynamics, McGraw-Hill Science, 1995.

[65] H. Versteeg and W. Malalasekera, An Introduction to Computational Fluid Dynamics The Finite Volume Method, Pearson Prentice Hall, London, 1995.

[66] A. Kolmogorov, "Local structure of turbulence in incompressible viscous fluid flow for very large reynolds number," Doklady, AN. SSR,, vol. 30, pp. 199, 1941.

[67] L. Davidson, "Hybrid les-rans : A combination of a one-equation sgs model and a k-omega model for predicting recirculating flows," in European Congress on Computational Methods in Applied Sciences and Engineering (ECCOMAS), Swansea, Wales, 2001.

[68] N. J. Georgiadis, J. I. D. Alexander, and E. Reshotko, "Development of a hybrid rans/les method for compressible mixing layer simulations," Technical Memorandum TM2001-210762, National Aeronautics and Space Agency, 2001.

[69] P. Spalart and S. Allmaras, "A one-equation turbulence model for aerodynamic flows," Technical Report AIAA-92-0439, American Institute of Aeronautics and Astronautics, New York, 1992.

[70] B. Gatski and C. Rumsey, "Linear and nonlinear eddy viscosity models," in Closure Strategies for Turbulent and Transitional Flows, B. Launder and N. Sandham, Eds., pp. 9-46. Cambridge University Press, 2002.

[71] R. Paciorri, W. Dieudonne, G. Degrez, J.-M. Charbonnier, and H. Deconinck, "Exploring the validity of the spalartallmaras turbulence model for hypersonic flows," Journal of Spacecraft and Rockets, vol. 35, no. 2, pp. 121-126, March-April 1998.

[72] N. Frink and S. Pirzadeh, "Tetrahedral finite-volume solutions to the navier-stokes equations on complex configurations," in Tenth International Conference on Finite Elements in Fluids, Tucson, AZ, January 1998. 
[73] A. Bonfiglioli, P. D. Palma, G. Pasczio, and M. Napolitano, "An implicit fluctuation splitting scheme for turbomachinery flows," Journal of Turbomachinery, vol. 127, pp. 395-401, 2005.

[74] R. H. Cavicchi, "A three-dimensional cfd investigation of secondary flow in an accelerating, 90 elbow," Technical Memorandum TM2001-211219, National Aeronautics and Space Administration, 2001.

[75] D. W. Schwenke, "A theorical prediction of hydrogen molecule dissociation-recombination rates including an accurate treatment of internal state nonequilibrium effects," Journal of Chemical Physics, vol. 92, no. 12, pp. 7267-7282, June 1990.

[76] J. Walton, "Computer program for thermal and transport properties of parahydrogen from 20 to 10 000k," NASA Technical Paper 3378, 1993.

[77] G. Chen, "A computational model for convective, conductive and radiative heat transfer in high power density gas-cooled and gaseous core nuclear reactors," Masters thesis, University of Florida, 1995.

[78] J. Given, "Engine system simulation and core thermo-fluid dynamics of nuclear thermal propulsion rockets," Masters thesis, University of Florida, 1993.

[79] E. Lemmon, M. McLinden, and D. Friend, "Thermophysical properties of fluid systems," NIST Chemistry WebBook, NIST Standard Reference Database Number 69 20899, National Institute of Standards and Technology, Gaithersburg MD, June 2005.

[80] F. Drullion, Définition et étude de systmes linéaires pour la simulation d'écoulement et l'optimisation de formes par méthode de gradient, Ph.d dissertation, Ecole doctorale de Mathématiques et Informatique de Bordeaux, 2004.

[81] G. Lehanasch, Contribution à l'Etude Numérique des Jets Supersoniques SousDétendus, Phd dissertation, Université de Poitiers, 2005.

[82] A. Smith and T. Cebeci, "Numerical solution of the turbulent boundary layer equations," report DAC 33735, Douglas aircraft division, 1967.

[83] L. Prandtl, "Uber ein neues formelsysytem fur die ausgebiildete turbulenz," Nachr. Akad. Wiss Gottingen, pp. 6-19, 1945.

[84] B. Spalding, Ed., Kolmogorov's Two-Equation Model of Turbulence, vol. 434 of Turbulence and Stochastic Process: Kolmogorov's Ideas 50 Years On. Mathematical and Physical Sciences, 1991.

[85] B. Launder and D. Spadling, "The numerical computation of turbulent flows," Comput. Methods Appl. Mech. Eng., vol. 3, pp. 269, 1974. 
[86] D. Wilcox, "Reassessment of the scale-determining equation for advanced turbulence models," AIAA Journal, vol. 26, no. 11, pp. 1299-1310, 1988.

[87] R.Paciorri, "Implementation and validation of the spalart-allmaras turbulence model for applications in hypersonic flows," Technical note, Von Karman Institute for Fluid Dynamics, Rhode Saint Genese, Belgium, 1996.

[88] E. Lorin, A. B. H. Ali, and A. Soulaimani, "An accurate positivity preserving scheme for the spalart-allmaras turbulence model," in European Congress on Computational Methods in Applied Sciences and Engineering, Jyvaskyla, Finland, 2004.

[89] B. Mohammadi and J. Saiac, "Turbulent compressible axisymmetric flows computation with the k-epsilon model," Research Report 1764, Institut National de Recherche en Informatique et en Automatique, Le Chesnay, France, October 1992.

[90] P. R. M. Lyra, R. de C. F. de Lima, D. K. E. de Carvalho, and G. M. L. L. da Silva, "An axisymmetric finite volume formulation for the solution of heat conduction problems using unstructured meshes," J. of the Braz. Soc. of Mech. Sci. \& Eng., vol. XXVII, no. 4, pp. 407-414, 2005.

[91] A. Guardone and L. Vigevano, "Finite element/volume solution to axisymmetric conservation laws," J. Comput. Phys., vol. 224, no. 2, pp. 489-518, 2007.

[92] J. Fürst, Numerical modeling of the transonic flows using TVD and ENO schemes, Phd thesis, CVUT v Praze and l'Universite de la Mediterranee, Marseille, 2000.

[93] S. Anghaie and G. Chen, "A computational fluid dynamics and heat transfer model for gaseous core and very high temperature gas-cooled reactors," Nuclear Science and Engineering, vol. 130, 1998.

[94] D. M. Causon, "High resolution finite volume schemes and computational aerodynamics," in Nonlinear Hyperbolic Equations - Theory, Computation Methods and Applications, J. Ballmann and R. Jeltsch, Eds., pp. 63-74. Notes on Numerical Fluid Mechanics, Braunschweig, Germany, 1989.

[95] R. Vilim, E. Feldman, W. Pointer, and T. Wei, "Initial vhtr accident scenario classification: Models and data," Status report, Nuclear Engineering Division Argonne National Laboratory, 9700 S. Cass Avenue - Argonne IL 60439, Septembre 2004.

[96] D. T. I. et. al, "Status of preconceptual design of the advanced high-temperature reactor (ahtr)," Tech. Rep. ORNL/TM-2004/104, Oak Ridge National Laboratory, Oak Ridge, TN, May 2004.

[97] P. Oosthhuizen and W. Carscallen, Compressible Fluid Flow, McGraw Hill International Editions, Singapore, 1997. 
[98] G. Rao, J. Beck, and T. Booth, "Nozzle optimization for space-based vehicles," in 35th AIAA/ASME/SAE/ASEE Joint Propulsion Conference and Exhibit, Los Angeles, CA, 1999, number AIAA 99-2584.

[99] J. Korte, "Inviscid design of hypersonic wind tunnel nozzle for a real gas," in 38th Aerospace Sciences Meeting and Exhibit-AIAA 2000-0677, Reno, NV, 2000.

[100] M. M. Hussaini and J. Korte, "Investigation of low-reynolds number rocket nozzle design using pns-based optimization procedure," Tech. Rep. NASA Technical Memorandum 110295, Langley Research Center, Hampton, VA, 1996.

[101] J. Plancher, "Thermal and fluid design analysis of a square lattice honeycomb nuclear rocket engine," M.S. thesis, University of Florida, 2002.

[102] H. Schlichting, Boundary-layer theory, McGraw-Hill, New York, 1979. 


\section{$\underline{\text { PART II }}$}

\section{TESTING AND CAHARACTERIZATION OF MONOCARBIDES AND URANIUM TRI-CARBIDES}




\section{TABLE OF CONTENTS}

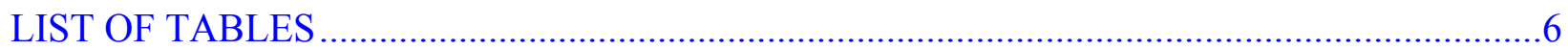

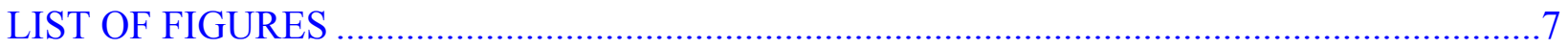

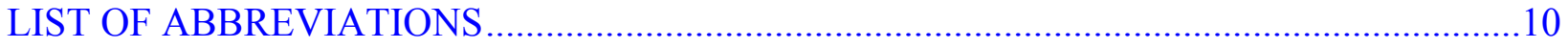

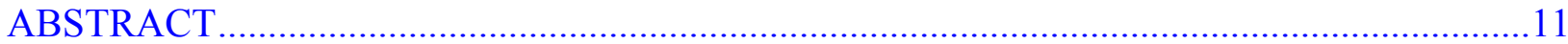

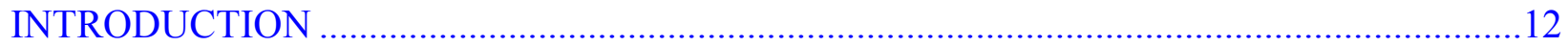

BACKGROUND AND LITERATURE REVIEW ............................................................... 14

2.1 Historical Relevance for Fuel Development.............................................................. 14

2.1.1 Pewee Series .................................................................................................. 15

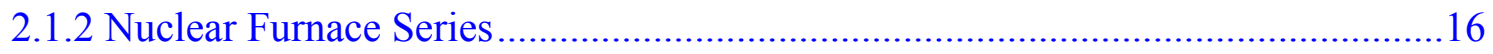

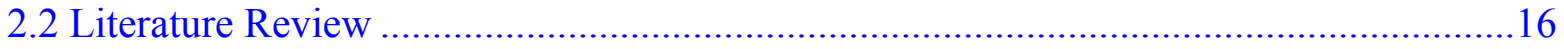

2.2.1 Hot Hydrogen Corrosion Mechanisms ................................................................16

2.2.2 Free Energy of Formation Calculations ..........................................................17

EXPERIMENT METHODS AND PROCEDURES …...................................................22

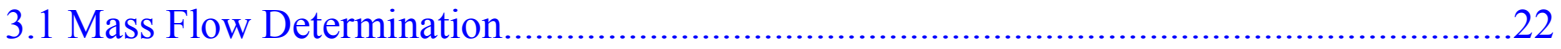

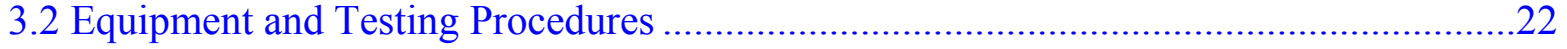

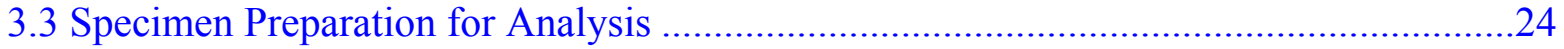

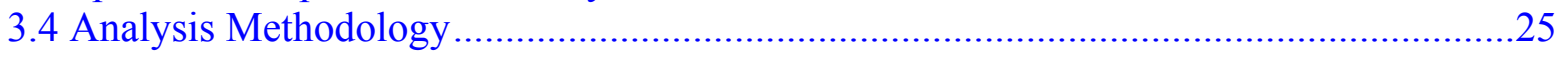

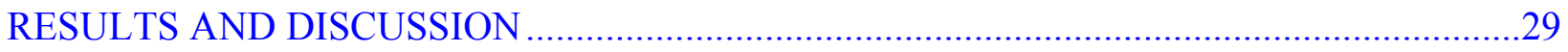

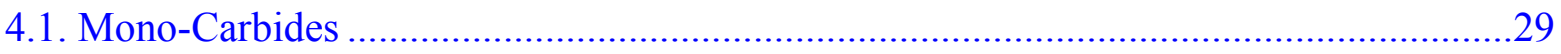

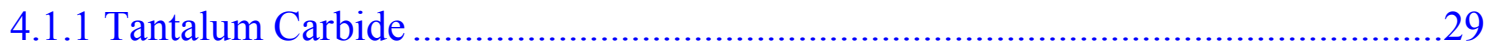

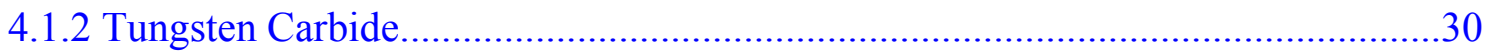

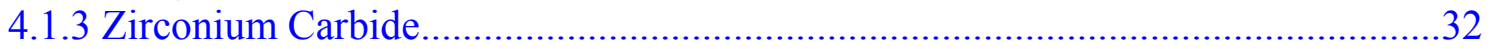

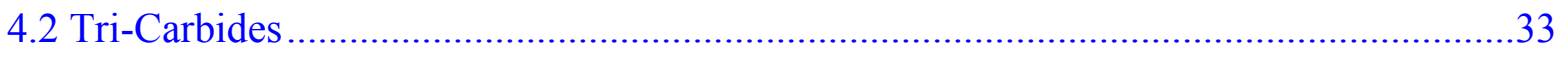

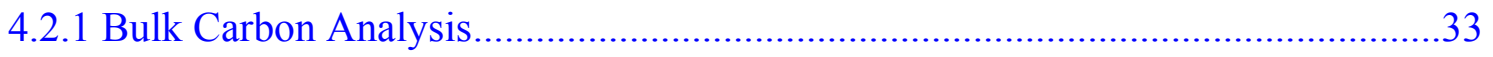

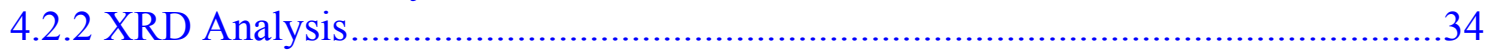

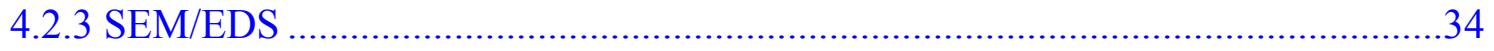

CONCLUSIONS AND RECOMMENDATIONS .........................................................63

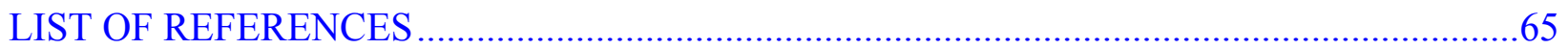




\section{LIST OF TABLES}

Table

page

Table 3-1. Average testing conditions for mono-carbide samples .........................................26

Table 3-2. Average testing conditions for tri-carbide samples .............................................26

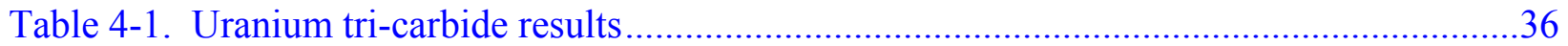




\section{LIST OF FIGURES}

$\underline{\text { Figure }}$

page

Figure 2-1. Four fuel forms used in Rover/NERVA designs (Matthews et al., 1991) .................19

Figure 2-2. Pseudo-binary Phase Diagram of $\left(\mathrm{U}_{0.1}, \mathrm{Zr}_{0.9}\right) \mathrm{C}_{\mathrm{x}}$ (Lyon, 1973) ..............................20

Figure 2-3. Standard free energy of formation of possible chemical reactions of monocarbides in a hydrogen environment.

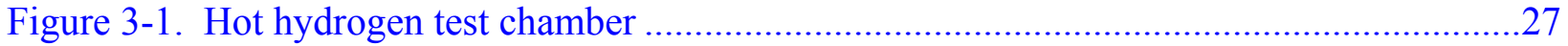

Figure 3-2. Top view of sample/tungsten susceptor/inductive coil configuration......................27

Figure 3-3. Copper cooled induction coil with tungsten susceptor assembly...........................28

Figure 4-1. EDS giving qualitative composition of TaC sample pre- and post-HHT.................37

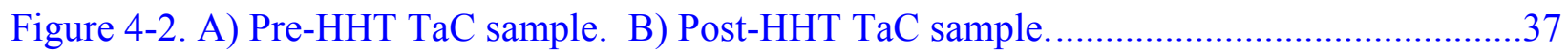

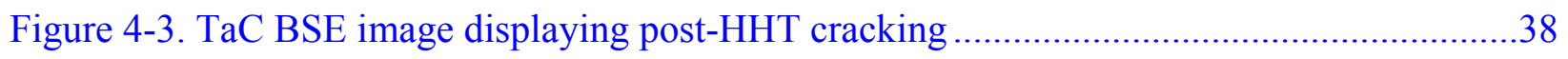

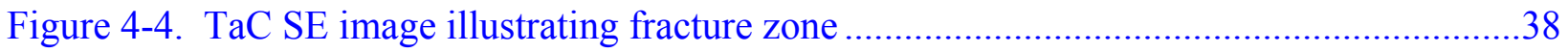

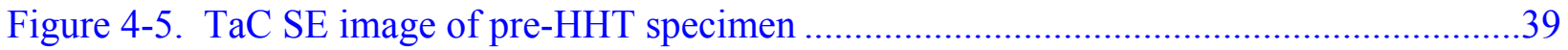

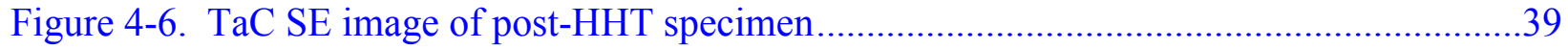

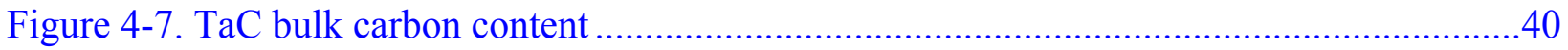

Figure 4-8. Mid-plane cross section showing TaC carbon content as a function of depth...........40

Figure 4-9. TaC pre- and post-HHT XRD spectrum ....................................................41

Figure 4-10. EDS giving qualitative composition of WC sample pre- and post-HHT................41

Figure 4-11. A) Pre-HHT WC sample. B) Post-HHT WC sample ...........................................42

Figure 4-12. WC SE image of pre-HHT specimen......................................................42

Figure 4-13. WC SE image of post-HHT specimen ....................................................43

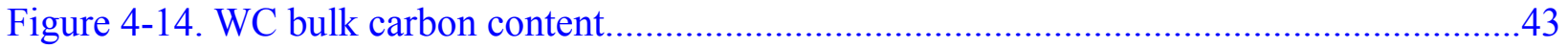

Figure 4-15. WC BSE image illustrating the transition zone where carbon depletion occurred...44

Figure 4-16. Mid-plane cross section shows WC carbon content as a function of depth. 44 
Figure 4-17. Mid-plane cross section of post-HHT showing WC transition zone of carbon content. .

Figure 4-18. WC pre and post-HHT XRD spectrum. ........................................................45

Figure 4-19. EDS giving qualitative composition of $\mathrm{ZrC}$ sample pre and post-HHT.................46

Figure 4-20. A) Pre-HHT ZrC sample. B) Post-HHT ZrC sample..........................................46

Figure 4-21. ZrC SE image of pre-HHT specimen........................................................4

Figure 4-22. ZrC SE image of post-HHT specimen ......................................................47

Figure 4-23. ZrC BSE image of pre-HHT specimen .....................................................48

Figure 4-24. ZrC BSE image of post-HHT specimen...........................................................48

Figure 4-25. ZrC bulk carbon content...........................................................................49

Figure 4-26. Mid-plane cross section shows $\mathrm{ZrC}$ carbon content as a function of depth.............49

Figure 4-27. ZrC pre- and post-HHT XRD spectrum....................................................50

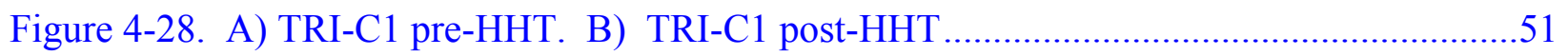

Figure 4-29. A) TRI-C2 pre-HHT. B) TRI-C2 post-HHT ...............................................51

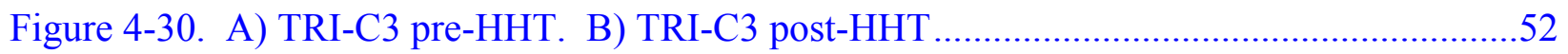

Figure 4-31. A) TRI-C4 pre-HHT. B) TRI-C4 post-HHT ..................................................52

Figure 4-32. TRI-C1 pre- and post-HHT bulk carbon content .............................................53

Figure 4-33. TRI-C2 pre- and post-HHT bulk carbon content ..............................................53

Figure 4-34. TRI-C3 pre- and post-HHT bulk carbon content .............................................54

Figure 4-35. TRI-C4 pre- and post-HHT bulk carbon content ...........................................54

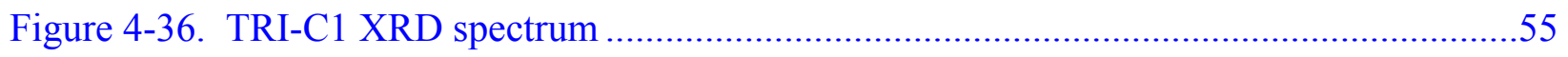

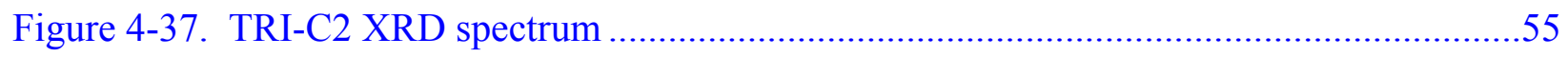

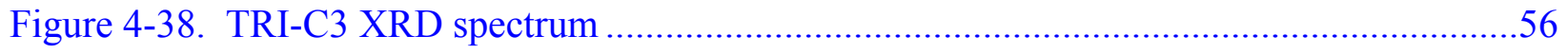

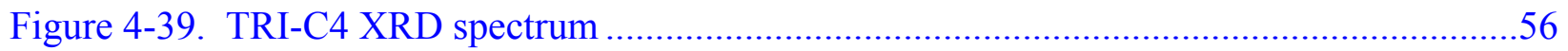

Figure 4-40. TRI-C1 BSE image of pre-HHT specimen ..................................................57

Figure 4-41. TRI-C1 SE image of post-HHT specimen ..................................................57 
Figure 4-42. TRI-C2 BSE image of pre-HHT specimen ................................................58

Figure 4-43. TRI-C2 BSE image of post-HHT specimen.................................................58

Figure 4-44. TRI-C3 BSE image of pre-HHT specimen ..................................................59

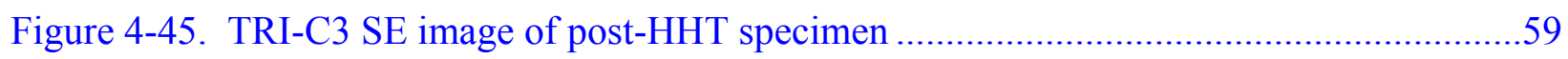

Figure 4-46. TRI-C4 SE image of pre-HHT specimen ..................................................6

Figure 4-47. TRI-C4 BSE image of post-HHT specimen..................................................60

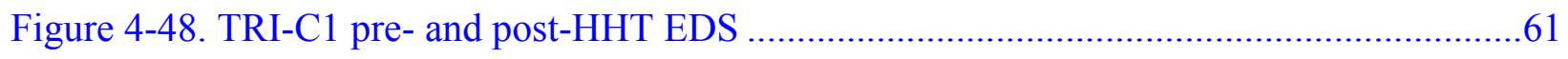

Figure 4-49. TRI-C2 pre-and post-HHT EDS. ...............................................................61

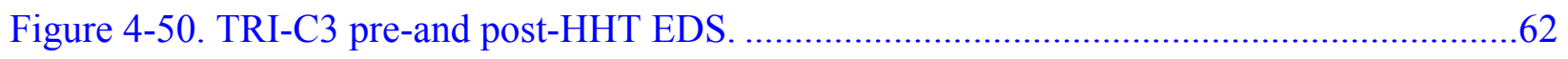

Figure 4-51. TRI-C4 pre-and post-HHT EDS .............................................................62 


\section{LIST OF ABBREVIATIONS}

$\begin{array}{ll}\text { BSE } & \text { BackScatter Electron } \\ \text { C-wt } \% & \text { Carbon Weight percent } \\ \text { EDS } & \text { Energy Dispersive Spectrum } \\ \text { EMPA } & \text { Electron MicroProbe Analysis } \\ \text { HHT } & \text { Hot Hydrogen Testing } \\ \text { HIP } & \text { Hot Isostatic Press } \\ \text { INSPI } & \text { Innovative Nuclear Space Power and Propulsion Institute } \\ \text { ISP } & \text { Specific Impulse } \\ \text { LANL } & \text { Los Alamos National Laboratory } \\ \text { MW } & \text { Megawatt thermal } \\ \text { NASA MSFC National Aeronautics and Space Administration Marshall Space Flight Center } \\ \text { NEP } & \text { Nuclear Electric Propulsion } \\ \text { NERVA } & \text { Nuclear Engine Rocket Vehicle Application } \\ \text { NF } & \text { Nuclear Furnace } \\ \text { ZrC } & \text { Zirconium Carbide } \\ \text { NTP } & \text { Nuclear Thermal Propulsion } \\ \text { PyC } & \text { Pyrolitic-Carbon } \\ \text { SE } & \text { Secondary Electron } \\ \text { SEM } & \text { Scanning Electron Microscopy } \\ \text { TaC } & \text { Tuntalum Carbide } \\ \text { X-Rsten Carbide }\end{array}$




\begin{abstract}
This project required the testing of three mono-carbides to investigate which carbide has the least degradation of microstructural properties. This was performed in order to determine the usability of various carbides in a base mixture. The purpose of this research was to investigate carbides as candidate fuel matrix for nuclear thermal propulsion. Tantalum carbide (TaC), tungsten carbide (WC), and zirconium carbide $(\mathrm{ZrC})$ were all tested in a hot hydrogen environment at an average temperature of $2775 \mathrm{~K}$. Each study tracked the carbon content, corrosion, density, hydrogen embrittlement, and phase changes. The results show that some carbides experienced changes in all of the aforementioned properties, while others experienced a combination of changes or no noticeable degradation.

The second stage of the research tested solid solution uranium bearing tri-carbides in a hot hydrogen environment. The tri-carbides included the compositions $\left(\mathrm{U}_{0.1}, \mathrm{Zr}_{0.58}, \mathrm{Nb}_{0.32}\right) \mathrm{C}_{0.95}$, $\left(\mathrm{U}_{0.1}, \mathrm{Zr}_{0.68}, \mathrm{Nb}_{0.22}\right) \mathrm{C}_{0.95},\left(\mathrm{U}_{0.1}, \mathrm{Zr}_{0.77}, \mathrm{Nb}_{0.13}\right) \mathrm{C}_{0.95}$, and $\left(\mathrm{U}_{0.05}, \mathrm{Zr}_{0.62}, \mathrm{Nb}_{0.33}\right) \mathrm{C}_{0.95}$. All tri-carbides were exposed to temperatures above $2900 \mathrm{~K}$ for two hours. Scanning electron microscopy, bulk carbon analysis, $\mathrm{x}$-ray diffraction, density measurements, and mass measurements were used to characterize each specimen Analysis showed that differences in metal composition had no noticeable effect on final fuel integrity. Carbon analysis, electron microscopy, and diffraction analysis showed that tri-carbides displayed little to no change in microstructure due to exposure to a hot hydrogen environment.
\end{abstract}




\section{CHAPTER 1}

\section{INTRODUCTION}

Fuel choice is a key element of nuclear reactors' use in space applications, particularly because of concerns about fuel performance. The nuclear thermal propulsion (NTP) and some nuclear electric propulsion (NEP) advanced space reactors must also consider the high temperature and high power density that the fuel experiences. Nuclear reactors intended for space applications will be required to achieve temperatures of $2500 \mathrm{~K}$ or more. For NTP the high temperatures ensures high specific impulse (ISP) and for NEP efficient heat rejection. High melting point carbides or refractory carbides are capable of surviving these conditions for an extended prototypic core lifetime making them a suitable fuel choice.

Refractory carbides have operating temperature estimated at $3000 \mathrm{~K}$. This high temperature capability makes them a preferred fuel choice, particularly when compared to other fuel candidates such as uranium dioxide, uranium carbide, or uranium nitride. The thermal conductivity of solid solution carbides approaches that of elemental uranium. This lowers the thermal gradient between the fuel and propellant, thus reducing thermal stress-induced cracking. The high thermal conductivity also allows for higher operating temperature with a smaller threat of fuel centerline melting. Carbides also exhibit low volatility, low density, and good neutronic properties such as good moderation due the presence of carbon and low absorption cross section.

A method for fabricating refractory carbides was established in previous research [1]. Solid solution uranium tri-carbide have been produced with the compositions $(\mathrm{U}, \mathrm{Zr}, \mathrm{Nb}) \mathrm{C}$, $(\mathrm{U}, \mathrm{Zr}, \mathrm{Ta}) \mathrm{C},(\mathrm{U}, \mathrm{Zr}, \mathrm{Hf}) \mathrm{C}$, and $(\mathrm{U}, \mathrm{Ta}, \mathrm{Nb}) \mathrm{C}$. This paper will address how microstructural integrity change by exposing tri-carbides to prototypic NTP/NEP temperatures. A hydrogen environment was examined because of its role in both types of systems: in NTP systems hydrogen is both the coolant and the propellant, while the NEP designs, such as the Pellet Bed Reactor concept or 
Neptune reactor concept, it acts as a coolant. Uranium tri-carbide was chosen for testing because preliminary results showed that tri-carbides have higher melting temperatures and better resistance to hot hydrogen corrosion than the binary-carbide fuel (U,Zr)C [2].

Testing materials in a high temperature hydrogen environment is rare because most operating conditions do not warrant its use. As a result, background information and literature is limited. The main concern with NTP is hot hydrogen corrosion. Hydrogen at high temperatures can erode carbon from the matrix or react and corrode the fuel substrate to form methane or other hydrocarbons. Studies of previous NTP programs and case studies have shown that the hot hydrogen corrosion mechanisms are a highly coupled and highly complex process. Research shows that carbide's material properties are dependent on maintaining stoichiometry [3]. Changes in the fuel's carbon-to-metal ratio can lead to changes in the liquidus, changes in phase orientation, fatigue resistance, and toughness. A quantification of carbon-to-metal ratio with exposure time to temperature is needed to ensure properties such as melting point, thermal conductivity, and consistent thermal expansion are coherent throughout the fuel's anticipated lifetime. Evidence of hot hydrogen corrosion can show as hydrogen induced blistering, hot embrittlement, mass loss, and hydride formation. 


\section{CHAPTER 2}

\section{BACKGROUND AND LITERATURE REVIEW}

\subsection{Historical Relevance for Fuel Development}

Nuclear reactors for rocket applications were first ground tested during the Rover program. The main purpose of the Rover program was to develop nuclear powered rockets for ballistic missile defense. When the need for nuclear rocket applications diminished due to the efficiency of chemical rockets, Los Alamos National Laboratory (LANL) contracting with Westinghouse Electric Corporation created a research and development program within the original framework of the Rover program. The new program, the Nuclear Engine for Rocket Vehicle Applications (NERVA), was developed for space purposes. The NERVA program was charged not only with reactor design, but also to achieve the highest possible propellant temperature. The intent was a completed rocket design with maximized ISP while maintaining low weight.

Equation 1 defines ISP as proportional to the square root of the fluid temperature over the fluid molecular weight [2]. Hydrogen is the de facto coolant for NTP because its molecular weight is the lowest and highest specific heat of all elements. All parameters being equal, the temperature of the coolant dictates the efficiency of the rocket design. Therefore, fuel development was rigorously pursued in order to meet this goal. Throughout the course of the Rover program several reactors series with varying fuel forms were investigated. Figure 2-1 illustrates the fuels used during the Rover/NERVA program. Nuclear reactors cores developed during these programs generally operated with temperatures around $2500 \mathrm{~K}$ and pressures of 3MPa. The series that is most important for this research, the Pewee and Nuclear Furnace families, focused on alternative fuels, higher fuel temperature, and fuel performance. 


$$
E S P-A C_{f} \sqrt{\frac{T_{g h}}{M_{\theta}}}-\frac{F}{m}
$$

Eqn. 1

$A=$ performance factor related to thermo-physical properties of the propellant

$\mathrm{C}_{\mathrm{f}}=$ thrust coefficient, which is a function of nozzle parameters

$\mathrm{T}_{\mathrm{ch}}=$ chamber temperature $(\mathrm{K})$

$\mathrm{M}_{\mathrm{c}}=$ molecular weight of propellant

$\mathrm{F}=$ thrust $(\mathrm{N})$

$\mathrm{m}=$ mass flow rate of propellant $(\mathrm{kg} / \mathrm{s})$

\subsubsection{Pewee Series}

The Pewee series was intended to be a family of small inexpensive test reactors used for high temperature fuels demonstration: only one reactor was eventually built and demonstrated. In 1968 the Pewee 1 ran successfully at $503 \mathrm{MW}_{\text {th }}$ for 40 minutes with an exit coolant temperature of $2550 \mathrm{~K}$. The peak fuel temperature was measured at $2750 \mathrm{~K}$. It demonstrated the highest power for a reactor of its size, with an average power density of $2340^{M \mathrm{~W}} / \mathrm{m}^{2}$ and a peak power density of $5 \mathbf{5 2 0 0} M W W_{m^{\mathrm{g}}}$. The calculated ISP in vacuum was $845 \mathrm{~s}$. Pewee contained 402 hexagonal fuel elements loaded with pyrolytic-graphite coated $\mathrm{UC}_{2}$ particles dispersed throughout a graphite matrix. The fuel elements were coated with $\mathrm{NbC}, \mathrm{ZrC}$, and $\mathrm{Mb}$ as an overcoat. Inspection of the fuel elements after testing showed that many of the elements exhibited cracked regions or external corrosion. Fractures were due to thermal-mechanical stresses, but corrosion was attributed to the fuel elements' graphite matrix being subjected to a hot hydrogen environment [4]. 


\subsubsection{Nuclear Furnace Series}

The Nuclear Furnace (NF) series had a purpose similar to the Pewee series. Like the Pewee 1, only one reactor in this series was built and operated. This was due to changes in national priorities, which forced the Rover/NERVA program to end in 1973. It was the last reactor built and tested during the Rover program. The NF 1 served as the test bed for two new types of fuel forms: (U,Zr)C composite and (U,Zr)C solid solution binary carbide. This was a $44 \mathrm{MW}_{\text {th }}$ reactor that operated for 109 minutes with an average exit coolant temperature of 2450K. Unlike Pewee 1, this reactor contained 49 hexagonal fuel elements. For this test, the solid solution binary carbide fuel elements had no protective coating on the surface and showed minimal corrosion compared to the other fuel forms. It did, however, show extensive cracking. The composite performed better than the solid solution binary carbide in crack resistance but corrosion from hydrogen contacting the graphite matrix continued to be a problem [5].

\subsection{Literature Review}

\subsubsection{Hot Hydrogen Corrosion Mechanisms}

A review of past studies has shown that hot hydrogen corrosion is a highly complex and highly coupled process. That study showed that the corrosion process is a combination of the following: (1) exposure of the fuel to hot hydrogen gas, (2) thermal and mechanical cycling and loading, (3) radiation induced damage to the fuel matrix, and (4) annealing effect from high temperature creep [6]. The first process is responsible for the corrosion of the microstructure. The last three mechanisms influenced the rate at which the fuel matrix was degraded.

Hydrogen is able to penetrate the microstructure through micro-cracks and surface pores. Depending on the local conditions such as local temperature, pressure, and composition a free energy change or chemical reaction can occur and give rise to hydrocarbon reaction products. Typically reaction products occupy a greater volume than the reactants. This causes stress- 
corrosion cracking, which consequently creates a larger surface area and deeper route for the hot hydrogen to attack. The corrosion-induced cracks and pores weaken the microstructure and allow for surface erosion. The depletion of carbon from the fuel surface leads to changes in local composition which alters local material properties. In solid solution uranium bi-carbides it has been shown that material properties depend heavily on the carbon-to-metal ratio. Figure 2-2 shows a phase diagram of a pseudo-binary carbide $\left(\mathrm{U}_{0.1}, \mathrm{Zr}_{0.9}\right) \mathrm{C}_{\mathrm{x}}$. In this composition the carbon-to-metal ratio is limited to $0.92-0.96$ before a solid fuel transforms to a mixture of liquid/solid solution carbide. Allowing this to occur will ultimately lead to fuel failure.

Fuel irradiation enhances the rate of hot hydrogen corrosion. Exposure of the microstructure to radiation, particularly fast neutrons, creates lattice dislocations and point defects. The localized defect effectively reduces the local thermal conductivity which produces a thermal gradient with the surrounding lattice sites. The defects also reduce local ductility. This, combined with the reduction in thermal conductivity produces thermal stress-induced cracking that exposes more surface area to hot hydrogen attack.

Creep is a mechanism that does not enhance the hot hydrogen corrosion rate, unlike the other two processes. The operating temperatures of NTP fuel accelerate the creep process. Creep will serve to anneal the localized high stress zones deterring the formation of crack growth and closing micro-cracks within the microstructure.

\subsubsection{Free Energy of Formation Calculations}

Binary carbides and ternary carbides are materials whose full use has yet to be investigated. As a result, there is no tabulated data on the free energy of formation to determine the system's thermodynamic stability. The free energy of formation for the mono-carbides was calculated to determine the system's stability in a hydrogen environment. An approximation of 
ternary carbide system stability was made by observing the Gibbs potential for mono-carbides since uranium tri-carbide is a solid solution of $(\mathrm{U}, \mathrm{Zr}, \mathrm{X}) \mathrm{C}$ where $\mathrm{X}$ is a metal.

The second law of thermodynamics was used to determine whether the mechanism for microstructural damage to mono-carbides was predominantly corrosion or erosion. The Standard Free Energy of Formation $\left(\Delta \mathrm{G}_{\mathrm{f}}{ }^{\circ}\right)$ gives the work released or consumed by a chemical reaction at constant temperature and pressure. If $\Delta \mathrm{G}_{\mathrm{f}}{ }^{\circ}$ is positive the chemical reaction is non-spontaneous and if $\Delta \mathrm{G}_{\mathrm{f}}{ }^{\circ}$ is negative the chemical reaction is spontaneous. A positive $\Delta \mathrm{G}_{\mathrm{f}}{ }^{\circ}$ means that the species reaction cannot take place under the given conditions without the minimum of the $\Delta \mathrm{G}_{\mathrm{f}}^{\circ}$ calculated [7]. The $\Delta \mathrm{G}_{\mathrm{f}}{ }^{\circ}$ was calculated for several chemical reactions that are possible in a hydrogen environment, neglecting the presence of trace elements such as oxygen in the testing system. The dissociation energy of diatomic hydrogen is $4.5 \mathrm{eV}$. This is higher than the thermal energy of the system, $2700 \mathrm{~K}$ or $0.232 \mathrm{eV}$. For simplification, only atomic hydrogen would be considered because of its chemical volatility. Figure 2-3 displays the Gibbs free energy plot for several reactions. With the exception of one chemical reaction each of the other reactions are not possible at the temperatures of interest without the addition of more work. This implies that the main mechanism for microstructural damage is erosion rather than corrosion. The one chemical reaction that is spontaneous is $\mathrm{WC}+4 \mathrm{H} \rightarrow \mathrm{W}+\mathrm{CH}_{4}$ which readily occur at temperatures beneath $445 \mathrm{~K}$ or its equilibrium temperature. This is a reaction that is plausible for corrosion to occur and carbon loss to be significant. Using this principle the mono-carbide, WC, should experience a noticeable loss in carbon content. Since erosion is the mechanism for damaging the TaC and $\mathrm{ZrC}$ matrix those compounds could exhibit a uniform loss of both metal and carbon. 


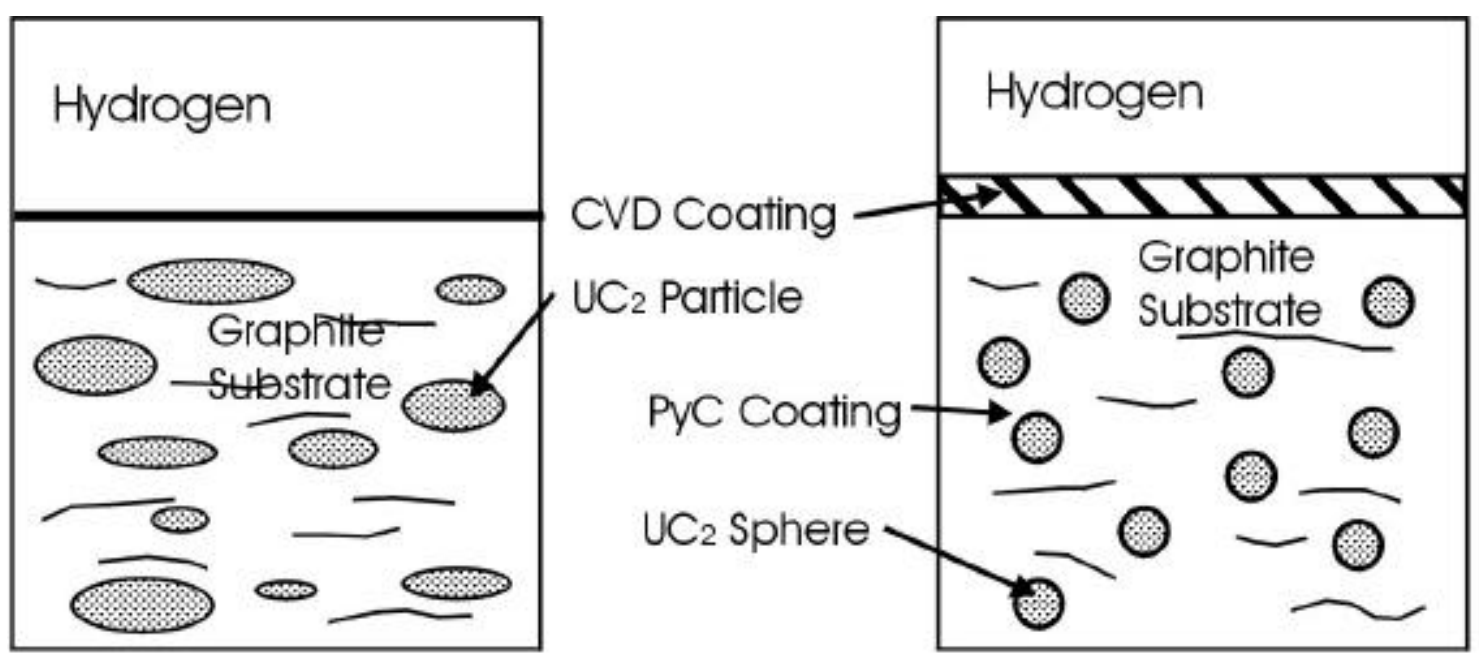

a) $\mathrm{UC}_{2}$ Particles Dispersed

b) PyC Coated UC 2 Spheres in Graphite Matrix

Dispersed in Graphite

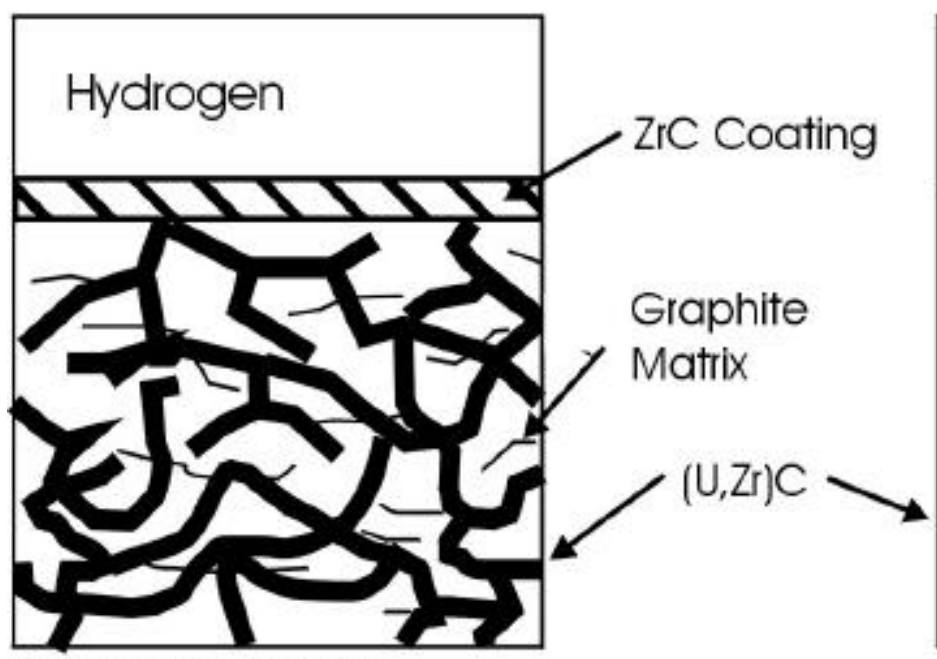

c) Composite Fuel

Hydrogen

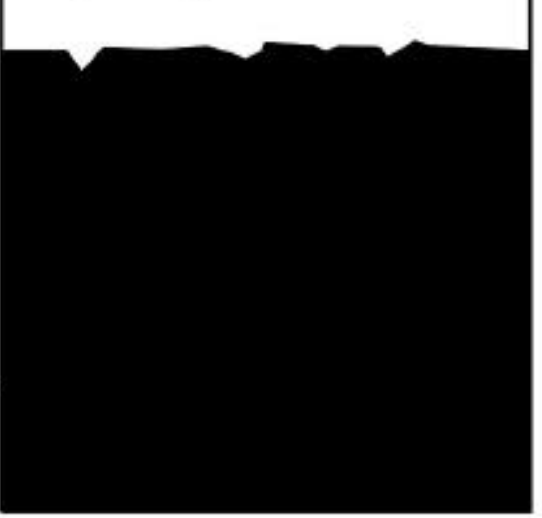

d) Solid Solution $(U, \mathrm{Zr}) \mathrm{C}$

Figure 2-1. Four fuel forms used in Rover/NERVA designs (Matthews et al., 1991) 


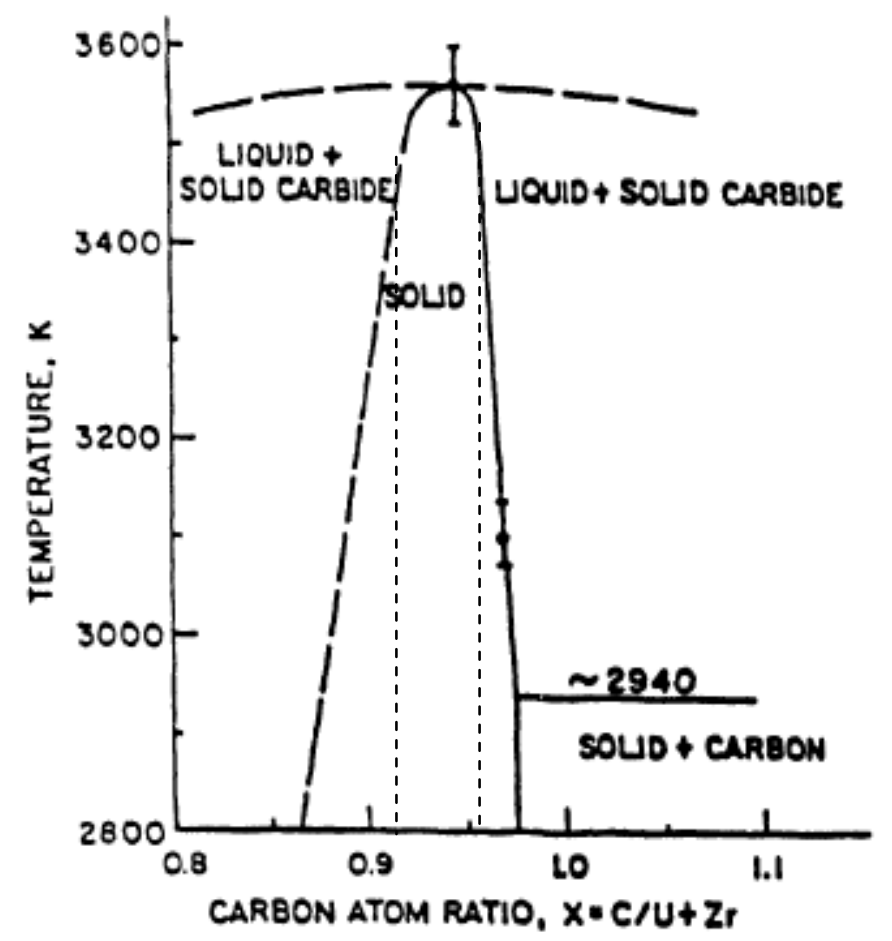

Figure 2-2. Pseudo-binary phase diagram of $\left(\mathrm{U}_{0.1}, \mathrm{Zr}_{0.9}\right) \mathrm{C}_{\mathrm{x}}$ (Lyon, 1973) 


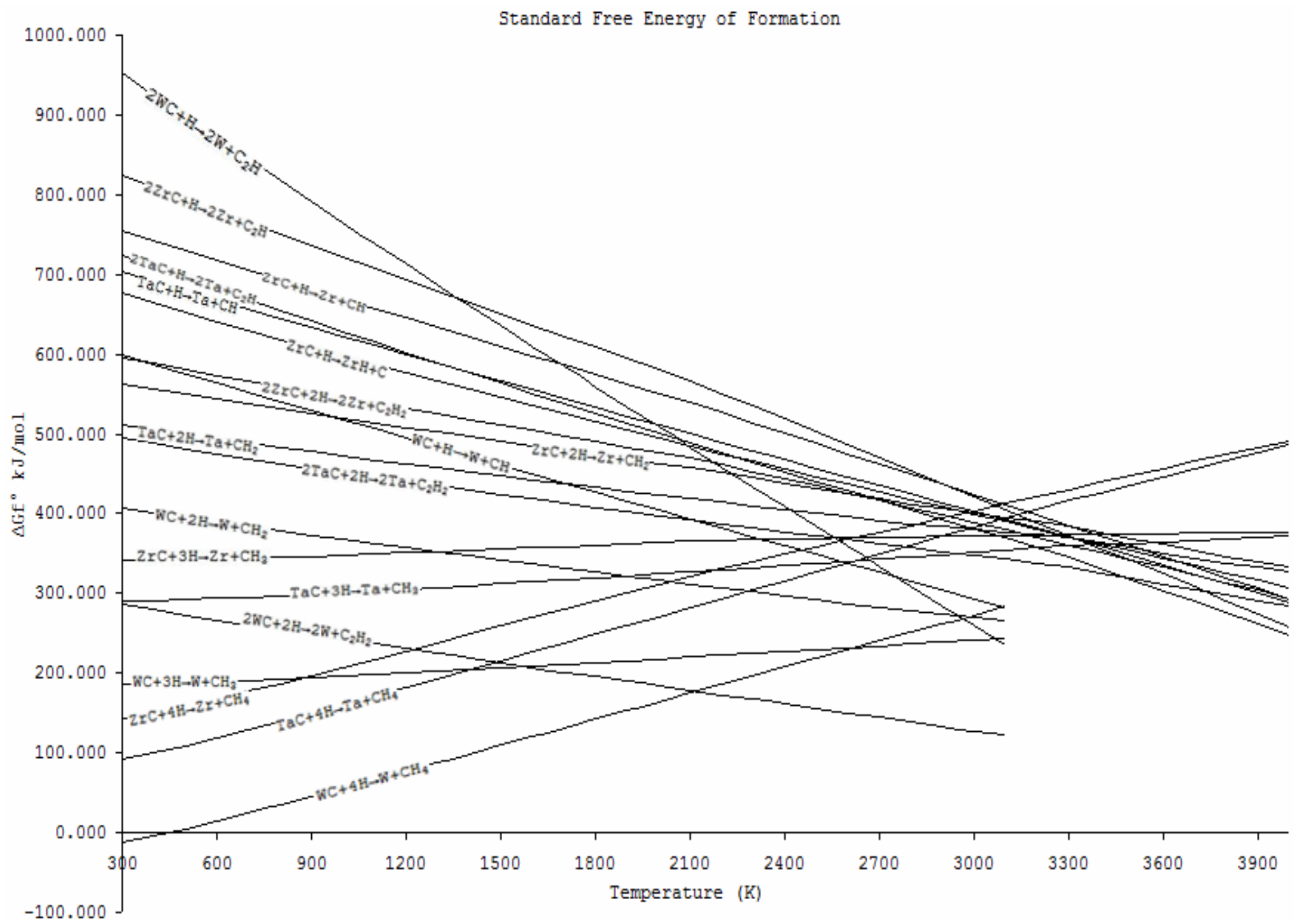

Figure 2-3. Standard free energy of formation of possible chemical reactions of mono-carbides in a hydrogen environment $[8,9,10]$ 


\section{CHAPTER 3}

\section{EXPERIMENT METHODS AND PROCEDURES}

\subsection{Mass Flow Determination}

A linear relationship was used to ensure that the samples tested in this experiment experienced a relative mass flow rate similar to a nuclear space reactor. The Pewee reactor from the Rover/NERVA program was used as a template. The Pewee reactor used a total hydrogen flow rate of $19.341^{\mathrm{kg}} / \mathrm{s}$ with $14.62^{\mathrm{kg} / \mathrm{s}}$ cooling the fuel. The total available coolant area of was an $858,929 \mathrm{~cm}^{2}$ based on dimensions of the core [4]. This gives a mass flow per square

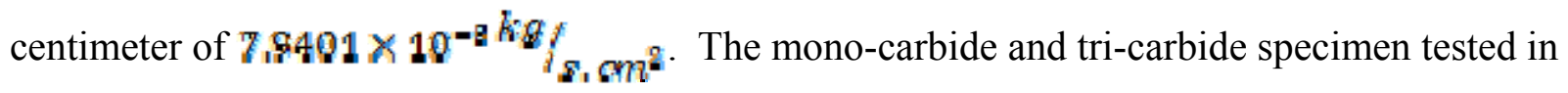
this experiment had an available coolant area of approximately $3.5 \mathrm{~cm}^{2}$ resulting in an equivalent mass flow rate of $2.71 \times 10^{-4} \mathrm{~kg} / \mathrm{s}$. The dimensions of each specimen vary slightly making $3.5 \mathrm{~cm}^{2}$ the estimated sample surface the coolant contacts. Chamber pressure, temperature, and mass flow rate were recorded every 10 minutes to calculate and average testing conditions. All average testing conditions are listed in tables 3-1 and 3-2.

\subsection{Equipment and Testing Procedures}

Two series of samples were tested. Mono-carbide samples produced at National Aeronautics and Space Administration's Marshall Space Flight Center (NASA MSFC) and uranium tri-carbides produced at University of Florida's Innovative Nuclear Space Power and Propulsion Institute (INSPI). The mono-carbides were fabricated by hot Isostatic press (HIP). Tri-carbides were fabricated by an induction heated uni-axial hot press liquid phase sintering method [1]. During this process a homogeneous mixture of three carbides are cold pressed and heated to a temperature above the lowest melting point carbide constituent, in this case uranium 
carbide. The liquid uranium carbide fills in porous areas and contacts the grain boundary from other the carbide component, thereby quickening the diffusion and resulting in a solid solution tri-carbide fuel.

Figure 3-1 illustrates the hot hydrogen test equipment utilized to create the desired conditions. Samples were placed in a chamber that is evacuated to a maximum 100millitorr then backfilled with hydrogen in order to purge the system of oxygen and other gas impurities. This was performed three times to minimize oxidation of the sample and chamber components. The system was then purged and filled with hydrogen to the positive pressure, with respect to atmospheric pressure, to ensure air could not enter the chamber. The pressure was monitored with an Omega pressure transducer. The experiment was powered by a LEPEL Solid State RF generator. Ultra pure hydrogen then flowed from a gas cylinder through the system for a prototypic NTP run time. Hydrogen flow rate was measured by an OMEGA FMA-873 mass flow controller. The temperature was monitored through the use of a MAXLINE IRCON active control and measured by a MAXLINE IRCON two color infrared pyrometer. The pyrometer was aligned with a viewport on the chamber to permit observations to be made. The sample was surrounded by a tungsten susceptor that was inductively heated by a water cooled copper coil surrounding the tungsten susceptor. The sample was heated via radiative heat transfer from the tungsten susceptor. Figure 3-2 shows the sample-coil assembly housed in the vacuum chamber. Figure 3-3 shows the coil assembly with the hole drilled through the tungsten susceptor to allow the pyrometer to measure the inner surface wall of the tungsten susceptor. This provided a more accurate representation of the specimen temperature. A parametric temperature study, up to 2573K, was made with a C-type thermocouple to ensure accuracy of pyrometer readings. 


\subsection{Specimen Preparation for Analysis}

After testing was complete the samples were prepared for microstructural characterization. First, the test samples were sectioned with a LECO ${ }^{\circ}$ VC-50 diamond saw. A diamond blade was used to account for the hardness of carbides. Individual sections were taken for X-ray Diffraction (XRD), bulk carbon analysis, Electron MicroProbe Analysis (EMPA), and Scanning Electron Microscope (SEM) analysis. All specimens were ultrasonically cleaned and degassed in distilled water for 60 minutes with a Bransonic $\mathbb{R}$ B3-R. All specimens were dried in a desiccator for a minimum of 24 hours before the analysis was conducted.

\section{a. XRD Preparation}

i. Specimens were either pulverized to a powder in an alumina crucible or sectioned to approximately $0.5 \mathrm{~mm}$ thickness and mounted on glass slides.

\section{b. Bulk Carbon Analysis}

i. Specimens prepared for bulk carbon analysis were pulverized to a powder in an alumina crucible.

ii. Three to five batches weighing $0.25 \mathrm{~g}$ from each sample composition were placed in a zirconia crucible. High purity accelerator was added to ensure samples could be inductively heated and ignited. A mixture of $1.8 \mathrm{~g}$ of copper and $1.2 \mathrm{~g}$ of iron was the amounts recommended by the equipment's operations manual. The iron carbon impurity content was 8PPM.

iii. Samples were placed in carbon analyzer for combustion analysis. The carbon determinator was calibrated using a tungsten carbide standard with $6.18 w t \%$ C.

c. SEM and EMPA Preparation 
i. Sections for SEM and EMPA were mounted in phenol ring molds using a 1:5 ratio LECO hardener to LECO epoxy resin, respectively.

ii. LECO ${ }^{\circledR}$ Spectrum System 1000 with semi-automatic heads was used for rough polishing mounted specimens from 180grit to 1200 grit.

iii. The final polishing steps decreased surface roughness from $15 \mu \mathrm{m}$ to $0.5 \mu \mathrm{m}$. Polishing was performed by hand.

iv. Sections intended for SEM were acid etched for one minute with nitric acid at $90 \%$ concentration.

v. Sections intended for EMPA was not acid etched.

\subsection{Analysis Methodology}

The following variables were examined for pre- and post-hot hydrogen testing (HHT): overall carbon concentration, carbon and metal concentration as a function of depth, density, and phase and crystallographic changes. Several visual observations such as grain growth, inter and intra-granular corrosion or erosion, spallation, cracking, swelling, and hydrogen embrittlement were also made. The pre- and post-HHT density was measured by immersion testing. A LECO WC-200 carbon determinator was used to combust specimens to investigate the bulk carbon concentration. A carbon profile of each specimen as a function of depth was characterized by EMPA. The EMPA instrument used was a JEOL SUPERPROBE 733 Electron Probe.

Topographical and compositional contrast and elemental composition were inspected with a JEOL JSM-6400 SEM. SEM was conducted to make most visual observations. Using XRD phase and crystallographic properties were analyzed with Philips APD 3720 X-Ray Diffractometer and reference spectra database [11]. Specimen weight and dimensions were measured with the Sartorius R180D analytic balance with an accuracy of $0.00001 \mathrm{~g}$ and a Mitutoyo Absolute Digimatic Caliper (CD-6" CSX), respectively. 
Table 3-1. Average testing conditions for mono-carbide samples

\begin{tabular}{c|c|c|c|c}
\hline & $\mathrm{TaC}$ & $\mathrm{WC}$ & $\mathrm{ZrC}$ & \\
\hline Mass Flow Rate & 15.58 & 15.19 & 15.74 & $g_{\text {min }}$ \\
\hline Temperature & 2762 & 2779 & 2776 & Kelvin \\
\hline Chamber Pressure & 968 & 969 & 970 & torr \\
\hline Run Time & 2 & 2 & $1.167^{*}$ & $\mathrm{hr}$ \\
\hline
\end{tabular}

*The $\mathrm{ZrC}$ sample test was conducted for a shorter time because of equipment constraints.

Table 3-2. Average testing conditions for tri-carbide samples

\begin{tabular}{|c|c|c|c|c|c|}
\hline & $\left(\mathrm{U}_{0.1}, \mathrm{Zr}_{0.58}, \mathrm{Nb}_{0.32}\right) \mathrm{C}_{0.95}$ & $\left(\mathrm{U}_{0.1}, \mathrm{Zr}_{0.68}, \mathrm{Nb}_{0.22}\right) \mathrm{C}_{0.95}$ & $\left(\mathrm{U}_{0.1}, \mathrm{Zr}_{0.77}, \mathrm{Nb}_{0.13}\right) \mathrm{C}_{0.95}$ & $\left(\mathrm{U}_{0.05}, \mathrm{Zr}_{0.62}, \mathrm{Nb}_{0.33}\right) \mathrm{C}_{0.95}$ & \\
\hline Mass Flow Rate & 15.81 & 14.92 & 14.92 & 14.92 & $\mathscr{P}_{\text {min }}$ \\
\hline Temperature & 2926 & 2903 & 2903 & 2903 & kelvin \\
\hline Chamber Pressure & 982 & 964 & 964 & 964 & torr \\
\hline Run Time & 2 & 2 & 2 & 2 & $\mathrm{hr}$ \\
\hline
\end{tabular}




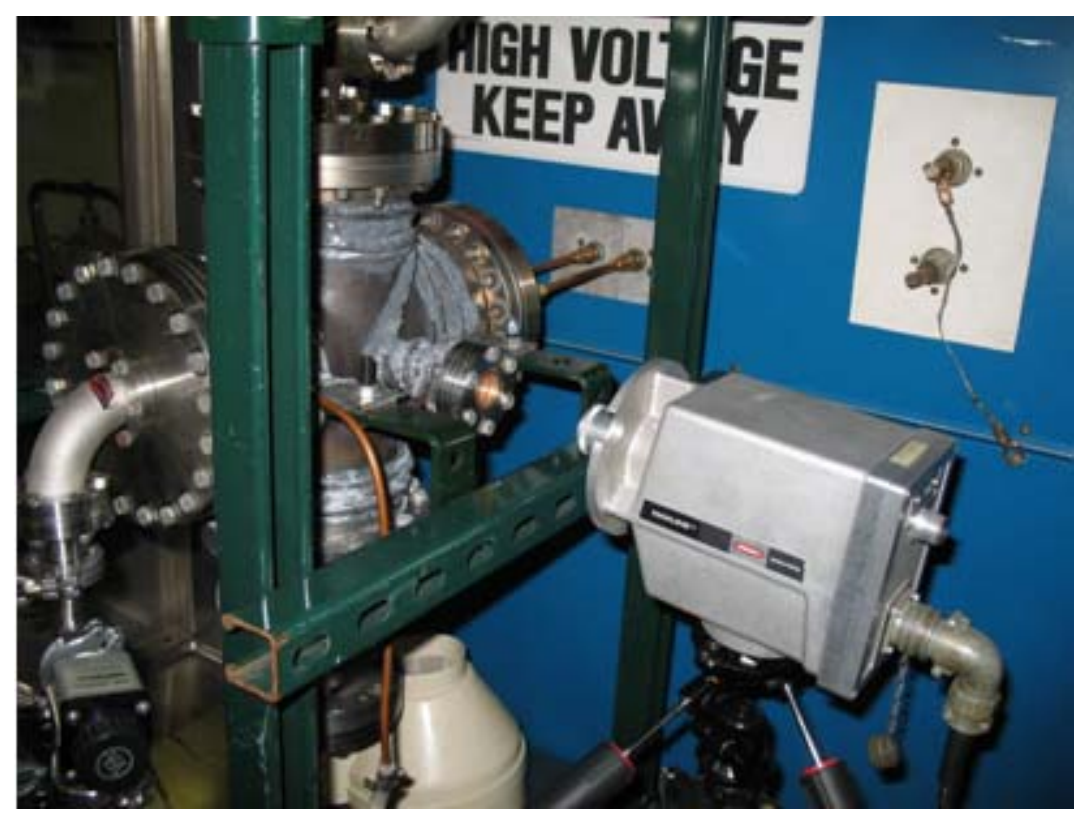

Figure 3-1. Hot hydrogen test chamber

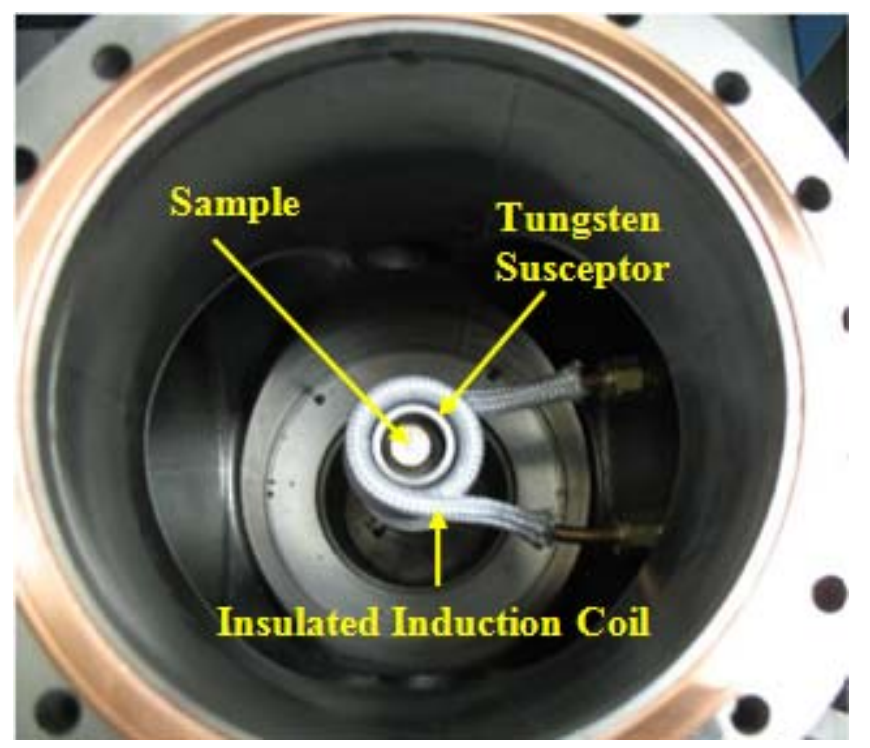

Figure 3-2. Top view of sample/tungsten susceptor/inductive coil configuration 


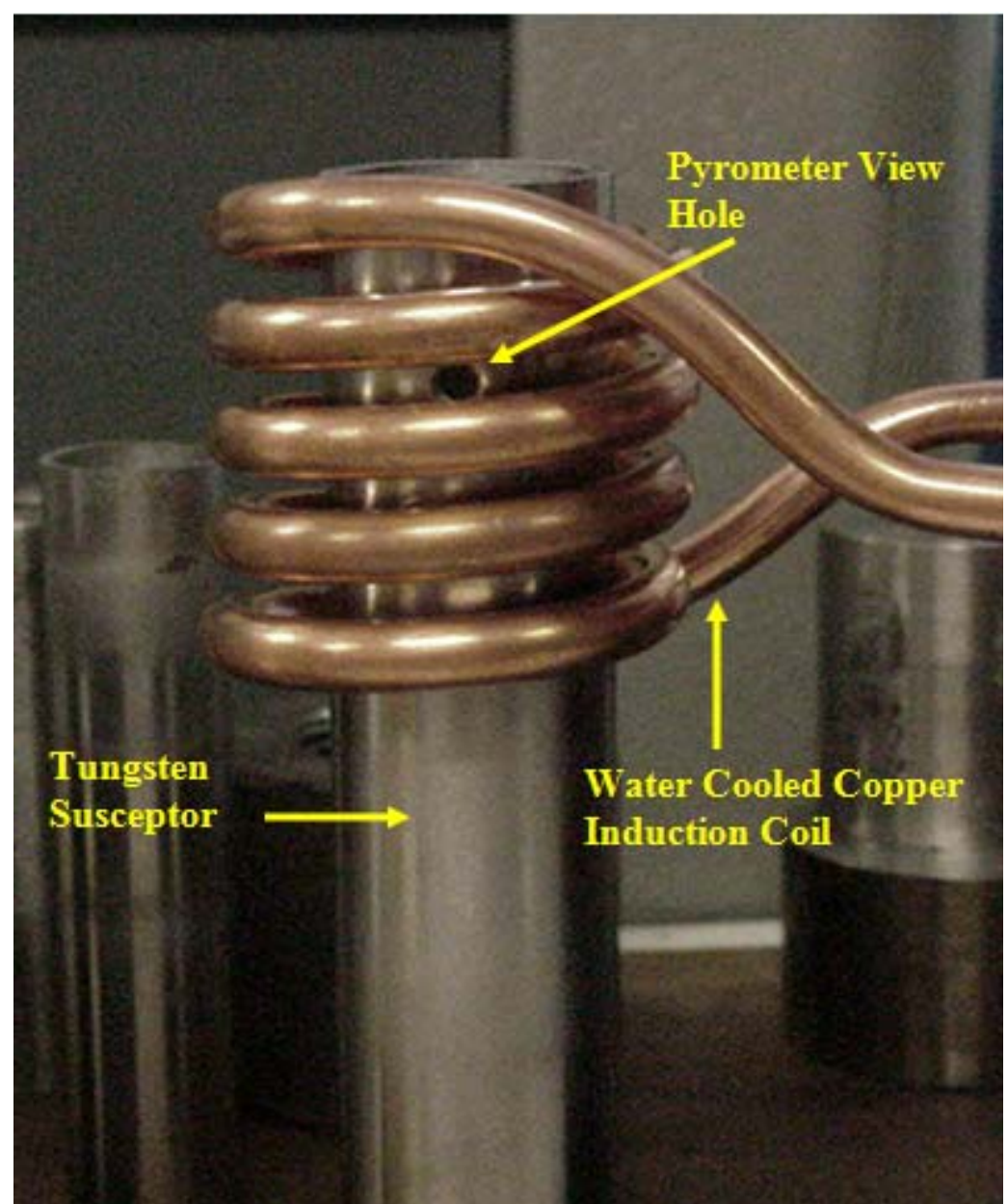

Figure 3-3. Copper cooled induction coil with tungsten susceptor assembly 


\section{CHAPTER 4}

\section{RESULTS AND DISCUSSION}

\subsection{Mono-Carbides}

\subsubsection{Tantalum Carbide}

To begin the analysis an energy dispersive spectrum (EDS) was taken to verify that the sample contains the given elements. Figure 4-1 shows the EDS both pre- and post-HHT. The post-HHT spectrum was taken to verify there was no contamination—atomic numbers higher than carbon-were introduced to the sample after HHT. Figure 4-2 gives a view of the pre- and post-HHT TaC samples. Compared to the pre-HHT sample, the post-HHT shows extensive cracking on the surface. A cross section was taken through the post sample and subsurface cracking was also present. Figure 4-3 and figure 4-4 shows the extent of cracking through a midaxial cross-section. Figures 4-5 and 4-6 show that the grain structure was unaffected by the presence of hydrogen. Neither grain growth nor grain degradation occurred, but the grain area adjacent to the cracks was fractured and porous.

$\mathrm{TaC}$ has a theoretical carbon weight percent $(\mathrm{C}-\mathrm{wt} \%)$ of $6.224 \mathrm{wt} \%$.. Figure 4-7 shows the pre-HHT C-wt $\%$ at $8.136 \mathrm{wt} \%$ and post-HHT at $7.975 \mathrm{wt} \%$, both which are hyper-stoichiometric. Statistically there was no change in carbon content. To determine if that small difference in the mean $\mathrm{C}-\mathrm{wt} \%$ for the pre- and post-HHT samples is due to the depletion of carbon at the surface from hydrogen flow, an EMPA was performed. The results of the EMPA shown in figure 4-8 illustrates that the pre- and post-HHT samples show no change in carbon concentration from the surface of the sample to within $360 \mu \mathrm{m}$. The data point at $400 \mu \mathrm{m}$ represents the center of the sample or $3 \mathrm{~mm}$ from the edge. The fluctuation in the EMPA profile is caused by surface roughness. 
The immersion density test showed that for the TaC pre-HHT sample the density was $14.350 \mathrm{~g} / \mathrm{cm}^{3}$. The post-HHT sample had $13.656 \mathrm{~g} / \mathrm{cm}^{3}$ yielding a percent change of $4.836 \%$.

The reference theoretical density for $\mathrm{TaC}$ is $14.498 \mathrm{~g} / \mathrm{cm}^{3}$ making the pre-HHT sample $98.98 \%$ dense and the post-HHT sample $94.19 \%$ dense after a two hour run time. Since pores are often the sites for crack initiation it is possible that pores are the cause of the extensive cracking throughout the $\mathrm{TaC}$ sample.

The XRD analysis for the pre- and post-HHT showed that the structure did not undergo a phase change. Figure 4-9 displays the results. If any of the reactions in figure 2-3 would have occurred, peaks indicating elemental tantalum would have been present.

\subsubsection{Tungsten Carbide}

The EDS displayed in figure 4-10 indicates that the elemental compositions for the preand post-HHT samples are consistent. The other analysis for the post-HHT sample shows a large difference in properties from the pre-HHT. The blistering on the surface in figure 4-11b indicates the formation of a liquid or a gas. Assuming there were trace amounts of oxygen in the system and the formation of tungsten oxide was negligible, the blistering on the surface could be attributed to trapped methane gas. The post-HHT WC sample shows that the degradation occurred at the grain boundaries. An explanation for the blistering is the mobility of the atomic hydrogen in the grain boundaries bonding with carbon at the boundaries to form methane. That methane then diffuses though the boundaries to the surface. The gas that did not escape was trapped under the surface to blister. Significant grain growth occurred in the WC sample. Figure 4-12 and figure 4-13 show the pre-HHT and post-HHT grain structure respectively. this can be attributed to conducting the testing near the melting point of tungsten carbide $(3143 \mathrm{~K})$. 
Figure 4-14 indicates that the bulk carbon content for the pre-HHT sample was $12.7 \%$ while the post-HHT sample had a C-wt $\%$ of 5.928 . Since pre-and post-HHT carbon content are outside of their statistical error then depletion of carbon from the microstructure occurred with certainty. The large difference in the pre- and post-HHT carbon content was due to carbon depletion at the surface of the WC in the post-HHT sample. Figure 4-15 shows a BSE image giving compositional contrast of the WC post-HHT. The brighter edge region indicates there was higher tungsten content at the edge. The depletion of carbon at the edge was verified using EMPA. Figure 4-16 shows that the post-HHT sample has less carbon content within the first $360 \mu \mathrm{m}$ from the edge. The data point at $400 \mu \mathrm{m}$ represents the center or $3 \mathrm{~mm}$ from the edge of the sample. That data point at the center of the sample indicates there is a diffusion gradient of carbon in the post-HHT sample. Figure 4-17 illustrates that at a depth of $630 \mu \mathrm{m}$, the WC postHHT sample's depleted carbon zone returns to the carbon concentration of the pre-HHT sample. This gives a carbon depletion depth rate approximately $5.25^{\mu \mathrm{m}} / \mathrm{min}$.

Notice the difference between the pre-HHT bulk 12.7C-wt\% and EMPA's pre-HHT average trend which shows a lower $\mathrm{C}-\mathrm{wt} \%$. Figure 4-15 shows dark spherical regions rich in carbon that would have been included in the bulk carbon analysis but not the EMPA result. Therefore the $\mathrm{C}$-wt $\%$ for the WC matrix follows the EMPA trend, average $7.75 \mathrm{wt} \%$, but the sample's C-wt $\%$ is $12.7 \%$. These rich carbon regions were probably introduced during the samples fabrication. There is a large difference in the pre- and post-HHT density. The pre-HHT density is $15.873 \mathrm{~g} / \mathrm{cm}^{3}$ while the post-HHT sample is $14.141 \mathrm{~g} / \mathrm{cm}^{3}$. This gives a percent difference of $10.91 \%$. 
The XRD spectrum shows that there were significant changes in phase. The pre-HHT sample in figure 14-18 illustrates that only the WC phase is present. The post-HHT sample reveals the presence of two phases: $\mathrm{WC}$ and $\mathrm{W}_{2} \mathrm{C}$. The presence of the $\mathrm{W}_{2} \mathrm{C}$ is evidence that the carbon-to-metal ratio has decreased due to depletion of carbon from the microstructure surface.

\subsubsection{Zirconium Carbide}

The EDS for the ZrC pre- and post-HHT samples are displayed figure 4-19. The results show that both samples' composition is consistent throughout the experiment. The difference in peak intensity is a function of the dead-time of the detector and has nothing to do with a change in composition. Figure 4-20a and 4-20b shows that on the surface the post-HHT sample displays little to no changes when compared to the pre-HHT sample. There is a slight discoloration caused by hot hydrogen etching the carbonaceous or loose material on the surface. Figures 4-21 and 4-22 show that qualitatively the microstructure was unchanged. The BSE images in 4-23 and 4-24 show no compositional differences or any depleted carbon regions due to hydrogen flow.

A bulk carbon analysis confirmed the qualitative result of an unchanged post-HHT $\mathrm{ZrC}$ microstructure. Figure 4-25 illustrates the pre-HHT C-wt $\%$ valued at $16.450 \mathrm{wt} \%$ and the postHHT C-wt $\%$ valued at $16.775 \mathrm{wt} \%$. Though the post-HHT sample is higher, the values are within close statistical error. The theoretical $\mathrm{ZrC}$ carbon content is $11.634 \mathrm{wt} \%$. The EMPA in figure 4-26 shows that there was no depletion of carbon at the surface of the sample. The variability in data points are caused by surface roughness.

Changes in density could reveal the presence of hydrogen embrittlement. The pre-HHT

sample's density was $6.648 \mathrm{~g} / \mathrm{cm}^{3}$, while the post-HHT sample's density was $6.635 \mathrm{~g} / \mathrm{cm}^{3}$. The 
change in density from the control sample is $0.197 \%$. Given the precision of the immersion testing, the values of the pre- and post-HHT density are statistically the same.

The XRD analysis was the last test to confirm an unchanged microstructure. The pre and post-HHT ZrC samples illustrated in figure 4-27 show that the peak-to-peak positions are unmoved, indicating the structure underwent little to no change.

\subsection{Tri-Carbides}

Four uranium tri-carbide compositions were tested in a hot hydrogen environment. The carbon-to-metal ratio was fixed at 0.95 . The primary goal was to examine depletion of carbon from the microstructure, but the investigation in how differing the metal atom fraction could affect the microstructure's integrity was also studied. The four tri-carbide compositions were pre-determined by previous research [1].

Tri-carbide sample designations, compositions, and changes in density and mass are listed in table 4-1. All of the tri-carbides displayed a mass loss as a result of the two hour hot hydrogen test. This was expected to occur because of vaporization of material species from the surface. Figures 4-28 through 4-31 are optical microscope images of each tri-carbide before and after testing. No signs of surface degradation such as partial melting of individual carbide constituent or hydrogen induced blistering or cracking was observed.

\subsubsection{Bulk Carbon Analysis}

Bulk carbon analysis for each tri-carbide is given in figures 4-32 through 4-35. With the exception for of TRI-C4, the other specimens' post-HHT carbon content were within error of the pre-HHT carbon content and no change occurred from the flow of hydrogen. The decrease of TRI-C4's carbon content can be explained by observing TRI-C4's pre-HHT SEM image in figure 4-46. TRI-C4 showed excessive porosity, implying the specimen had not undergone significant densification before HHT. The post-HHT samples illustrates the specimen underwent 
a large degree of sintering due to exposure to the $2900 \mathrm{~K}$ temperature. Figure $4-35$ showed TRIC4's initial carbon content at $11.775 \%$. The high carbon content was most likely due to carbonaceous material contaminating the surface and filling the pores. Samples TRI-C1, TRI$\mathrm{C} 2$, and TRI-C3 showed no measurable decrease in carbon content implying little to no erosion or corrosion of carbon from the tri-carbide matrix occurred.

\subsubsection{XRD Analysis}

Another measure of changes in the microstructure would have been revealed through XRD. None of the post-HHT diffraction patterns in figures 4-36 through 4-39 showed the presence of different phases from that of the pre-HHT samples' diffraction pattern. The structure was considered to be a solid solution because the diffraction pattern did not exhibit the presence of segregated carbide constituents. Observing the XRD pattern of $\mathrm{NbC}, \mathrm{ZrC}$, and $\mathrm{UC} / \mathrm{UC}_{2}$ powders from a previous research, [1], the diffraction pattern of the tri-carbides verified that a solid solution of $(\mathrm{U}, \mathrm{Zr}, \mathrm{Nb}) \mathrm{C}$ exist in both the pre- and post-HHT specimens. No changes in phase or crystallographic orientation occurred as a result of the two hour test at $2900 \mathrm{~K}$.

\subsubsection{SEM/EDS}

Using SEM, compositional contrast and topographical images were obtained. Figures 4-40 through 4-47 are images of the specimens' microstructure. Heterogeneity of $\mathrm{Zr}$ and $\mathrm{Nb}$ in the microstructure could not be ascertained from BSE images due to the proximity of their atomic numbers. It can be inferred that had the pre- and post-HHT XRD patterns displayed $\mathrm{ZrC}$ and $\mathrm{NbC}$ spectra, the two elements would have been segregated in the microstructure. Knowing that this was not the case, $\mathrm{Zr}$ and $\mathrm{Nb}$ could be assumed to have a homogeneous distribution. Based on compositional contrast images, all the tri-carbides displayed no regions of depleted carbon or a gradient of uranium from the surface. In addition to SEM images, EDS was taken of each preand post-HHT sample to ensure no contamination was introduced to the sample during testing or 
analysis preparation. Qualitative elemental composition for each tri-carbide is shown in figures 4-48 through 4-51. 
Table 4-1. Uranium tri-carbide results

\begin{tabular}{c|c|c|c|c|c|c|c|c}
\hline $\begin{array}{c}\text { Experiment } \\
\text { Designation }\end{array}$ & $\begin{array}{c}\text { Tri-carbide composition } \\
\text { (atom fraction) }\end{array}$ & $\begin{array}{c}\text { Theoretical } \\
\text { Carbon } \\
\text { Content }(\mathrm{wt} \%)\end{array}$ & $\begin{array}{c}\text { Pre-HHT } \\
\text { Density } \\
\left(\mathrm{g} / \mathrm{cm}^{3}\right)\end{array}$ & $\begin{array}{c}\text { Post-HHT } \\
\text { Density } \\
\left(\mathrm{g} / \mathrm{cm}^{3}\right)\end{array}$ & $\begin{array}{c}\text { Density } \\
\text { difference } \\
\left(\mathrm{g} / \mathrm{cm}^{3}\right)\end{array}$ & $\begin{array}{c}\text { Pre-HHT } \\
\text { Mass } \\
(\mathrm{g})\end{array}$ & $\begin{array}{c}\text { Post-HHT } \\
\text { Mass } \\
(\mathrm{g})\end{array}$ & $\begin{array}{c}\text { Mass } \\
\text { Difference } \\
(\mathrm{g})\end{array}$ \\
\hline TRI-C1 & $\left(\mathrm{U}_{0.1}, \mathrm{Zr}_{0.58}, \mathrm{Nb}_{0.32}\right) \mathrm{C}_{0.95}$ & 10.191 & 6.1066 & 5.7567 & $-0.3499^{*}$ & 2.24545 & 2.11287 & $-0.13258^{*}$ \\
\hline TRI-C2 & $\left(\mathrm{U}_{0.1}, \mathrm{Zr}_{0.68}, \mathrm{Nb}_{0.22}\right) \mathrm{C}_{0.95}$ & 10.206 & 6.5306 & 6.1129 & $-0.4117^{*}$ & 2.53249 & 2.43116 & $-0.10133^{*}$ \\
\hline TRI-C3 & $\left(\mathrm{U}_{0.1}, \mathrm{Zr}_{0.77}, \mathrm{Nb}_{0.13}\right) \mathrm{C}_{0.95}$ & 10.219 & 6.2807 & 6.4215 & $+0.1408^{*}$ & 2.85000 & 2.81581 & $-0.03416^{*}$ \\
\hline TRI-C4 & $\left(\mathrm{U}_{0.05}, \mathrm{Zr}_{0.62}, \mathrm{Nb}_{0.33}\right) \mathrm{C}_{0.95}$ & 10.866 & 6.2961 & 6.9031 & $+0.607^{*}$ & 4.83599 & 4.70004 & $-0.12561 *$ \\
\hline
\end{tabular}

* A negative value implies a loss occurred. A positive value implies a gain occurred 


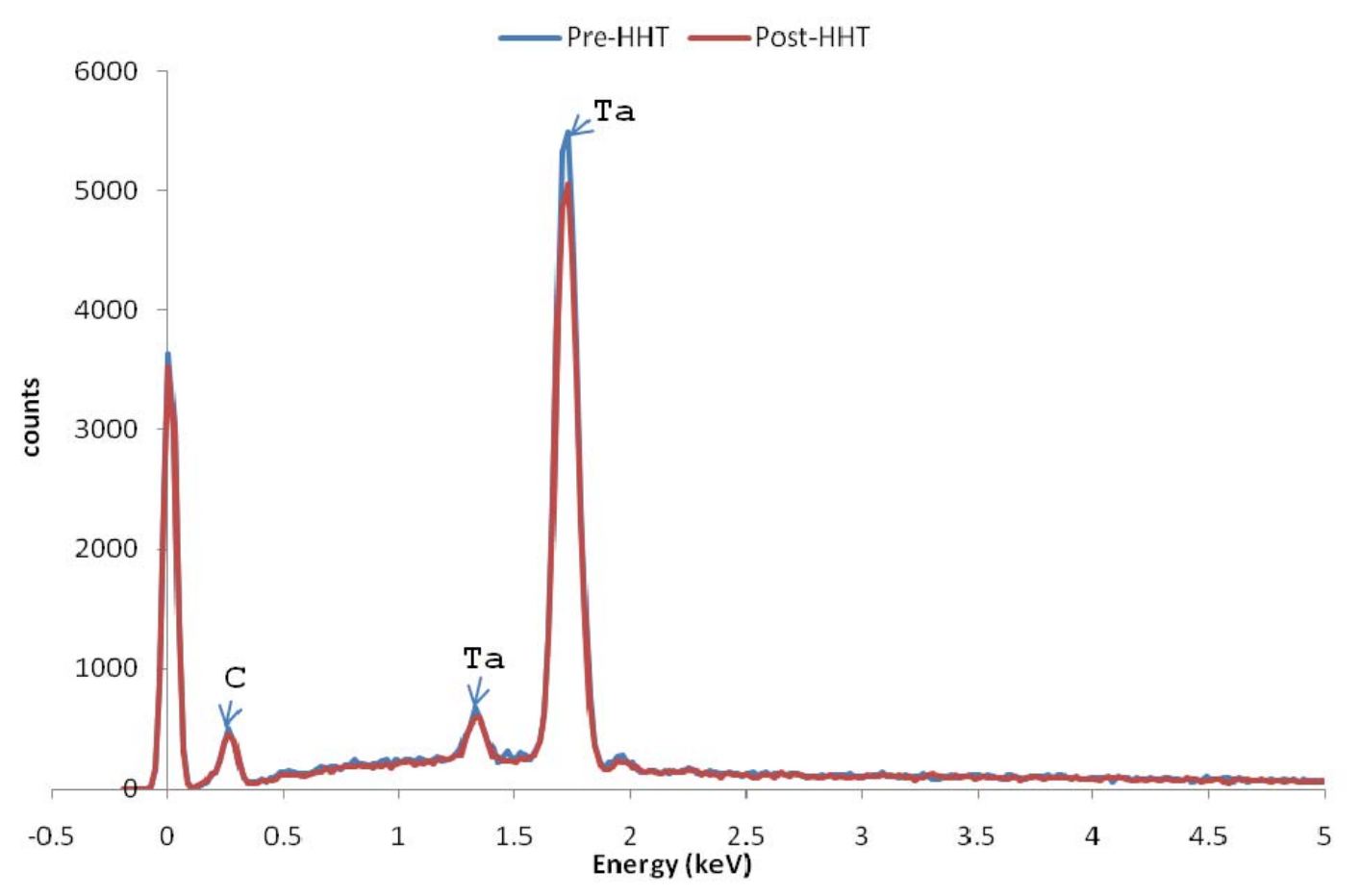

Figure 4-1. EDS of pre- and post-HHT TaC sample

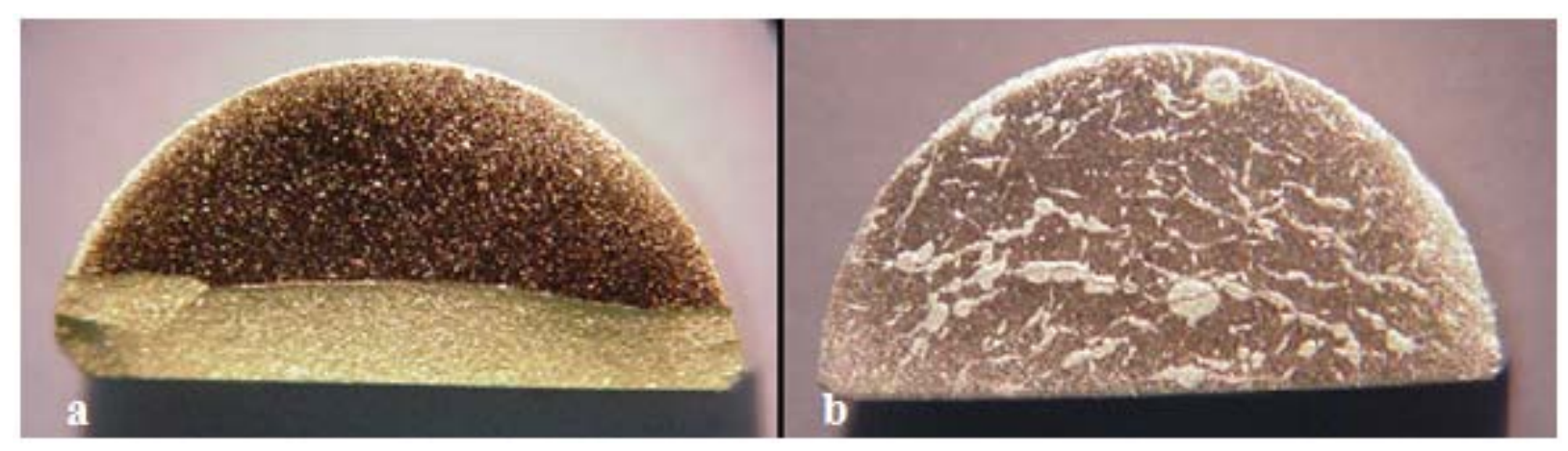

Figure 4-2. A) Pre-HHT TaC sample. B) Post-HHT TaC sample 


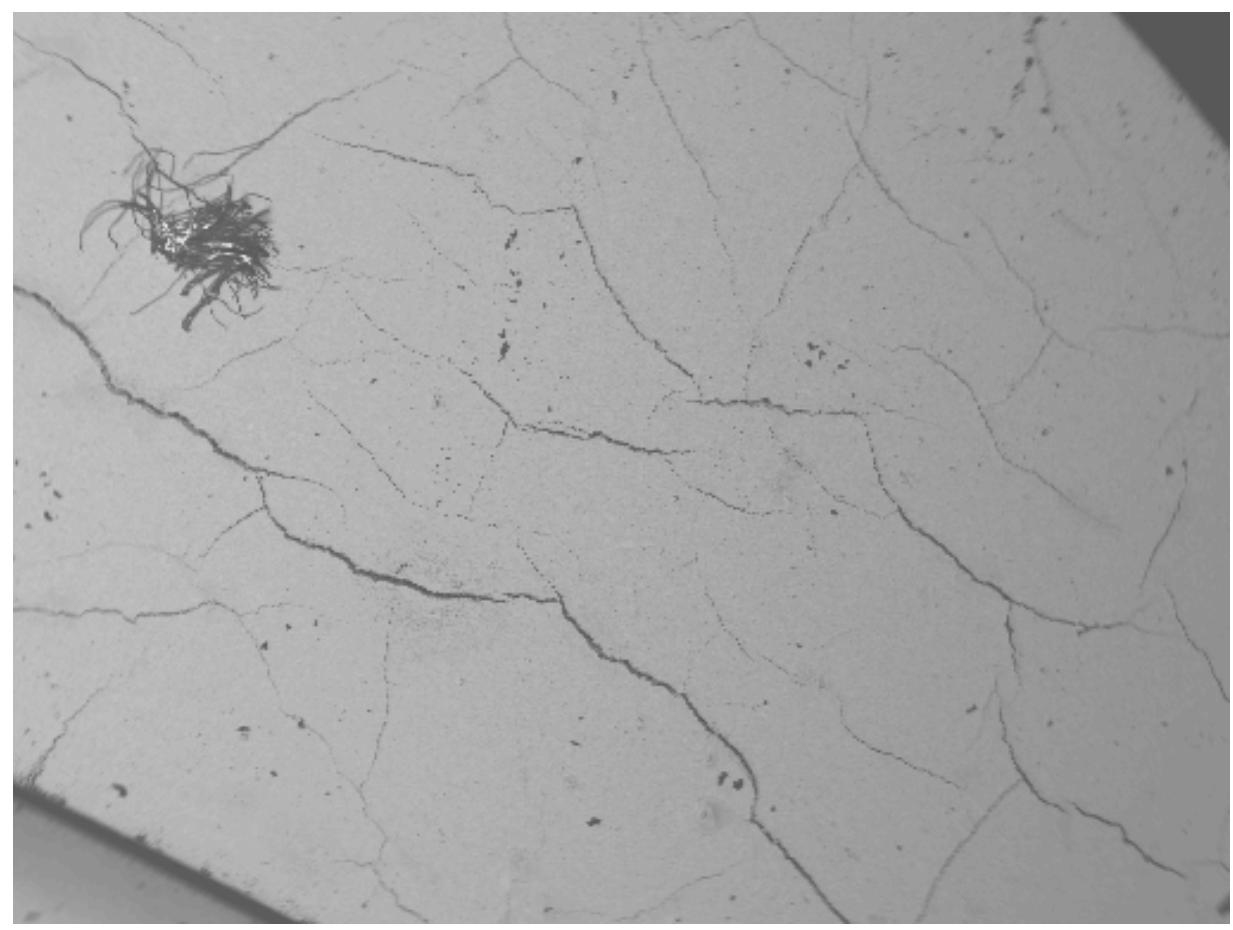

Figure 4-3. TaC BSE image displaying post-HHT cracking

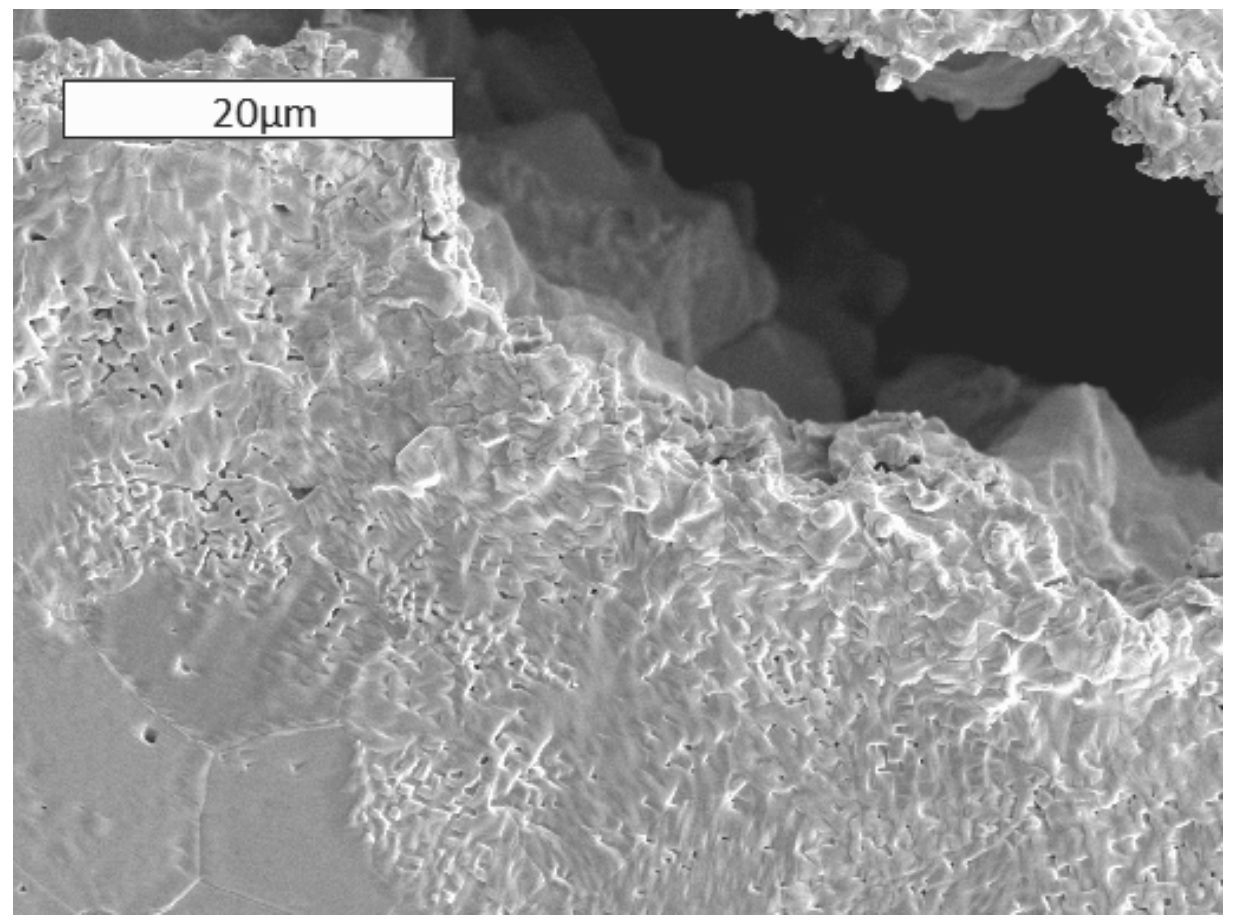

Figure 4-4. TaC SE image illustrating fracture zone 


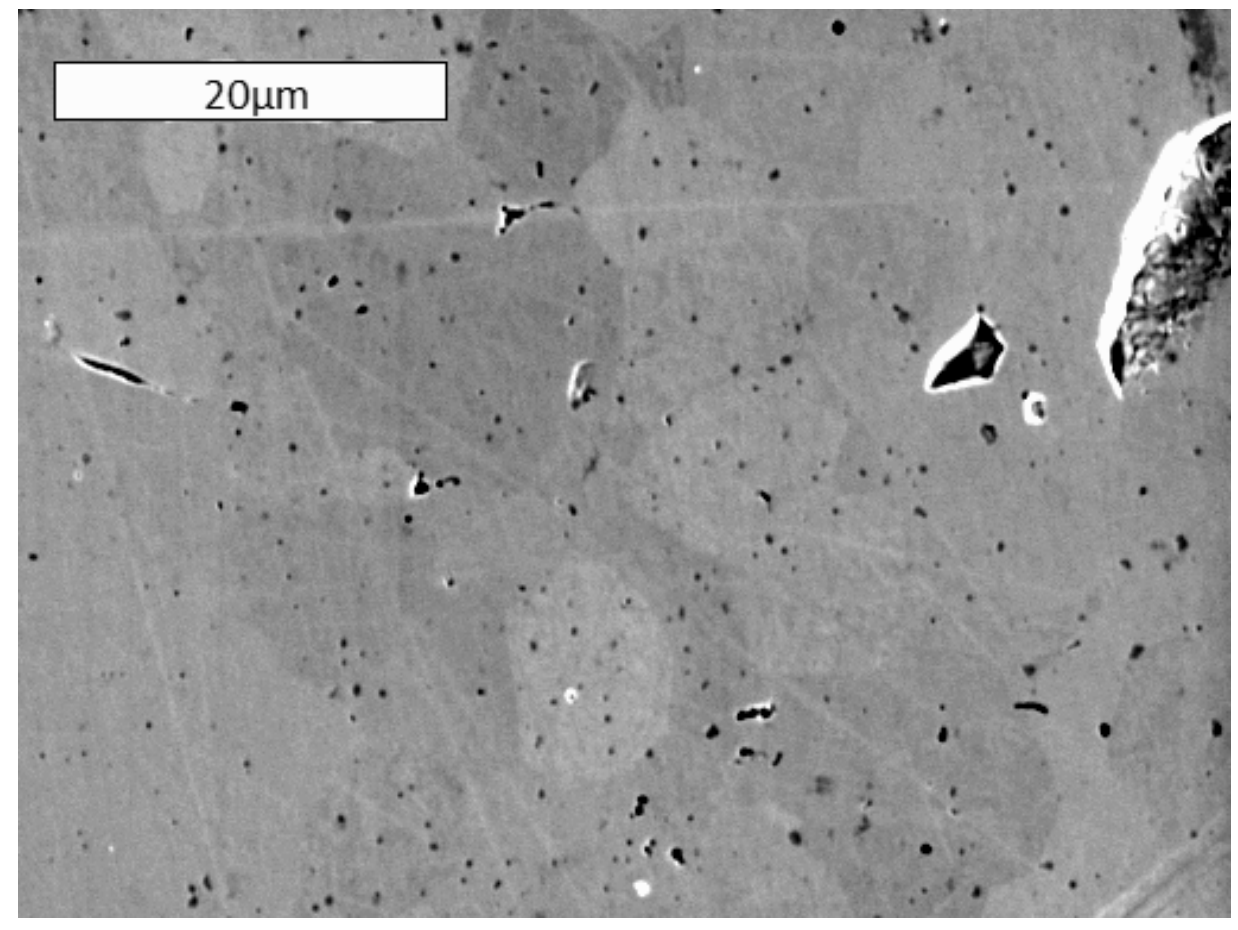

Figure 4-5. TaC SE image of pre-HHT specimen

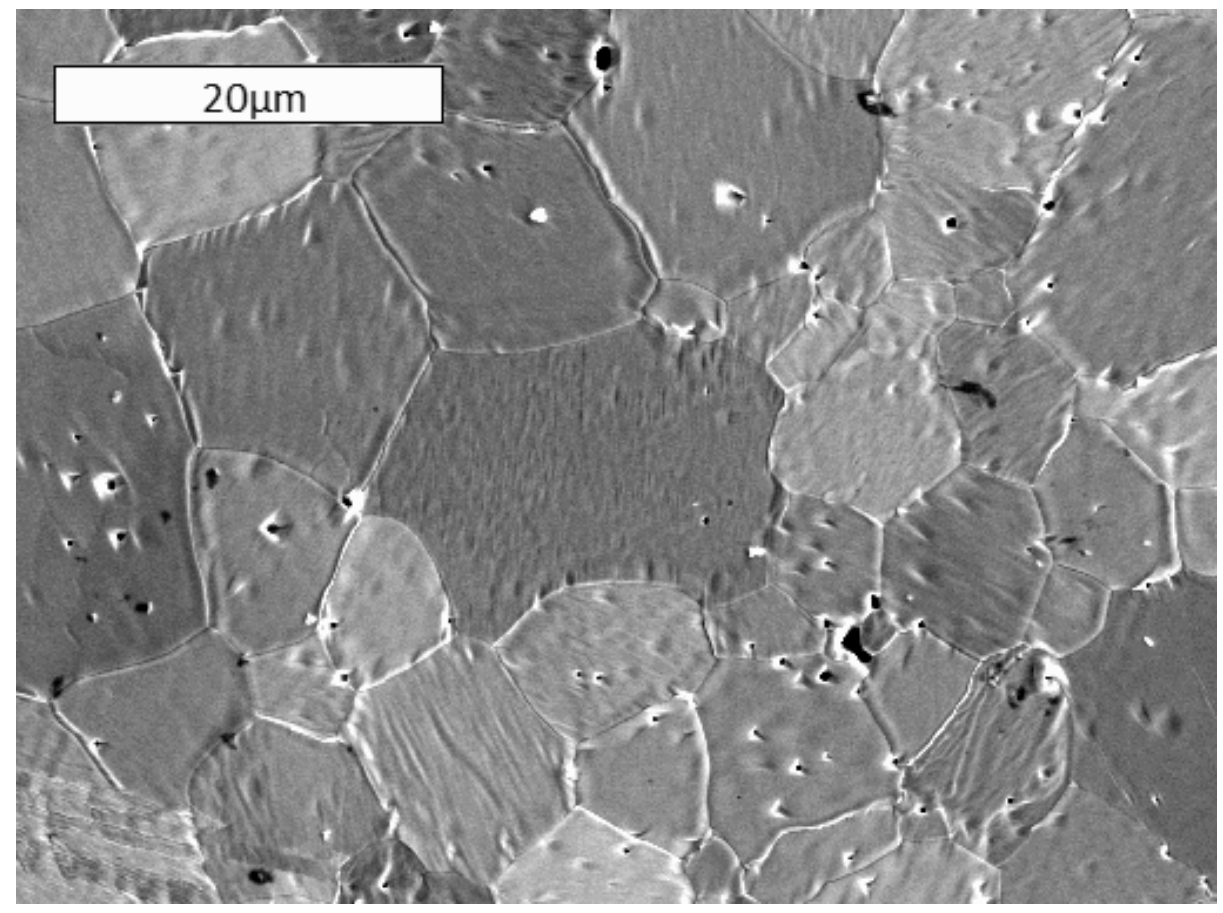

Figure 4-6. TaC SE image of post-HHT specimen 


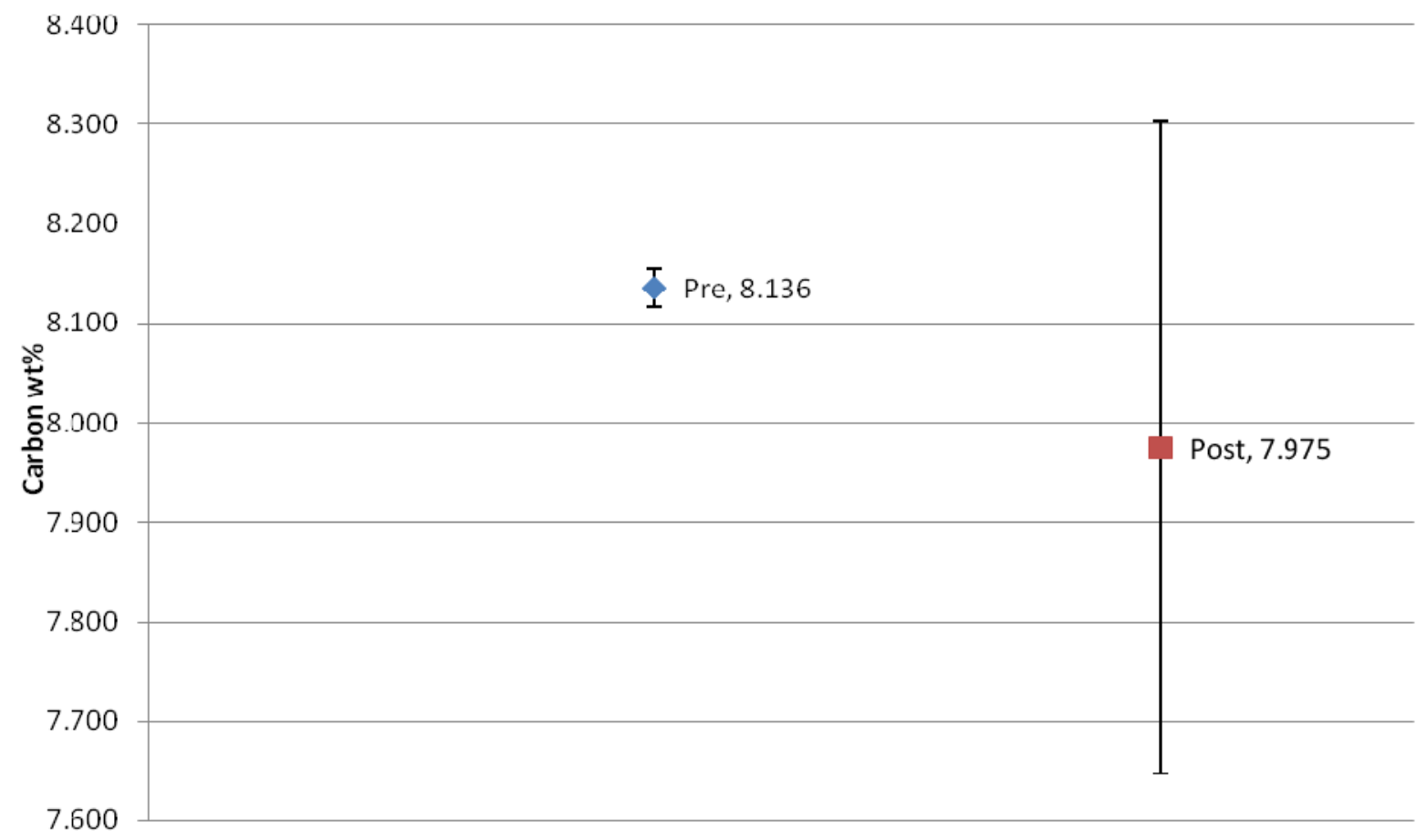

Figure 4-7. TaC bulk carbon content

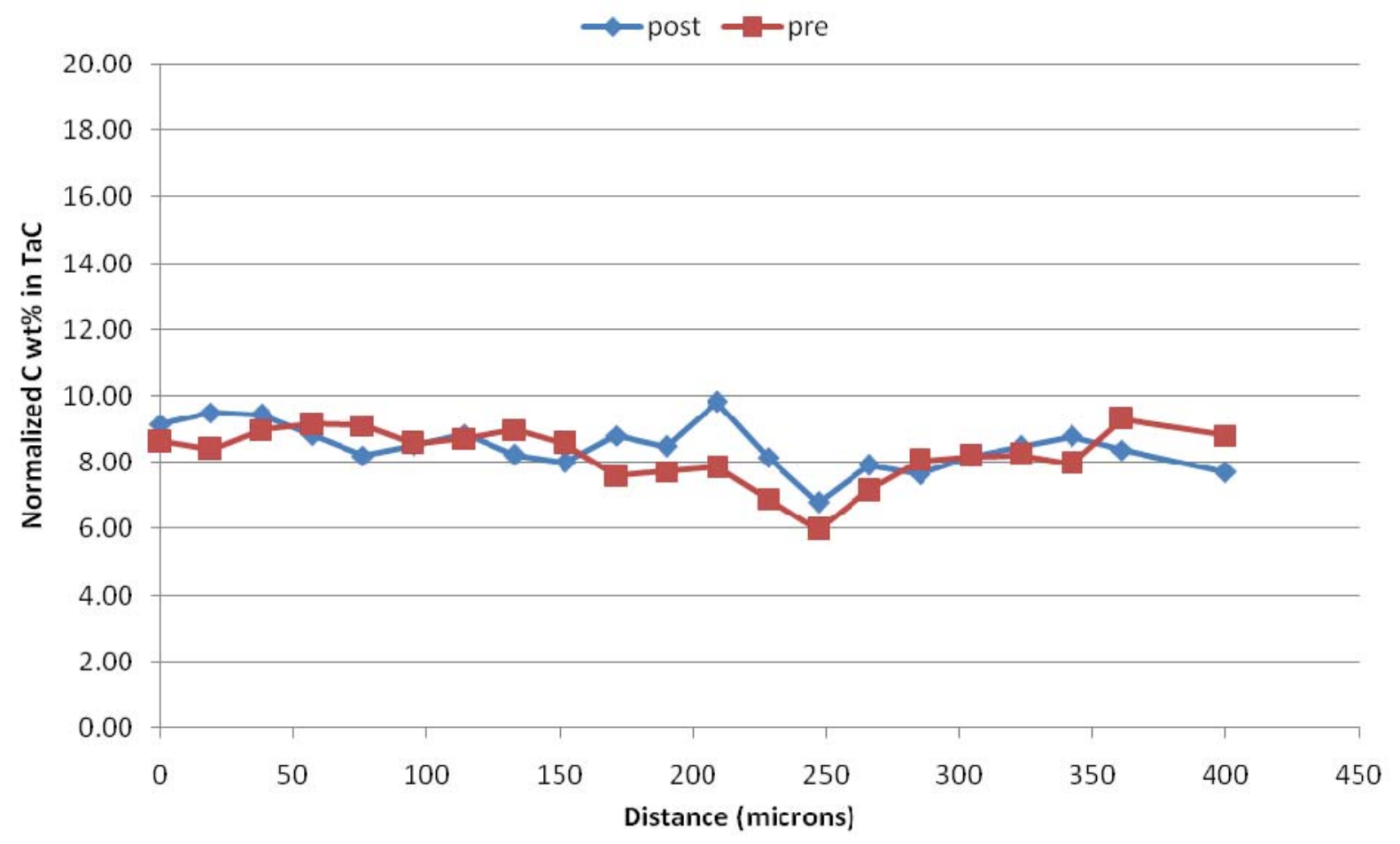

Figure 4-8. Mid-axial cross section showing TaC carbon content as a function of depth 


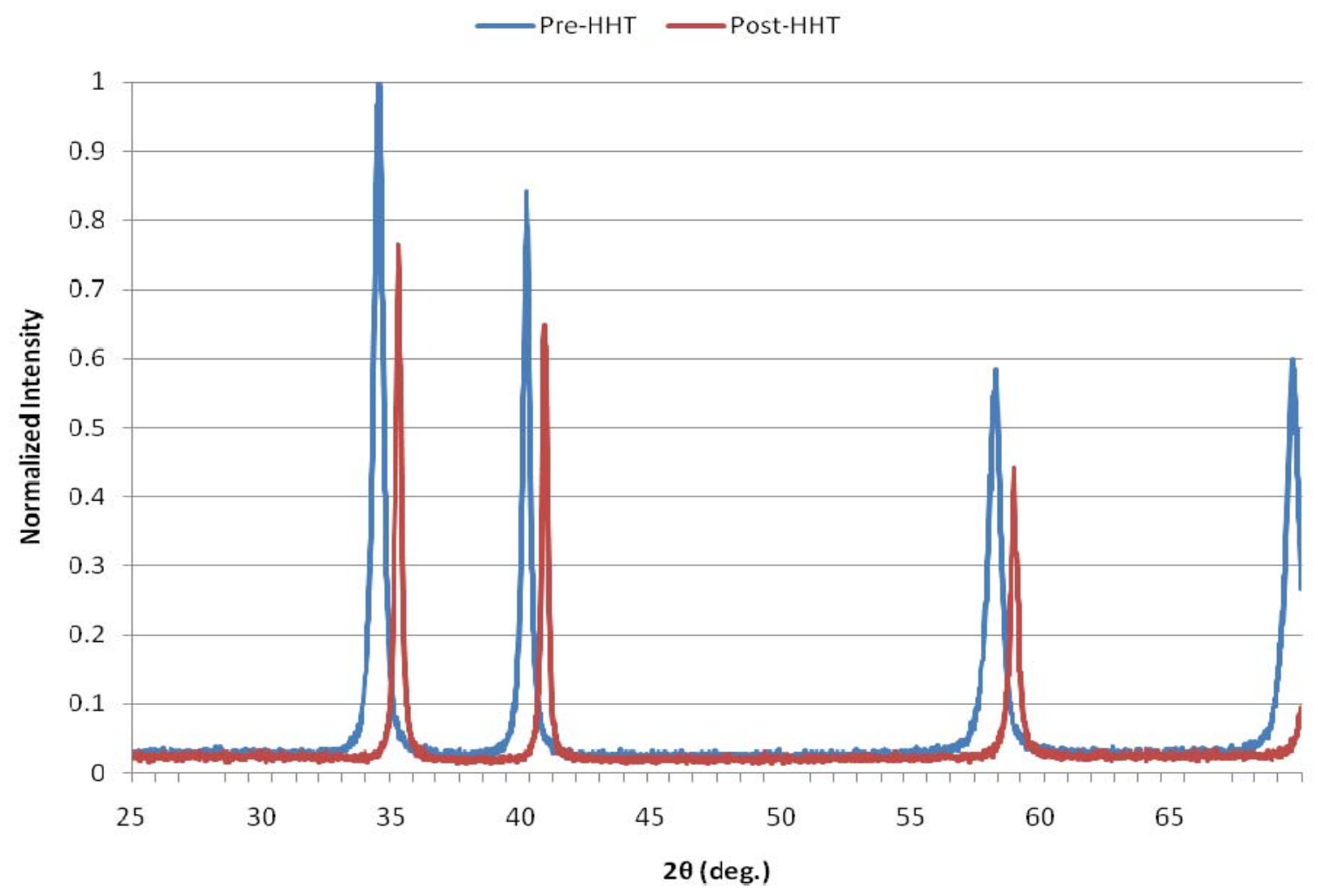

Figure 4-9. TaC pre- and post-HHT XRD spectrum

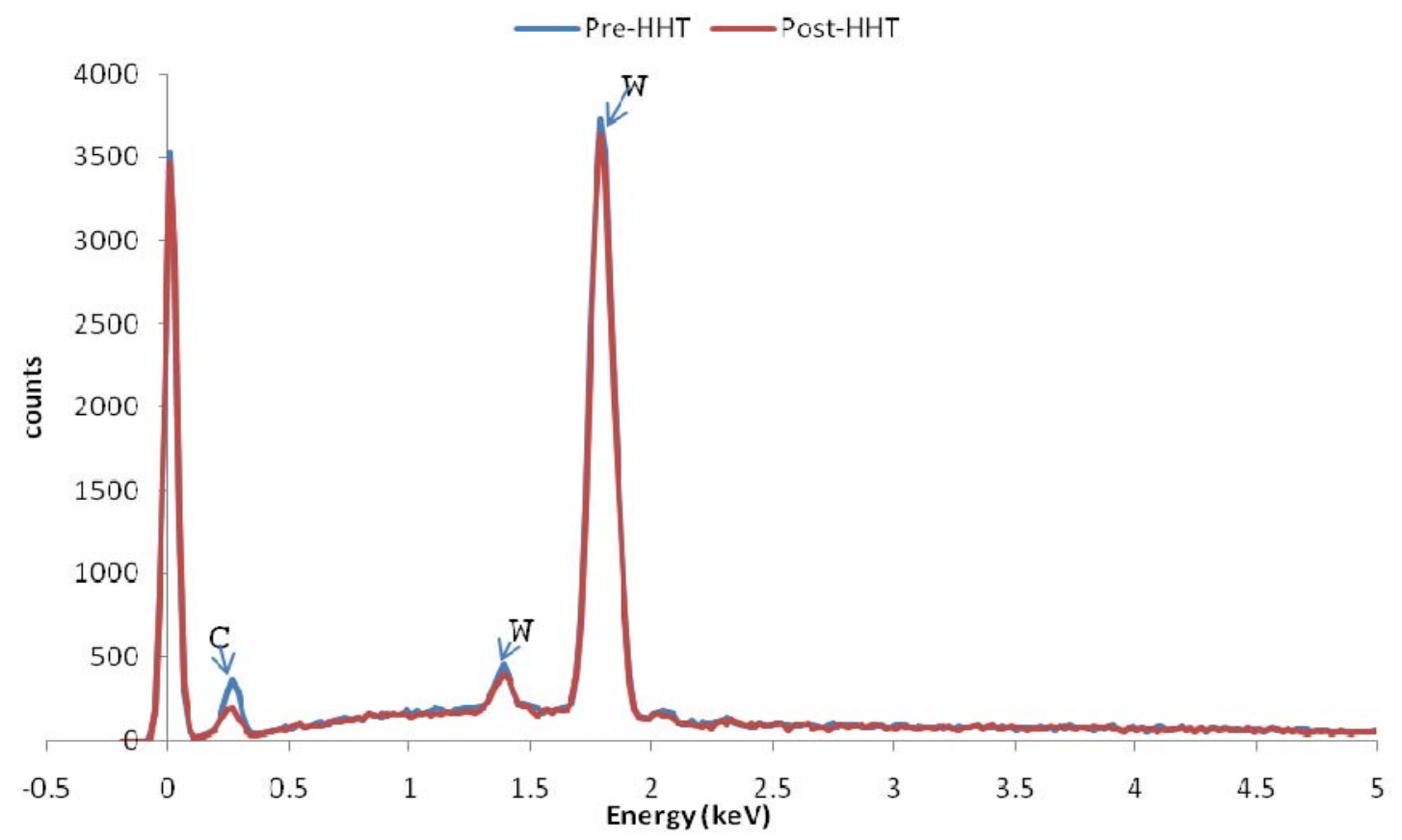

Figure 4-10. EDS of pre- and post-HHT of WC sample 

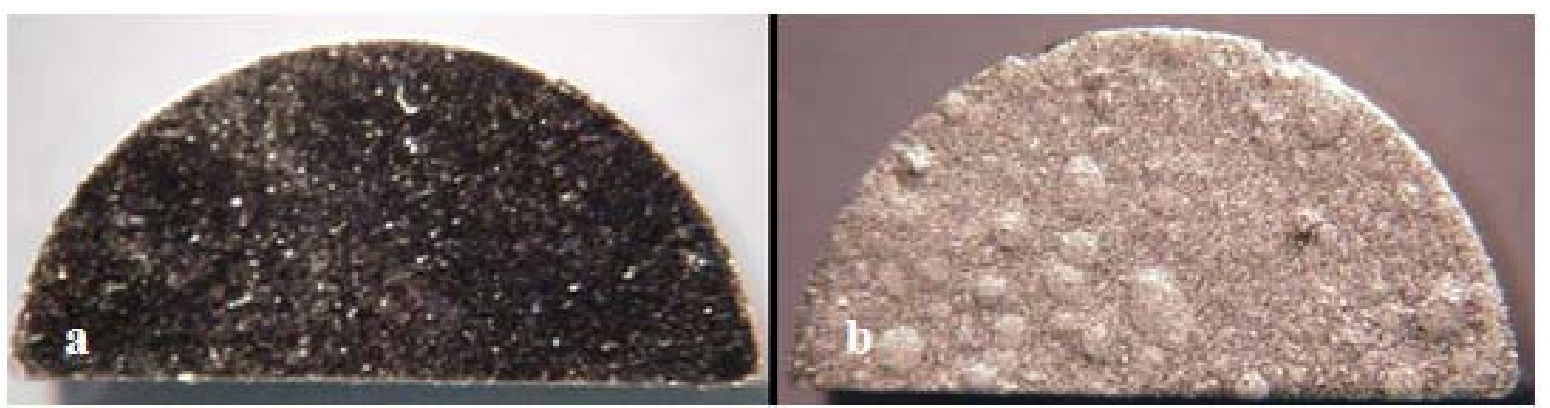

Figure 4-11. A) Pre-HHT WC sample. B) Post-HHT WC sample

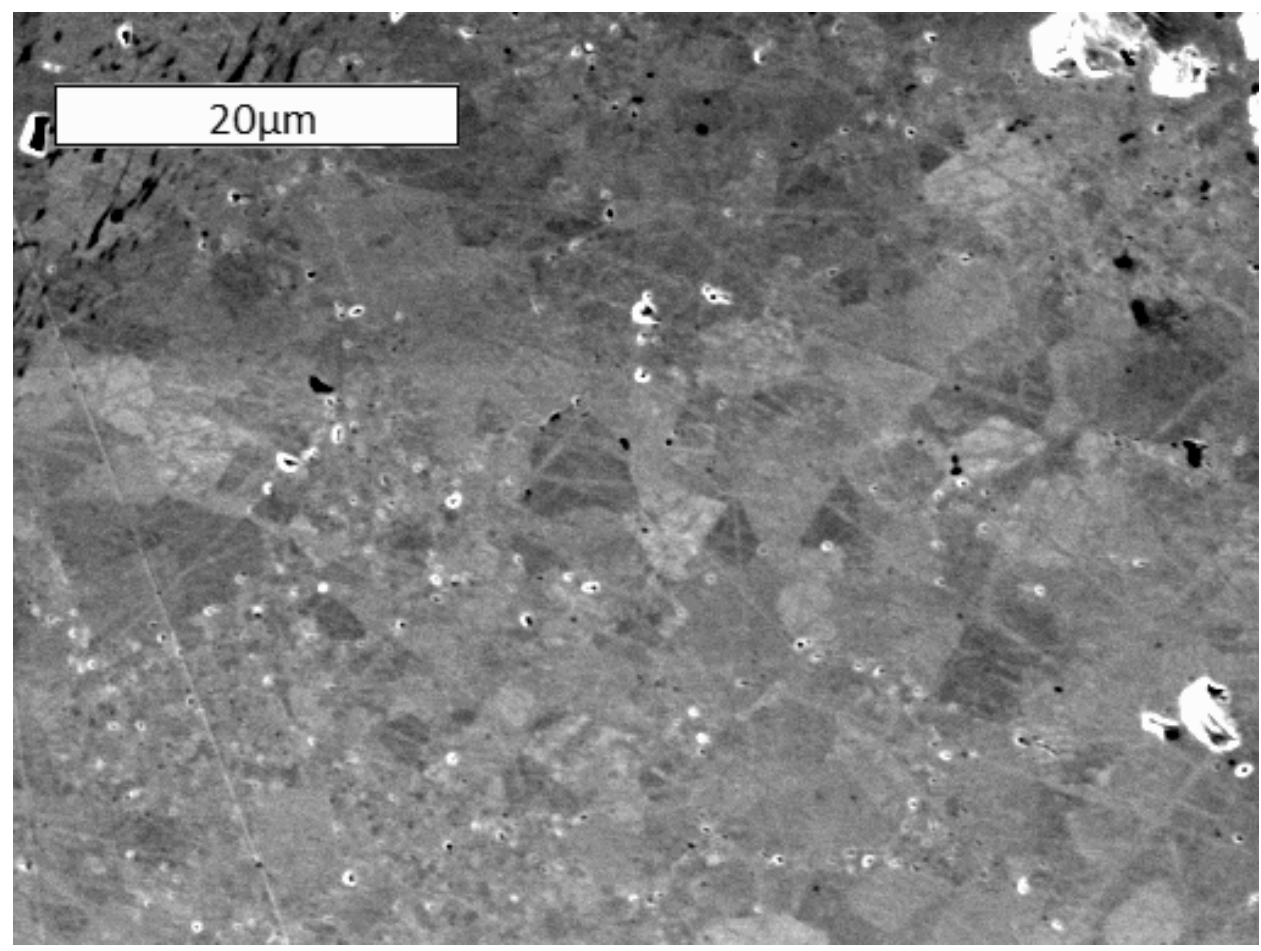

Figure 4-12. WC SE image of pre-HHT specimen 


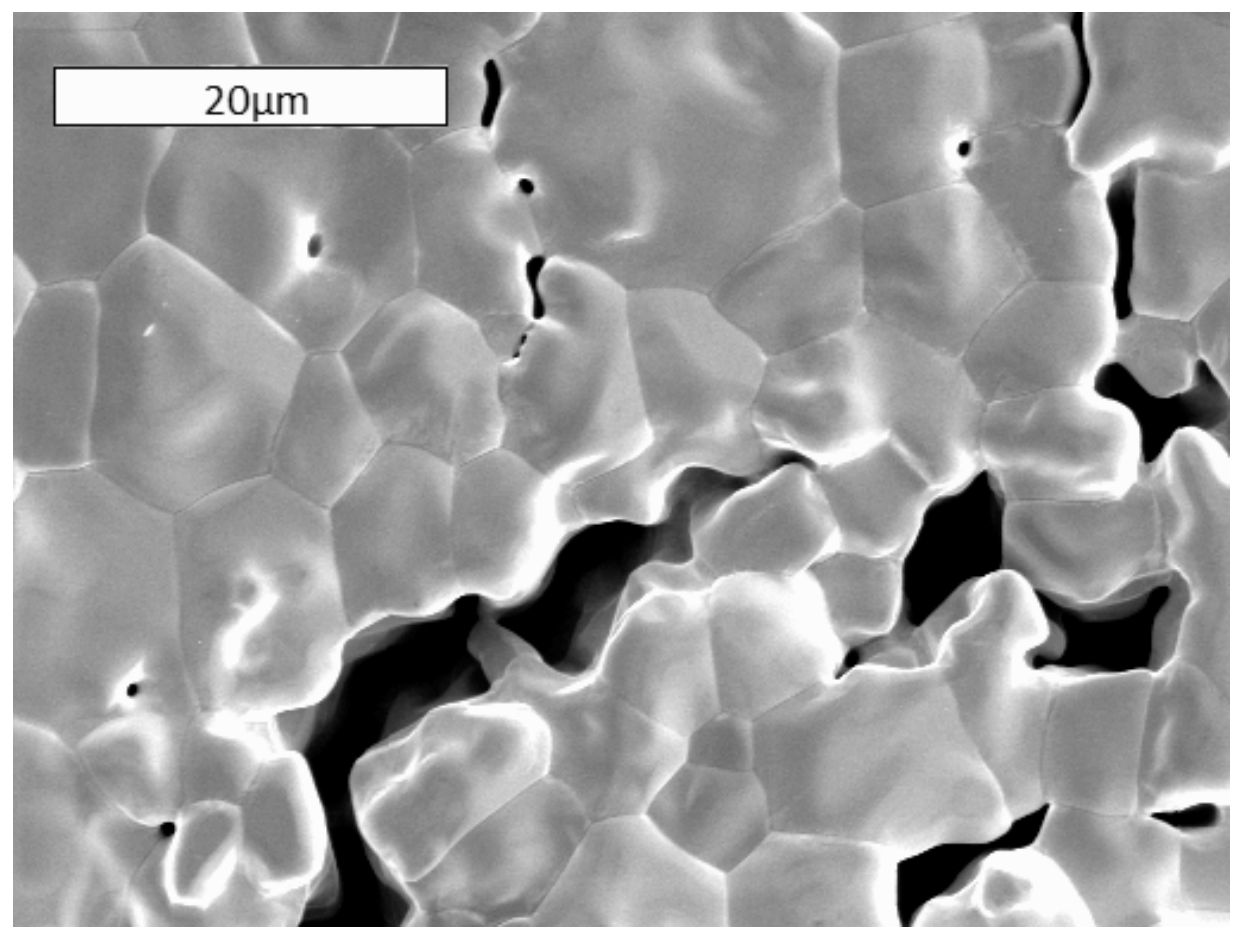

Figure 4-13. WC SE image of post-HHT specimen

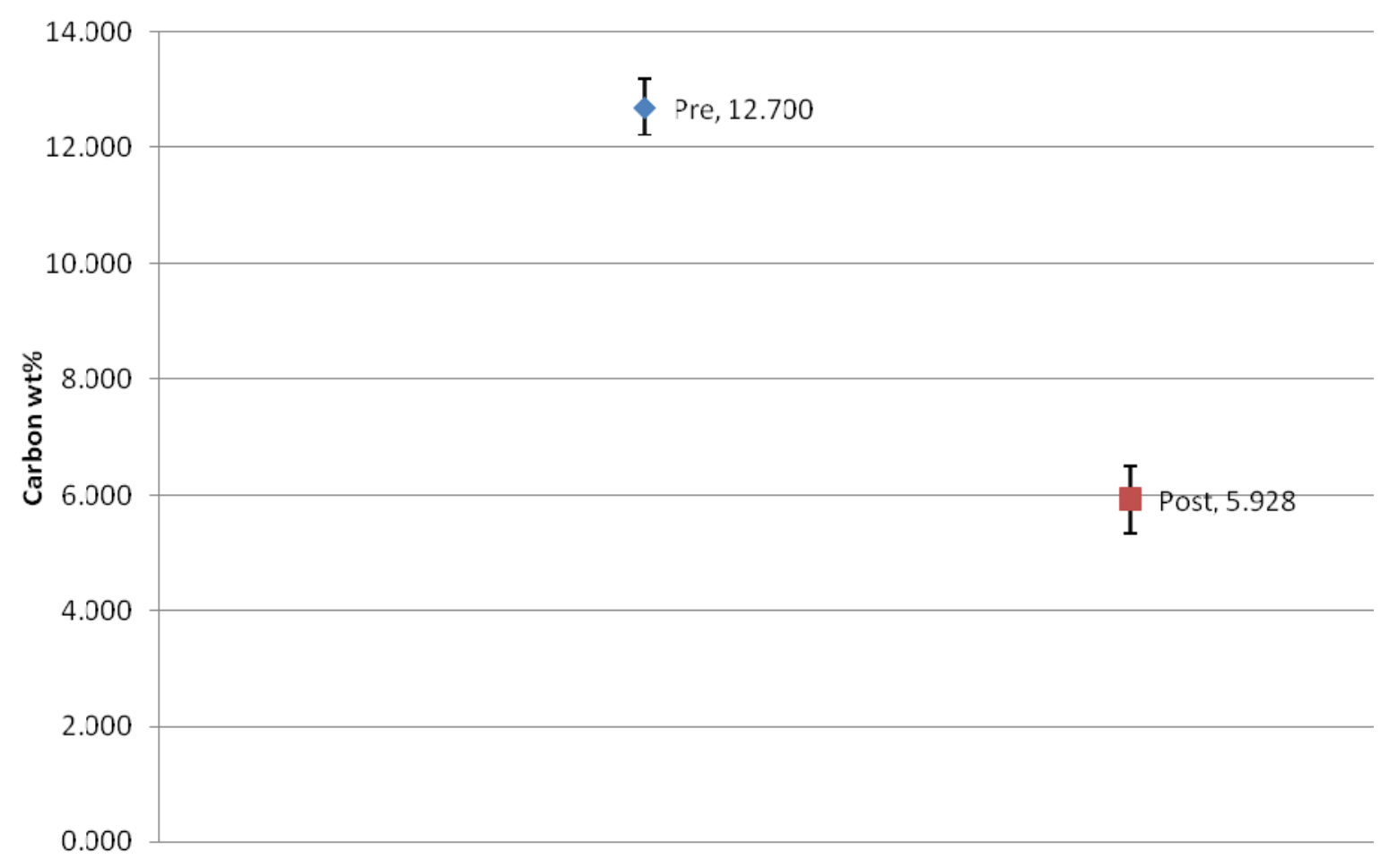

Figure 4-14. WC bulk carbon content 


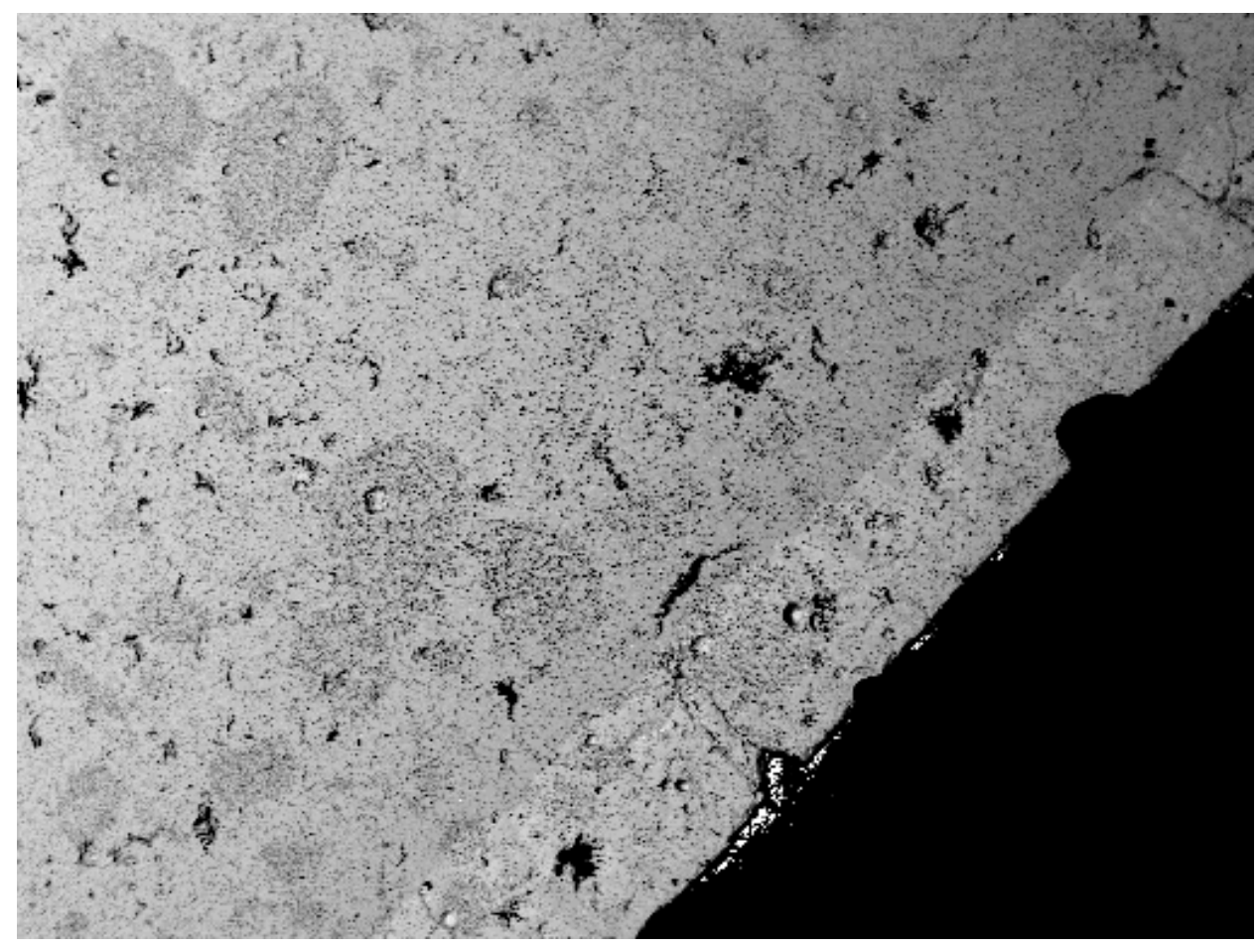

Figure 4-15. WC BSE image illustrating the transition zone where carbon depletion occurred

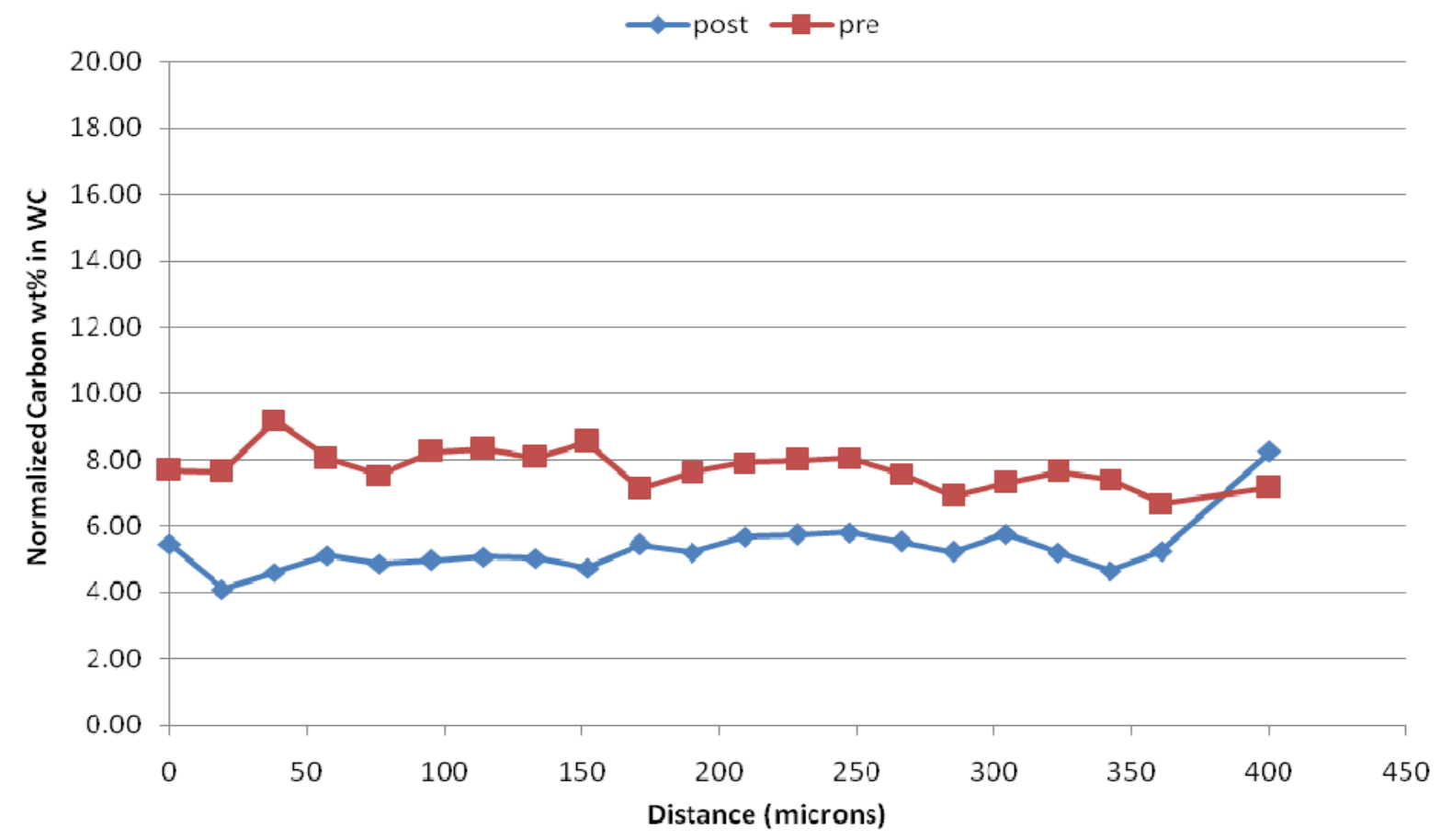

Figure 4-16. Mid-axial cross section showing WC carbon content as a function of depth 


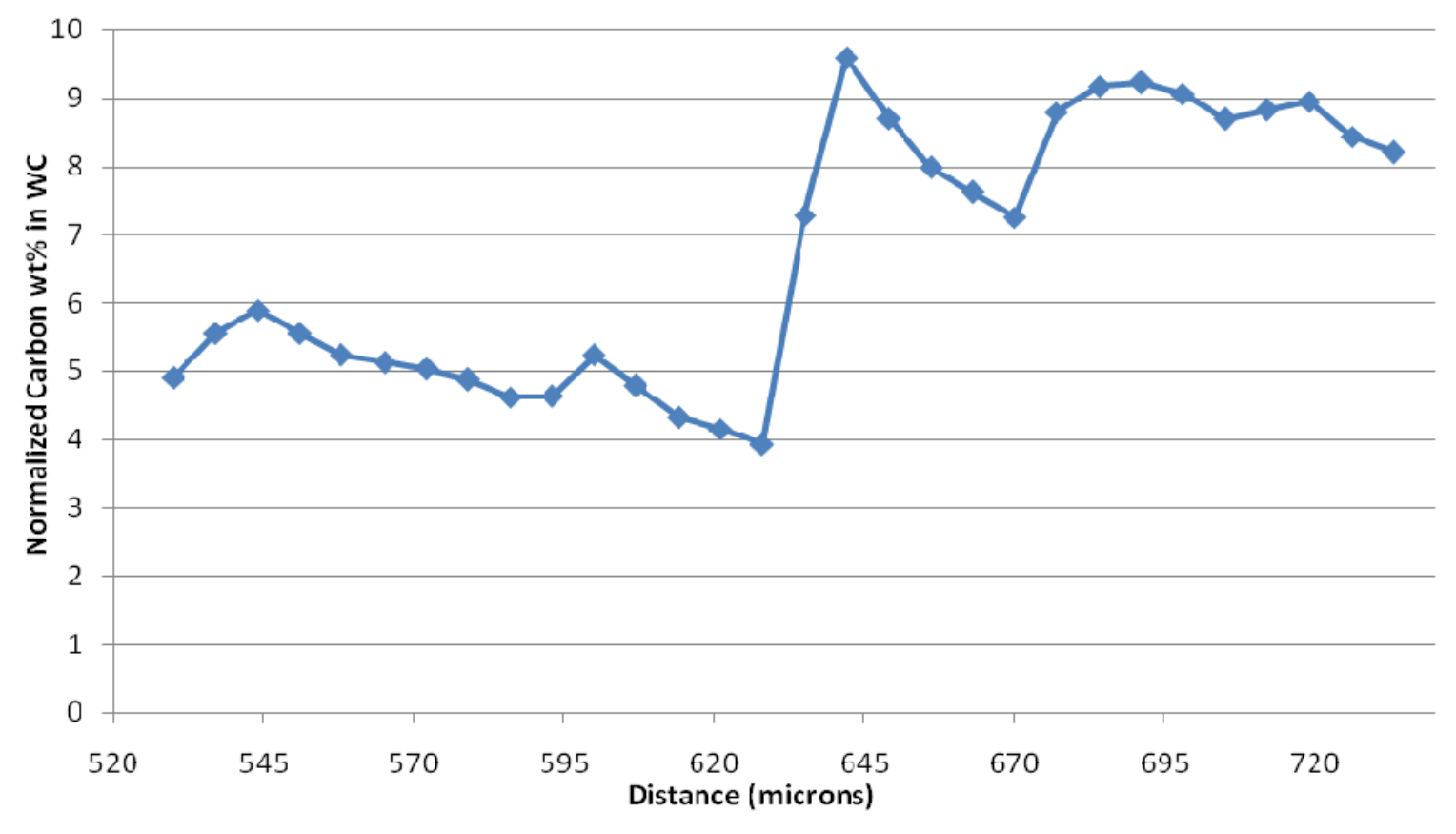

Figure 4-17. Mid-axial cross section of post-HHT showing WC transition zone of carbon content

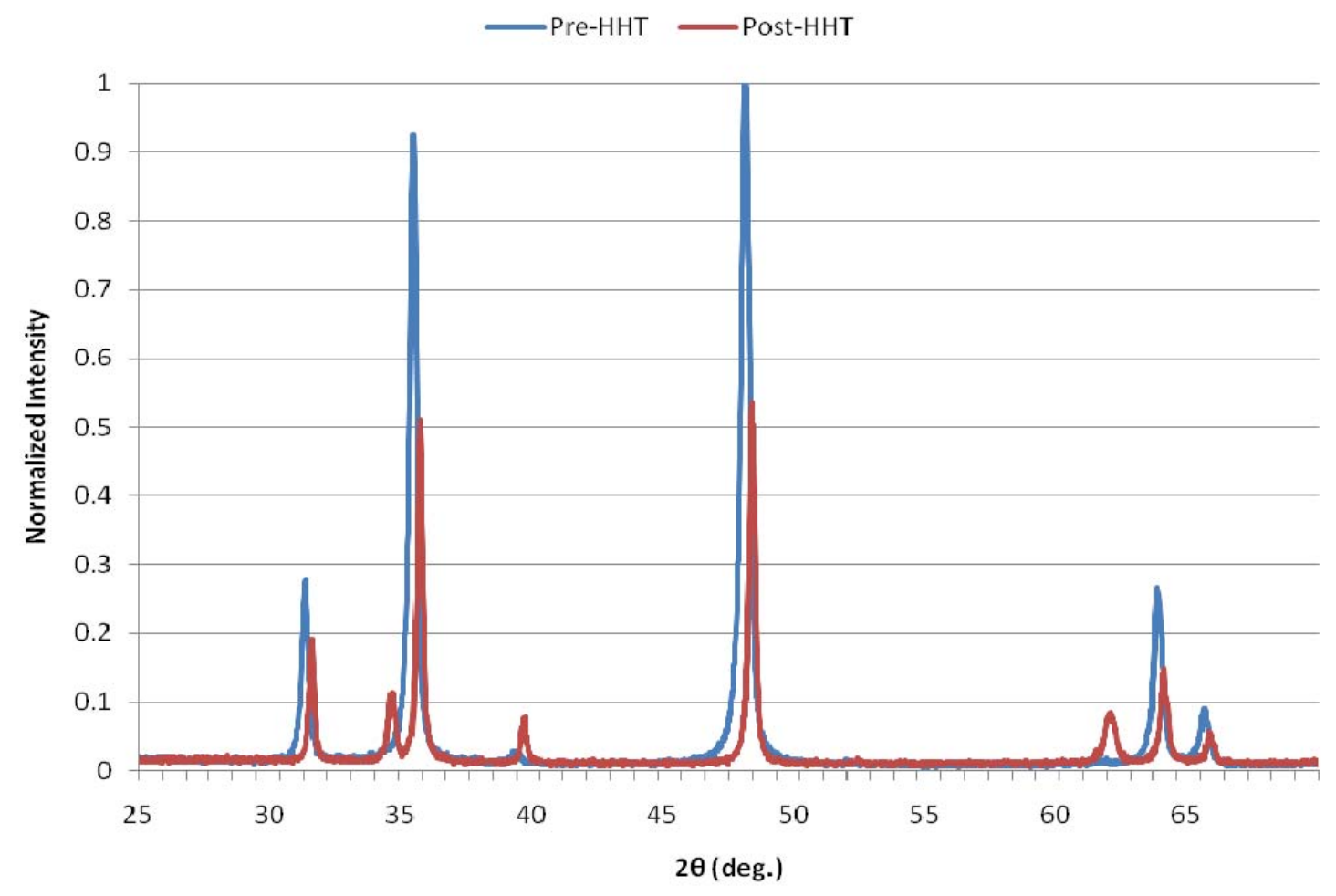

Figure 4-18. WC pre and post-HHT XRD spectrum 


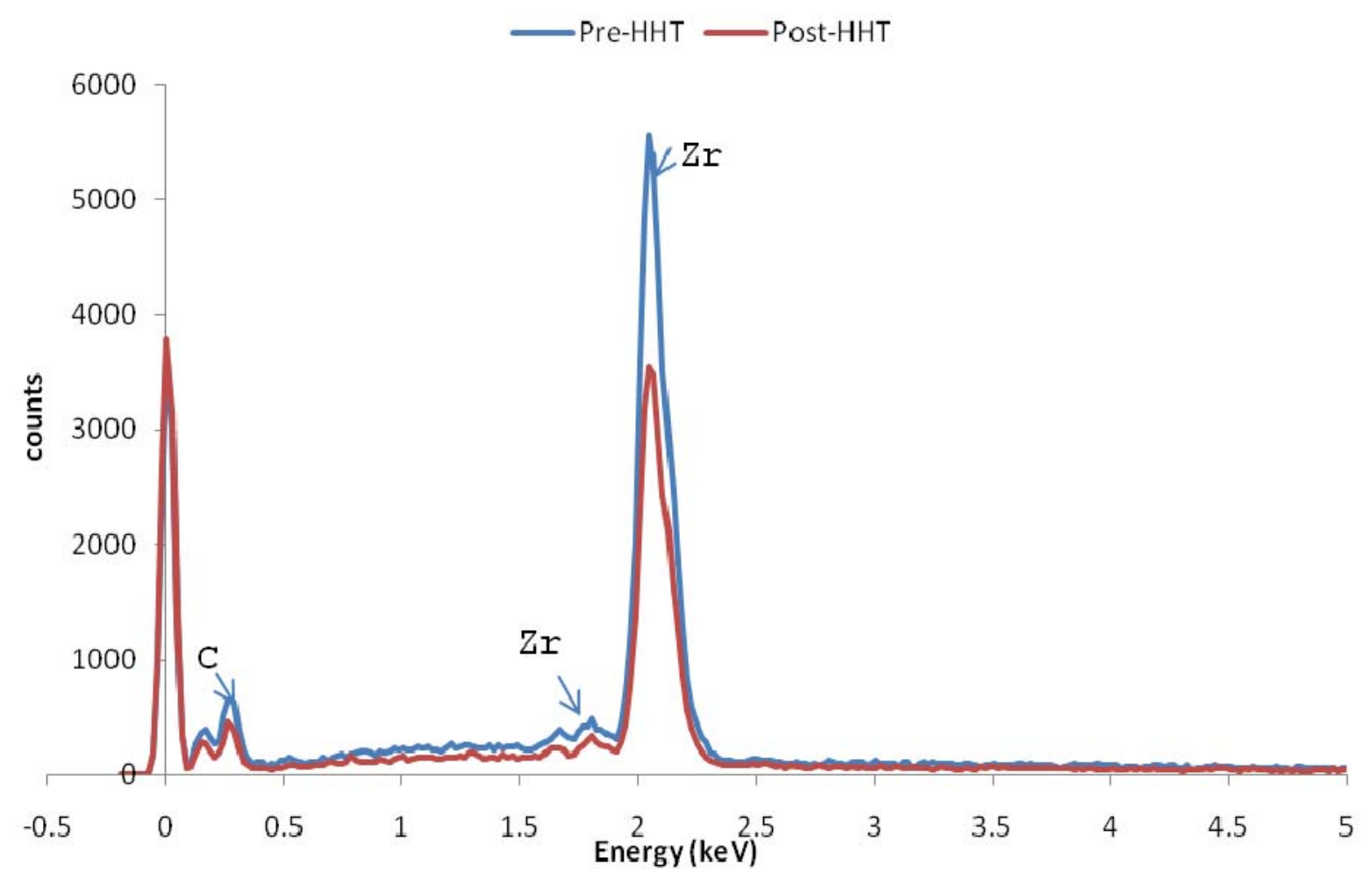

Figure 4-19. EDS of pre- and post-HHT of ZrC sample

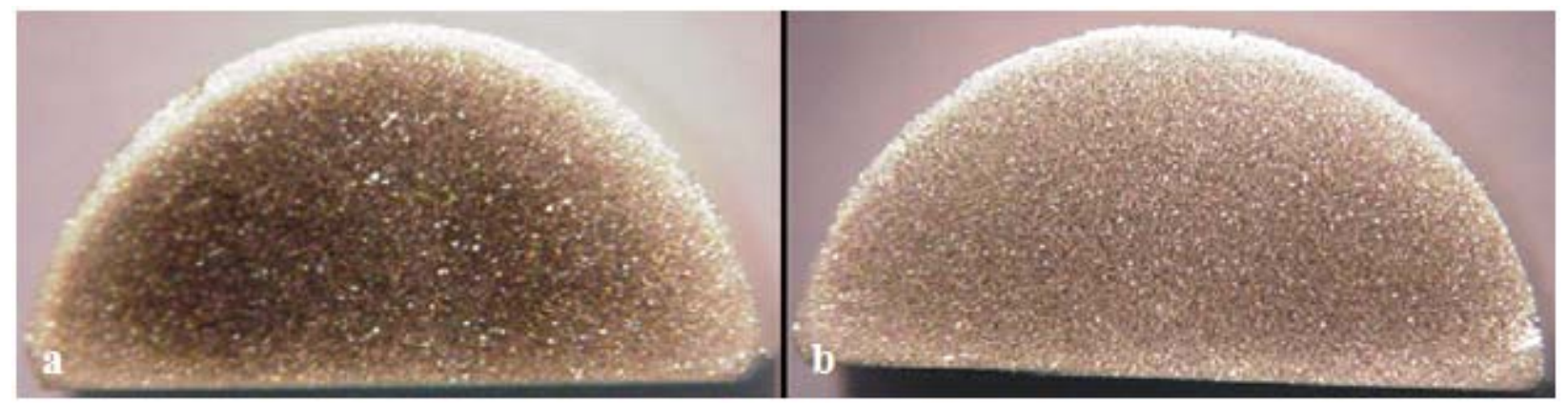

Figure 4-20. A) Pre-HHT ZrC sample. B) Post-HHT ZrC sample. 


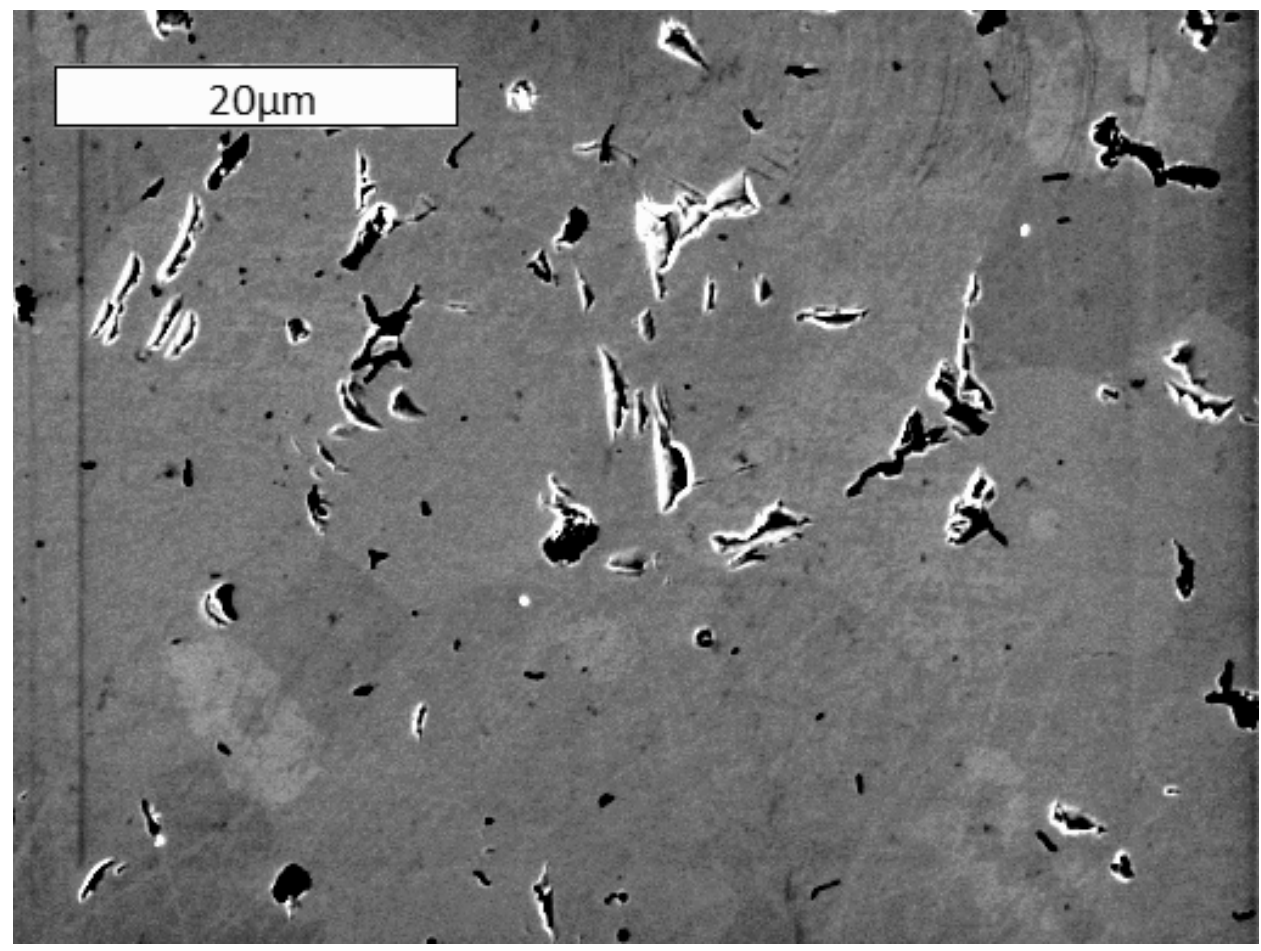

Figure 4-21. ZrC SE image of pre-HHT specimen

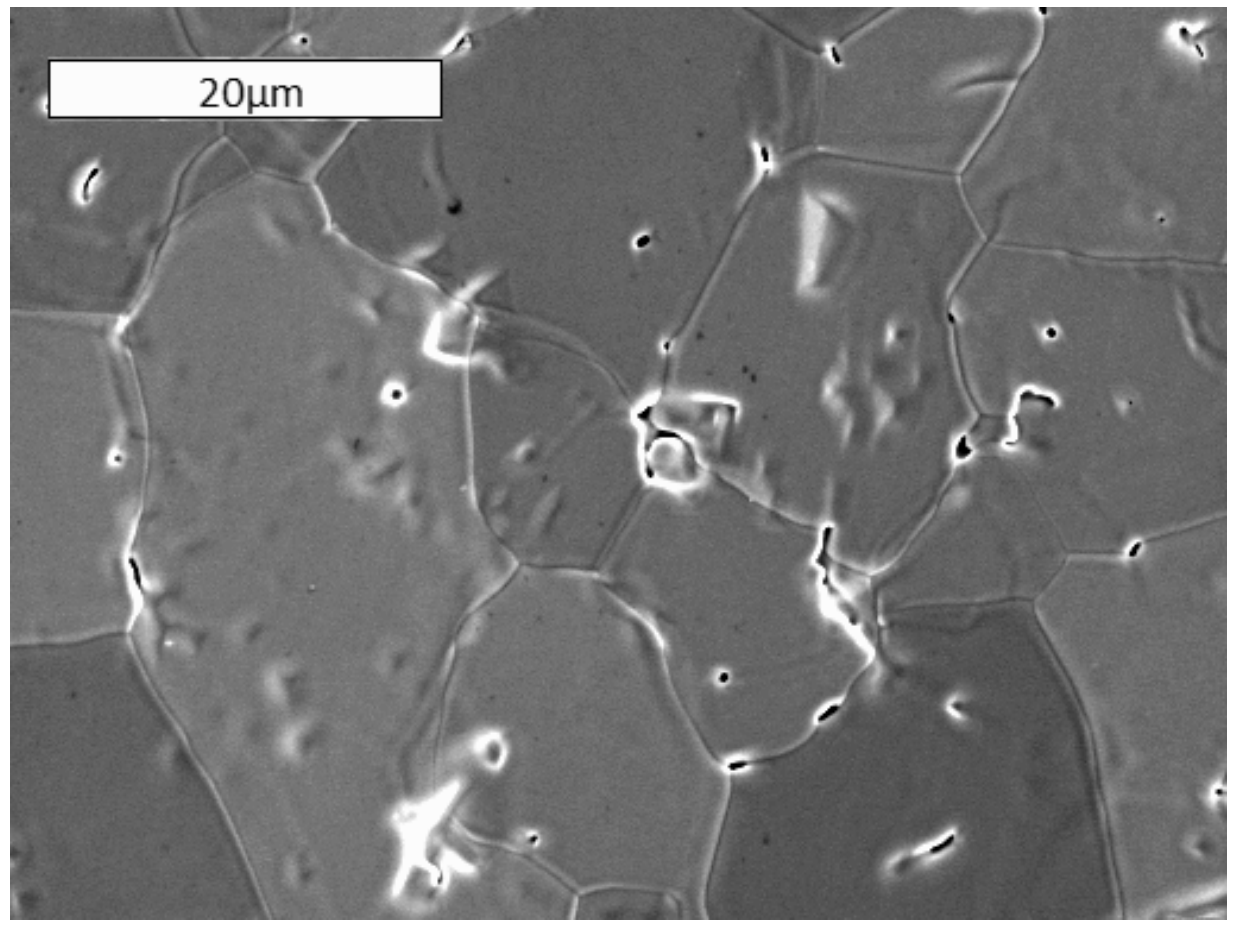

Figure 4-22. ZrC SE image of post-HHT specimen 


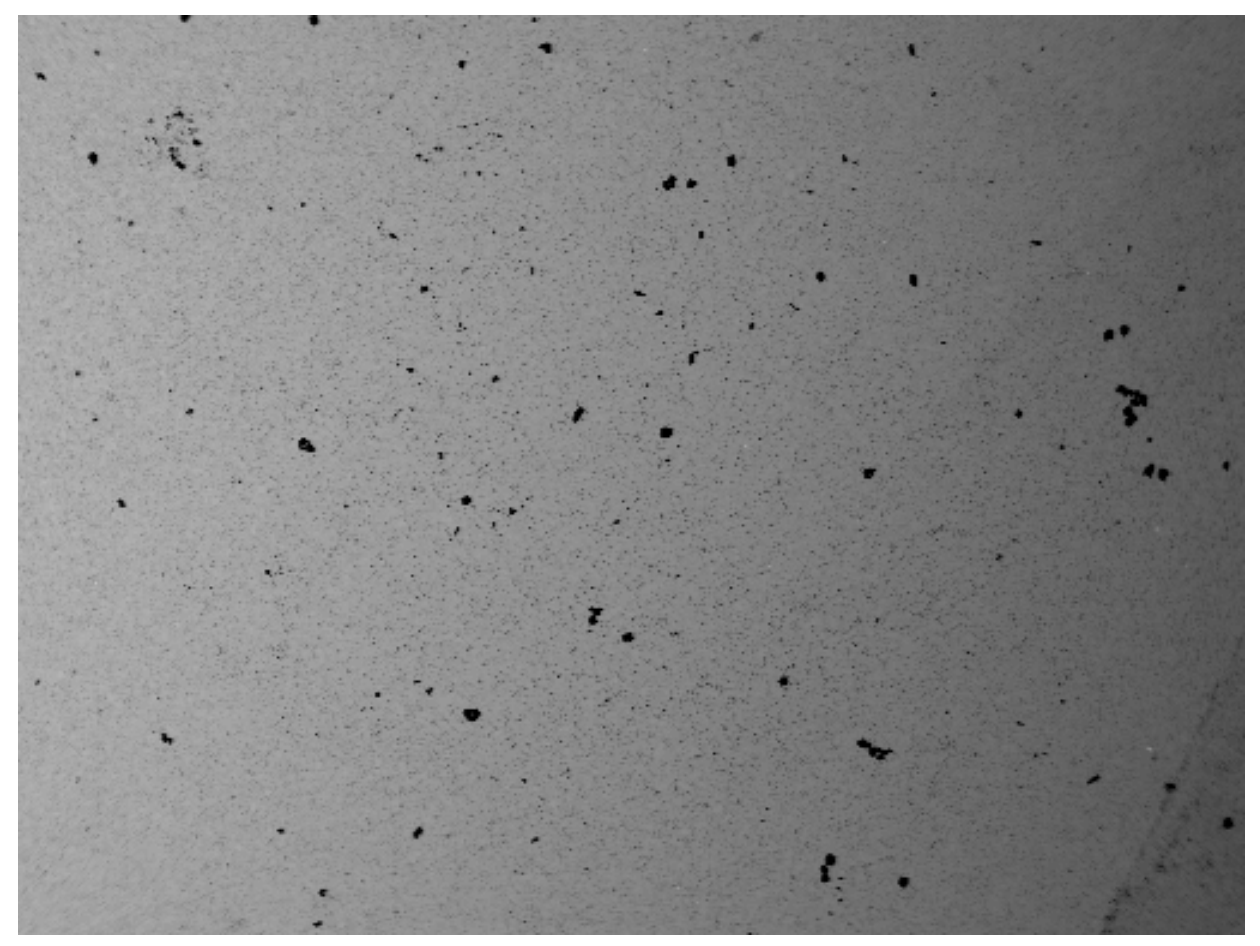

Figure 4-23. ZrC BSE image of pre-HHT specimen

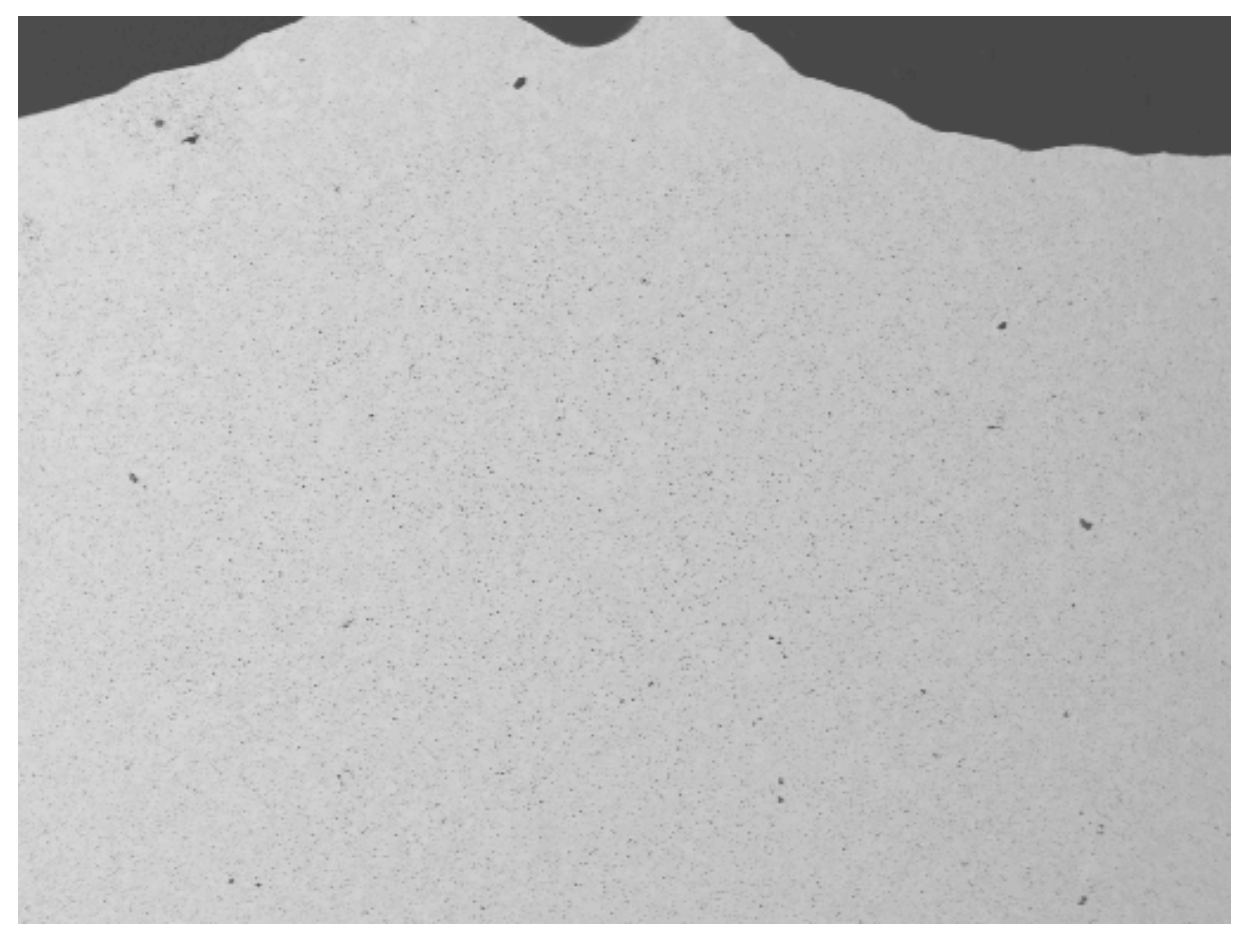

Figure 4-24. ZrC BSE image of post-HHT specimen 


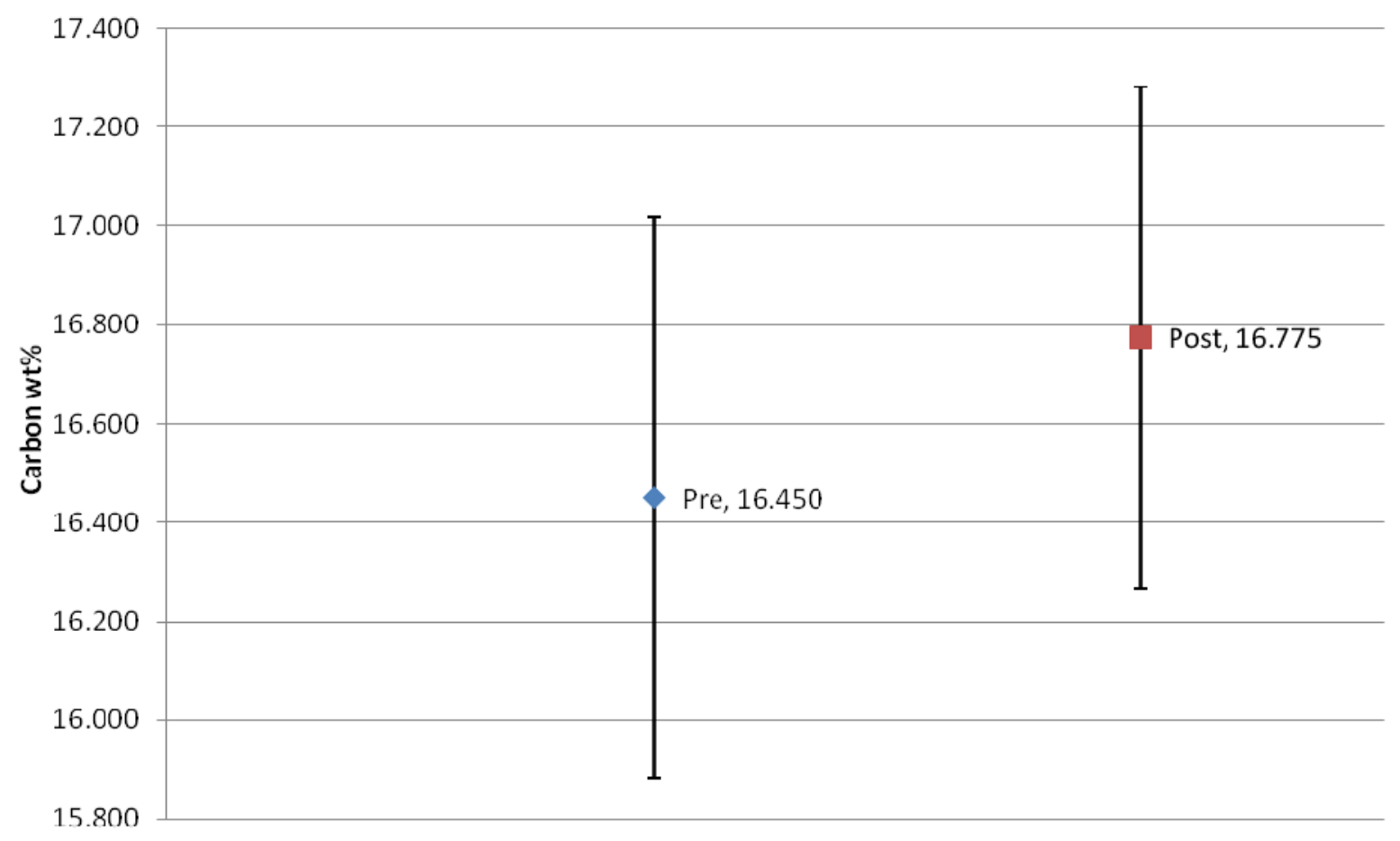

Figure 4-25. ZrC bulk carbon content.

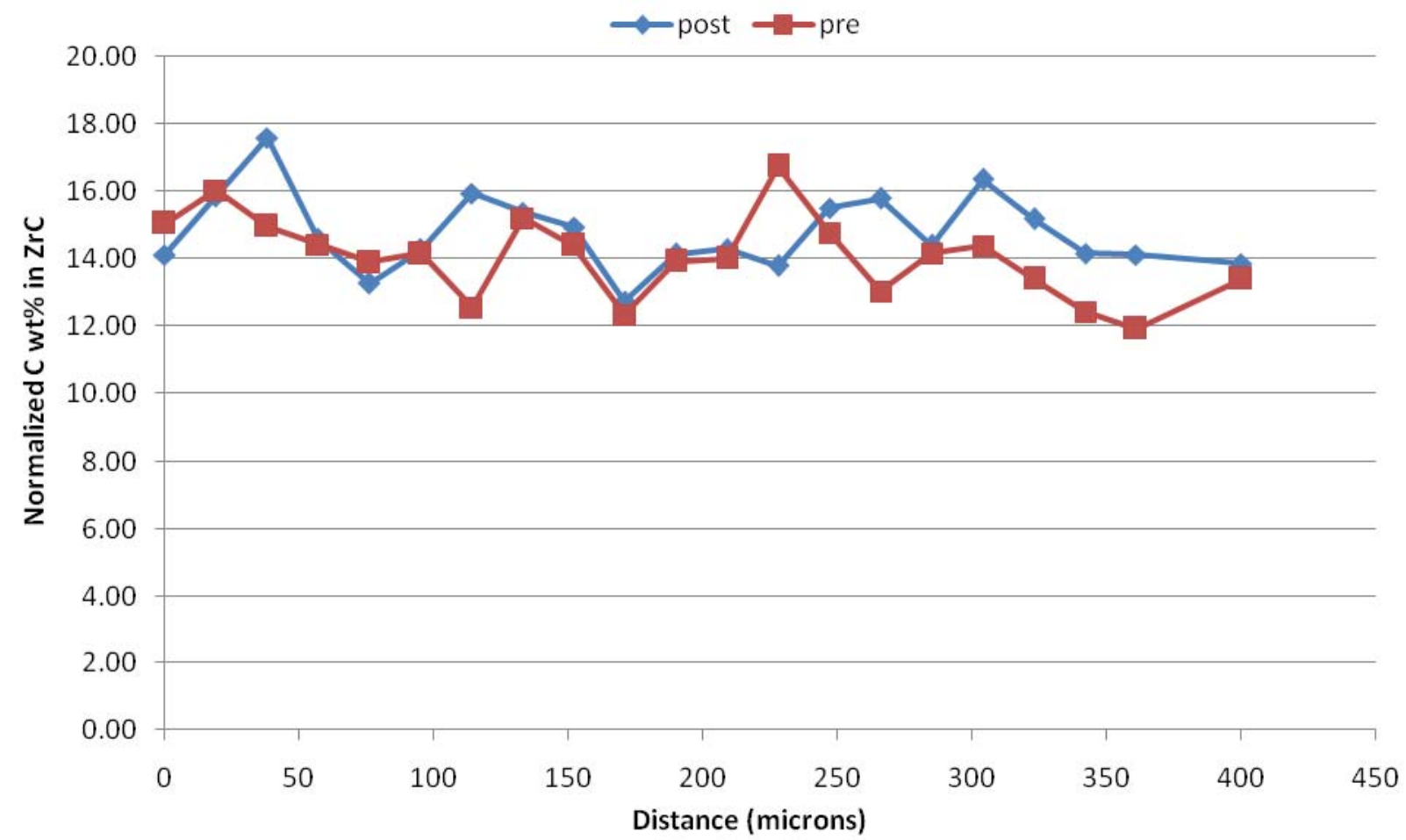

Figure 4-26. Mid-axial cross section showing $\mathrm{ZrC}$ carbon content as a function of depth. 


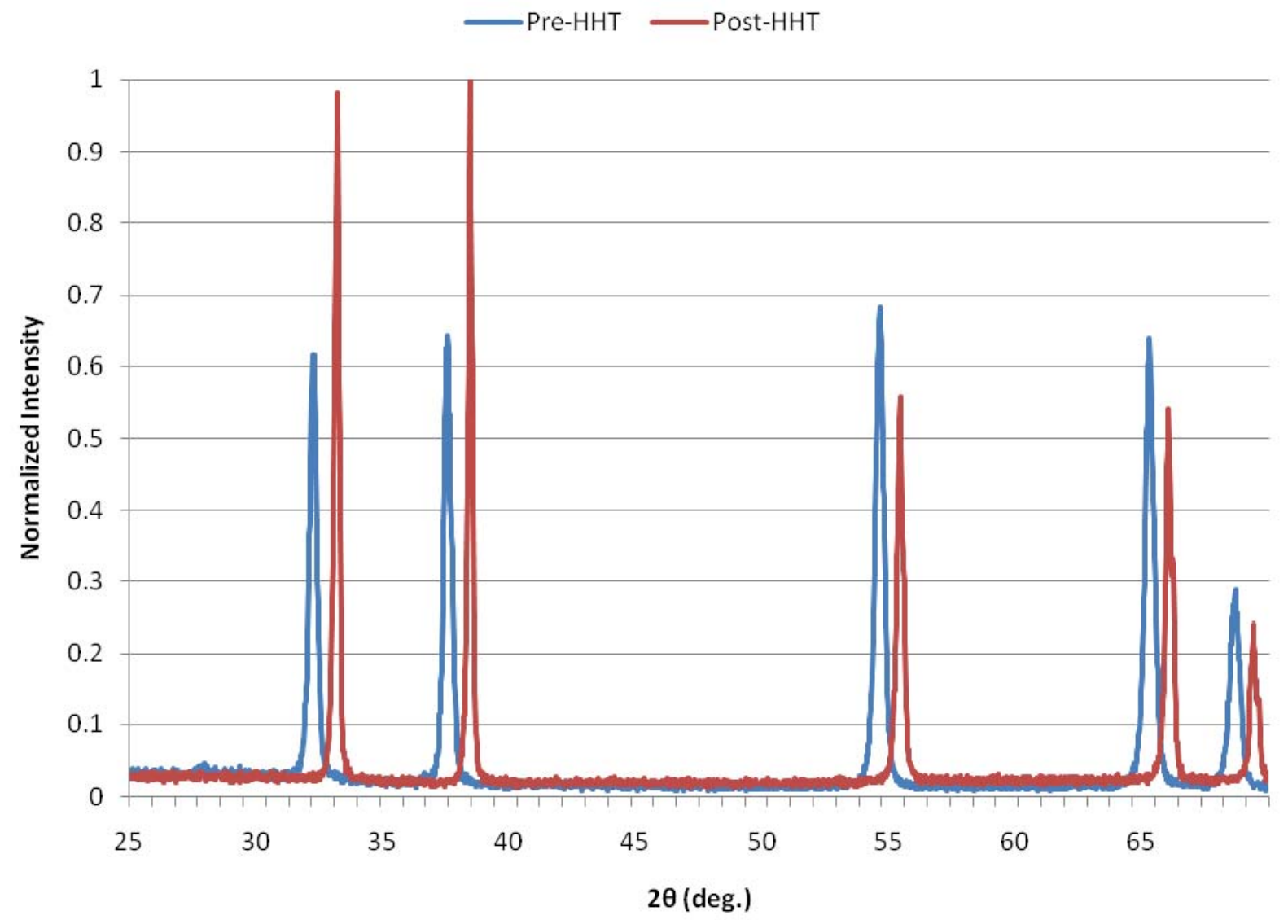

Figure 4-27. ZrC pre- and post-HHT XRD spectrum 

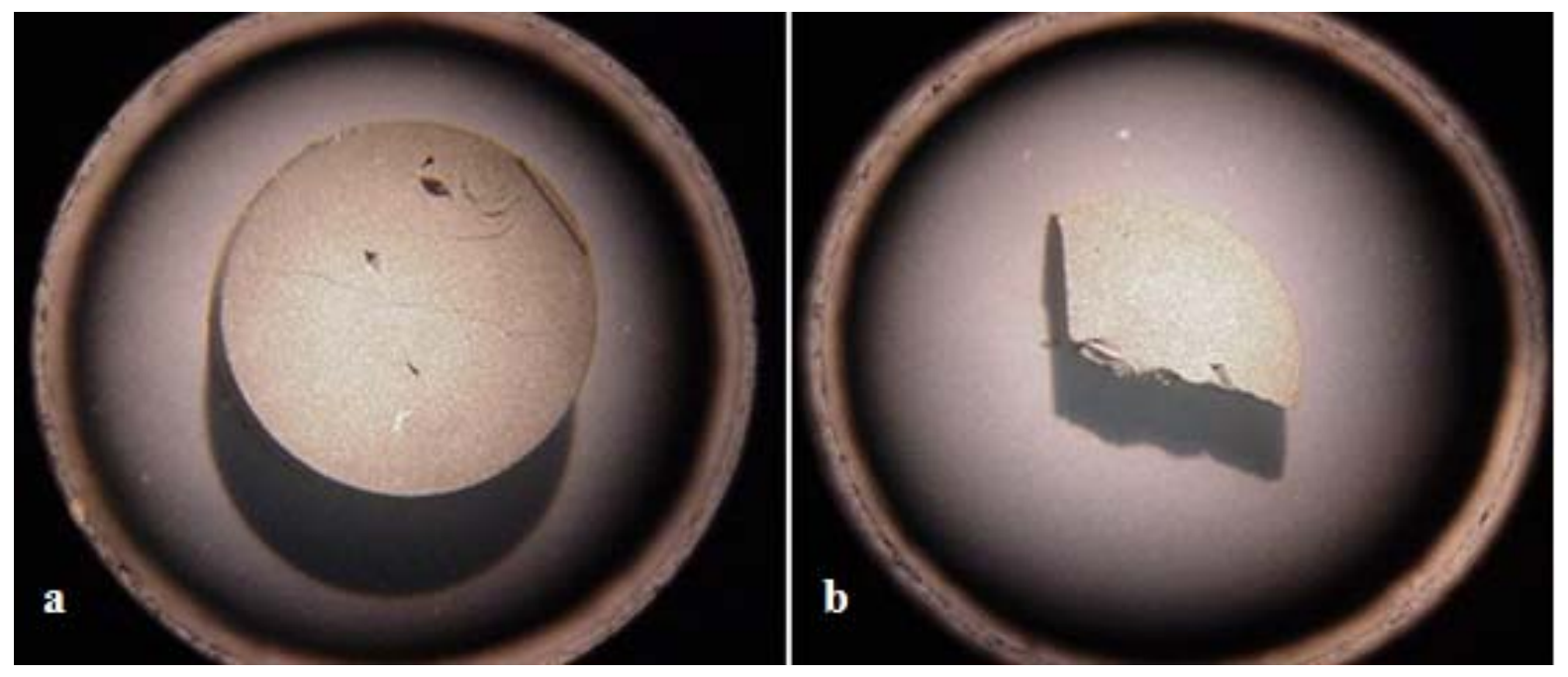

Figure 4-28. A) TRI-C1 pre-HHT. B) TRI-C1 post-HHT
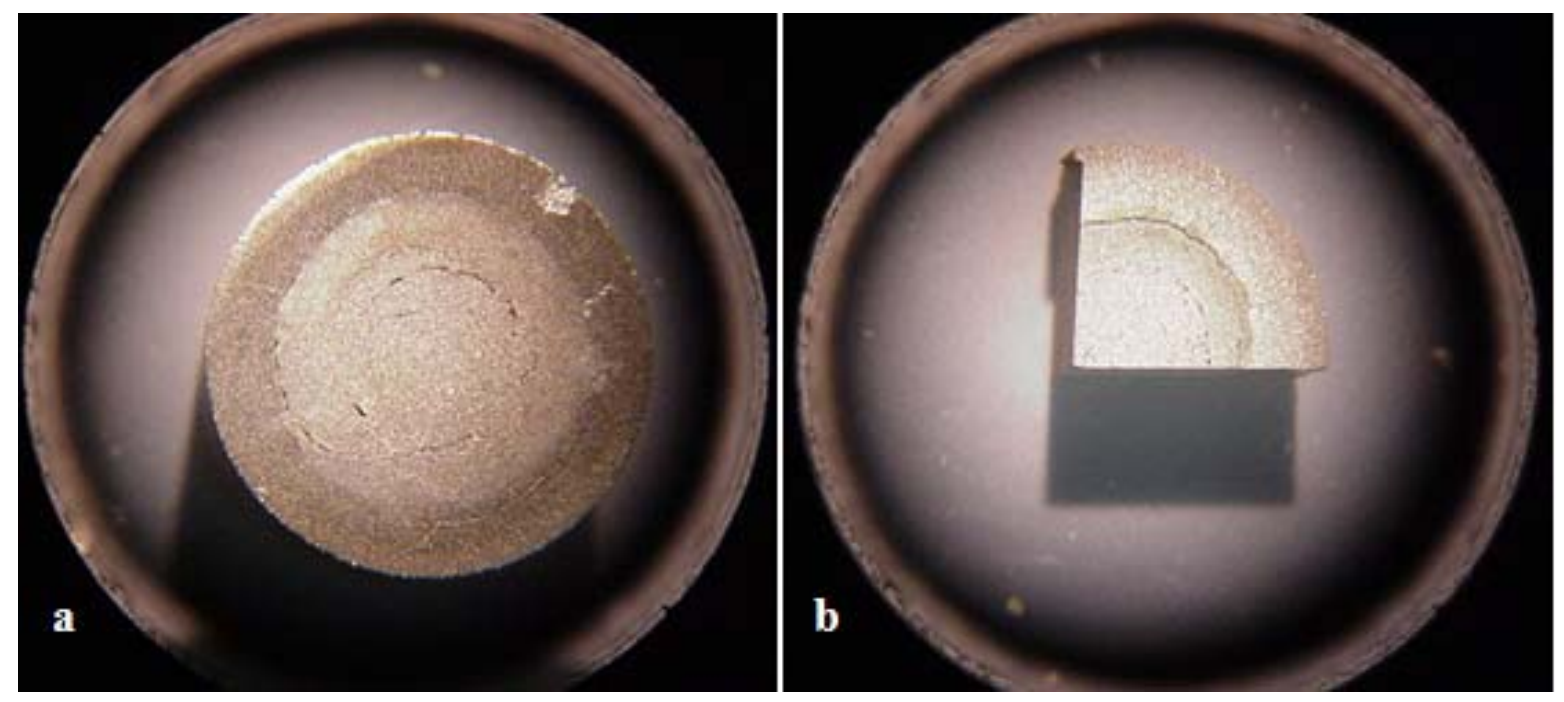

Figure 4-29. A) TRI-C2 pre-HHT. B) TRI-C2 post-HHT 


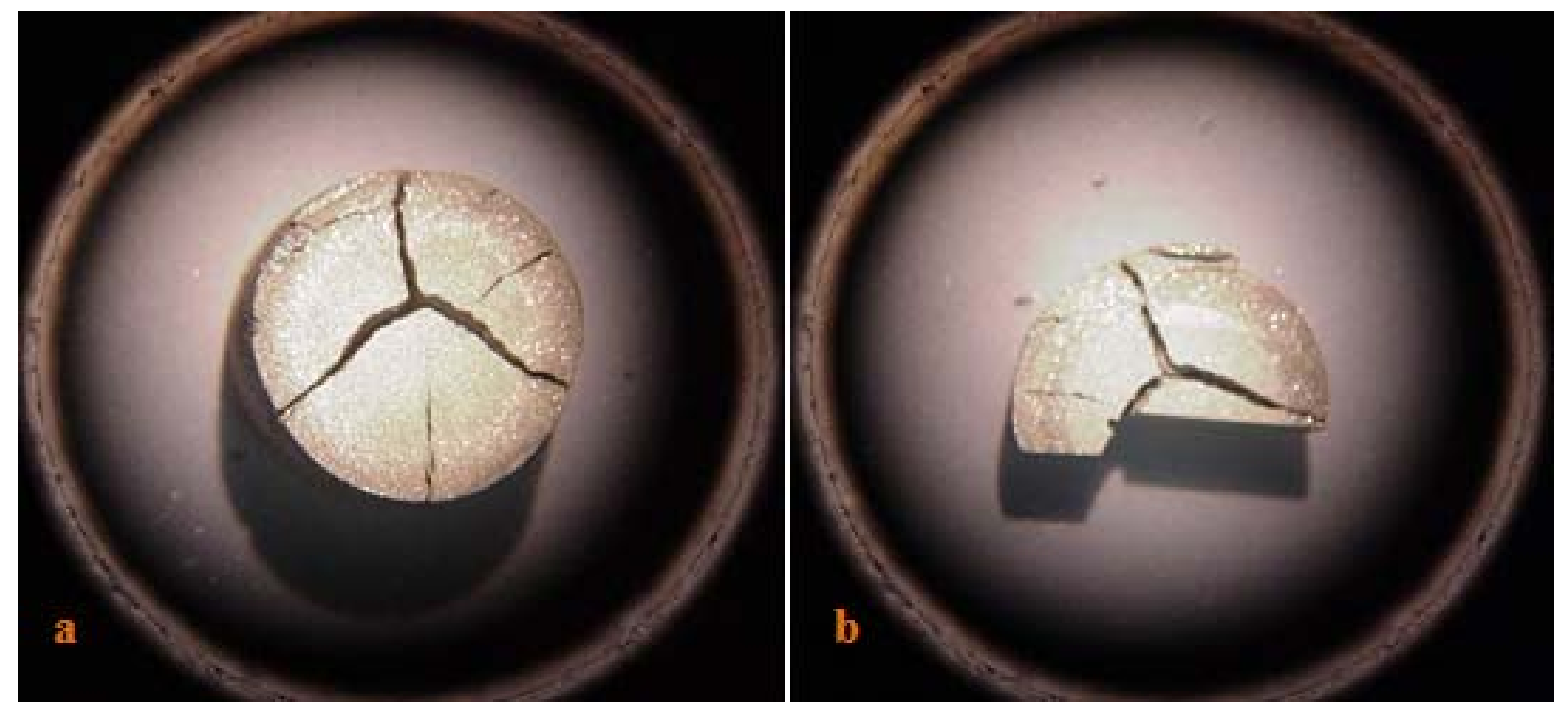

Figure 4-30. A) TRI-C3 pre-HHT. B) TRI-C3 post-HHT

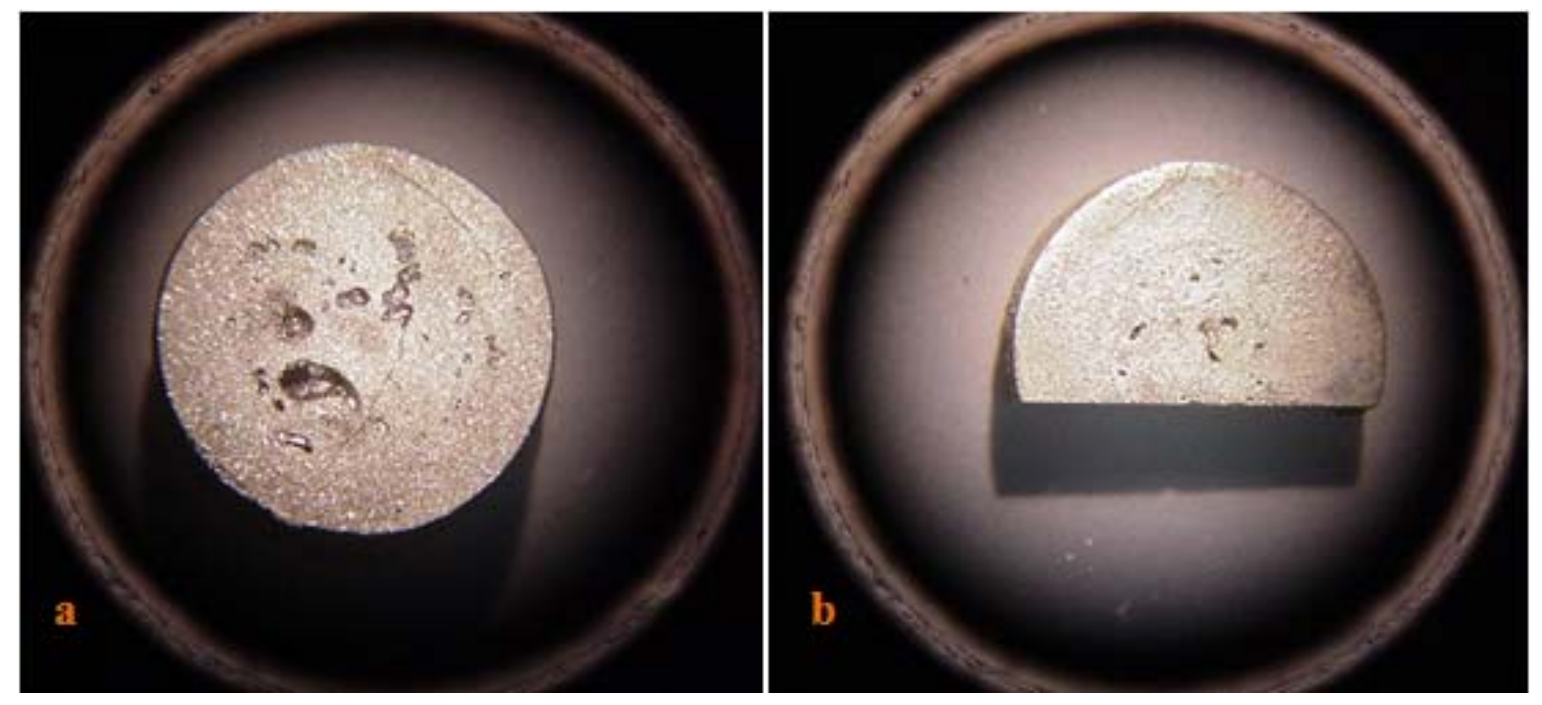

Figure 4-31. A) TRI-C4 pre-HHT. B) TRI-C4 post-HHT 


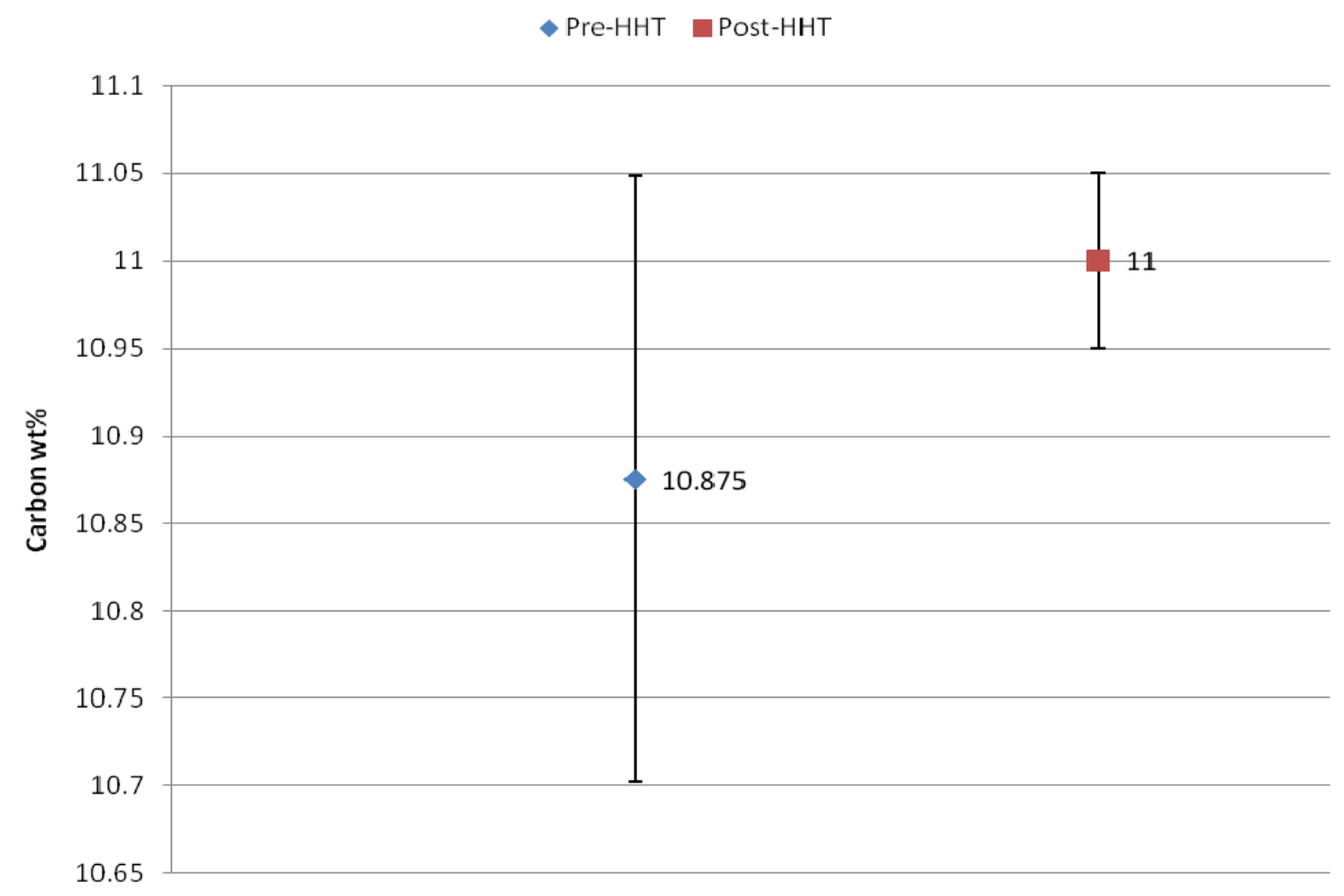

Figure 4-32. TRI-C1 pre- and post-HHT bulk carbon content

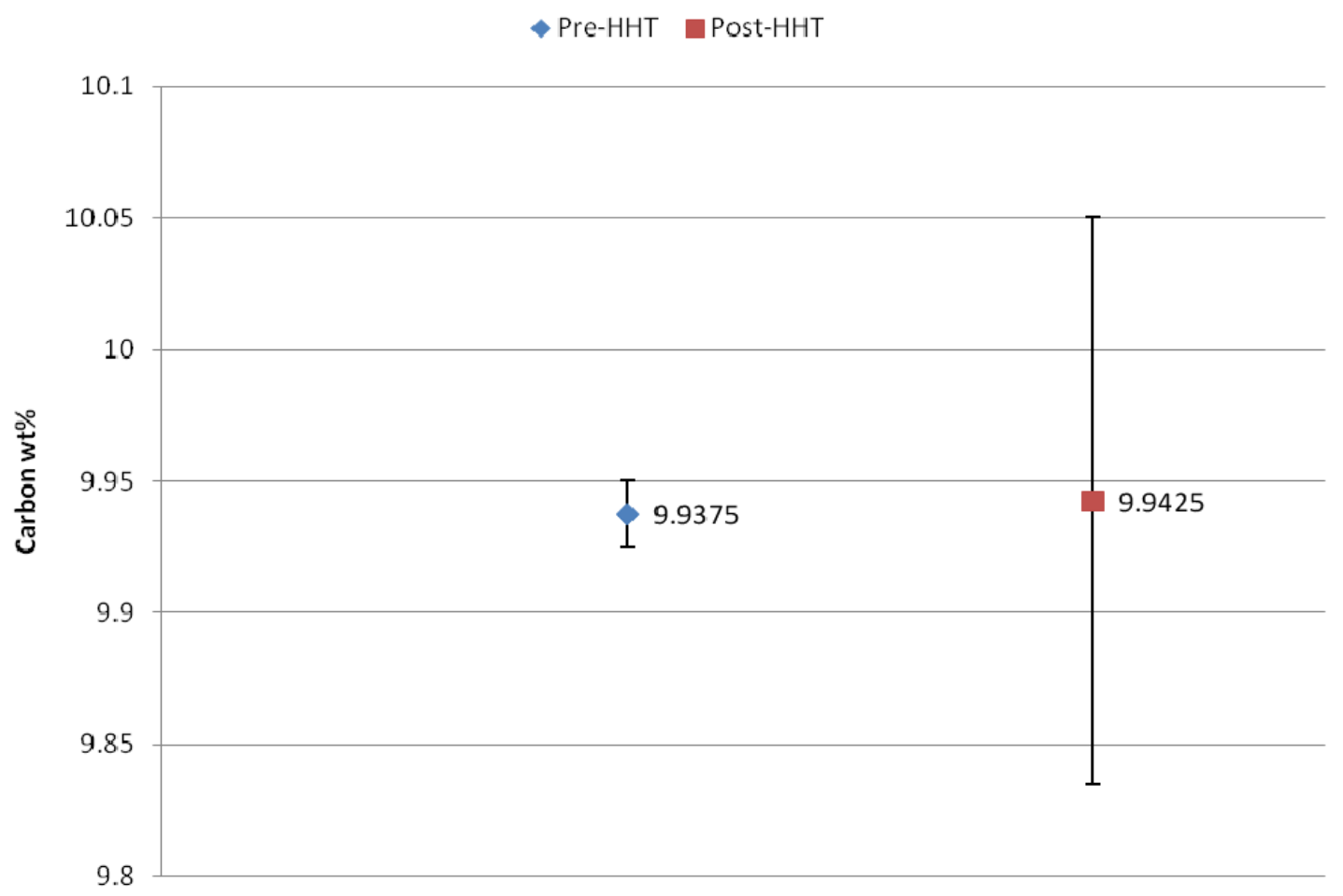

Figure 4-33. TRI-C2 pre- and post-HHT bulk carbon content 


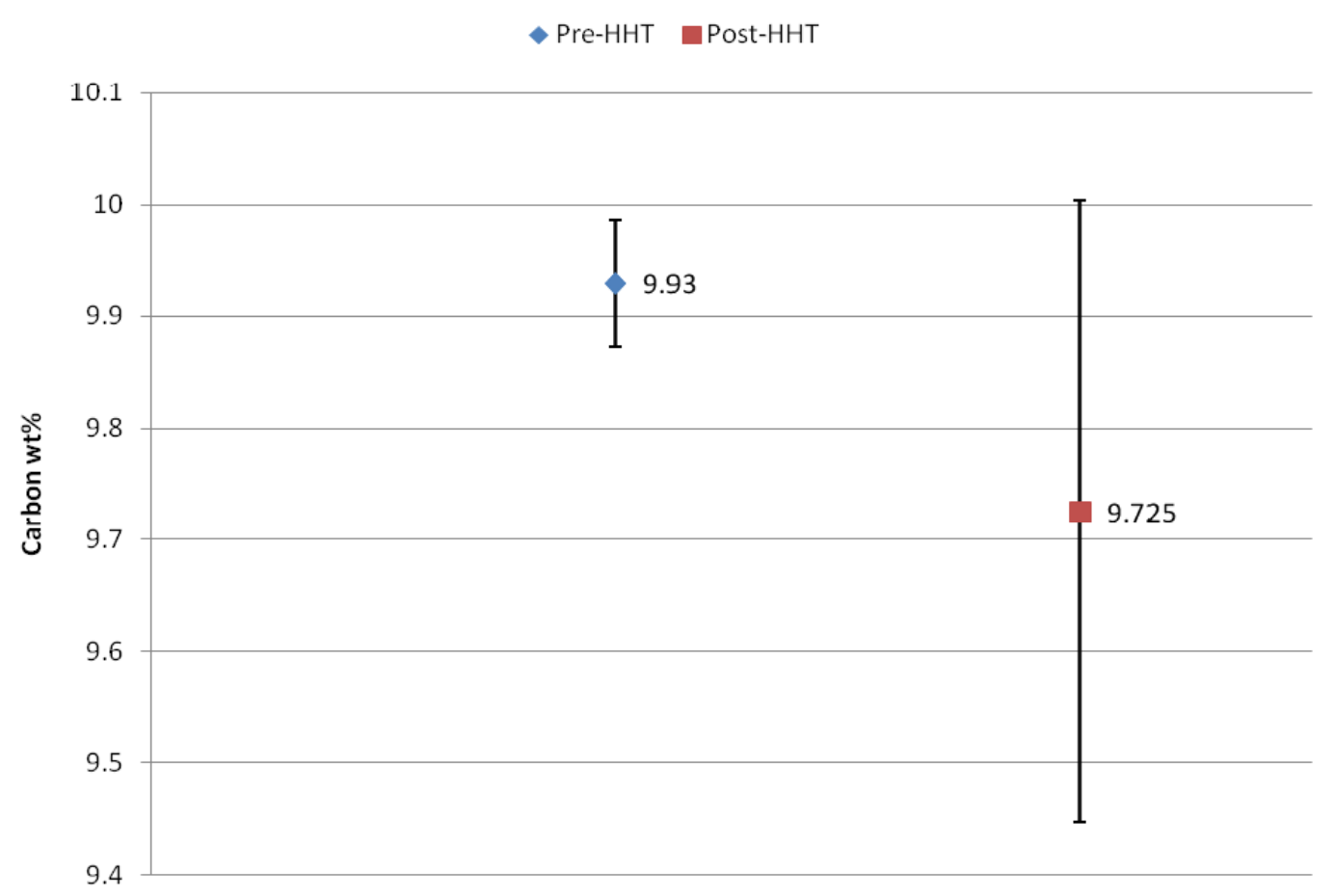

Figure 4-34. TRI-C3 pre- and post-HHT bulk carbon content

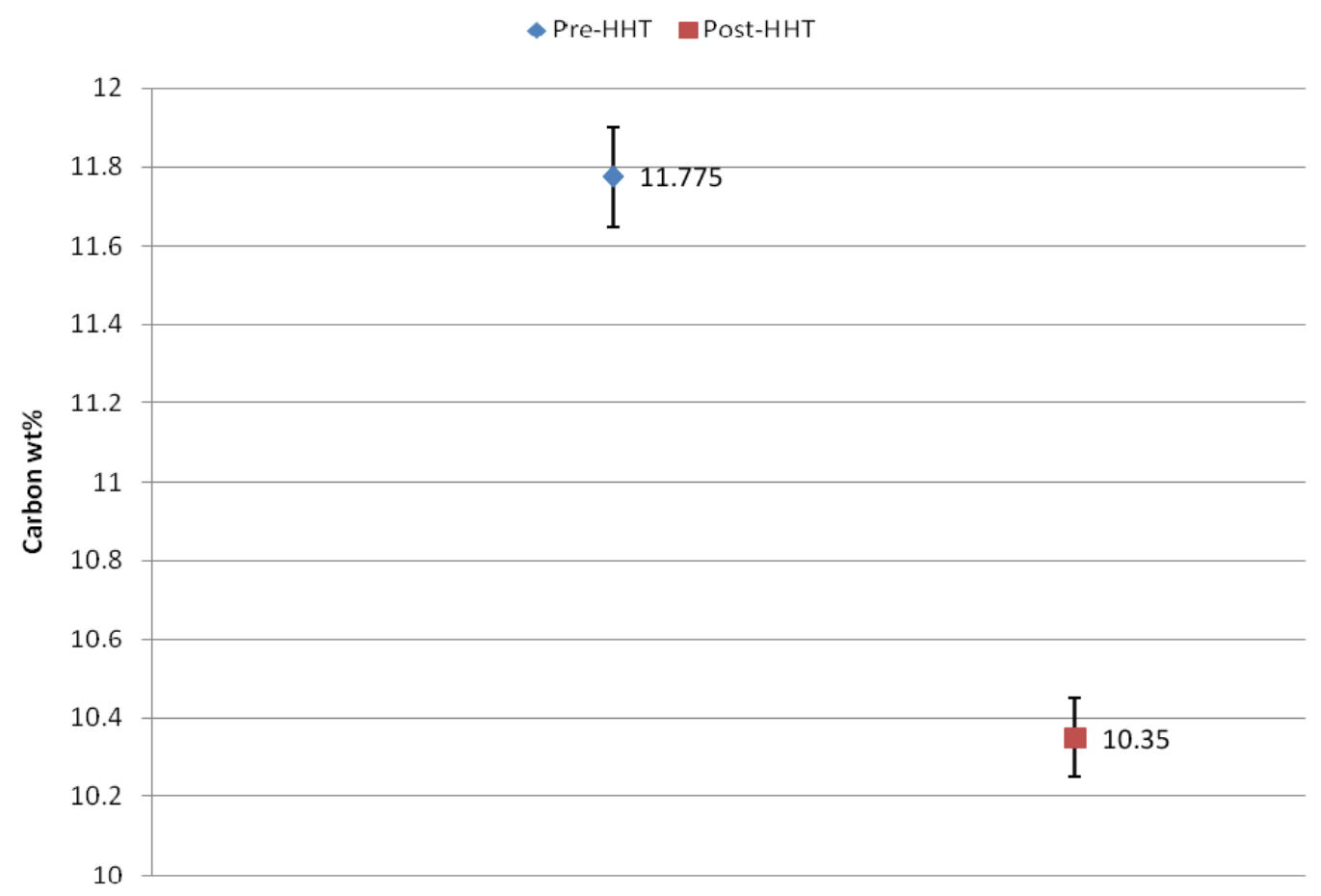

Figure 4-35. TRI-C4 pre- and post-HHT bulk carbon content 


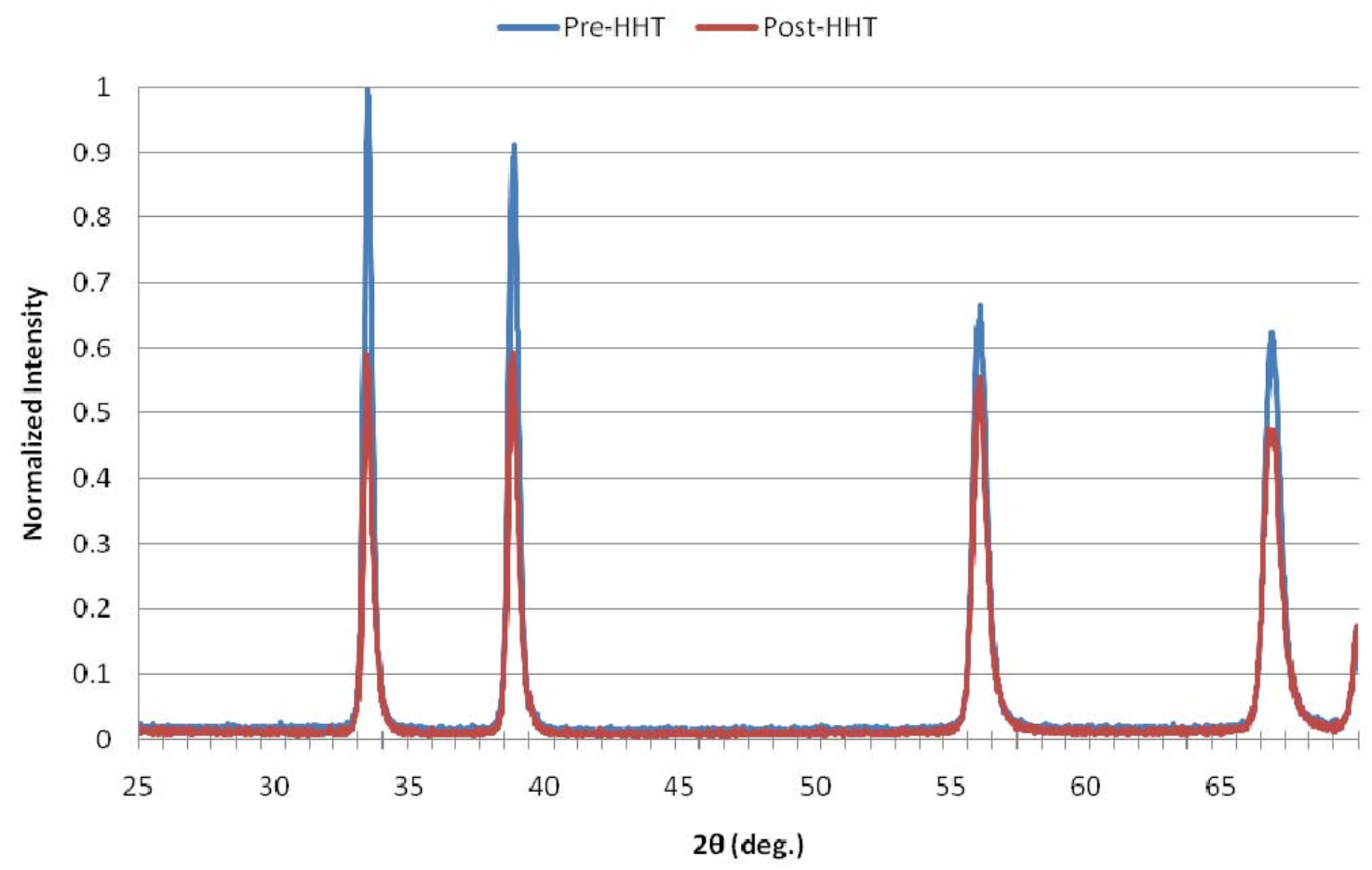

Figure 4-36. TRI-C1 XRD spectrum

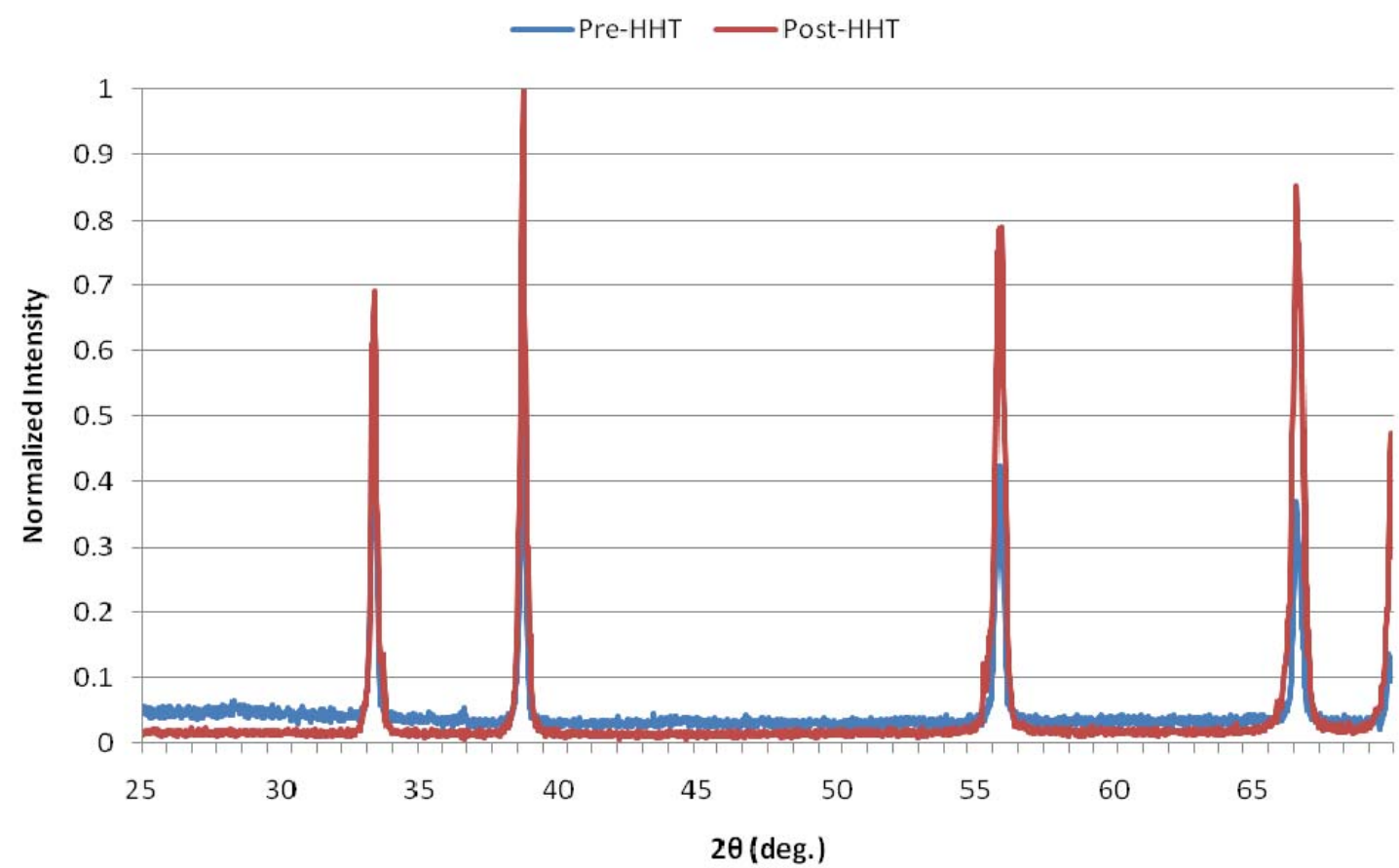

Figure 4-37. TRI-C2 XRD spectrum 


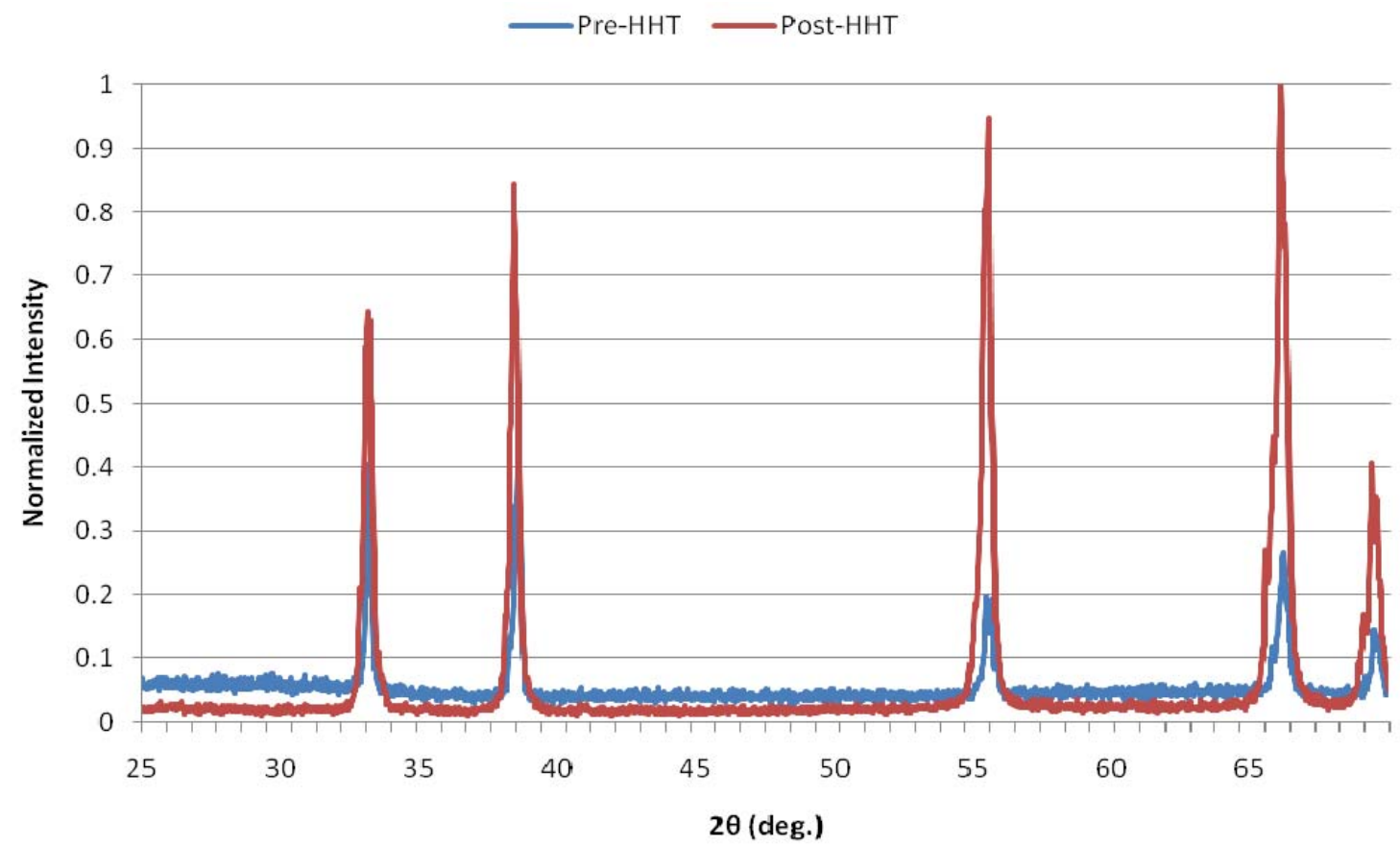

Figure 4-38. TRI-C3 XRD spectrum

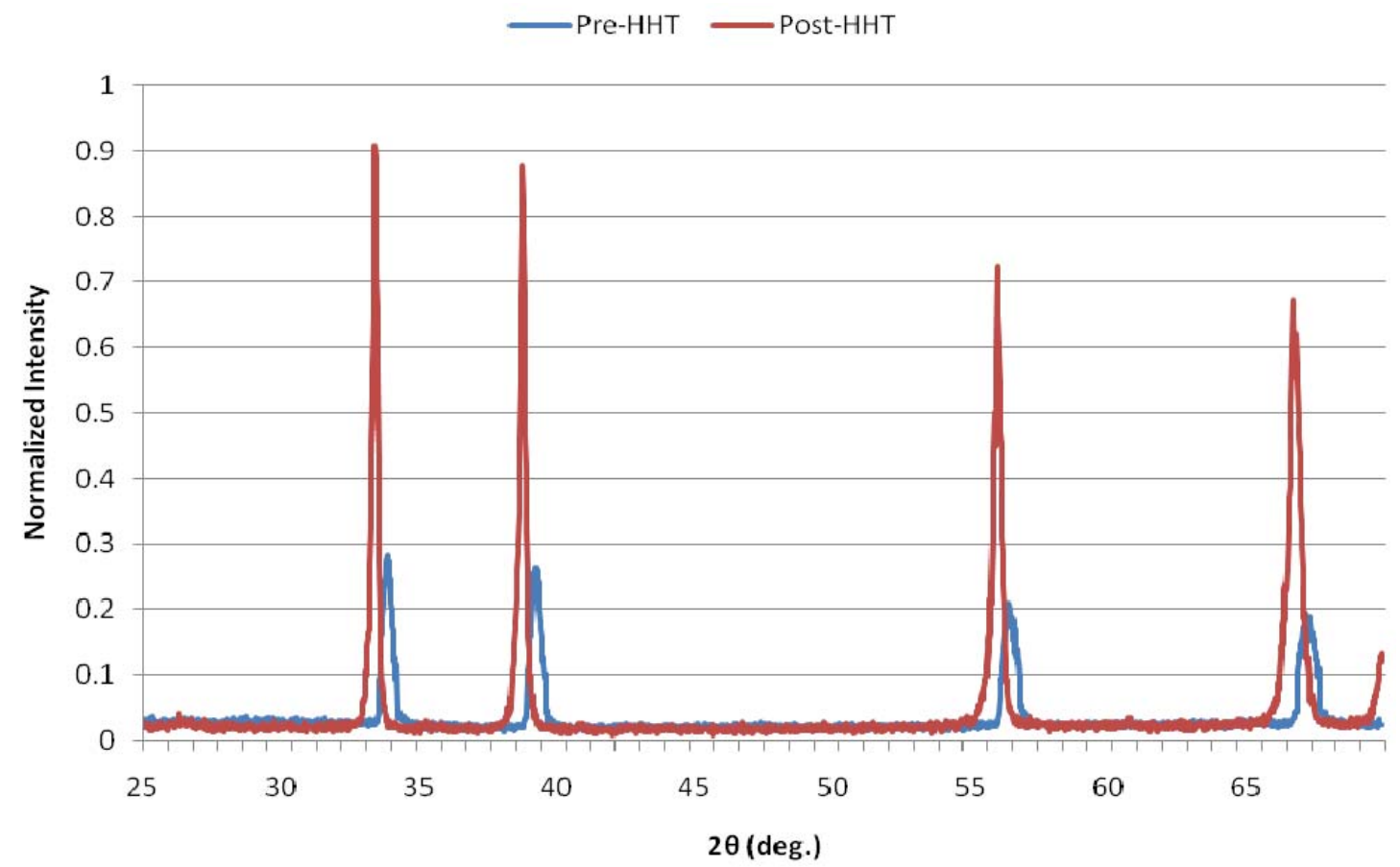

Figure 4-39. TRI-C4 XRD spectrum 


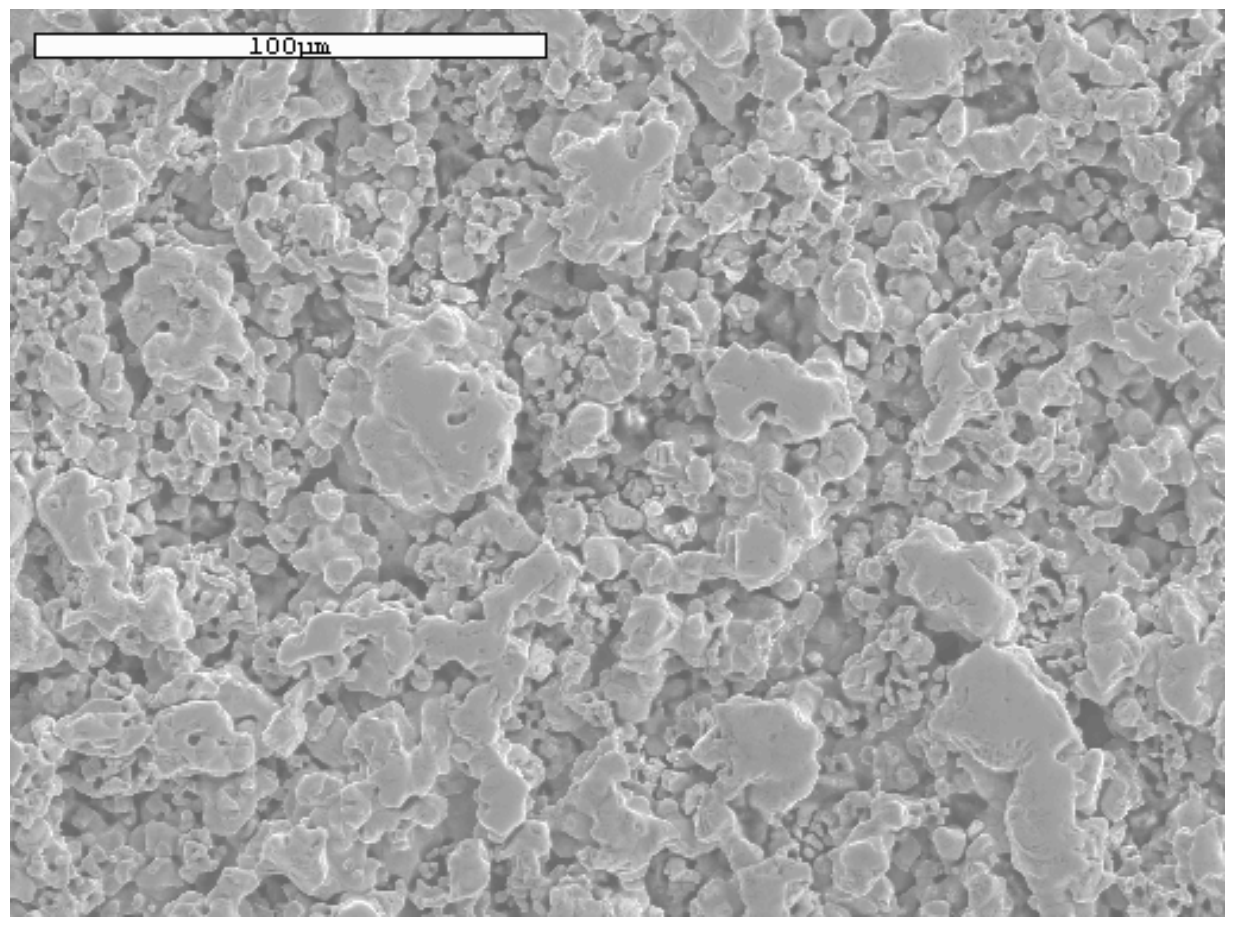

Figure 4-40. TRI-C1 SE image of pre-HHT specimen

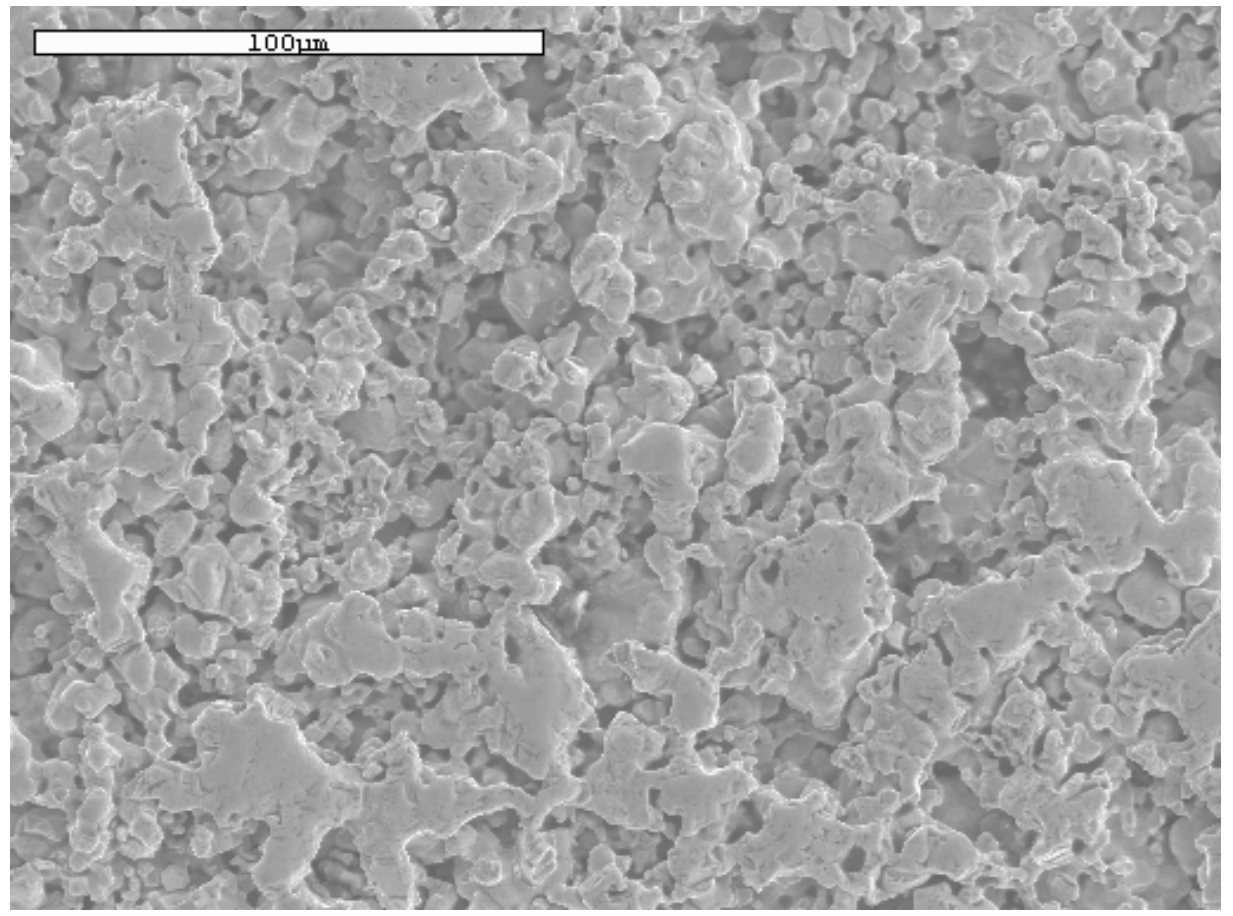

Figure 4-41. TRI-C1 SE image of post-HHT specimen 


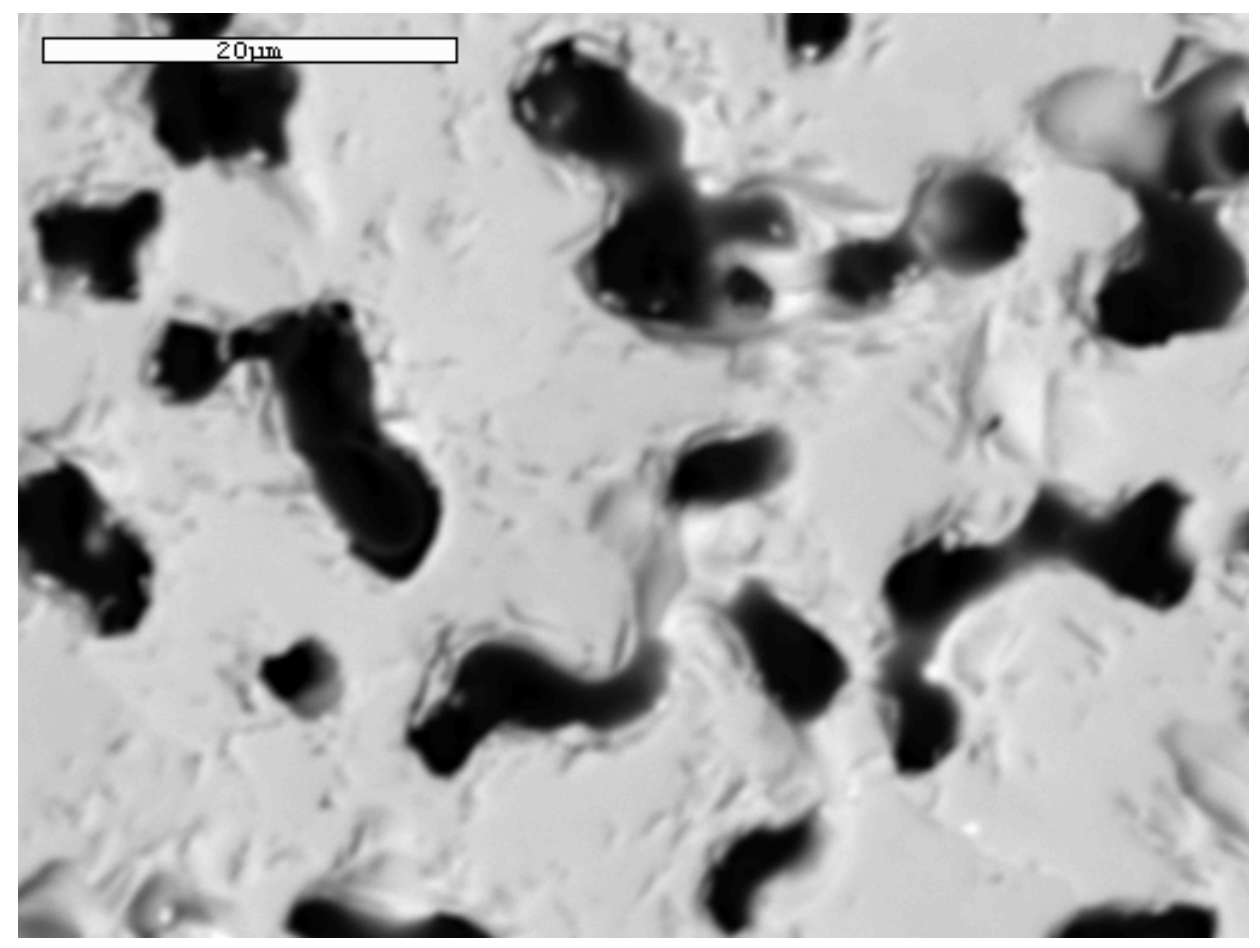

Figure 4-42. TRI-C2 BSE image of pre-HHT specimen

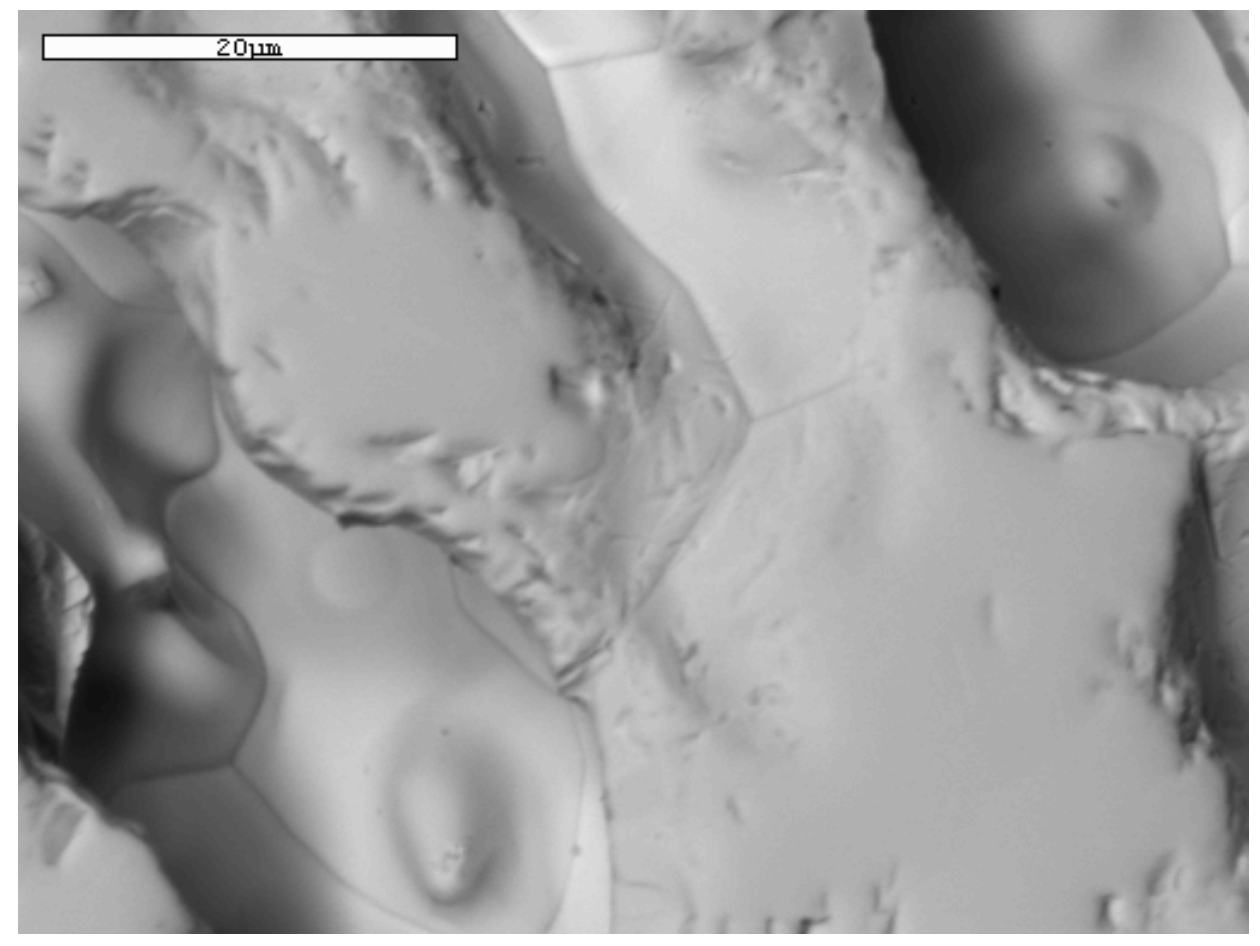

Figure 4-43. TRI-C2 BSE image of post-HHT specimen 


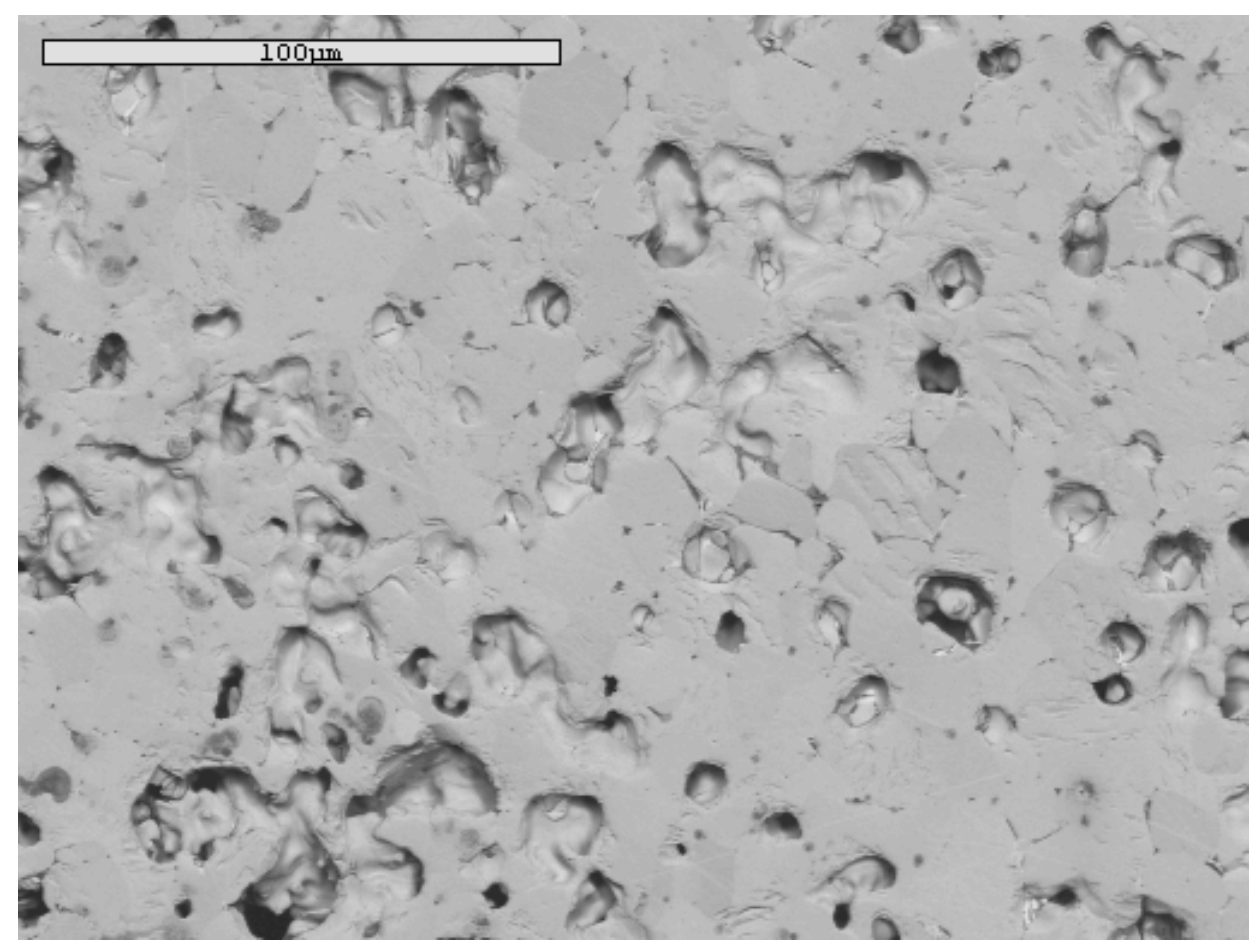

Figure 4-44. TRI-C3 BSE image of pre-HHT specimen

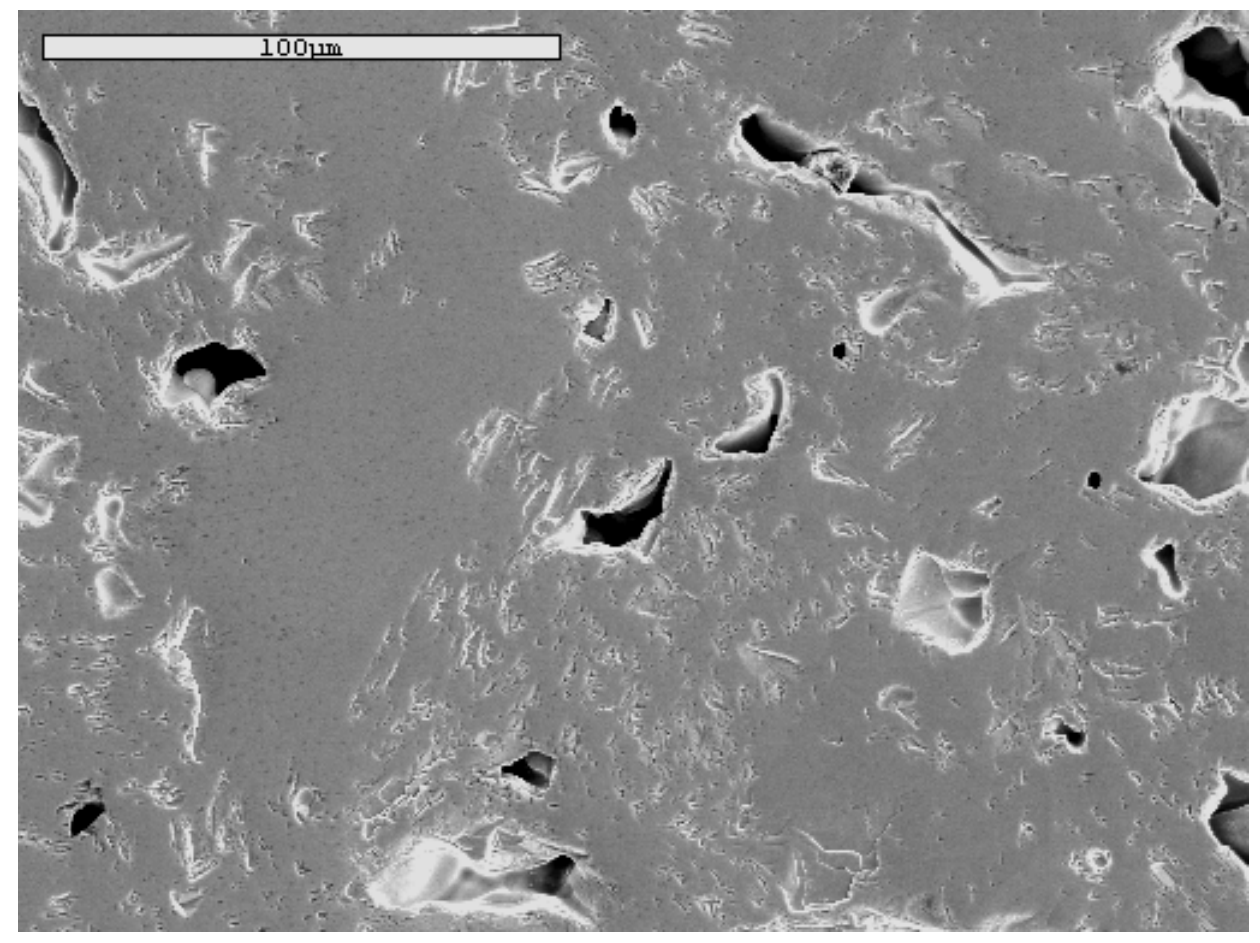

Figure 4-45. TRI-C3 SE image of post-HHT specimen 


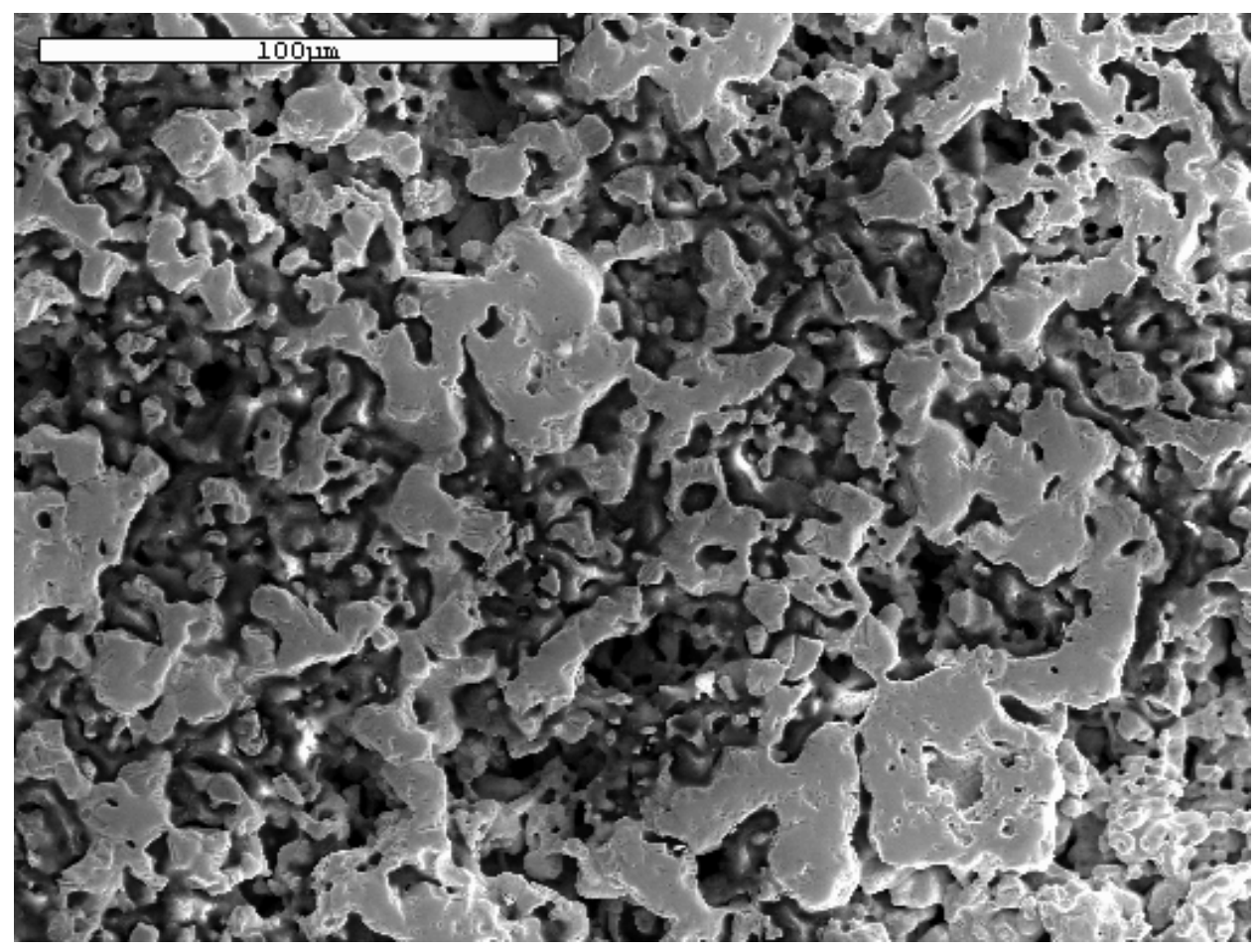

Figure 4-46. TRI-C4 SE image of pre-HHT specimen

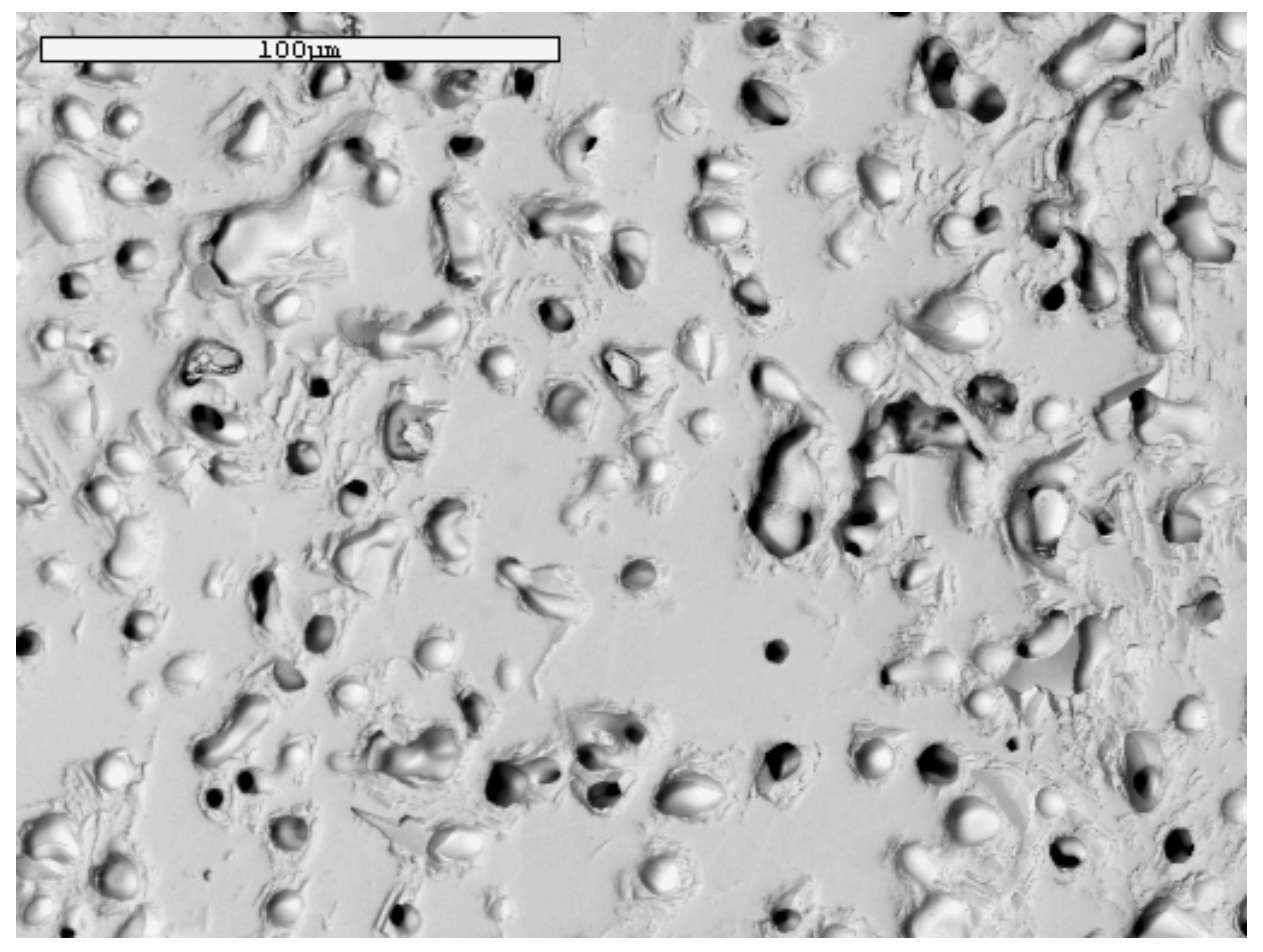

Figure 4-47. TRI-C4 BSE image of post-HHT specimen 


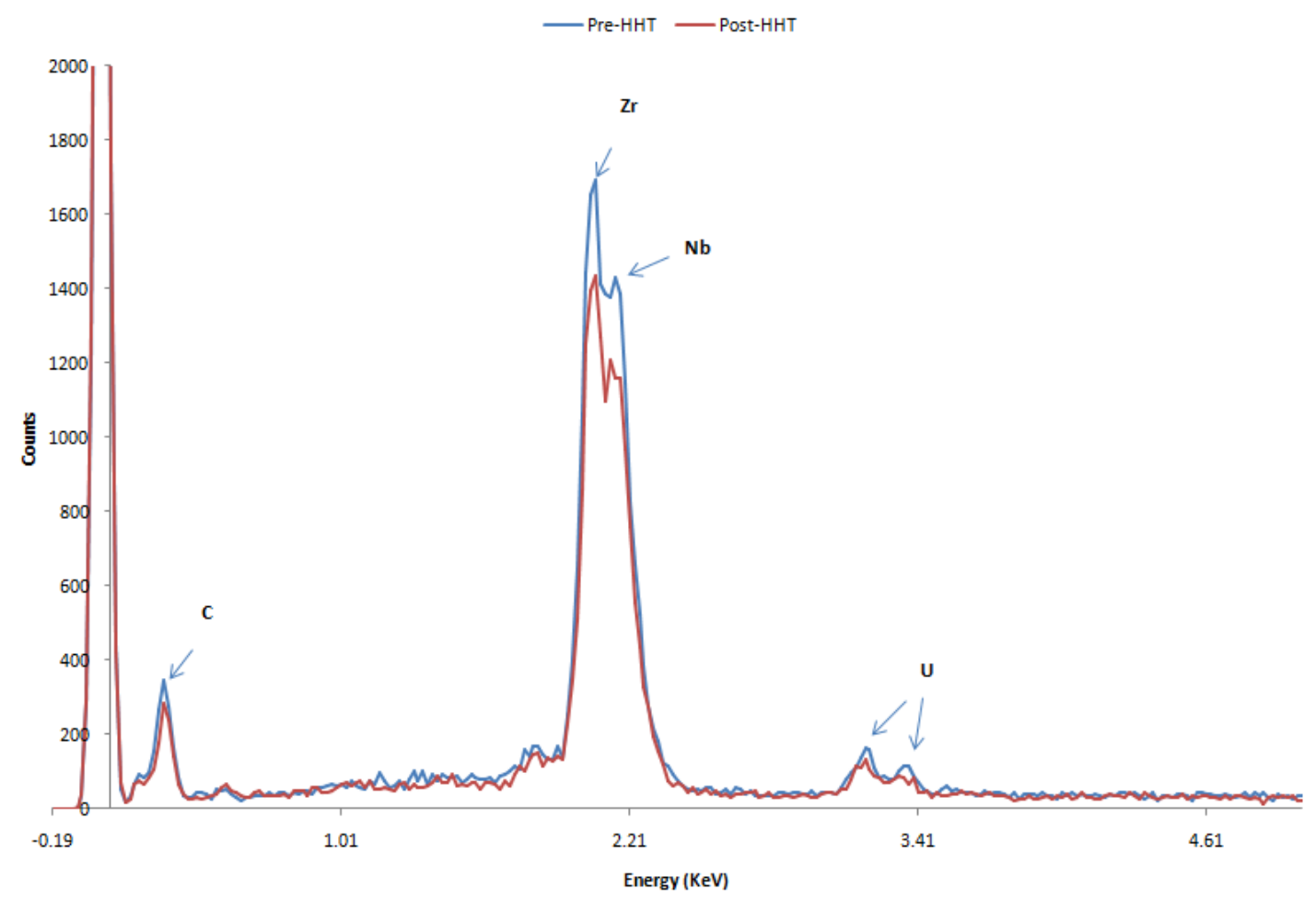

Figure 4-48. TRI-C1 pre- and post-HHT EDS

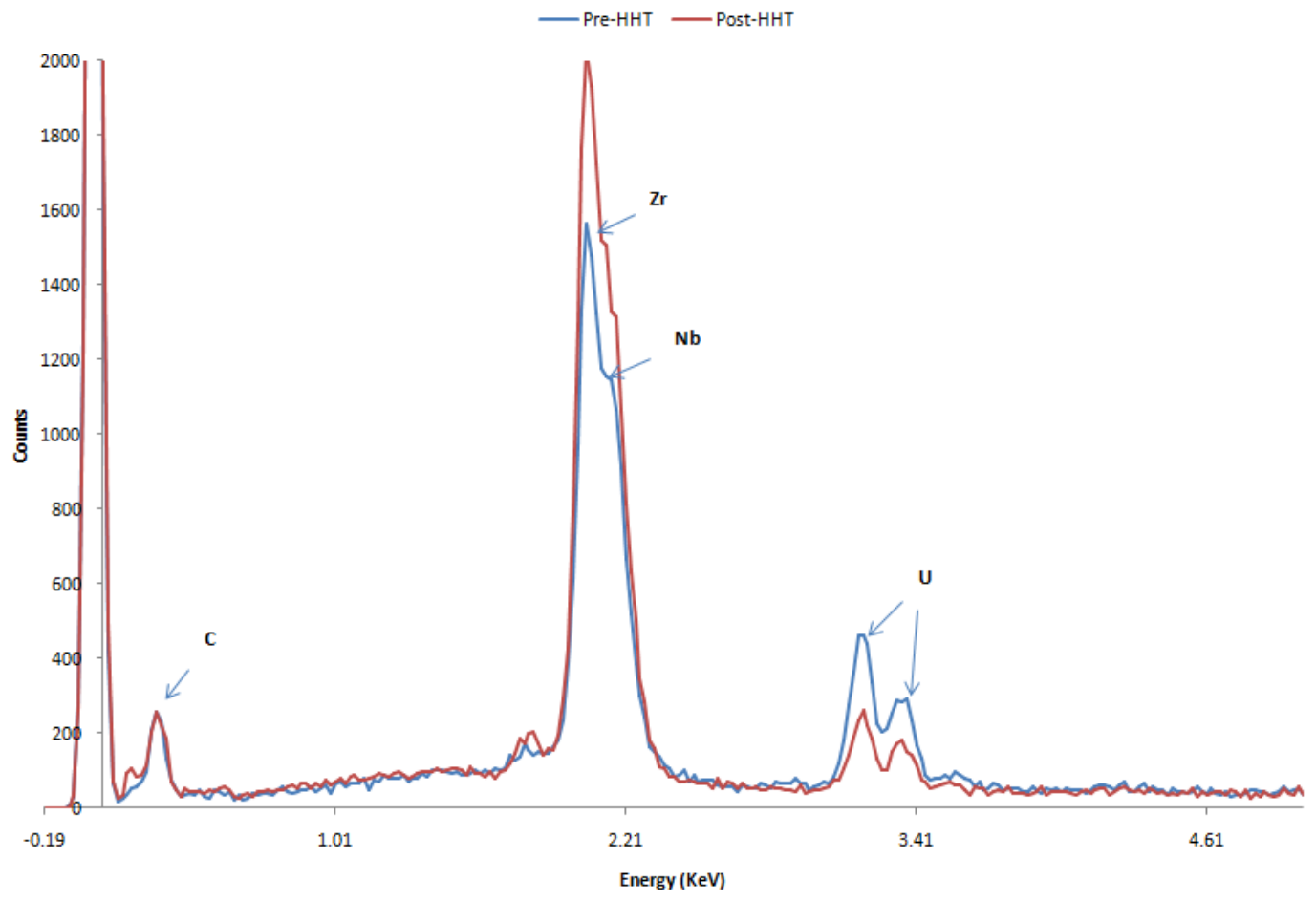

Figure 4-49. TRI-C2 pre-and post-HHT EDS. 


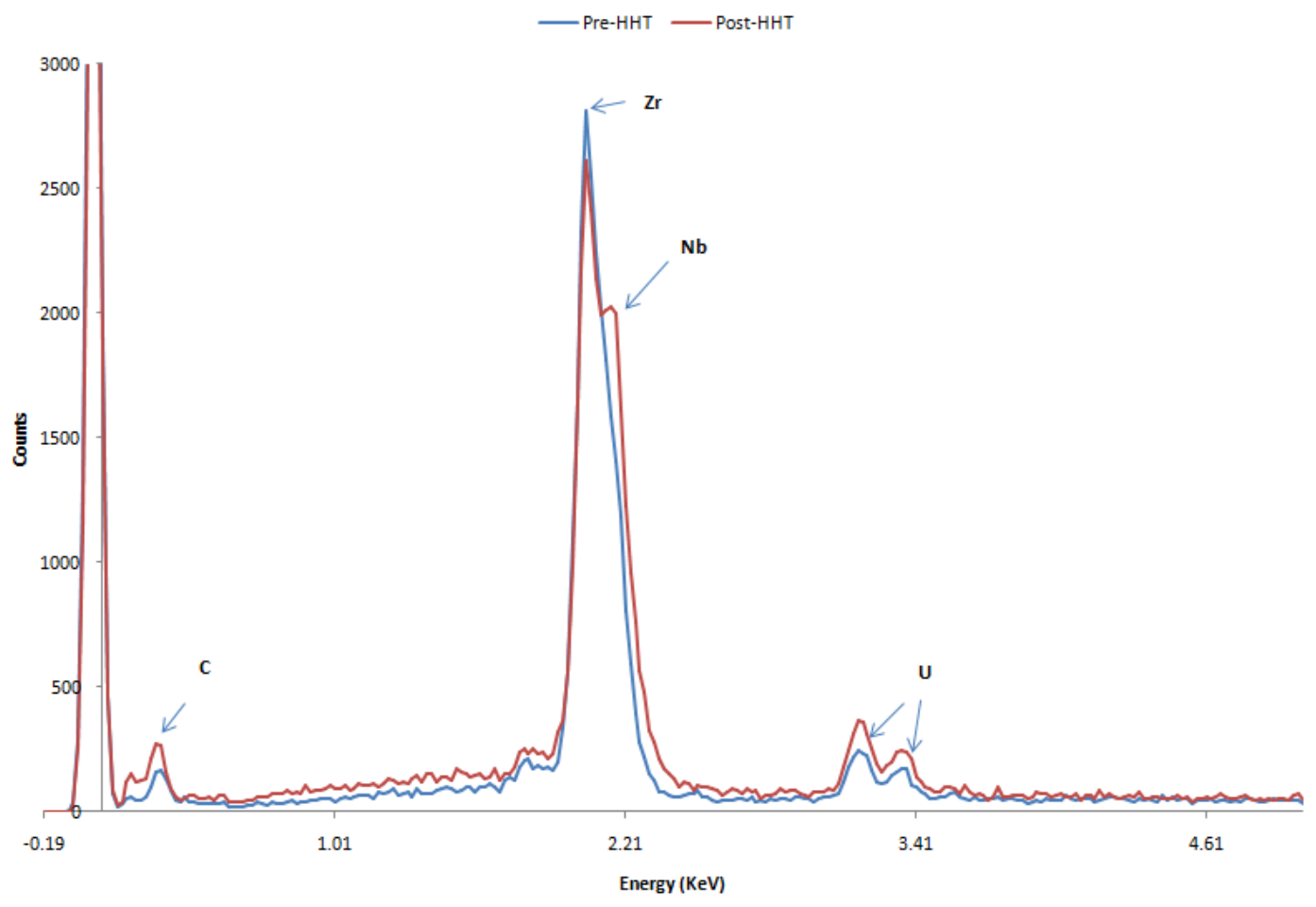

Figure 4-50. TRI-C3 pre-and post-HHT EDS.

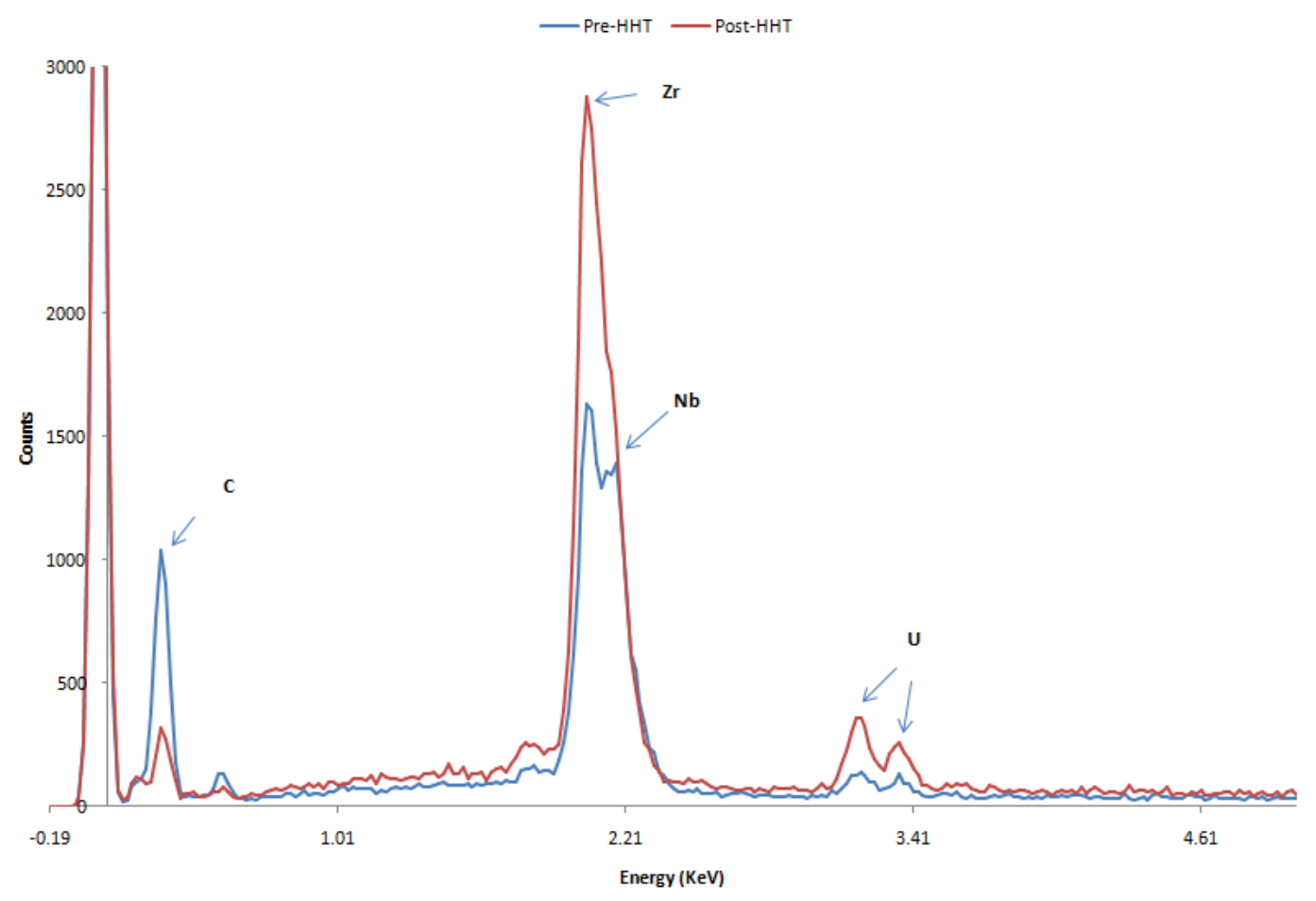

Figure 4-51. TRI-C4 pre-and post-HHT EDS 


\section{CONCLUSIONS AND RECOMMENDATIONS}

Testing the mono-carbides, $\mathrm{WC}, \mathrm{TaC}$, and $\mathrm{ZrC}$, in a hot hydrogen environment yielded differences that depended on the carbide composition. The sample that had the minimum change in microstructural integrity was the $\mathrm{ZrC}$ sample. The sample's carbon content remained the same. No cracking, grain growth, density changes, or hydrogen embrittlement was observed. The XRD spectrum showed the post-HHT specimen to have no change in phase from the preHHT specimen.

The TaC post-HHT sample performed the second best in having minimum degradation on microstructural integrity. There was no depletion of carbon from the sample, both bulk and cross sectional; however, the sample did experience extensive cracking and micro-pores surrounding the fractures. A density decrease of $4.836 \%$ in the post-HHT sample was measured against the pre-HHT sample.

The WC sample performed the worst in a hot hydrogen environment. The post-HHT sample experienced a noticeable degree of phase change, grain boundary separation, carbon depletion, and possible formation hydrocarbons. Portions of the post-HHT sample's XRD spectrum showed the formation of $\mathrm{W}_{2} \mathrm{C} . \mathrm{W}_{2} \mathrm{C}$ most likely formed on the edge where carbon depletion occurred. After a two hour test at $2775 \mathrm{~K}$, the hydrogen was shown to deplete carbon

from the WC matrix at rate of $5.25 \mathrm{\mu m} / \mathrm{min}$. WC also experienced the greatest change in density, decreasing by $10.91 \%$. Based on these results WC would not be a safe fuel candidate for NTP because of the threat for fuel failure due to changes in the microstructure.

Testing of the four solid solution uranium tri-carbides was also conducted for the representative two hour NTP operating time but at higher temperatures, approximately $2900 \mathrm{~K}$. Analysis showed the tri-carbides displayed little to no evidence of changes due to the hot 
hydrogen environment. The bulk carbon analysis showed that three of the four carbides displayed constant carbon content from pre- to post-HHT status. The fourth tri-carbide sample reported a lost in carbon content. This was most likely due to carbon contamination during processing and handling of the specimen. The SEM images showed no regions of microstructural segregation with particular attention given to the surface since the surface is where the depletion of carbon would be the greatest. The XRD patterns verified the SEM analysis and bulk carbon analysis by showing that the tri-carbide specimens remained as unaffected solid solution carbides. Based on the analysis, solid solution uranium tri-carbides appear to be compatible with hydrogen to a maximum tested temperature of $2926 \mathrm{~K}$ for two hours.

Hydrogen corrosion due to temperature is only one mechanism that can affect the rate at which hydrogen can attack a fuel matrix. Future work in investigating carbides and more importantly tri-carbides as NTP fuel should look to examine the effects of other corrosion mechanism such as fuel irradiation. Since the hot hydrogen corrosion process consist of temperature effects coupled with radiation exposure and thermal mechanical stresses, future test should attempt to incorporate multiple corrosion mechanisms. 


\section{LIST OF REFERENCES}

[1]. Knight, T. W., "Processing of Solid Solution Mixed Uranium/Refractor Metal Carbides for Advanced Space Nuclear Power and Propulsion Systems" Ph.D. Dissertation, University of Florida, 2000.

[2]. Anghaie, S., Knight, T. W., "Development of Robust Tri-carbide Fueled Reactors for Multi-Megawatt Space Power and Propulsion Applications”, American Nuclear Society 2004 Winter Meeting, 2004.

[3]. Butt, D. P., Pelaccio, D. G., El-Genk, M. S., "A Review of Carbide Fuel Corrosion for Nuclear Thermal Propulsion Applications", $11^{\text {th }}$ Symposium of Space Nuclear Power and Propulsion Conference, January, 1994.

[4]. Pewee Reactor Test Report, Los Alamos National Laboratory August 1969.

[5]. Koenig, D. R., "Experience Gained from the Space Nuclear Rocket Program (Rover)", Los Alamos National Laboratory, May, 1986.

[6]. Pelaccio, D. G., El-Genk, M. S., Butt, D. P., "Hydrogen Corrosion Consideration of Carbide Fuel for Nuclear Thermal Propulsion Applications", Journal of Propulsion and Power, Vol. 11 No6, November 1995.

[7]. Sandlers, S. I., Chemical and Engineering Thermodynamics $3^{\text {rd }}$ Ed., New York, John Wiley \& Sons, Inc., 1999.

[8]. NIST-JANAF Thermochemical Tables, $3^{\mathrm{RD}}$ Ed., 1985.

[9]. NIST-JANAF Thermochemical Tables, $4^{\mathrm{TH}}$ Ed., 1998.

[10]. Seng, W. F., Barnes, P. A., "Calculations of Tungsten Silicide and Carbide Formation on $\mathrm{SiC}$ using the Gibbs Free Energy" Material Science and Engineering B, Elsevier, Vol. 72 Issue 1, March 2000.

[11]. JCPDS International Centre for X-Ray Diffraction Data. Standard X-Ray Diffraction Powder Patterns from the JCPDS Research Associateship. s.l. : JCPDS International Centre for X-Ray Diffraction Data, 1996.

[12]. Boeing Aerospace Company, "Advanced Propulsion Systems Concepts for Orbital Transfer Study”, NASA MSFC, 1981

[13]. Knight, T. W., Anghaie, S., "Processing and Fabrication of Mixed Uranium/Refractory Metal Carbide Fuels with Liquid-Phase Sintering”, Journal of Nuclear Materials, Elsevier, Vol. 306, 2002

[14]. Panda, B., Hickman, R. R., Sandeep, S., Solid Solution Carbides are the Key Fuels for Future Nucelar Thermal Propulsion”, NASA MSFC, 2005 
[15]. Porter, D. A., Easterling, K. E., Phase Transformation in Metals and Alloys $2^{\text {nd }}$ Ed., Florida, CRC Press, 1992.

[16]. Wang, C. R., Yang, J. M., Hoffman, W., "Thermal Stability of Refractory Carbide/boride Composites", Materials, Chemistry and Physics, Elsevier, Vol. 72 Issue 3, March 2002.

[17]. Zee, R., Chin, B., Cohron, J., "Hot Hydrogen Testing of Refractory Metals and Ceramics" Auburn Univ., AL, February 1993. 
